\title{
TOUGH+HYDRATE v1.0
}

USER'S MANUAL:

A Code For THE Simulation OF SYSTEM BEHAVIOR IN HYDRATEBeARing Geologic Media

\section{George J. Moridis, Michael B. Kowalsky, and Karsten Pruess}

Earth Sciences Division, Lawrence Berkeley National Laboratory, Berkeley, CA 94720

\section{March 2008}

This work was supported by the Assistant Secretary for Fossil Energy, Office of Natural Gas and Petroleum Technology, through the National Energy Technology Laboratory, under the U.S. Department of Energy, Contracts No. DE-AC03-76SF00098 and DE-AC02-05CH11231. 


\title{
TOUGH+HYDRATE v1.0 User's Manual: A Code for the Simulation of System Behavior in Hydrate-Bearing Geologic Media
}

\author{
George J. Moridis, Michael B. Kowalsky, and Karsten Pruess \\ Earth Sciences Division, Lawrence Berkeley National Laboratory \\ University of California, Berkeley, California
}

\begin{abstract}
TOUGH+HYDRATE v1.0 is a new code for the simulation of the behavior of hydrate-bearing geologic systems. By solving the coupled equations of mass and heat balance, TOUGH+HYDRATE can model the non-isothermal gas release, phase behavior and flow of fluids and heat under conditions typical of common natural $\mathrm{CH}_{4}$-hydrate deposits (i.e., in the permafrost and in deep ocean sediments) in complex geological media at any scale (from laboratory to reservoir) at which Darcy's law is valid.

TOUGH+HYDRATE v1.0 includes both an equilibrium and a kinetic model of hydrate formation and dissociation. The model accounts for heat and up to four mass components, i.e., water, $\mathrm{CH}_{4}$, hydrate, and water-soluble inhibitors such as salts or alcohols. These are partitioned among four possible phases (gas phase, liquid phase, ice phase and hydrate phase). Hydrate dissociation or formation, phase changes and the corresponding thermal effects are fully described, as are the effects of inhibitors. The model can describe all possible hydrate dissociation mechanisms, i.e., depressurization, thermal stimulation, salting-out effects and inhibitor-induced effects.

TOUGH+HYDRATE is the first member of TOUGH+, the successor to the TOUGH2 [Pruess et al., 1991] family of codes for multi-component, multiphase fluid and heat flow developed at the Lawrence Berkeley National Laboratory. It is written in standard FORTRAN 95, and can be run on any computational platform (workstation, PC, Macintosh) for which such compilers are available.
\end{abstract}




\section{TABLE OF CONTENTS}

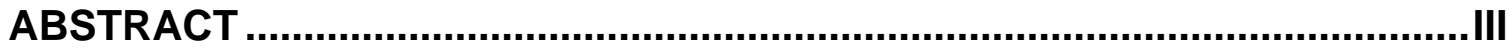

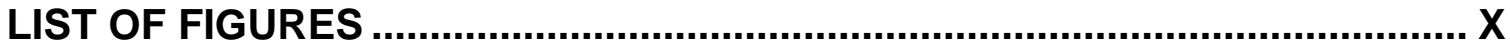

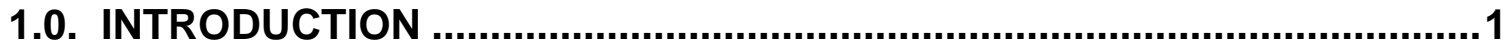

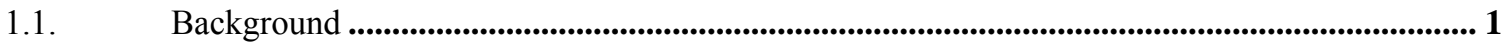

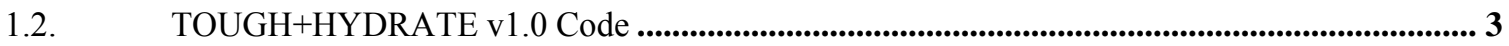

\subsection{CONCEPTS, UNDERLYING PHYSICS, AND GOVERNING EQUATIONS..9}

2.1. Modeled Processes and Underlying Assumptions....................................................................... 9

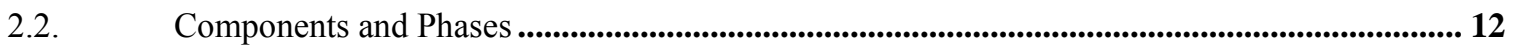

2.3. The Mass and Energy Balance Equation......................................................................................... 14

2.4. Mass Accumulation Terms ............................................................................................................ 14

2.5. Heat Accumulation Terms............................................................................................................. 17

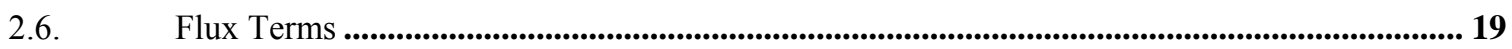

2.7. Source and Sink Terms............................................................................................................. 22

2.8. Thermophysical Properties.................................................................................................... 24

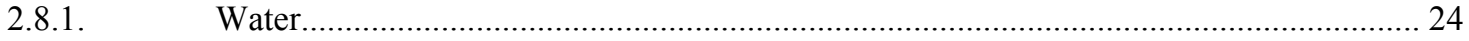

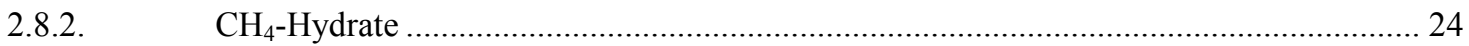

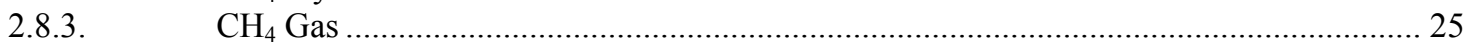

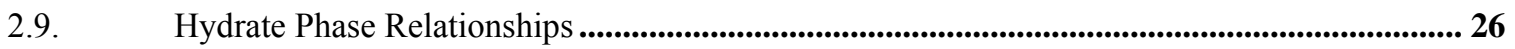

2.10. Inhibitor Effects on Hydrate Equilibrium.......................................................................................... 28

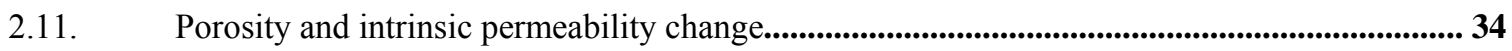

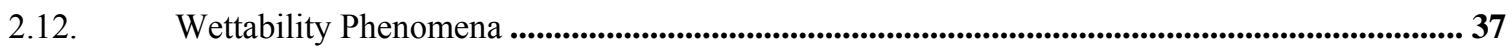

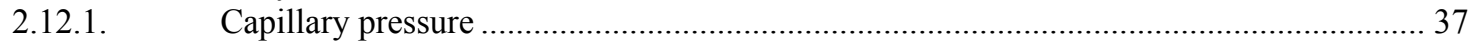

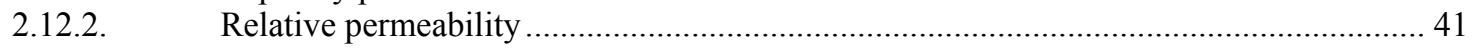

2.12.3. Pore compressibility of unconsolidated media in the presence of

$2.13 \quad$ Multiphase Diffusion................................................................................................................... 52

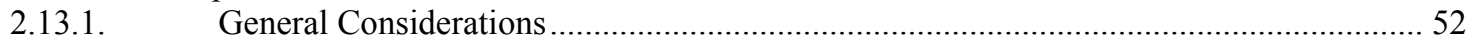

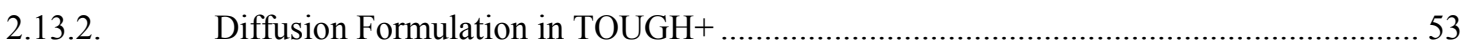

2.14. Description of Flow in Fractured Media .................................................................................. 54 


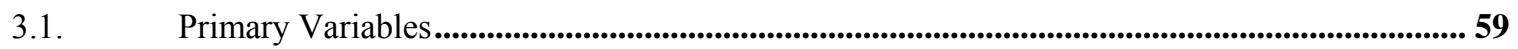

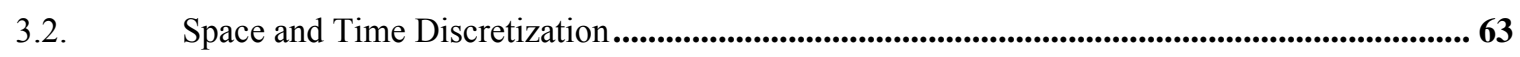

3.3. The Newton-Raphson Iteration ......................................................................................65

3.4. Implications of the Space Discretization Approach ...................................................................66

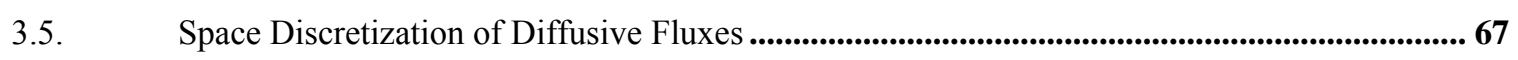

3.6. Compiling the TOUGH+HYDRATE Code ................................................................................. 69

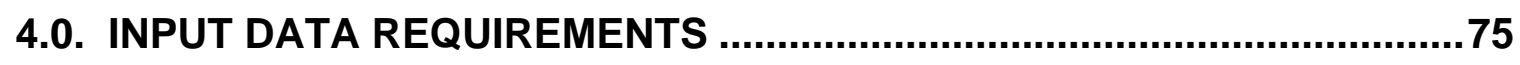

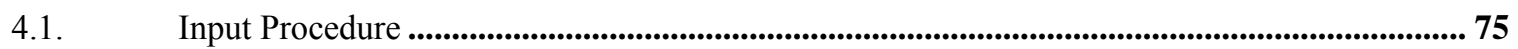

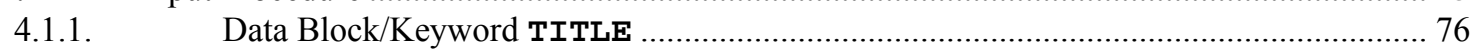

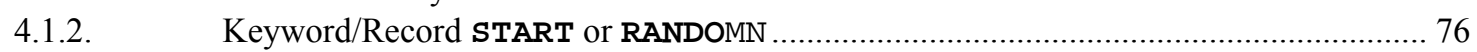

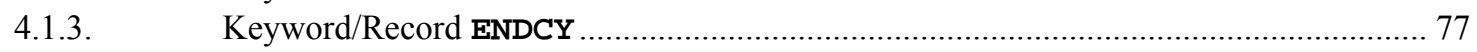

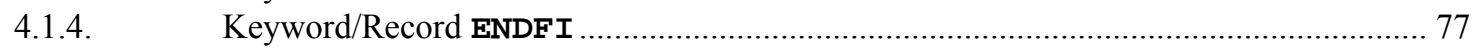

4.1.5. General Comments on the Structure of TOUGH+ Standard Input Files........................... 77

5.0. MEMORY SPECIFICATION AND ALLOCATION ...................................81

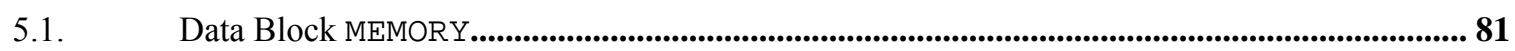

6.0. PHYSICAL PROPERTIES OF SYSTEM .................................................

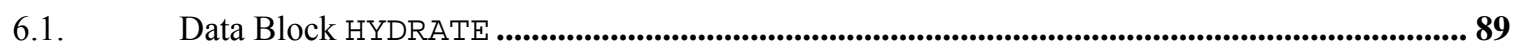

6.2. Data Block ROCKS or MEDIA............................................................................................ 92

6.3. Data Block RPCAP ....................................................................................................................... 98

6.3.1. Relative Permeability Functions ............................................................................. 98

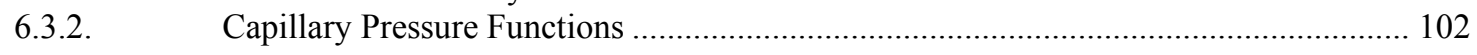

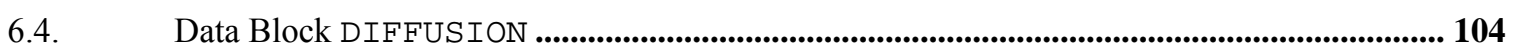

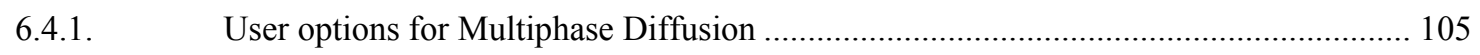

6.5. Block-by-Block Permeability Modification .......................................................................... 107

\subsection{GEOMETRICAL REPRESENTATION, DOMAIN DISCRETIZATION, AND GRID GENERATION ..........................................................................111}

7.1. TOUGH+ Convention for Geometrical Data ..................................................................... 111

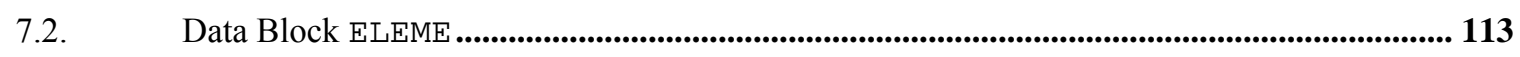

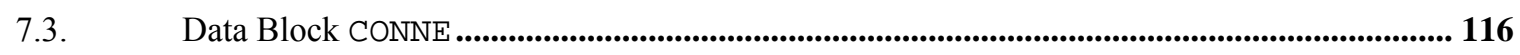


7.4. Data Block MESHM (Meshmaker) …....................................................................................... 118

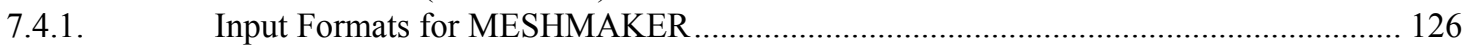

8.0. INITIAL CONDITIONS AND BOUNDARY CONDITIONS .......................139

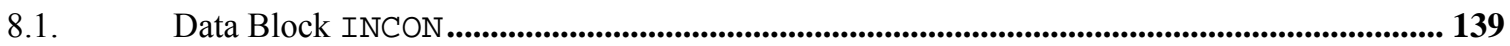

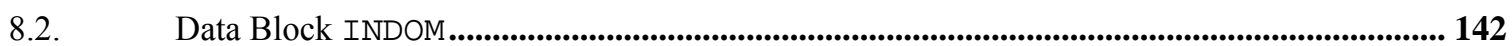

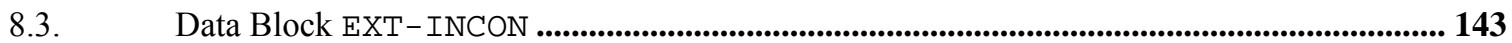

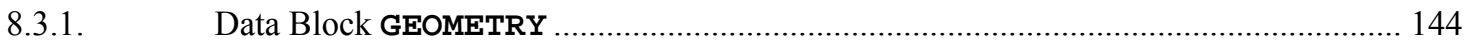

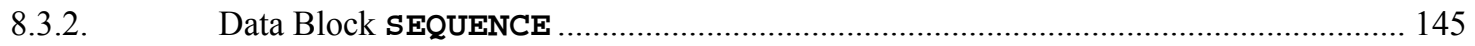

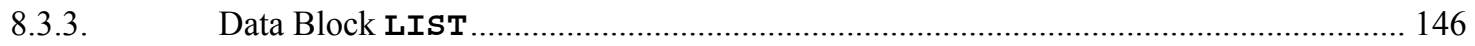

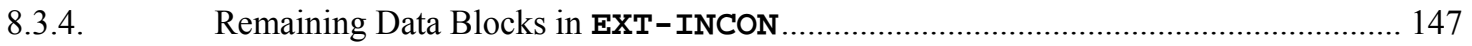

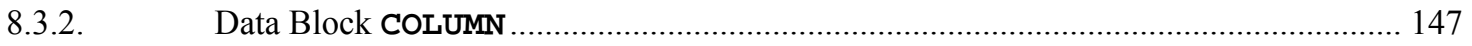

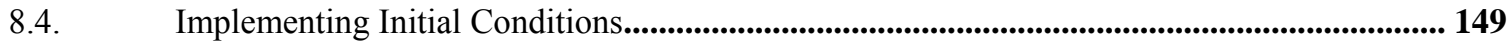

8.5. Implementing Boundary Conditions ......................................................................................... 151

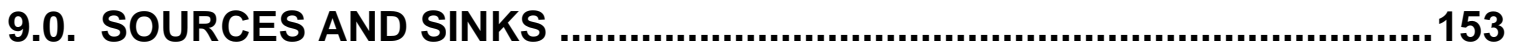

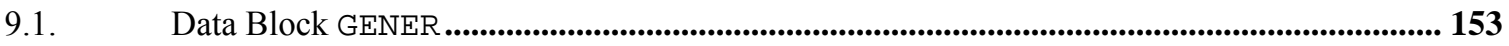

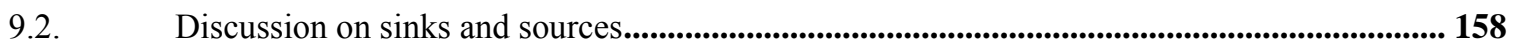

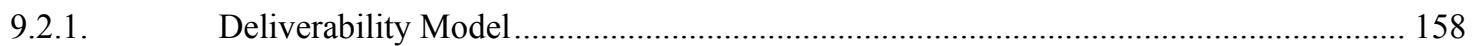

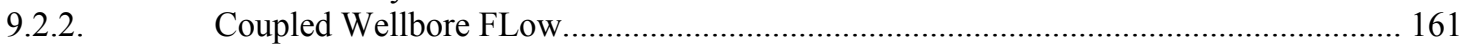

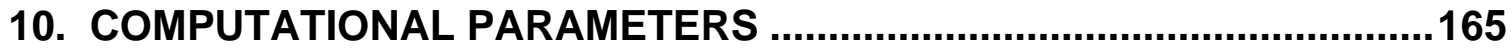

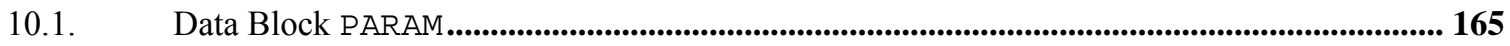

10.2. Data Block SOLVR ................................................................................................................ 179

10.3. Discussion on Linear Equation Solvers........................................................................................... 185

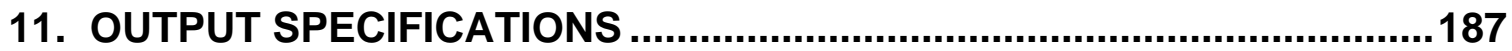

$11.1 \quad$ Output of Primary and Secondary Variables ................................................................................ 188

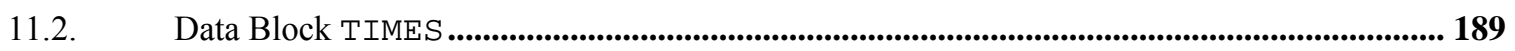

11.3. Data Block Elem_Time_Series or FOFT ...................................................................................... 190

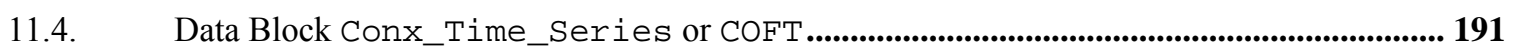

11.5. Data Block SS_Time_Series or GOFT......................................................................... 193

11.6. Data Block NOVER ....................................................................................................................... 194

11.7. Warning Output and Error Messages ................................................................................................ 194 
12.1. Example Files and Naming Conventions............................................................................. 199

12.2. Problem Test_1T: Thermal Stimulation, Equilibrium Dissociation, No Inhibitor...................... 200

12.3. Problem Test_1Tk: Thermal Stimulation, Kinetic Dissociation, No Inhibitor ........................... 206

12.4. Problems Test_1P: Depressurization, Equilibrium Dissociation, No Inhibitor .......................... 215

12.5. Problem Test_1Pk: Depressurization, Kinetic Dissociation, No Inhibitor ................................ 217

12.6. Problem Test_1P_Ice: Thermal Stimulation, Kinetic Dissociation, No Inhibitor, Ice Evolution

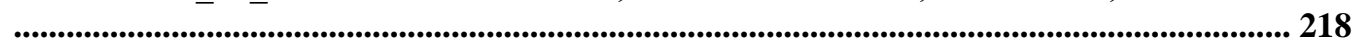

12.7. Problem Test_1TbS and Test_1TbSk: Hydrate Formation, Equilibrium and Kinetic Hydration reaction, Inhibitor .................................................................................................................................. 226

12.8. Problem Test_2Qp: Equilibrium Hydrate Dissociation, Depressurization, Radial Grid - Single Well ......................................................................................................................................... 230

12.9. Problem Test_3Qpk: Kinetic Hydrate Dissociation, Depressurization, Radial Grid - Single Well with Wellbore Heating................................................................................................................... 234

12.10. Problem Test_2D: Equilibrium Hydrate Dissociation, 2-D Areal System .................................. 238

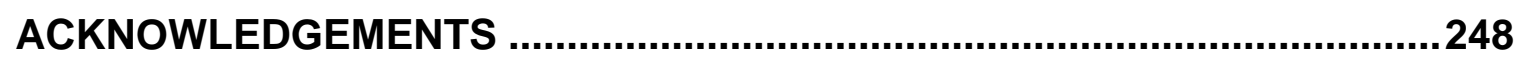

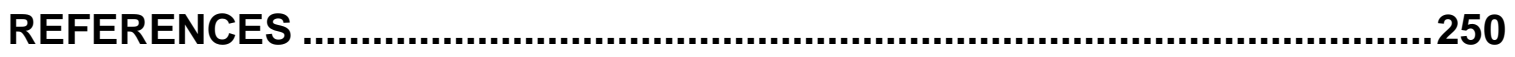

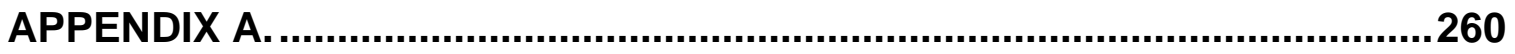

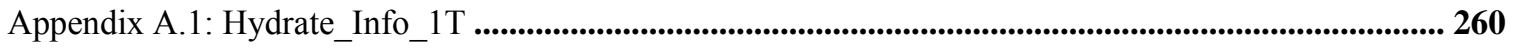

Appendix A.2: Conx_Time_Series_1T ...................................................................................................................... 262

Appendix A.3: Hydrate_Info_1P_Ice................................................................................................................ 262 


\section{List of Figures}

Figure 2.1. Relationship of the equilibrium hydration pressure $P e$ and temperature $T e$ of the $\mathrm{CH}_{4}$-hydrate [Moridis, 2003]

Figure 2.2. Modified $P e-T e$ relationship in the vicinity of the quadruple point

Figure 2.3. Temperature dependence of the $\mathrm{CH}_{4}$-hydrate heat of dissociation $\Delta H^{0}$ along the

(a) I-H-V and (b) $\mathrm{L}_{w}-\mathrm{H}-\mathrm{V}$ phase lines [Moridis, 2003].

Figure 2.4. Modified $\Delta H^{0}$ vs. Te relationship used in TOUGH+HYDRATE.

Figure 2.5. Pressure-temperature equilibrium relationship in the phase diagram of the water-- $\mathrm{CH} 4--$ hydrate system in $\mathrm{T}+\mathrm{H}$......

Figure 2.6. Schematic of pore channels, showing convergent-divergent geometry with a succession of pore throats and pore bodies.

Figure 2.7. Tubes-in-series model of pore channels

Figure 2.8. Schematic of probability density function $p(r)$ for pore size distribution.

Figure 2.9. Liquid and gas relative permeabilities based on the van Genuchten model [Finsterle, 1999].

Figure 2.10. Compressibility of an unconsolidated porous medium impregnated with cementing solid phases (ice and/or hydrates). In this example, $S_{S \min }=0.15$, $S_{S \max }=0.4, \alpha_{P U}=10^{-8} \mathrm{~Pa}^{-1}, \alpha_{P L}=10^{-9} \mathrm{~Pa}^{-1}$ and $\delta=0.015$.

Figure 2.11. Effect of the varying compressibility described in Figure 2.10 on the porosity of an unconsolidated porous medium undergoing depressurization for various levels of saturation $S_{S}$ of cementing solid phases.

Figure 2.12. Idealized double porosity model of a fractured porous medium.

Figure 2.13. Subgridding in the method of "multiple interacting continua" (MINC). 56

Figure 2.14. Flow connections in the "dual permeability" model. Global flow occurs between both fracture $(F)$ and matrix $(M)$ grid blocks. In addition there is F-M interporosity flow.

Figure 3.1. Space discretization and geometry data in the integral finite difference method

Figure 7.1. An example of a MeshMaker. $f 95$ input file for the creation of a Cartesian 3D grid. Note that no heterogeneous regions or boundaries are defined in this grid.

Figure 7.2. An example of a MeshMaker. $\mathrm{f} 95$ input file for the creation of a single-layer (1D) cylindrical grid. Note that no heterogeneous regions or boundaries are defined in this grid

Figure 7.3. An example of a MeshMaker. 995 input file for the creation of a large Cartesian 3D grid with heterogeneous regions and defined boundaries. 136 
Figure 7.4. An example of a MeshMaker. 995 input file for the creation of a large cylindrical 2D grid with multiple layers, heterogeneous regions and defined boundaries.

Figure 8.1. An example of the NAMELIST-described termination data printed at the end of the SAVE file from a TOUGH+ simulation. These data can be read as part of the INCON data block, or of the INCON external file. The names of the variables defined in the NAMELIST are self-explanatory. For reference, this figure lists the conditions in the last two elements (FJ000 and FJ106) of the grid in the TOUGH+ simulation.

Figure 10.1. An example of a Parameter_Update_File for parameter updating in the course of a TOUGH+ simulation. Within the namelists

(Real_Parameters_To_Update and

Integer_Parameters_To_Update), parameters can be entered in any order, data are read using any kind of appropriate format, only the needed parameters are included, and comments can be added.

Figure 10.2. An example of a Parameter_Update_File indicating three completed parameter updates, in addition to another one (at the top of the file) that has not yet been executed.

Figure 11.1. An example of the SUBDOMAINS data block for tracking the evolution of volume-averaged properties and conditions in specified subdomains. This data block uses namelist-based formats for data inputs.

Figure 12.1. Input file for example problem Test_1T (in Section 12.2) involving thermal stimulation, equilibrium dissociation, and no inhibitor.

Figure 12.2. Input file for example problem Test_1Tk (in Section 12.3) involving thermal stimulation, kinetic dissociation, and no inhibitor.

Figure 12.3. Comparison of the volumetric rates of $\mathrm{CH}_{4}$ release from hydrate dissociation in problems Test_1T and Test_1Tk.

Figure 12.4. Comparison of the cumulative volumes of $\mathrm{CH}_{4}$ released from hydrate dissociation in problems Test_1T and Test_1Tk

Figure 12.5. Comparison of the volumetric production rates of $\mathrm{CH}_{4}$ (measured as the flux crossing the $x=0$ boundary) in problems Test_1T and Test_1Tk.

Figure 12.6. Comparison of the total production volumes of $\mathrm{CH}_{4}$ (measured as the total volumes crossing the $x=0$ boundary) in problems Test_1T and Test_1Tk. ..... 214

Figure 12.7. Input file for example problem Test_1Pk (in Section 12.4) involving depressurization, equilibrium dissociation, and no inhibitor.

Figure 12.8. Comparison of the volumetric rates of $\mathrm{CH}_{4}$ release from depressurizationinduced hydrate dissociation in problems Test_1P, Test_1Pk and Test_1P_ice.

Figure 12.9. Comparison of the cumulative volumes of $\mathrm{CH}_{4}$ released from depressurization-induced hydrate dissociation in problems Test_1P, Test_1Pk and Test_1P_ice. 
Figure 12.10. Comparison of the volumetric production rates of $\mathrm{CH}_{4}$ (measured as the flux crossing the $x=0$ boundary) in problems Test_1P, Test_1Pk and Test_1P_ice.

Figure 12.11. Comparison of the total production volumes of $\mathrm{CH}_{4}$ (measured as the total volumes crossing the $x=0$ boundary) in problems Test_1P, Test_1Pk and Test_1P_ice.

Figure 12.12. Comparison of the volumetric rates of $\mathrm{CH}_{4}$ consumption during hydrate formation in problems Test_1TbS, Test_1TbSk and Test_1TbS2.

Figure 12.13. Comparison of the cumulative volumes of $\mathrm{CH}_{4}$ consumed during hydrate formation in problems Test_1TbS, Test_1TbSk and Test_1TbS2.

Figure 12.14. Volumetric rate of $\mathrm{CH}_{4}$ release during hydrate dissociation in problem Test_2Qp.

Figure 12.15. Cumulative volume of $\mathrm{CH}_{4}$ released during hydrate dissociation in problem Test_2Qp.

Figure 12.16. Volumetric rates of $\mathrm{CH}_{4}(\mathrm{a})$ release in the reservoir during hydrate dissociation and (b) production from the well in problem Test_30pk

Figure 12.17. Cumulative volumes of $\mathrm{CH}_{4}$ (a) released in the reservoir during hydrate dissociation and (b) produced from the well in problem Test_3Qpk.

Figure 12.18. Volumetric rates of $\mathrm{CH}_{4}$ release from the hydrate in the reservoir during gas production in problem Test_2D.

Figure 12.19. Pressure distribution in the reservoir at $t=2$ days in problem Test_2D.......

Figure 12.20. Temperature distribution in the reservoir at $t=2$ days in problem Test_2D.

Figure 12.21. Hydrate saturation distribution in the reservoir at $t=2$ days in problem Test_2D.

Figure 12.22. Aqueous phase saturation distribution in the reservoir at $t=2$ days in problem Test_2D.

Figure 12.23. Gas saturation distribution in the reservoir at $t=2$ days in problem Test_2D. 


\section{List of Tables}

Table 3.1. Primary Variables in Equilibrium Hydrate Simulations without Inhibitor ${ }^{*} \ldots \ldots \ldots \ldots \ldots \ldots . . . . . . .61$

Table 3.2. Primary Variables in Kinetic Hydrate Simulations without Inhibitor* ........................ 62

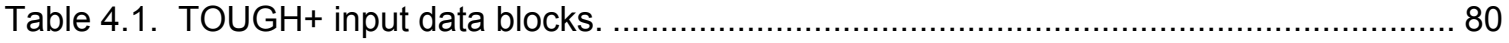




\subsection{Introduction}

\subsection{Background}

Gas hydrates are solid crystalline compounds in which gas molecules are encaged inside the lattices of ice crystals. These gases are referred to as guests, whereas the ice crystals are called hosts. Of particular interest are hydrates in which the gas is a hydrocarbon. Under suitable conditions of low temperature and high pressure, a hydrocarbon gas $M$ will react with water to form hydrates according to

$$
\mathrm{M}+\mathrm{N}_{\mathrm{H}} \mathrm{H}_{2} \mathrm{O}=\mathrm{M} \cdot \mathrm{N}_{\mathrm{H}} \mathrm{H}_{2} \mathrm{O}
$$

where $N_{H}$ is the hydration number.

Vast amounts of hydrocarbons are trapped in hydrate deposits [Sloan, 1998]. Such deposits exist where the thermodynamic conditions allow hydrate formation, and are concentrated in two distinctly different types of geologic formations where the necessary low temperatures and high pressures exist: in the permafrost and in deep ocean 
sediments. The lower depth limit of hydrate deposits is controlled by the geothermal gradient.

Current estimates of the worldwide quantity of hydrocarbon gas hydrates range between $10^{15}$ to $10^{18} \mathrm{~m}^{3}$. Even the most conservative estimates of the total quantity of gas in hydrates may surpass by a factor of two the energy content of the total fuel fossil reserves recoverable by conventional methods. The magnitude of this resource could make hydrate reservoirs a substantial future energy resource. While current economic realities do not favor gas production from the hydrate accumulations, their potential clearly demands evaluation.

The majority of naturally occurring hydrocarbon gas hydrates contain $\mathrm{CH}_{4}$ in overwhelming abundance. Simple $\mathrm{CH}_{4}$-hydrates concentrate methane volumetrically by a factor of 164 , and require less than $15 \%$ of the recovered energy for dissociation. Natural $\mathrm{CH}_{4}$-hydrates crystallize mostly in the I structure, which contains $46 \mathrm{H}_{2} \mathrm{O}$ molecules per unit cell. They have a $N_{H}$ ranging from 5.77 to 7.41 , with $N_{H}=6$ being the average hydration number and $N_{H}=5.75$ corresponding to complete hydration [Sloan, 1998].

There are three main methods of hydrocarbon recovery from gas hydrates: (a) thermal stimulation [McGuire, 1981], in which gas release is effected by heating the hydrate above the dissociation temperature at a given pressure, (b) depressurization [Holder et al., 1982], in which the gas release is achieved by lowering the pressure below that of the hydrate stability, and (c) 'inhibitor' injection [Kamath and Godbole, 1987] in which the hydrocarbon is produced after the injection of substances (e.g., brines, alcohols) that destabilize the hydrate. Combinations of these methods can also be used. 
Numerical codes and studies on the simulation of gas production from dissociating hydrates are limited. Drenth and Swinkels [2000] developed a four-component, threephase numerical model for the equilibrium dissociation of binary hydrates in marine environments. They provided an in-depth discussion of the challenges facing production from gas hydrates and identified knowledge gaps in numerical simulation of gas production from hydrate dissociation. Kurihara et al. [2003] developed a numerical simulator, and used it for the prediction of gas production from gas hydrates from both marine and permafrost hydrate deposits.

Moridis et al. [1998] developed EOSHYDR, a TOUGH2 [Pruess et al., 1999] module for the simulation of dissociating simple methane hydrates under equilibrium conditions in both permafrost and marine accumulations. Moridis et al. [2003] enhanced EOSHYDR and developed EOSHYDR2 - also a TOUGH2 module - for the simulation of binary hydrates reacting under both equilibrium and kinetic conditions. TOUGH2/EOSHYDR2 was used for the simulation of gas production from hydrates under a variety of geologic and thermodynamic conditions, and involving various production strategies [Moridis, 2003; Moridis et al., 2004; Moridis and Collett, 2004].

\subsection{TOUGH+HYDRATE v1.0 Code}

TOUGH+HYDRATE v1.0 is a new code for the simulation of the behavior of hydrate-bearing geologic systems. TOUGH+HYDRATE (hereafter often referred to as $\mathbf{T}+\mathbf{H}$ for brevity) is the first member of TOUGH+, the successor to the TOUGH2 [Pruess et al., 1991] family of codes for multi-component, multiphase fluid and heat flow

developed at the Lawrence Berkeley National Laboratory. It is written in standard 
FORTRAN 95/2003 to take advantage of all the object-oriented capabilities and the enhanced computational features of that language.

While the underlying principles, physics and thermodynamics are similar to those used in TOUGH2/EOSHYDR2 [Moridis, 2003], the code in $\mathbf{T}+\mathbf{H}-$ and in the earlier, transitional version of the code named TOUGH-Fx/HYDRATE [Moridis et al., 2005], of which the $\mathbf{T}+\mathbf{H}$ code is a natural extension - was completely rewritten and its architecture is drastically different. The new code employs dynamic memory allocation, thus minimizing storage requirements. It follows the tenets of Object-Oriented Programming (OOP), and involves entirely new data structures that describe the objects upon which the code is based. The basic objects are defined through derived data types, and their properties and processes are described in modules and sub-modules, i.e., entities that incorporate the object attributes and parameters in addition to the procedures (corresponding to the older concepts of "functions" and "subroutines" in FORTRAN 77) that describe its behavior and processes. The $\mathrm{TOUGH}+$ code is based on a completely modular structure that is designed for maximum traceability and ease of expansion.

By using the capabilities of the FORTRAN95/2003 language, the new OOP architecture involves the use of pointers, lists and trees, data encapsulation, defined operators and assignments, operator extension and overloading, use of generic procedures, and maximum use of the powerful intrinsic vector and matrix processing operations (available in the extended mathematical library of FORTRAN 95/2003). This leads to increased computational efficiency, while allowing seamless applicability of the code to multi-processor parallel computing platforms. The result is a code that is transparent and compact, and frees the developer from the tedium of tracking the 
disparate attributes that define the objects, thus enabling a quantum jump in the complexity of problem that can be tackled. This is demonstrated in $\mathbf{T}+\mathbf{H}$, in which 26 different phase combinations can be easily described. An additional feature of the FORTRAN 95/2003 language of TOUGH+ is the near complete interoperability with $\mathrm{C} / \mathrm{C}++$, which allows the interchangeable use of procedures written in either FORTRAN 95/2003 or $\mathrm{C} / \mathrm{C}++$, and makes possible the seamless interaction with pre- and postprocessing graphical environments.

Based on insights provided by TOUGH2/EOSHYDR2 [Moridis, 2003], numerical 'bottlenecks' were removed, different (and fewer) primary variables were selected, and more powerful linearization techniques were employed in $\mathbf{T}+\mathbf{H}$, resulting in significant improvements in execution speed and numerical performance. Note that TOUGH+HYDRATE v1.0 still uses most of the inputs (and the input formats) used by the conventional TOUGH2 code to fulfill the functional requirement (part of the code design) of backward compatibility of the $\mathrm{TOUGH}+$ family codes with older input data files used in TOUGH2 [Pruess et al., 1999] simulations. More powerful input data file structures will be available in future releases of $\mathrm{TOUGH}+$.

By solving the coupled equations of mass and heat balance, $\mathbf{T}+\mathbf{H}$ can model the nonisothermal gas release, phase behavior and flow of fluids and heat in complex geologic media. The code can simulate production from natural $\mathrm{CH}_{4}$-hydrate deposits in the subsurface (i.e., in the permafrost and in deep ocean sediments), as well as laboratory experiments of hydrate dissociation/formation in porous/fractured media. The only limitations on the size of the domain to be simulated are imposed by the underlying physics. Thus, if the volume of the domain and its subdivision are such that (a) a 
representative volume can be defined and (b) Darcy's law applies, then $\mathbf{T}+\mathbf{H}$ can be used for the prediction of the behavior of a hydrate-bearing geological system.

TOUGH+HYDRATE v1.0 includes both an equilibrium and a kinetic model of hydrate formation and dissociation. The model accounts for heat and up to four mass components, i.e., water, $\mathrm{CH}_{4}$, hydrate, and water-soluble inhibitors such as salts or alcohols. These are partitioned among four possible phases (gas phase, liquid phase, ice phase and hydrate phase). Hydrate dissociation or formation, phase changes and the corresponding thermal effects are fully described, as are the effects of inhibitors. The model can describe all possible hydrate dissociation mechanisms, i.e., depressurization, thermal stimulation, salting-out effects and inhibitor-induced effects, both singly and in any combination.

Compared to TOUGH2/EOSHYDR2, $\mathbf{T}+\mathbf{H}$ no longer tracks individually pre-existing free gas and gas from dissociation. While this was a convenient feature in TOUGH2/EOSHYDR2, it was not scientifically robust (because of the empirical and arbitrary differentiation between "native" and "hydrate-originating" gas), caused significant problems in complex simulations involving successive hydrate formation and dissociation (because of the difficulty of assigning gas provenance under these conditions), introduced an unacceptable convergence behavior (brought about by the empirical gas differentiation), increased the number of equations and introduced disproportionately large additional computational work (caused by the increased size of the problem and the demanding computational process for the estimation of binary gas diffusion). Elimination of the gas tracking option in $\mathbf{T}+\mathbf{H}$ greatly enhanced the convergence behavior, while reducing execution times by up to $50 \%$. 
While the TOUGH2/EOSHYDR2 capabilities to describe binary hydrates, and the properties and behavior of all hydrate forming gases are fully implemented in $\mathbf{T}+\mathbf{H}$, only methane hydrates can be simulated by this version of the code. The reason for this stems from the fact that our ability to mathematically describe the problem far exceeds the supporting fundamental knowledge on the subject. Treating the component hydrates as individual entities unaffected by each other or as segregated macroscopic quantities of hydrocarbon within the hydrate is incorrect because binary hydrates behave in a manner akin to solid solutions [Sloan, 1998]. Thus, changes in the composition of the hydrate and in the gas phase are functions not only of pressure and temperature, but also of concentration. Currently, there are no readily available P-T-X diagrams for such hydrates. The empirical distribution coefficient $K_{v s i}$ method of Carson and Katz [1942] employed in TOUGH2/EOSHYDR2 was difficult, and often impossible, to converge (the highest convergence rate was $65 \%$, and was observed in very simple problems), and lead to long execution times. The most physically and mathematically robust approach is to incorporate fast regression relationships based on the computationally intensive but conceptually sound statistical thermodynamics approach of Sloan [1998]. This option is currently being explored for incorporation into later version of the code if such a need arises.

Scoping simulations had indicated that sometimes fully $80 \%$ of the computational load in TOUGH2/EOSHYDR2 was due to the challenges of the distribution coefficient method. Given the fact that most of the most obvious hydrate deposit targets for gas production involve almost exclusively pure $\mathrm{CH}$-hydrates led to the decision to inactivate the binary hydrate capabilities in $\mathbf{T}+\mathbf{H}$. 
This report provides a detailed presentation of the features and capabilities of $\mathbf{T}+\mathbf{H}$, and includes a thorough discussion of the underlying physical, thermodynamic and mathematical principles of the model in addition to the main governing equations. The various phase regimes and the corresponding primary variables are discussed in detail, as well as the reasons for their selection. Examples of input data files (and of sections of the corresponding output files) are included as an aide to the $\mathbf{T}+\mathbf{H}$ user. Results from simulations of gas production from realistic hydrate-bearing geologic systems are also included. 


\subsection{Concepts, Underlying Physics, and Governing Equations}

\subsection{Modeled Processes and Underlying Assumptions}

$\mathbf{T}+\mathbf{H}$ can model the following processes and phenomena in hydrate-bearing geologic systems:

(1) The partitioning of the mass components among the possible phases

(2) The flow of gases and liquids in the geologic system

(3) The corresponding heat flow and transport

(4) Heat exchanges due to
a. Conduction
b. Advection/convection
c. Radiation
d. Hydrate reaction (dissociation or formation) 
e. Latent heat related to phase changes (ice melting or water fusion, water evaporation or vapor condensation)

f. Gas dissolution

g. Inhibitor dissolution

(5) Equilibrium or kinetic hydration reaction (dissociation or formation),

(6) The transport of water-soluble gases and inhibitors (such as salts and alcohols), accounting for advection and molecular diffusion

(7) The effects of water-soluble inhibitors on the hydrate behavior

(8) Any method of hydrate dissociation (i.e., depressurization, thermal stimulation and inhibitor effects), and combinations thereof

A deliberate effort was made to keep the simplifying assumptions involved in the development of the underlying physical, thermodynamic and mathematical model to a minimum. These include:

(1) Darcy's law is valid in the simulated domain under the conditions of the study.

(2) In the transport of dissolved gases and inhibitors, mechanical dispersion is small compared to advection (by neglecting mechanical dispersion, memory requirements and execution times are substantially reduced).

(3) The compressibility and thermal expansivity of hydrate are the same as those of ice (necessitated by dearth of data on the subject).

(4) The movement of the geologic medium (soil heaving) while freezing is not described, and the effects on pressure (caused by density differences between the liquid and ice phases) are accommodated through a relatively high pore compressibility of the geologic medium. 
(5) Dissolved salts do not precipitate as their concentration increases during water freezing. Consequently, the aqueous phase is not allowed to disappear when salts are present. This simplification was necessitated by (a) the marginal practical aspects of such a scenario, (b) the lack of fundamental knowledge and quantitative relationships describing the hydrate-salt interaction under these conditions, and (c) the computationally intensive requirements of describing the thermodynamics of dense brines and halite precipitation.

(6) The concentration of the dissolved inhibitors is such that it does not affect the thermophysical properties of the aqueous phase. Although the thermodynamics of inhibitor-water systems are well known and are already available within $\mathbf{T}+\mathbf{H}$, they are not invoked in this version (but will be considered as an option in future code releases) because their effect may not very important, they are computationally intensive, and inhibitor-induced dissociation is not considered an attractive first option for gas production from hydrate deposits.

(7) The inhibitor is non-volatile in the temperature-pressure range of the study. This simplification was introduced because of the limited practical possibility for such a scenario, and of the significant computational requirements to account for the inhibitor vapor pressure and diffusion in the gas phase.

(8) The pressure $P<100 \mathrm{MPa}(6800 \mathrm{psi})$. The pressure-dependent equations describing the hydrate properties and behavior in $\mathbf{T}+\mathbf{H}$ provide accurate solutions for a $P$ over nine times larger than the largest pressure at which natural gas hydrates are known to exist (i.e., about $11 \mathrm{MPa}$ ). Thus, the existing capabilities can easily accommodate any natural or laboratory hydrate system. 
Although equations for an accurate description of the thermophysical properties of hydrate systems for $P$ as high as $1000 \mathrm{MPa}$ are available in the code, this option is disabled because it involves an iterative process that increases the execution time by a factor of 3 or 4 even for $P<100 \mathrm{MPa}$.

\subsection{Components and Phases}

Depending on the thermodynamic state of the system, the amount of $\mathrm{CH}_{4}$-hydrate created or $\mathrm{CH}_{4}$ gas released is determined from the reaction

$$
\mathrm{CH}_{4}+\mathrm{N}_{m} \mathrm{H}_{2} \mathrm{O}=\mathrm{CH}_{4} \cdot \mathrm{N}_{m} \mathrm{H}_{2} \mathrm{O},
$$

where $N_{H}$ is the hydration number, and the subscript $m$ denotes methane. In addition to simple $\mathrm{CH}_{4}$-hydrates, natural hydrates may include one or more additional gasses. Such hydrate-forming gases exist practically in all natural hydrates and can play a significant role in their nucleation and behavior. The reaction describing the formation/hydration of a composite (binary) hydrate is

$\chi_{m}\left[\mathrm{CH}_{4} \cdot \mathrm{N}_{m} \mathrm{H}_{2} \mathrm{O}\right]+\chi_{G}\left[G \cdot \mathrm{N}_{G} \mathrm{H}_{2} \mathrm{O}\right]=\chi_{m} \mathrm{CH}_{4}+\chi_{G} G+\left(\chi_{m} N_{m}+\chi_{G} N_{G}\right) H_{2} \mathrm{O}$,

where $G$ is the second hydrate-forming gas, $N$ is the hydration number, $\chi$ is the mole fraction in the binary hydrate, and the subscripts $m$ and $G$ denote the methane and the second gas, respectively. Obviously, $\chi_{m}+\chi_{G}=1$. The gas $G$ can be one of $\mathrm{CO}_{2}, \mathrm{H}_{2} \mathrm{~S}, N_{2}$, or another gaseous alkane $C_{n} H_{2 n+2}(m=2,3,4)$. In permafrost hydrate accumulations, $\chi_{m}$ routinely exceeds 95\% [Collett et al., 1999].

A non-isothermal hydrate system can be fully described by the appropriate mass balance equations and an energy balance equation. The following components $\kappa$ (and the 
corresponding indicators used in the subsequent equations), corresponding to the number of equations, are considered in $\mathbf{T}+\mathbf{H}$ :

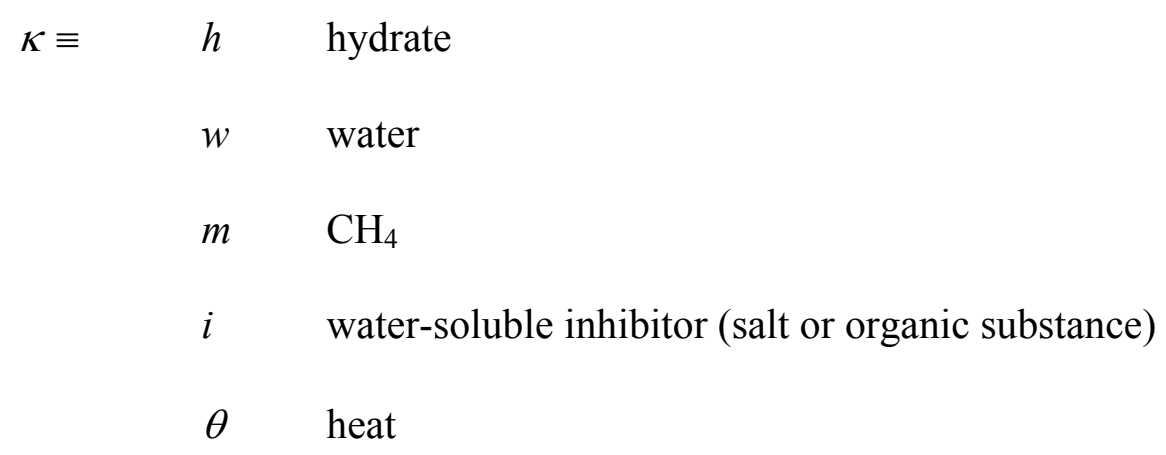

These mass and energy components are partitioned among four possible phases $\beta$, which are listed below along with the corresponding indicators (used in the subsequent equations):

$$
\begin{array}{cl}
\beta \equiv \quad & \text { solid-hydrate (components: } m, w \text { - equilibrium, } h \text { - kinetic) } \\
A & \text { aqueous (components: dissolved } m \text {, dissolved } i \text { ) } \\
G & \text { gaseous (components: } m \text {, vapor } w \text { ) } \\
I & \text { solid-ice (component: } w \text { ) }
\end{array}
$$

Note that hydrate is both a component and a phase under kinetic hydration reaction conditions. Under equilibrium conditions, hydrate is treated only as a phase. 


\subsection{The Mass and Energy Balance Equation}

Following Pruess et al. [1999], mass and heat balance considerations in every subdomain (gridblock) into which the simulation domain is been subdivided by the integral finite difference method dictates that

$$
\frac{d}{d t} \int_{V_{n}} M^{\kappa} d V=\int_{\Gamma_{n}} \mathbf{F}^{\kappa} \cdot \mathbf{n} d \Gamma+\int_{V_{n}} q^{\kappa} d V,
$$

where:

$V, V_{n} \quad$ volume, volume of subdomain $n\left[L^{3}\right]$;

$M^{\kappa} \quad$ mass accumulation term of component $\kappa\left[\mathrm{kg} \mathrm{m}^{-3}\right]$;

$A, \Gamma_{n}$ surface area, surface area of subdomain $n\left[L^{2}\right]$;

$\mathbf{F}^{\kappa} \quad$ Darcy flux vector of component $\kappa\left[\mathrm{kg} \mathrm{m}^{-2} \mathrm{~s}^{-1}\right]$;

n inward unit normal vector;

$q^{\kappa} \quad$ source/sink term of component $\kappa\left[\mathrm{kg} \mathrm{m}^{-3} \mathrm{~s}^{-1}\right]$;

$t \quad$ time $[T]$.

\subsection{Mass Accumulation Terms}

Under equilibrium conditions, the mass accumulation terms $M^{\kappa}$ for the mass components in equation (2.3) are given by

$$
M^{\kappa}=\sum_{\beta \equiv A, G, I} \phi S_{\beta} \rho_{\beta} X_{\beta}^{\kappa}, \quad \kappa \equiv w, m, i
$$

where

$\phi \quad$ porosity [dimensionless];

$\rho_{\beta} \quad$ density of phase $\beta\left[\mathrm{kg} \mathrm{m}^{-}\right]$; 
$S_{\beta} \quad$ saturation of phase $\beta$ [dimensionless $]$

$X_{\beta}^{\kappa} \quad$ mass fraction of component $\kappa \equiv w, m, h$ in phase $\beta[\mathrm{kg} / \mathrm{kg}]$

In the equilibrium model, different cases of $\beta$ give the following relations:

$$
\begin{aligned}
& \beta \equiv A: X_{A}^{h}=0 \\
& \beta \equiv G: X_{G}^{h}=X_{G}^{i}=0 \\
& \beta \equiv H: X_{H}^{w}=\frac{W^{m}}{W^{h}}, X_{M}^{m}=1-X_{H}^{w}, X_{H}^{i}=0, X_{H}^{h}=0 \\
& \beta \equiv I: X_{I}^{m}=X_{I}^{h}=X_{I}^{i}=0, X_{I}^{w}=1
\end{aligned}
$$

The terms $W^{m}$ and $W^{h}$ denote the molecular weights of the $\mathrm{CH}_{4}$ and of the hydrate, respectively. Thus, the values of $X_{H}^{w}$ and $X_{M}^{m}$ reflect the stoichiometry in Equation (2.1).

Under kinetic conditions, the mass accumulation terms $M^{\kappa}$ in Equation (2.3) are given by

$$
M^{\kappa}=\sum_{\beta \equiv A, G, H, I} \phi S_{\beta} \rho_{\beta} X_{\beta}^{\kappa}, \kappa \equiv w, m, h, i
$$

In the kinetic model, different cases of $\beta$ give the following relations

$$
\begin{aligned}
& \beta \equiv A: X_{W}^{h}=0 \\
& \beta \equiv G: X_{G}^{h}=X_{G}^{i}=0 \\
& \beta \equiv H: X_{H}^{w}=X_{H}^{m}=X_{H}^{i}=0 \\
& \beta \equiv I: X_{I}^{m}=X_{I}^{h}=X_{I}^{i}=0, \quad X_{I}^{w}=1
\end{aligned}
$$

Under kinetic conditions, the equation describing the behavior of the hydrate mass component and phase is provided by the model of [Kim et al., 1987] as

$$
Q_{H}=\frac{\partial M}{\partial t}=-K_{0} \exp \left(\frac{\Delta E_{a}}{R T}\right) F_{A} A\left(f_{e q}-f_{v}\right),
$$

where

$K_{0} \quad$ intrinsic hydration reaction constant $\left[\mathrm{kg} \mathrm{m}^{-2} \mathrm{~Pa}^{-1} \mathrm{~s}^{-1}\right]$; 
$\Delta E a$ hydration activation energy $\left[\mathrm{J} \mathrm{mol}^{-1}\right]$;

$R \quad$ universal gas constant $\left[8.314 \mathrm{~J} \mathrm{~mol}^{-1} \mathrm{~K}^{-1}\right]$;

$T \quad$ temperature $\left[{ }^{\circ} \mathrm{K}\right]$;

$F_{A} \quad$ area adjustment factor [dimensionless];

A surface area participating in the reaction $\left[\mathrm{m}^{2}\right]$;

$f_{e q} \quad$ fugacity at equilibrium at temperature $T(\mathrm{~Pa})$

$f_{v} \quad$ fugacity in the gas phase at temperature $T(\mathrm{~Pa})$

The surface area is computed by assigning the hydrate saturation uniformly to the interstitial spaces of the porous medium. To accomplish this, the original solid grain volume (considered to be composed of spherical particles) is determined as $V_{p}=4 / 3 \pi r_{p}^{3}$, where $r_{p}$ is the solid grain radius [m]. Then, the number of voids $N_{V}$ (pore spaces) is assumed to be equal to the number of solid grains (a valid approach for spherical particles), and the corresponding void volume $V_{V}$ is computed from

$$
N_{V}=\frac{(1-\phi)}{V_{p}}, \quad V_{V}=\frac{\phi}{N_{V}},
$$

At the interface of pores and voids, the grain surface area is the same for both the grains and the voids, and is computed as $A_{p}=4 \pi r_{p}^{2}$, resulting in a total area (per unit volume) of $A_{T V}=N_{V} A_{p}$. Then the volume of the void is assumed to vary linearly with the $r_{V}^{3}$, where $r_{V}=0.1547 r_{p}$, is a representative radius describing the radius of the sphere fitting in the interstitial space between the spherical grains. Then, at any time $t$, a representative hydrate particle radius $r_{H}$ and volume $V_{H}$ are computed as

$$
V_{H}=\frac{\phi S_{H}}{N_{V}}, \quad r_{h}=r_{V}\left(\frac{V_{H}}{V_{V}}\right)^{1 / 3}=r_{V} S_{H}^{1 / 3}
$$


and the hydrate reactive area is computed as

$$
A=f_{A} A_{T V}\left(\frac{r_{H}}{r_{V}}\right)^{2}=f_{A} N_{V}\left(4 \pi r_{p}^{2}\right) S_{H}^{2 / 3} .
$$

The area adjustment factor $f_{A}$ accounts for the deviation of the interstitial volume from that based on the assumption of grain sphericity, and can incorporate heterogeneity effects related to the hydrate "particle" size and saturation distribution. Given the intrinsic permeability $k$ of a porous medium, the Kozeny-Carman equation can provide an estimate of the average (effective) radius $r_{p}$ of the porous medium grains as [Bear, 1972]

$$
r_{p}=\left[45 k \frac{(1-\phi)^{2}}{\phi^{3}}\right]^{1 / 2}
$$

Alternatively, an estimate of $r_{p}$ can be obtained from sieve analysis (if such data are available).

\subsection{Heat Accumulation Terms}

The heat accumulation term includes contributions from the rock matrix and all the phases, and, in the kinetic model, is given by the equation

$$
M^{\theta}=(1-\phi) \rho_{R} C_{R} T+\sum_{\beta=A, G, H, I} \phi S_{\beta} \rho_{\beta} U_{\beta}+Q_{\text {diss }},
$$

where

$$
Q_{\text {diss }}= \begin{cases}\Delta\left(\phi \rho_{H} S_{H} \Delta H^{0}\right) & \text { for equilibrium dissociation } \\ 0 & \text { for kinetic dissociation }\end{cases}
$$

$\rho_{R} \quad$ rock density $\left[\mathrm{kg} \mathrm{m}^{-3}\right]$;

$C_{R} \quad$ heat capacity of the dry rock $\left[\mathrm{J} \mathrm{kg}^{-1} \mathrm{~K}^{-1}\right]$;

$U_{\beta} \quad$ specific internal energy of phase $\beta\left[\mathrm{J} \mathrm{kg}^{-1}\right]$; 
$\Delta()$ change in the quantity in parentheses over the current time step;

$\Delta U_{H} \quad$ specific enthalpy of hydrate dissociation/formation $\left[\mathrm{J} \mathrm{kg}^{-1}\right]$

The specific internal energy of the gaseous phase is a very strong function of composition, is related to the specific enthalpy of the gas phase $H_{G}$, and is given by

$$
U_{G}=\sum_{\kappa=w, m} X_{G}^{\kappa} u_{G}^{\kappa}+U_{d e p}\left(=H_{G}-\frac{P}{\rho_{G}}\right),
$$

where $u_{G}^{\kappa}$ is the specific internal energy of component $\kappa$ in the gaseous phase, and $U_{d e p}$ is the specific internal energy departure of the gas mixture $\left[\mathrm{J} \mathrm{kg}^{-1}\right]$.

The internal energy of the aqueous phase accounts for the effects of gas and inhibitor solution, and is estimated from

$$
U_{A}=X_{A}^{w} u_{A}^{w}+X_{A}^{m}\left(u_{A}^{m}+U_{s o l}^{m}\right)+X_{A}^{i}\left(u_{A}^{i}+U_{s o l}^{i}\right),
$$

where $u_{A}^{w}, u_{A}^{m}$ and $u_{A}^{i}$ are the specific internal energies of $\mathrm{H}_{2} \mathrm{O}, \mathrm{CH}_{4}$ and the inhibitor at the conditions prevailing in the aqueous phase, respectively, and $U_{\text {sol }}^{m}$ and $U_{\text {sol }}^{i}$ are the specific internal energies corresponding to the dissolution of $\mathrm{CH}_{4}$ and of the inhibitor in water, respectively. The terms $u_{A}^{i}$ and $U_{H}$ are determined from

$$
u_{A}^{i}=h_{A}^{i}-\frac{P}{\rho_{i}}=\int_{T_{0}}^{T} C_{i} d T-\frac{P}{\rho_{i}} \text { and } U_{H}=H_{H}-\frac{P}{\rho_{H}}=\int_{T_{0}}^{T} C_{H} d T-\frac{P}{\rho_{H}}
$$

where $T_{0}$ is a reference temperature, $h_{A}^{i}$ and $H_{H}$ are the specific enthalpies of $\mathrm{H}_{2} \mathrm{O}$ and hydrate (phase or component), respectively, and $C_{i}$ and $C_{H}$ are the temperature-dependent heat capacities of the inhibitor and the gas hydrate, respectively $\left[\mathrm{J} \mathrm{kg}^{-1} \mathrm{~K}^{-1}\right]$. 


\subsection{Flux Terms}

The mass fluxes of water, $\mathrm{CH}_{4}$ and inhibitor include contributions from the aqueous and gaseous phases, i.e.,

$$
\mathbf{F}^{\kappa}=\sum_{\kappa \equiv A, G} \mathbf{F}_{\beta}^{\kappa}, \quad \kappa \equiv w, m, i
$$

The contributions of the two solid phases $(\beta \equiv H, I)$ to the fluid fluxes are zero. Therefore, in the kinetic model the mass flux of the hydrate component $(\kappa \equiv h)$ across all subdomain boundaries is

$$
\mathbf{F}^{h}=0
$$

For the aqueous phase, $\mathbf{F}_{A}^{w}=X_{A}^{w} \mathbf{F}_{A}$, and the phase flux $\mathbf{F}_{A}$ is described by Darcy's law as

$$
\mathbf{F}_{A}=-k \frac{k_{r A} \rho_{A}}{\mu_{A}}\left(\nabla P_{A}-\rho_{A} \mathbf{g}\right),
$$

where

$k \quad$ rock intrinsic permeability $\left[\mathrm{m}^{2}\right]$;

$k_{r A}$ relative permeability of the aqueous phase [dimensionless];

$\mu_{A} \quad$ viscosity of the aqueous phase [Pa s];

$P_{A} \quad$ pressure of the aqueous phase $[\mathrm{Pa}] ;$

g gravitational acceleration vector $\left[\mathrm{m} \mathrm{s}^{-2}\right]$.

The aqueous pressure $P_{A}$ is given by

$$
P_{A}=P_{G}+P_{c G W},
$$

where $P_{G}=P_{G}^{m}+P_{G}^{w}$ is the gas pressure $[\mathrm{Pa}], P_{c G W}$ is the gas-water capillary pressure [Pa], and $P_{G}^{m}, P_{G}^{w}$ are the $\mathrm{CH}_{4}$ and water vapor partial pressures [Pa] in the gas phase, 
respectively. The $\mathrm{CH}_{4}$ solubility in the aqueous phase is related to $P_{G}^{m}$ through Henry's law,

$$
P_{G}^{m}=H^{m} X_{A}^{m}
$$

where $H^{m}=H^{m}\left(P_{G}, T\right)[\mathrm{Pa}]$ is a pressure- and temperature-dependent factor akin to Henry's constant. Note that it is possible to determine the $\mathrm{CH}_{4}$ from the equality of fugacities in the aqueous and the gas phase. Although this approach provides a more accurate solution, the difference does not exceed $2-3 \%$ for the vast majority of $\mathrm{CH}_{4}$ hydrate problems in reservoir or laboratory settings, but the execution time can increase as much as $30 \%$.

The mass flux of the gaseous phase $(\beta \equiv G)$ incorporates advection and diffusion contributions, and is given by

$$
\mathbf{F}_{G}^{\kappa}=-k_{0}\left(1+\frac{b}{P_{G}}\right) \frac{k_{r G} \rho_{G}}{\mu_{G}} X_{G}^{\kappa}\left(\nabla P_{G}-\rho_{G} \mathbf{g}\right)+\mathbf{J}_{G}^{\kappa}, \quad \kappa \equiv w, m
$$

where

$k_{0} \quad$ absolute permeability at large gas pressures $(=k)\left[\mathrm{m}^{2}\right]$;

$b \quad$ Klinkenberg [1941] b-factor accounting for gas slippage effects [Pa];

$k_{r G} \quad$ relative permeability of the gaseous phase [dimensionless];

$\mu_{G} \quad$ viscosity of the gaseous phase [Pa s].

The term $\mathbf{J}_{G}^{\kappa}$ is the diffusive mass flux of component $\kappa$ in the gas phase $\left[\mathrm{kg} \mathrm{m}^{-2} \mathrm{~s}^{-1}\right]$, and is described by

$$
\mathbf{J}_{G}^{\kappa}=-\phi S_{G}\left(\phi^{1 / 3} S_{G}^{7 / 3}\right) D_{G}^{\kappa} \rho_{G} \nabla X_{G}^{\kappa}=-\phi S_{G}\left(\tau_{G}\right) D_{G}^{\kappa} \rho_{G} \nabla X_{G}^{\kappa}, \quad \kappa \equiv w, m
$$


where $D_{G}^{\kappa}$ is the multicomponent molecular diffusion coefficient of component $\kappa$ in the gas phase in the absence of a porous medium $\left[\mathrm{m}^{2} \mathrm{~s}^{-1}\right]$, and $\tau_{G}$ is the gas tortuosity [dimensionless] computed from the Millington and Quirk [1961] model. The diffusive mass fluxes of the water vapor and $\mathrm{CH}_{4}$ gas are related through the relationship of Bird et al. [1960]

$$
\mathbf{J}_{G}^{w}+\mathbf{J}_{G}^{m}=0,
$$

which ensures that the total diffusive mass flux of the gas phase is zero with respect to the mass average velocity when summed over the two components $(\kappa \equiv w, m)$. Then the total gas phase mass flux is the product of the Darcy velocity and density of the gas phase.

The flux of the dissolved inhibitor (EQN) is described by

$$
\mathbf{F}_{A}^{i}=X_{A}^{i} \mathbf{F}_{A}+\mathbf{J}_{W}^{i},
$$

where

$$
\mathbf{J}_{W}^{i}=-\phi S_{W}\left(\phi^{1 / 3} S_{A}^{7 / 3}\right) D_{0} \rho_{A} \nabla X_{A}^{i}=-\phi S_{W}\left(\tau_{A}\right) D_{0} \rho_{A} \nabla X_{A}^{i}, \quad \kappa \equiv w, m
$$

$D_{0}$ is the molecular diffusion coefficient of the inhibitor in water, and $\tau_{A}$ is the tortuosity of the aqueous phase.

The heat flux accounts for conduction, advection and radiative heat transfer, and is given by

$$
\mathbf{F}^{\theta}=-\left[(1-\phi) K_{R}+\phi\left(S_{H} K_{H}+S_{I} K_{I}+S_{A} K_{A}+S_{G} K_{G}\right)\right] \nabla T+f_{\sigma} \sigma_{0} \nabla T^{4}+\sum_{\beta \equiv A, G} h_{\beta} \mathbf{F}_{\beta},(2.25)
$$

where

$K_{R} \quad$ thermal conductivity of the rock $\left[\mathrm{W} \mathrm{m}^{-1} \mathrm{~K}^{-1}\right]$;

$K_{\beta} \quad$ thermal conductivity of phase $\beta \equiv A, G, H, I\left[\mathrm{~W} \mathrm{~m}^{-1} \mathrm{~K}^{-1}\right]$; 
$h_{\beta} \quad$ specific enthalpy of phase $\beta \equiv A, G, H, I\left[\mathrm{~J} \mathrm{~kg}^{-1}\right]$;

$f_{\sigma} \quad$ radiance emittance factor [dimensionless];

$\sigma_{0} \quad$ Stefan-Boltzmann constant $\left[5.6687 \times 10^{-8} \mathrm{~J} \mathrm{~m}^{-2} \mathrm{~K}^{-4}\right]$.

The specific enthalpy of the gas phase is computed as

$$
H_{G}=\sum_{\kappa \equiv w, m} X_{G}^{\kappa} h_{G}^{\kappa}+H_{d e p}
$$

where $h_{G}^{\kappa}$ is the specific enthalpy of component $\kappa$ in the gaseous phase, and $H_{d e p}$ is the specific enthalpy departure of the gas mixture $\left[\mathrm{J} \mathrm{kg}^{-1}\right]$. The specific enthalpy of the aqueous phase is estimated from

$$
H_{W}=X_{A}^{w} h_{A}^{w}+X_{A}^{m}\left(h_{A}^{m}+H_{s o l}^{m}\right)+X_{A}^{i}\left(h_{A}^{i}+H_{s o l}^{i}\right)
$$

where $h_{A}^{w}, h_{A}^{m}$ and $h_{A}^{i}$ (see equation (2.14)) are the specific enthalpies of $\mathrm{H}_{2} \mathrm{O}, \mathrm{CH}_{4}$ and the inhibitor at the conditions prevailing in the aqueous phase, respectively, and $H_{s o l}^{m}$ and $H_{\text {sol }}^{i}$ are the specific enthalpy of dissolution $\left[\mathrm{J} \mathrm{kg}^{-1}\right]$ of $\mathrm{CH}_{4}$ and of the inhibitor in the aqueous phase, respectively.

\subsection{Source and Sink Terms}

In sinks with specified mass production rate, withdrawal of the mass component $\kappa$ is described by

$$
\hat{q}^{\kappa}=\sum_{\kappa \equiv A, G} X_{\beta}^{\kappa} q_{\beta}, \quad \kappa \equiv w, m
$$

where $q_{\beta}$ is the production rate of the phase $\beta\left[\mathrm{kg} \mathrm{m}^{-3}\right]$. For a prescribed production rate, the phase flow rates $q_{\beta}$ are determined internally according to different options available 
in $\mathbf{T}+\mathbf{H}$. For source terms (well injection), the addition of a mass component $\kappa$ occurs at desired rates $\hat{q}^{\kappa}(\kappa \equiv w, m)$. Inhibitor injection can occur either as a rate as an individual mass component $\left(\hat{q}^{i}\right)$ or as a fraction of the aqueous phase injection rate, i.e., $\hat{q}^{i}=X_{A}^{i} \hat{q}_{A}$, where $X_{A}^{i}$ is the inhibitor mass fraction in the injection stream.

In the kinetic model, the additional sink/source terms corresponding to hydrate dissociation and release of $\mathrm{CH}_{4}$ and $\mathrm{H}_{2} \mathrm{O}$ must be accounted for. The source term for $\mathrm{CH}_{4}$ thus becomes $\hat{q}^{m}=Q^{m}$, where the production rate $Q^{m}\left[\mathrm{~kg} \mathrm{~m}^{-3} \mathrm{~s}^{-1}\right]$ of $\mathrm{CH}_{4}$ is computed from Equation (2.6) as

$$
Q^{m}=-\frac{W^{m}}{W^{c}} Q_{H},
$$

Similarly, the source term for water (liquid or ice) becomes $\hat{q}^{m}+Q^{m}$, where the hydrate-related release of water $Q^{w}$ is determined from the stoichiometry of Equation $(2.1)$ as

$$
Q^{w}=-\frac{N_{m} W^{w}}{W^{c}} Q_{H},
$$

Under equilibrium conditions, the rate of heat removal or addition includes contributions of (a) the heat associated with fluid removal or addition, as well as (b) direct heat inputs or withdrawals (e.g., microwave heating), and is described by

$$
\hat{q}^{\theta}=q_{d}+\sum_{\kappa=A, G} h_{\beta} q_{\beta}
$$

Under kinetic conditions, the rate of heat removal or addition is determined from

$$
\hat{q}^{\theta}=q_{d}+\sum_{\kappa=A, G} h_{\beta} q_{\beta}+Q_{H} \Delta H^{0},
$$




\subsection{Thermophysical Properties}

\subsubsection{Water}

The properties and parameters of liquid water and steam in $\mathbf{T}+\mathbf{H}$ are provided by (a) fast regression equations based on data from NIST [2000] and (b) steam table equations developed by IFC [1967]. These equations are accurate up to $700{ }^{\circ} \mathrm{C}$ and $100 \mathrm{MPa}$. The code also incorporates additional capabilities extending the temperature and range to $3000{ }^{\circ} \mathrm{C}$ and $1000 \mathrm{MPa}$, but these are based on an iterative approach, are computationally very demanding, and, thus, are not invoked in the current version. The enthalpy, sublimation pressure and fusion/melting pressure of ice (on the ice-vapor and ice-liquid water equilibrium lines of the water phase diagram) are computed using fast regression equations from data obtained using NIST [2000]. Within the solid ice phase (to $T=50 \mathrm{~K}$ and $P \approx 200 \mathrm{MPa}$ ), ice densities were determined using the ice compressibility model of Marion and Jakubowski [2004] and the thermal expansivity data from Dantle [1962]. The ice enthalpy was computed using the heat capacity polynomial equation with the coefficients reported in Yaws [1999].

\subsection{2. $\mathrm{CH}_{4}$-Hydrate}

The hydration number $N_{m}$, and the physical and thermal properties of the $\mathrm{CH}_{4}$-hydrate are input functions of temperature in $\mathbf{T}+\mathbf{H}$. In the determination of $\mathrm{CH}_{4}$-hydrate density as a function of pressure and temperature, the compressibility and thermal expansivity of ice are used in the absence of any data on the subject. The parameters of kinetic dissociation of the hydrate are also inputs to $\mathbf{T}+\mathbf{H}$. The hydration equilibrium pressure- 
temperature relationship and the dependence of the heat of dissociation on temperature are discussed in Section 2.9.

\subsection{3. $\mathrm{CH}_{4} \mathrm{Gas}$}

The properties of the gas phase are provided by the Peng-Robinson equation of state [Peng and Robinson, 1976], one of several options available in the real-gas property package (segment TFx_RealGasEOS.f95, see Section 3.4) included in $\mathbf{T}+\mathbf{H}$. This package computes the compressibility, density, fugacity, specific enthalpy and internal energy (ideal and departure) of pure gases and gas mixtures over a very wide range of pressure and temperature conditions. Additionally, the package computes the gas viscosity and thermal conductivity using the method of Chung et al. [1988], and binary diffusivities from the method of Fuller et al. [1969] and Riazi and Whitson [1993].

The package also allows determination of gas solubility in water by equating fugacities in the gas and aqueous phases, which involves computation of activity coefficients (in the aqueous phase) and fugacities. However, scoping calculations indicated that, for pressures $P<100 \mathrm{MPa}$, accurate estimates can be obtained by alternative correlation relationships included in $\mathbf{T}+\mathbf{H}$. Determination of gas solubility through fugacities and activity coefficients provides accurate estimates for pressures as high as $1000 \mathrm{MPa}$, but is also very computationally demanding, while providing little (if any) benefit for any pressure regime expected in $\mathrm{CH}_{4}$-hydrate studies. Thus, this option is available but deactivated in the current code version. 


\subsection{Hydrate Phase Relationships}

Of particular interest are the pressures and temperatures of the $\mathrm{Lw}-\mathrm{H}-\mathrm{V}$ and $\mathrm{I}-\mathrm{H}-\mathrm{V}$ three-phase lines in the $\mathrm{H}_{2} \mathrm{O}-\mathrm{CH}_{4}$ diagram, which delineate the limits to hydrate formation/dissociation. The relationship between the equilibrium hydration pressure $P_{e}$ and the corresponding equilibrium hydration temperature $T_{e}$ in $\mathbf{T}+\mathbf{H}$ can be obtained from two sources. The first is the regression equation of Kamath [1984]

$$
P=\exp \left(e_{1}+\frac{e_{2}}{T}\right)
$$

where $P$ is in $\mathrm{KPa}, T$ is in $\mathrm{K}$,

$$
e_{1}=\left\{\begin{array}{l}
38.980 \\
14.717
\end{array}, e_{2}=\left\{\begin{array}{ll}
-8533.80 & \text { for } \quad 0^{\circ} \mathrm{C}>T_{c} \geq 25^{\circ} \mathrm{C} \\
-1886.79 & \text { for }-25^{\circ} \mathrm{C} \geq T_{c}>0^{\circ} \mathrm{C}
\end{array} \text { and } T=T_{c}+273.15\right.\right.
$$

The second source is a general regression expression derived by Moridis [2003] based on data from several researchers reported by Sloan [1998]. The two relationships and their range are shown in Figure 2.1. Limited smoothing in the vicinity of the quadruple point $\mathrm{Q}_{1}$ (Figure 2.2) was implemented to allow continuity of the derivatives and smooth phase changes. This is a necessity in the Newton-Raphson iterations implemented in TOUGH+ (see Section 3.4). Because of its limited range, and the discontinuity at the quadruple point, the parametric equations in Figures 2.1 and 2.2 are the default option in $\mathbf{T}+\mathbf{H}$. Use of the Kamath [1984] equation is advised only when phase boundaries are known to not be crossed in the system.

There are limited data on the temperature dependence of heat of dissociation $\Delta H^{0}$, which is often taken as constant over small temperature ranges. In $\mathbf{T}+\mathbf{H}, \Delta H^{0}$ under 
three-phase conditions ( $\mathrm{Lw}-\mathrm{H}-\mathrm{V}$ and $\mathrm{I}-\mathrm{H}-\mathrm{V})$ can be computed from either the simple equation of Kamath [1984] as

$$
\Delta H^{0}=C_{1}+C_{2} T,
$$

where $\Delta H^{0}$ is in cal/gmol, $T$ is in $\mathrm{K}$,

$$
C_{1}=\left\{\begin{array}{l}
13,521 \\
6,534
\end{array} \text {, and } C_{2}=\left\{\begin{array}{l}
-4.02 \text { for } 0{ }^{\circ} \mathrm{C}>T_{c} \geq 25^{\circ} \mathrm{C} \\
-11.97 \text { for }-25^{\circ} \mathrm{C} \geq T_{c}>0{ }^{\circ} \mathrm{C}
\end{array}\right.\right.
$$

Alternatively, it can be determined from the Clausius-Clapeyron equation

$$
\Delta H^{0}=Z R T^{2} \frac{d \ln P}{d T},
$$

using the gas compressibility $Z$ and the known $d(\ln \mathrm{P}) / d T$ of the hydrate equilibrium relationship (from the regression curve, see Figures 2.1 and 2.2). Figure 2.3 shows the uncorrected $\Delta H^{0}$ relationships from both equations. For comparison, Figure 2.3 includes the laboratory measurements of $\Delta H^{0}$ reported in Sloan [1998]. Figure 2.4 shows the modified relationship used in $\mathbf{T}+\mathbf{H}$, which incorporates density-change corrections to the Clausius-Clapeyron equation, as well as smoothing in the immediate vicinity of the quadruple point.

There are no specific measurements of the equilibrium $P-T$ relationship along the I$\mathrm{H}-\mathrm{L}_{w}$ and the I-V-L $\mathrm{L}_{w}$ phase lines of a $\mathrm{H}_{2} \mathrm{O}-\mathrm{CH}_{4} \mathrm{O}$ system, but is generally considered to follow the solidus line (melting/fusion equilibrium) of the water-ice system [NIST, 2000]. Thus, the equilibrium $P$ - $T$ relationship along the I-Lw-H phase line is computed as

$$
P=P_{Q}-6.26 \times 10^{5}\left(1.0-T_{d}^{-3}\right)+1.97135 \times 10^{5}\left(1.0-T_{d}^{21}\right)
$$

where $P$ is in $\mathrm{Pa}, T_{d}=T / 273.16(T$ in $\mathrm{K}), P_{Q}$ (in $\mathrm{Pa}$ ) is the pressure at the hydrate quadruple point (see Figures 2.1 and 2.2). Finally, temperature was considered invariable 
(and equal to $0{ }^{\circ} \mathrm{C}$ along the $\mathrm{I}-\mathrm{V}-\mathrm{L}_{w}$ phase line). The complete phase diagram of the water-- $\mathrm{CH}_{4}-$-hydrate system is shown in Figure 2.4.

\subsection{Inhibitor Effects on Hydrate Equilibrium}

The effect of salinity on the dissociation equilibrium pressure-temperature relationship is described by the equation of Dickens and Quinby-Hunt [1997]

$$
T_{s}=\left[\frac{1}{T_{H}}-\frac{n_{m} \Delta H^{f}}{\Delta H}\left(\frac{1}{273.15}-\frac{1}{T_{f}}\right)\right]^{-1}
$$

In this equation, $T_{s}$ is the equilibrium dissociation temperature in the salt solution $(\mathrm{K})$, $\Delta H^{f}$ is the heat of fusion of ice $(\mathrm{J} / \mathrm{kg}), T_{H}$ is the equilibrium dissociation temperature in the presence of pure water $(\mathrm{K})$, and $T_{f}$ is the freezing point of the salt solution $(\mathrm{K})$. The predictions of this equation are in broad agreement with the measurements of Wright et al. (1999). Further analysis allowed the above complex equation to be replaced with the simpler and equally accurate relationship, which has the additional benefits of applying to both salts and inhibitors such as alcohols. In the simplified equation, the temperature depression (shift) induced by the inhibitors is computed as

$$
\Delta T_{D}=\Delta T_{D, r} \frac{\ln \left(1-x_{A}^{i}\right)}{\ln \left(1-x_{A, r}^{i}\right)},
$$

where

$x_{A}^{i} \quad$ mole fraction of the inhibitor in the aqueous phase;

$x_{A, r}^{i} \quad$ reference mole fraction of the inhibitor in the aqueous phase [K];

$\Delta T_{D} \quad$ inhibitor-induced temperature depression $[\mathrm{K}]$; 
$\Delta T_{D, r}$ temperature depression at the reference mole fraction $x_{A, r}^{i}$.

This approach is entirely consistent with the equation of Makogon [1981] for alcohols. Exploratory calculations have shown this equation to be within $3-5 \%$ of the estimates of the far more computationally intensive method of Dickens and Quinby-Hunt [1997].

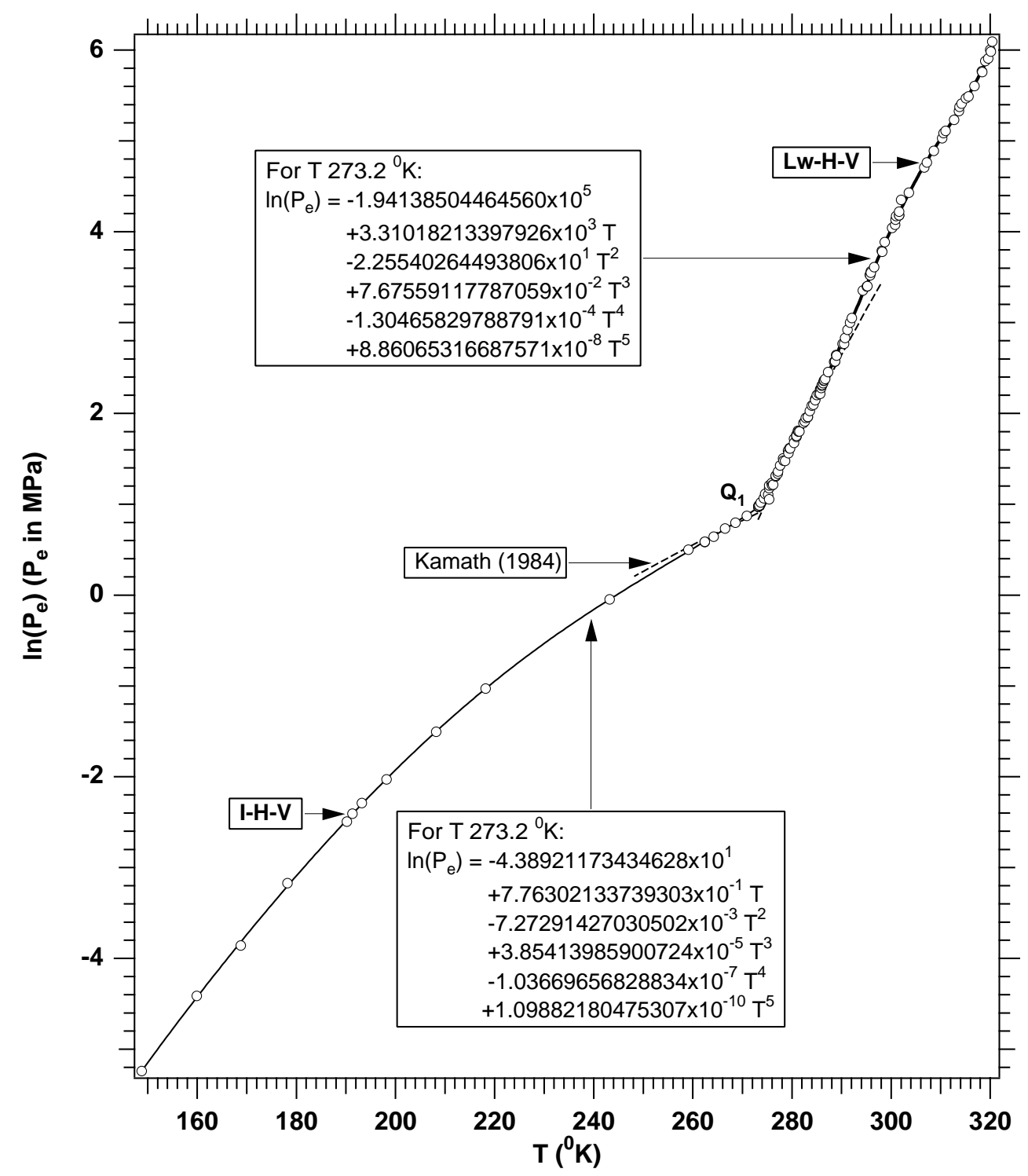

Figure 2.1. Relationship of the equilibrium hydration pressure $\mathrm{Pe}$ and temperature $\mathrm{Te}$ of the $\mathrm{CH}_{4-}$ hydrate [Moridis, 2003]. 


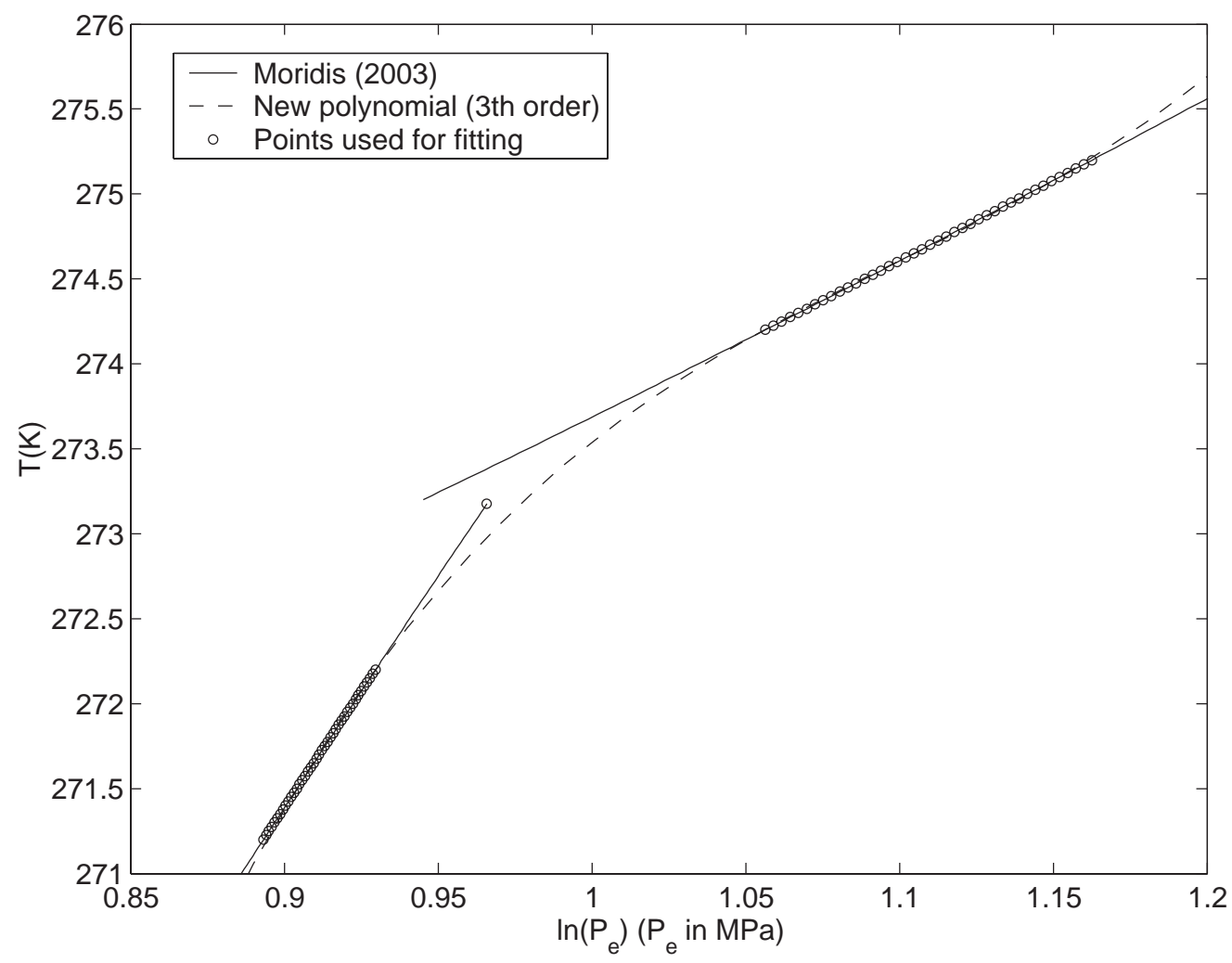

Figure 2.2. Modified $P e-T e$ relationship in the vicinity of the quadruple point. 

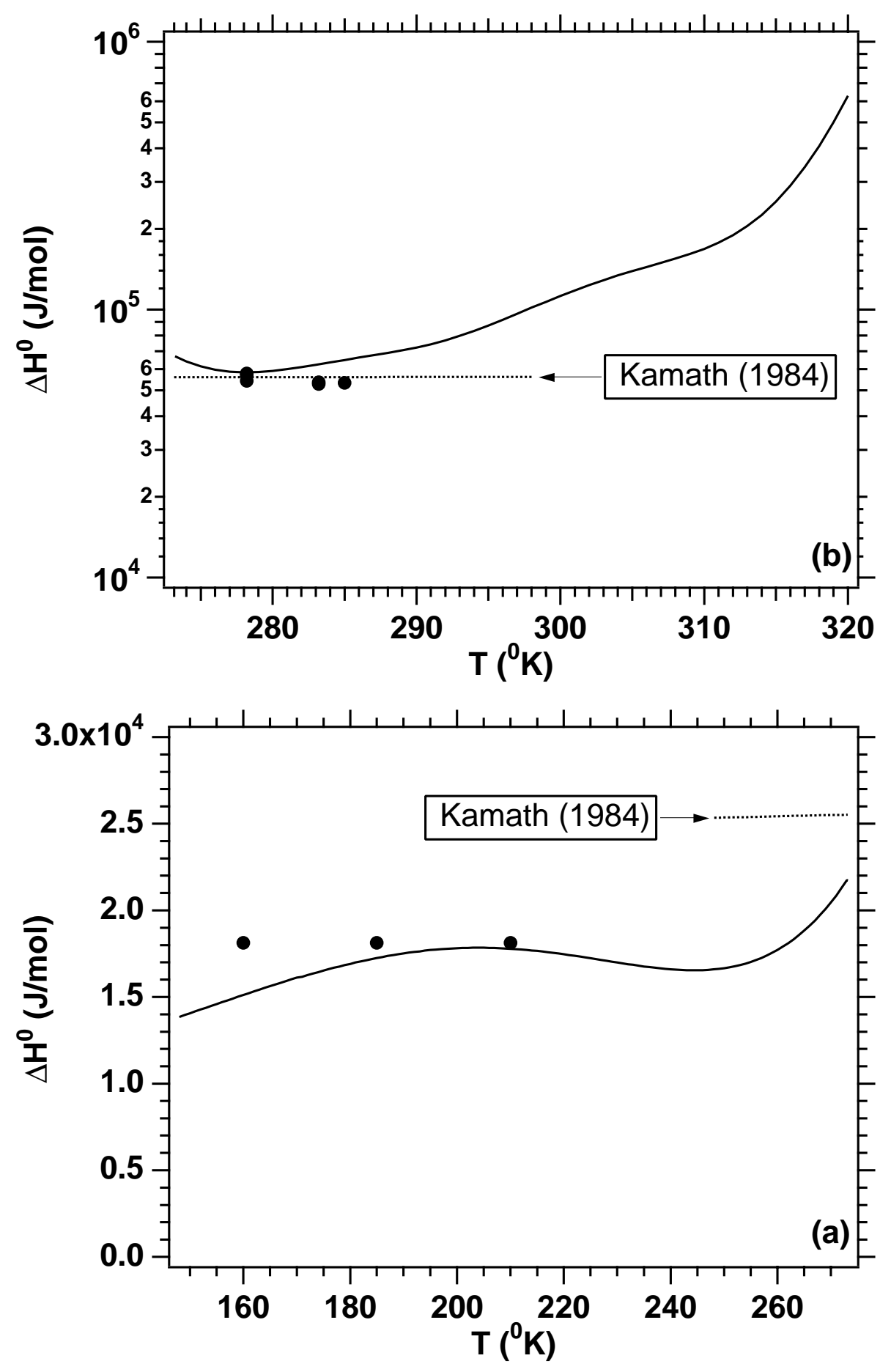

Figure 2.3. Temperature dependence of the $\mathrm{CH}_{4}$-hydrate heat of dissociation $\Delta H^{0}$ along the (a) I$\mathrm{H}-\mathrm{V}$ and (b) $\mathrm{L}_{w}-\mathrm{H}-\mathrm{V}$ phase lines [Moridis, 2003]. 


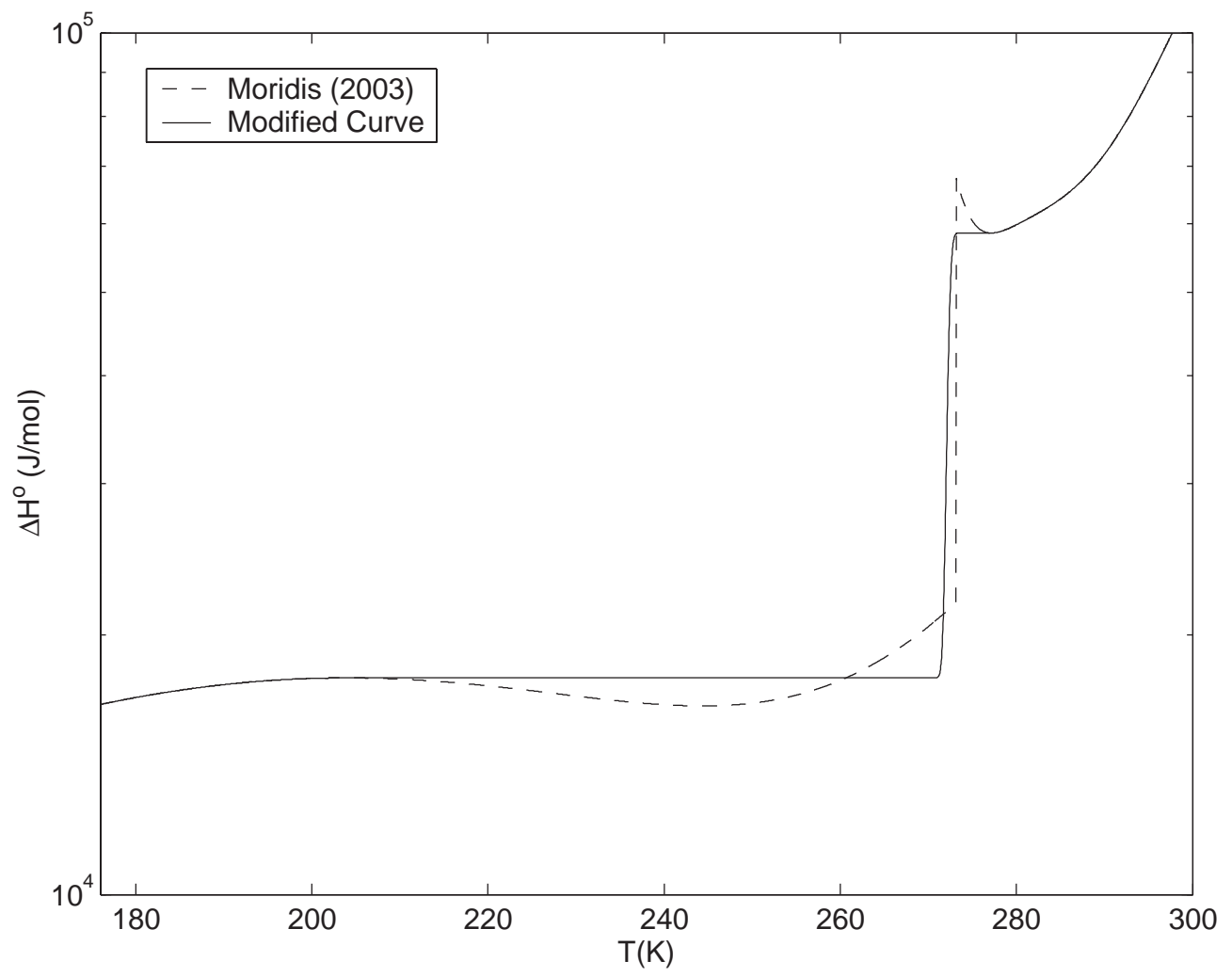

Figure 2.4. Modified $\Delta H^{0}$ vs. Te relationship used in TOUGH+HYDRATE. 


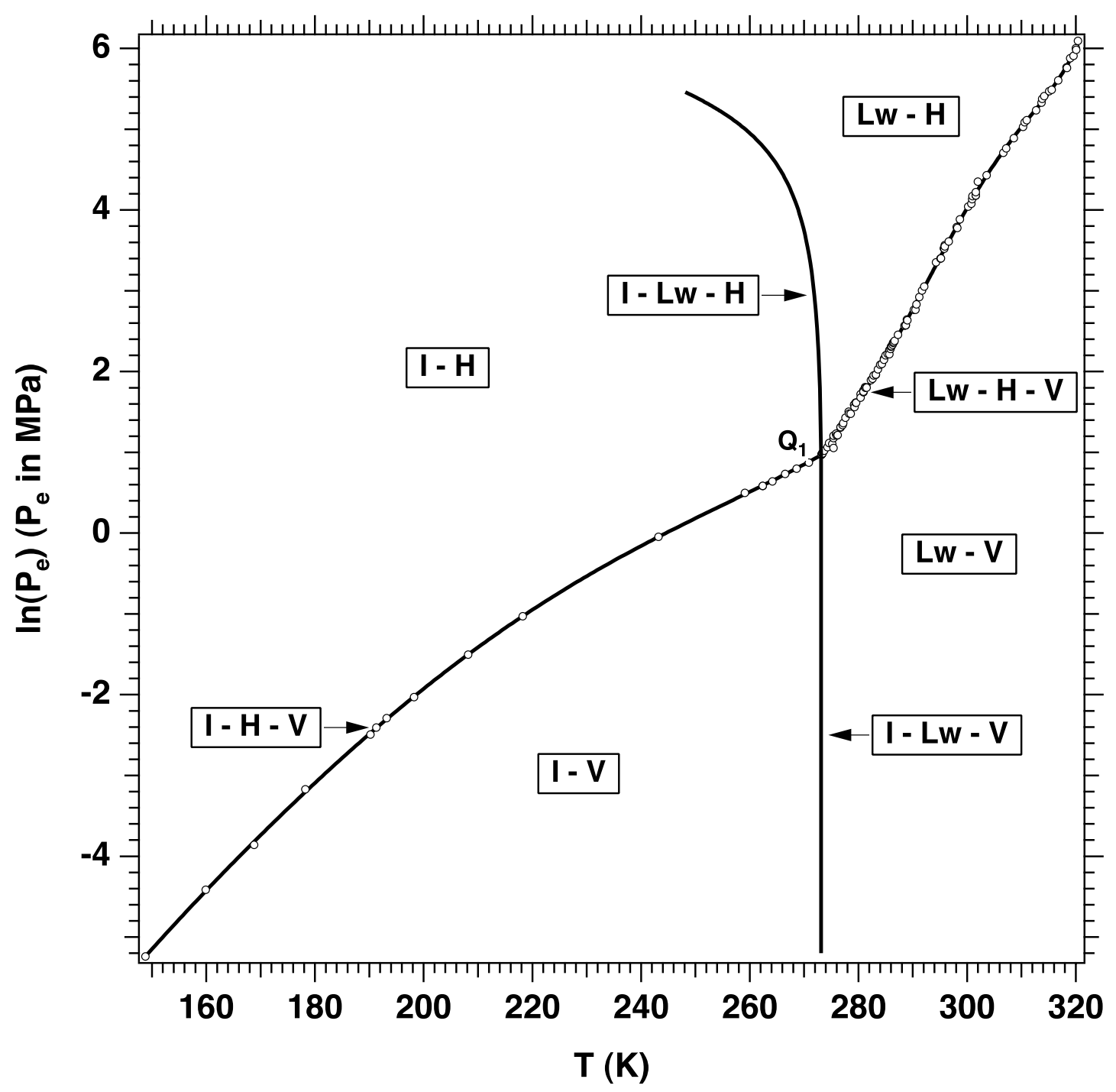

Figure 2.5. Pressure-temperature equilibrium relationship in the phase diagram of the water-$\mathrm{CH} 4--$ hydrate system in $\mathrm{T}+\mathrm{H}$. 


\subsection{Porosity and intrinsic permeability change}

When solid phases are deposited in a porous medium, through chemical precipitation or freezing of pore fluids, the ability of the porous medium to transmit fluids can change profoundly. The deposition of solids in a porous medium reduces the void space available for fluids. Such reduction in porosity will give rise to a reduction in permeability as well. There is an extensive literature, going back to the 1920 s, about the manner in which permeability declines as portions of the pore space are filled by solids, and a bewildering variety of porosity-permeability correlations have been obtained from experimental and theoretical studies [Scheidegger, 1974, and references therein; Morrow et al., 1981; Vaughan, 1987; Verma and Pruess, 1988; Phillips, 1991; Pape et al., 1999; $X u$ et al., 2004]. Within the scope of the work undertaken here it is not possible to perform a thorough review of different permeability reduction models, and to evaluate their suitability for representing permeability reduction due to formation of hydrate and/or ice. Dearth of relevant information prevents considering whether any of the models developed for solid precipitation in porous media are valid to gas/water/ice/hydrate systems. Instead, we briefly discuss salient features of pore channels to highlight the most important effects, and then explain the rationale behind the preliminary choices made in this study.

It is obvious that permeability effects depend not just on the magnitude of porosity change, but on geometric properties of the pore channels, and on where and how solid deposition in those channels occurs. The lack of unanimity among different investigators about the correlation between porosity and permeability change reflects the great diversity of pore channel geometries and precipitation processes in porous media. The 
simplest models conceptualize porous media as bundles of capillary tubes, which gives rise to a simple power law dependence of permeability $k$ on porosity $\phi$,

$$
\frac{k}{k_{0}}=F_{\phi S}=\left(\frac{\phi}{\phi_{0}}\right)^{n}
$$

where the term $F_{\phi S}$ is a permeability adjustment factor that describes the effects of (a) the presence of solid phases other than the medium grains (such as ice, hydrate or precipitating salts), and (b) changes in porosity on permeability, and the subscript " 00 " denotes properties at a reference state.

The exponent $n$ typically will be in the range from 2 to 3 [Phillips, 1991], giving a rather mild dependence of permeability on porosity. However, in media with intergranular porosity, pore channels generally have a convergent-divergent geometry, consisting of a succession of 'pore throats' with small radius and 'pore bodies' with large radius (Figure 2.6).

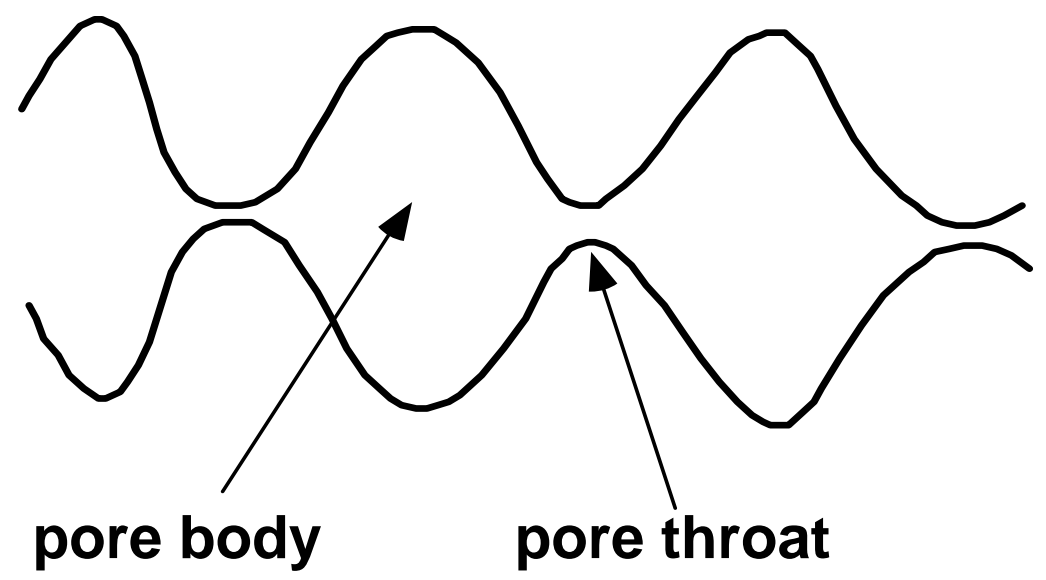

Figure 2.6. Schematic of pore channels, showing convergent-divergent geometry with a succession of pore throats and pore bodies. 
If solids are deposited uniformly along the pore walls, or are preferentially deposited in the throats, then even relatively minor amounts of deposition can give rise to a dramatic decrease in permeability. Such behavior has been observed in field and laboratory-scale systems, including the diagenesis of sandstones [Pape et al., 1999], precipitation around geothermal injection wells [Xu et al., 2004], and hydrothermal flows in laboratory specimen [Morrow et al., 1981; Vaughan, 1987].

In these systems, a rather modest amount of precipitate, that leaves most of the original pore space available for fluids, nonetheless caused order-of-magnitude changes in absolute permeability. Such behavior can be understood from 'tubes-in-series' models of pore space, as shown in Figure 2.7 [Verma and Pruess, 1988]. If one assumes that solids are deposited as a layer of uniform thickness on the pore walls, then permeability will be reduced to zero when the throats become clogged, while plenty of (disconnected) porosity remains in the pore bodies. This leads to the concept of a non-zero 'critical porosity' $\phi_{c}$ at which permeability is reduced to zero, with a permeability reduction as given in Equation (2.41) [Verma and Pruess, 1988; Xu et al., 2004].

$$
\frac{k}{k_{0}}=F_{\phi S}=\left(\frac{\phi-\phi_{c}}{\phi_{0}-\phi_{c}}\right)^{n}
$$

Fractal models also give a very strong dependence of permeability on porosity, with exponents in relationships such as Equation (2.41) as large as $n=10$ or more [Pape et al., 1999]. The above discussion clearly indicates the need fundamental research for the determination of the sites within the porous media at which hydrates and ice form preferentially. 


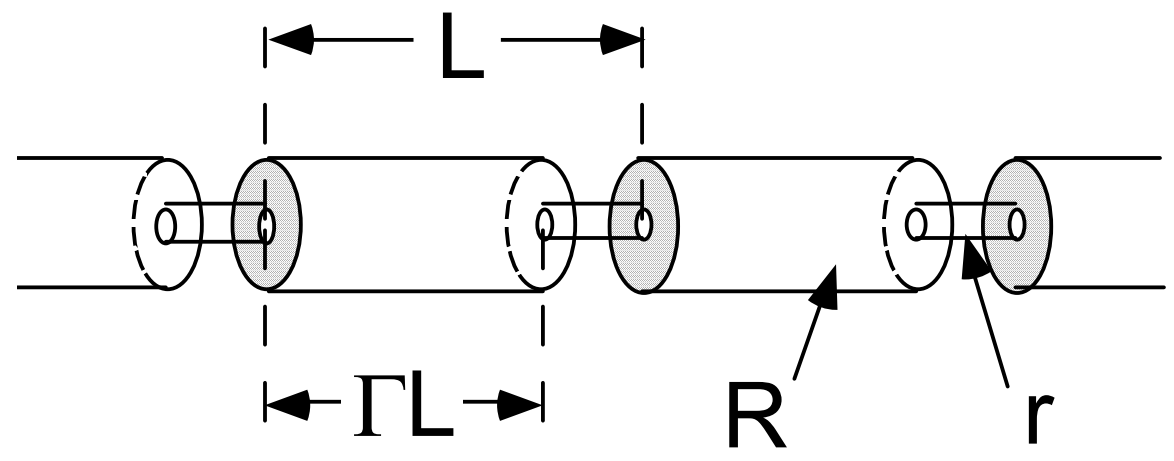

Figure 2.7. Tubes-in-series model of pore channels.

\subsection{Wettability Phenomena}

\subsubsection{Capillary pressure}

Effects of solids deposition become considerably more complicated, and involve more than just permeability change, when multiple fluid phase are present, such as water and gas. Surface tension effects between different phases give rise to 'capillary pressures', denoted by $P_{c a p}$, which may be profoundly altered when solids are deposited. Most mineral surfaces are preferentially wetted by water and, under partially saturated conditions, the pressure of the wetting (aqueous) phase inside a porous medium will be less than that of the non-wetting (gas) phase. The pressure difference $P_{c a p}=P_{g}-P_{l}$ is termed capillary pressure, because it relates to the phenomenon of water level rise in a capillary tube. (Sometimes $P_{c a p}$ is defined with the opposite sign.)

Issues relating to capillary pressure can be conveniently discussed with reference to the pore size distribution of the porous medium. Figure 2.8 shows a schematic probability density function (pdf) for pore sizes, which expresses the probability $p(r)$ of having pores with radius $r$. At a given capillary pressure $P_{c a p}$, pores with radius $r^{\prime} \leq r$ 
may be water-filled, where the cutoff radius $r$ is related to the capillary pressure by the Young-Laplace equation (reference)

$$
P_{c a p}=\frac{2 \sigma \cos (\gamma)}{r}
$$

Here, $\sigma$ is the surface tension (energy per unit surface area) at the water-gas interface, and $\gamma$ is the contact angle, which usually is close to zero for preferentially water-wet minerals.

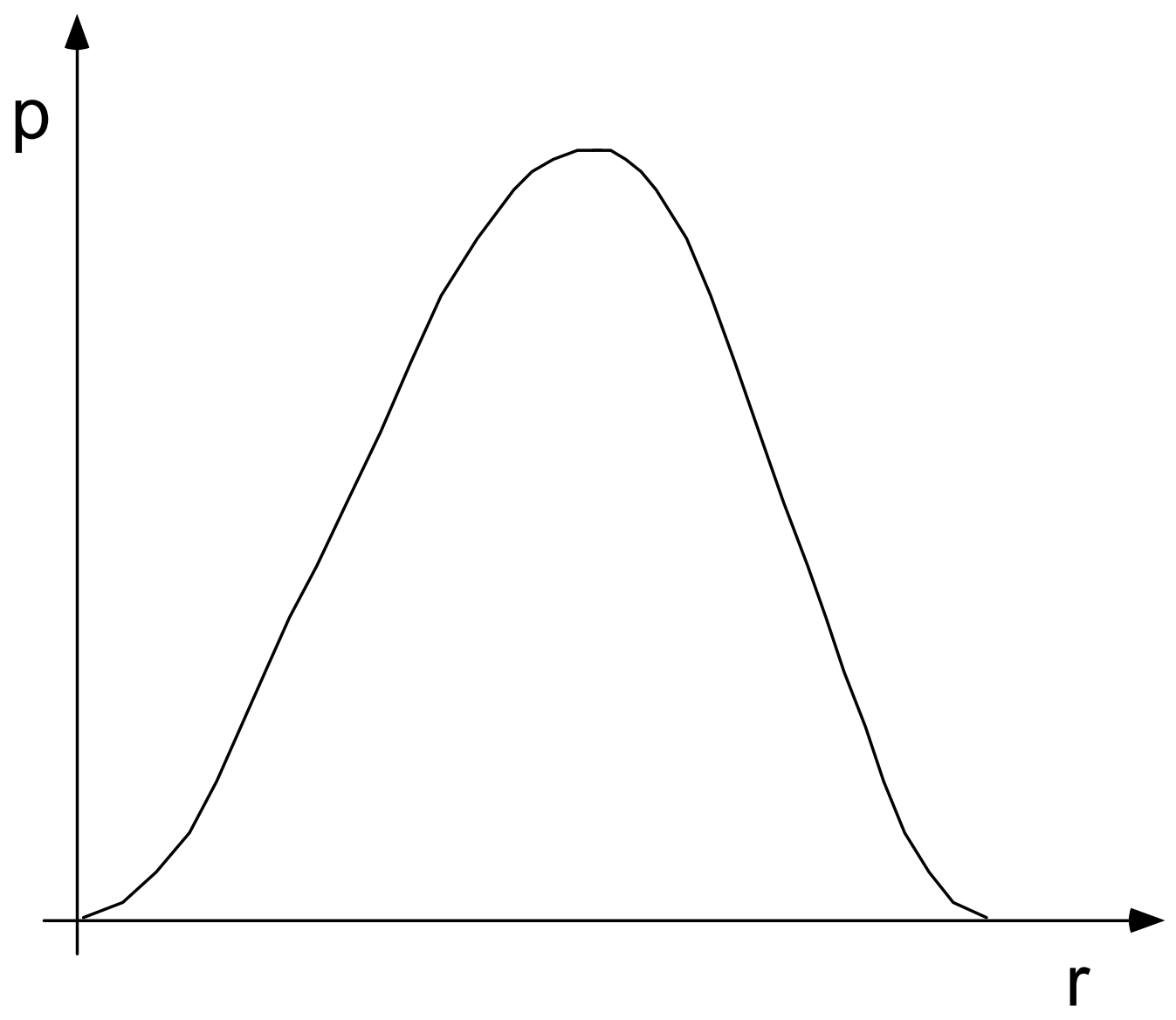

Figure 2.8. Schematic of probability density function $p(r)$ for pore size distribution.

In introducing Equation (2.41) we stated that pores with $r^{\prime} \leq \mathrm{r}$ may be water filled, but whether indeed all pores with $r^{\prime} \leq r$ will be water-filled at a prevailing capillary pressure 


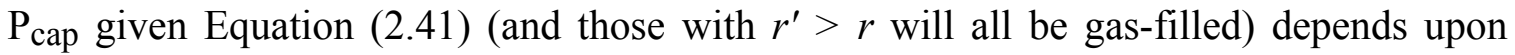
issues of pore accessibility that are not captured by the pore size distribution. The issue of accessibility arises because pores of certain radius may be entirely surrounded by larger pores, or by smaller pores, so that either water may not enter them during a wetting process, or may not be removed from them during a draining process. Accessibility gives rise to the well-known phenomenon of capillary hysteresis, where at a given magnitude of capillary pressure, water saturation will generally be larger during a drainage process than during a wetting process [de Marsily, 1986]. Although capillary hysteresis is a wellestablished effect, it is seldom taken into account in modeling applications; this is partially due to numerical difficulties associated with it, partially because information on applicable parameters is rarely available.

Formation of solid phases will alter the pore size distribution, generally reducing pore sizes, and thereby giving rise to stronger capillary pressures (see Equation 2.41). In order to estimate these changes, we require information on the original pore size distribution of the medium, and on the manner in which the pore size distributions will be altered during solids deposition. As no such information is presently available for the medium studied here, we proceed in a more phenomenological manner, and relate changes in capillary pressures to overall changes in porosity and permeability of the medium. Examining a variety of unconsolidated media, Leverett [1941] determined a dependence of capillary pressure on permeability and porosity, as follows.

$$
P_{c a p}\left(S_{A}\right)=\sqrt{\frac{k_{00}}{k} \cdot \frac{\phi}{\phi_{00}}} P_{c a p, 00}
$$


where $P_{c a p, 00}$ is the capillary pressure corresponding to a reference medium at the reference conditions, at which the permeability and porosity of the porous medium are $k_{00}$ and $\phi_{00}$, respectively. Equation (2.44) is used in the present analysis, in the following manner. We represent active solids (i.e. ice that may melt or hydrate that may dissociate, as opposed to solid minerals which are inert) by means of a solid saturation, denoted by $S_{S}=S_{H}+S_{I}$, which measures the fraction of active pore space occupied by solids. The fraction of pore space available to fluid phases is $S_{A}+S_{G}$, and we have the constraint

$$
S_{A}+S_{G}=1-S_{S}
$$

Let $P_{c a p, 00}$ denote the capillary pressure function applicable to a porous medium free of solid saturation $\left(S_{S}=0\right)$ with a reference porosity $\phi_{00}$. The total current porosity $\phi$ and the active (or available) porosity $\phi$ available to fluids are then defined by the equation

$$
\phi_{a}=\underbrace{\left(F_{P T} \phi_{r r} \phi_{00}\right)}_{\phi}\left(S_{A}+S_{G}\right) \Rightarrow \frac{\phi_{a}}{\phi_{00}}=F_{P T} \phi_{r r}\left(S_{A}+S_{G}\right)=F_{P T} \phi_{r r}\left(1-S_{S}\right),
$$

where the term $F_{P T}$ is a porosity adjustment factor that accounts for the effects of pressure and temperature on porosity, and $\phi_{r}$ is the $\phi$ relative magnitude, which relates the porosity $\phi_{0}$ of a given medium to the porosity $\phi_{00}$ of the reference medium at the same reference $P$ and $T$. This term is introduced to account for situations in which the reference medium is different from the one under consideration, as is often the case when insufficient data are available and parameter estimation is based on scaling using known media as references. For a reference medium different from the one under consideration, $\phi_{r}=\phi_{0} / \phi_{00}$. It is obvious that $\phi_{r}=1$ when the same medium is used as reference.

When changes in $P$ and $T$ are not large in geomechanically stable media, then $F_{P T}$ can be estimated from the following equation: 


$$
F_{P T}=\frac{\phi}{\phi_{r r} \phi_{00}}=\frac{\phi}{\phi_{0}}=\exp \left[\alpha_{P} \Delta P+\alpha_{T} \Delta T\right] \approx 1+\alpha_{P} \Delta P+\alpha_{T} \Delta T,
$$

where $\Delta P=P-P_{0}, \Delta T=T-T_{0}, \alpha_{P}$ and $\alpha_{T}$ are the pore compressibility and thermal expansivity, respectively (see discussion in Section 6.2). For large $\Delta P$ and/or $\Delta T$ in compressible or geomechanically unstable media, $F_{P T}$ can be estimated from a full geomechanical model that relates the resulting changes in geomechanical stresses and strains to changes in porosity.

The argument in the capillary pressure function $P_{c a p, 0}$ on the r.h.s of Equation (2.44) is the aqueous saturation $S_{A}$, referred to total fluid porosity. We measure liquid saturation on a scale that refers to total active (fluid plus hydrate- and ice-filled) pore space in the ice- and hydrate-free porous medium. In the medium with solid saturation $S_{S}, S_{A}$ corresponds to a scaled saturation

$$
S_{A}^{*}=\frac{S_{A}}{S_{A}+S_{G}}
$$

relative to fluid-filled pore space, and this is the value to be used in the estimation of $P_{c a p, 0}$ in the r.h.s of Equation (2.44). In the next session we will discuss the estimation of $k_{r \phi}$ in the presence of solid phases (i.e., ice and/or hydrate) for use in Equation (2.44).

\subsubsection{Relative Permeability}

In multiphase flow, each fluid phase occupies only part of the pore space, and its effective permeability is reduced due to interference with the other phase(s). This effect is represented by means of permeability reduction factors or relative permeabilities, customarily denoted by $k_{r A}$ and $k_{r G}$ for liquid (aqueous) and gas, respectively, such that effective permeability $k_{\beta}$ to phase $\beta(=\mathrm{A}, \mathrm{G})$ is given by 


$$
k_{\beta}=k k_{r \beta} \text {, where } k=k_{0} F_{\phi S}=k_{00} k_{r r} F_{\phi S} \text {, }
$$

and $k_{r r}$ is the $k$ relative magnitude that relates the permeability $k_{0}$ of a given medium to $k_{00}$ of the reference medium at the same $P$ and $T$. As in the case of $\phi_{r r}, k_{r r}$ is introduced to account for situations in which the reference medium is different from the one under consideration, as is often the case when insufficient data are available and parameter estimation is based on scaling such as the one described by Equation (2.44) using known media as references. For a reference medium different from the one under consideration, $k_{r}=k_{0} / k_{00}$. It is obvious that $k_{r}=1$ when the same medium is used as reference.

The relative permeabilities are functions of the phase saturations $S_{\beta}$ (fraction of pore space occupied by phase $\beta), k_{r \beta}=k_{r \beta}\left(S_{\beta}\right)$, and are usually obtained by measurement on laboratory specimen of porous media. Figure $\mathbf{2 . 9}$ gives examples of commonly used liquid and gas relative permeabilities.

The partitioning of effective permeability to a fluid phase $\beta$ into a porous mediumand solid-saturation dependent part $\left(k_{0} F_{\phi S}\right)$ and a fluid saturation-dependent part $k_{r \beta}$ is a matter of convention and convenience. It leads to a conceptual ambiguity in the representation of permeability reduction from solid deposition in multiphase flow. Indeed, such permeability reduction may be attributed either to a change in absolute or intrinsic permeability (as described by the product $k_{0} F_{\phi S}$ ), as is done for single-phase flow, or it may be attributed to a change in the fluid relative permeability $k_{r} \beta$.

When hydrate and/or ice forms inside a partially water-saturated porous medium, such formation clearly must start in the water-filled portion of the pore space, but may not remain limited to the water-filled portion, as the solid crystals may grow and extrude into primarily gas-filled pores. In the absence of specific pore-scale information about 
where hydrate and/or ice will likely form, it is not possible to ascertain the applicability of relationships such as Equation (2.41) to the permeability reduction associated with hydrate formation and/or freezing. Even if applicable, appropriate parameters for the problem under study here are lacking.

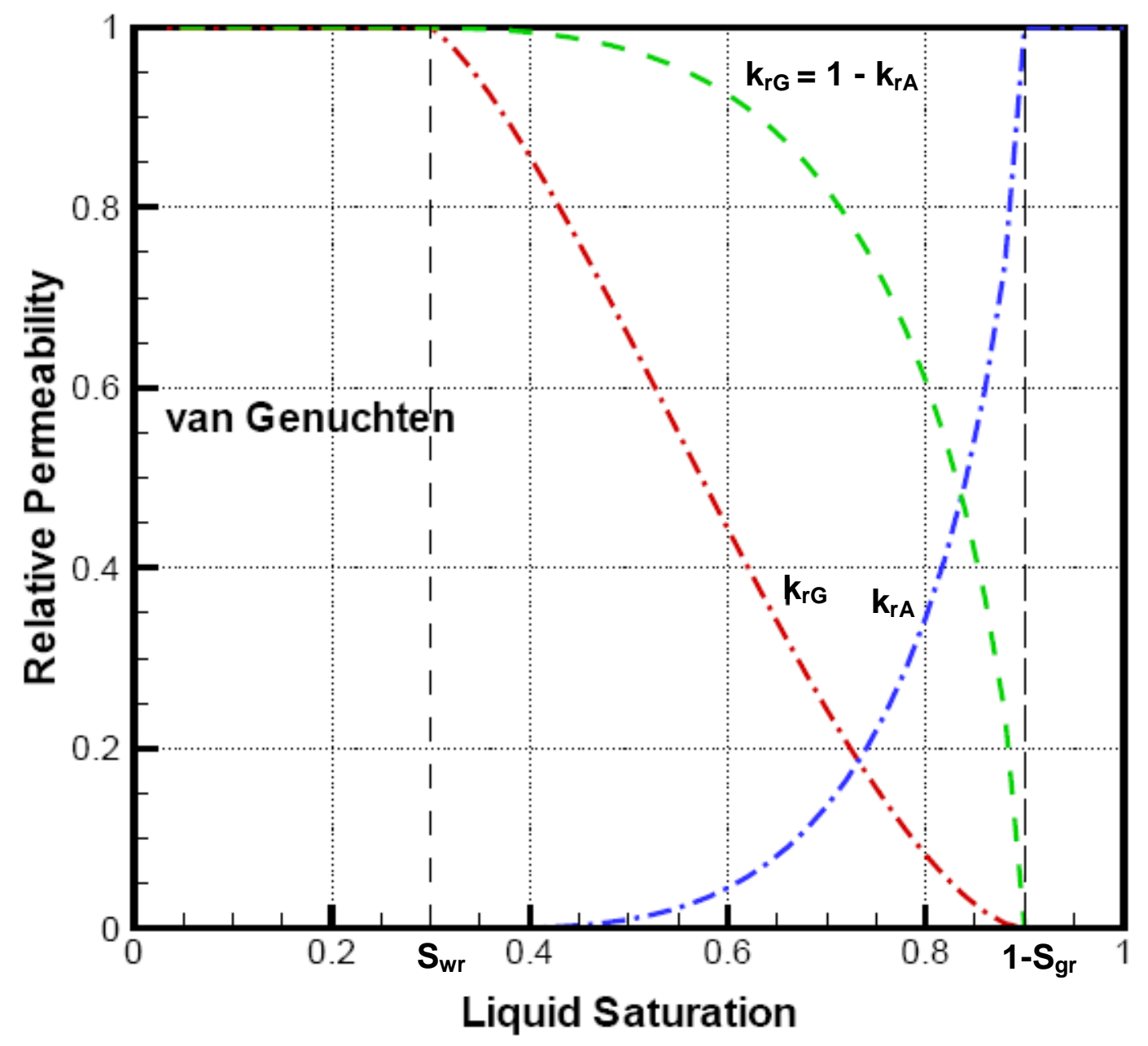

Figure 2.9. Liquid and gas relative permeabilities based on the van Genuchten model [Finsterle, 1999]. 
We therefore propose two alternative models to describe the wettability processes (relative permeability and capillary pressure) in hydrate- and/or ice-bearing media. The first model, hereafter referred to as the "Original Porous Medium" (OPM) model, is based on the treatment of (a) the medium porosity as unaffected by the emergence of hydrates and/or ice (although subject to change due to changes in pressure and temperature), (b) of the intrinsic permeability of the porous media as unchanging during the evolution of the solid phases, and (c) of the fluid flow as a relative permeability issue controlled by the saturations of the various phases in the pores. The second family of models, hereafter referred to as the "Evolving Porous Medium" (EPM) models, consider the evolution of the solid phases (hydrate and ice) as tantamount to the creation of a new porous medium with continuously changing porosity and intrinsic permeability, the pore space of which is occupied only by the two fluid phases (aqueous and gas).

2.12.2.1. The OPM model. This simpler model represents permeability reduction as relative permeability effects, and does not require any new parameters to be introduced. More specifically, this model assumes that in the presence of solid phase(s), relative permeability to each fluid phase is given by the same function $k_{r}\left(S_{\beta}\right)$ as in the absence of solids. This means that aqueous phase relative permeability $k_{r A}=k_{r A}\left(S_{A}\right)$ depends only on the aqueous saturation $S_{A}$, and is the same, regardless of how the remaining fraction $\left(1-S_{A}\right)$ of the pore space is divided between gas and solid phases. A similar comment applies to gas relative permeability $k_{r G}=k_{r G}\left(S_{G}\right)$.

Setting aside for a moment the issue of $\left(k_{r \phi} k_{r S}\right)$, permeability reduction for the fluid phases then occurs simply because, when $S_{S}$ increases, fluid phase saturations $S_{A}$ and $S_{G}$ 
generally must decrease also, as dictated by the constraint in Equation (2.43). This prescription is tantamount to asserting that liquid phase flow behaves as though solids deposition occurs entirely in what would otherwise be gas-filled pore space, while gas phase flow behaves as though solids deposition occurs entirely in what otherwise would be liquid-filled pore spaces. It is obvious that solids deposition cannot simultaneously occur only in liquid and only in gas-filled pore spaces, which points to a limitation of the proposed permeability reduction model. We nonetheless feel that a model that introduces no new and uncertain parameters is preferable to a model that does.

The permeability adjustment factor is computed from the following expression:

$$
F_{\phi S}=k_{r \phi} k_{r S},
$$

where $k_{r \phi}$ is the permeability $\phi$-factor that describes the effect of changes in $\phi$ on permeability, and $k_{r S}$ is the permeability $S$-factor that relates reduction in the intrinsic permeability to the presence of solid phases (such as ice, hydrates or precipitating salts).

In the OPM model, $k_{r S}=1$ by definition, and the permeability $\phi$-factor in Equation (2.49) can be computed as

$$
k_{r \phi}=\left\{\begin{array}{l}
1 \quad \text { when the effect of } \phi \text { changes on } k \text { is neglected } \\
\exp \left[\gamma\left(F_{P T}-1\right)\right] \text { when the effect of } \phi \text { changes on } k \text { is accounted for, }
\end{array}\right.
$$

where $\gamma$ is an empirical parameter [Rutqvist and Tsang, 2002], and $F_{P T}$ is computed from Equation (2.47).

In the OPM model, capillary pressures are estimated from Equation (2.44), in which:

- $\phi / \phi_{00}=\phi_{r r} F_{P T}$ is computed from Equation (2.47) 
- $k_{00} / k=1 / k_{r r} k_{r \phi}$ is computed from Equations (2.49) and (2.50), and

- $P_{c a p, 00}$ is computed based on the scaled saturations $S^{*}$ of Equation (2.48)

Thus, the final expression for estimating the capillary pressure in the OPM model is:

$$
P_{c a p}=\sqrt{\frac{\phi_{r r}}{k_{r r}} \cdot \frac{F_{P T}}{k_{r \phi}}} \cdot P_{c a p, 00}\left(S^{*}\right)
$$

Additional scaling can be introduced by using the active porosity $\phi_{a}$ and $\phi_{a} / \phi_{00}$ from Equation (2.46) - as opposed to $\phi / \phi_{00}$ from Equation (2.47) - in the computation of Equation (2.44).

2.12.2.2. The EPM models. In recognition of, and attempting to overcome, the limitations of the OPM permeability reduction model, we have proposed two EPM models and performed sensitivity studies using the absolute (intrinsic) permeability modifications that will be discussed in this section. While the EPM models provide valuable insights, a more consistent and defensible model - based on both theoretical analyses and laboratory and field studies - for the effects of emerging solid phases on fluid permeabilities should be developed in the future.

EPM Model \#1. With intrinsic permeability modification based on relative permeabilities, what absolute permeability should be used in the Leverett scaling Equation (2.41)? An attractive possibility would be to set $k=k_{0}\left(k_{r A}+k_{r G}\right)$. This, however, is not acceptable because the sum of liquid and gas relative permeabilities depends not just on the solid saturation $\left(S_{S}\right)$, but on $S_{A}$ and $S_{G}$ individually.

As a plausible alternative, we consider the permeability reduction when the fluidavailable pore space is either entirely liquid-filled or entirely gas-filled, which leads to 


$$
k_{r S}=k_{r A}\left(S_{A}=1-S_{S}\right) \quad \text { or } \quad k_{r S}=k_{r G}\left(S_{G}=1-S_{S}\right)
$$

Note that either expression depends only on solid saturation $S_{S}$. As an estimate of the $k_{r S}$, we then take the average of the two,

$$
k_{r S}=\frac{1}{2}\left[k_{r A}\left(S_{A}=1-S_{S}\right)+k_{r G}\left(S_{G}=1-S_{S}\right)\right]
$$

Equation (2.50) provides a simple estimate of the permeability $\phi$-factor. Then the phase effective permeabilities are computed using Equation (2.49), in which:

- $k_{r \beta}$ is computed based on the scaled saturations from Equation (2.48),

- $F_{\phi S}$ is computed from Equation (2.50),

- $k_{r \phi}$ is computed from Equation (2.51), and

- $k_{r S}$ is computed from Equation (2.53).

The capillary pressure in the EPM \#1 model is estimated using Equation (2.44), in which the various terms are computed as follows:

- $\phi_{a} / \phi_{00}$, computed from Equation (2.46), is used instead of $\phi / \phi_{00}$,

- $k_{00} / k=1 / k_{r r} F_{\phi S}$ is computed from Equations (2.49) and (2.50),

- $k_{r \phi}$ is computed from Equation (2.51), and

- $k_{r S}$ is computed from Equation (2.53).

Thus, the final expression for estimating the capillary pressure in the EPM \#1 model is:

$$
P_{c a p}=\sqrt{\frac{\phi_{r r}}{k_{r r}} \cdot \frac{F_{P T}\left(1-S_{S}\right)}{k_{r \phi} k_{r S}}} \cdot P_{c a p, 00}\left(S^{*}\right)
$$

EPM Model \#2. The only difference between this model and the EPM \#1 model is in the equation used to estimate the $k_{r S}$ term, with all other equations applying unchanged. 
In the EPM\#2 model, the quantity $F_{\phi S}=k_{r \phi} k_{r S}$ in Equation (2.50) is provided by Equation (2.42), leading to

$$
k_{r S}=\left[\frac{\phi_{0}\left(1-S_{S}\right)-\phi_{c}}{\phi_{0}-\phi_{c}}\right]^{n}
$$

The term $k_{r \phi}$ is obtained from Equation (2.51). Thus, the fluid effective permeabilities in the EPM \#2 model are computed from Equation (2.49). Similarly, the capillary pressure in the EPM \#2 model is estimated using Equation (2.54), using $k_{r S}$, from Equation (2.54).

\subsubsection{Pore compressibility of unconsolidated media in the}

\section{presence of cementing solid phases}

While the pore compressibility $\alpha_{P}$ in Equation (2.47) can be considered as a constant or even as a function of pressure during fluid flow through consolidated (lithified) porous media and/or in unconsolidated media, this approach is inadequate when cementing solid phases (such as ice and/or hydrates) are present in the pores. This is because the presence of these solid phases imparts stiffness and increases the geomechanical strength of the solid phase-impregnated porous medium, the porosity $\phi$ of which reacts much slower to variations in pressure $P$. Thus, an accurate representation of the evolution of $\phi$ as a function of $P$ in these cases needs to account for the effect of the saturation $S_{S}$ of such solid phases.

The most appropriate method for accounting for the effect of $S_{S}$ on the porosity of unconsolidated media is by solving the coupled flow-geomechanical problem, estimating variations in $P, T$ and phase saturation, and computing the corresponding changes in 
stresses and strains. These are then used to compute changes in $\phi$ and $k$. Such coupling is a possibility in the TOUGH+ code, which allows the use of the commercial geomechanical model FLAC3D (ITASCA, 2006) to evaluate the interaction between flow and geomechanical properties. This model is automatically invoked if the corresponding executable file FLAC3D.exe is present in the TOUGH+ directory and appropriate inputs are provided to the TOUGH+ code (see detailed discussion in Section 5).

If the FLAC3D model is not available or is not invoked (a frequent choice, given the large execution times required for such fully coupled flow-geomechanical problems), it is possibly to describe the effect of cementing solid phases $S_{S}$ on the porosity $\phi$ and the intrinsic permeability $k$ of unconsolidated media by employing an empirical model that describes the media compressibility as:

$$
\alpha_{P}=\exp \left\{\ln \alpha_{P L}+\left(\ln \alpha_{P U}-\ln \alpha_{P L}\right)\left[1-B_{x}\left(2.25,2.25, S_{S}^{*}\right)\right]\right\}
$$

where

$$
S_{S}^{*}=\frac{S_{S}-S_{S \min }+\delta}{S_{S \max }-S_{S \min }+2 \delta}
$$

$\alpha_{P L}$ is the lower limit of the medium compressibility (corresponding to the full stiffening/strengthening effect of the presence of cementing solid phases such as ice and/or hydrates), $\alpha_{P U}$ is the upper limit of the medium compressibility (corresponding to the absence of cementing solid phases), $B_{x}$ is the incomplete beta function, $S_{\text {Smin }}$ is the largest $S_{S}$ saturation at which $\alpha_{P}=\alpha_{P U}, S_{S \max }$ is the lowest $S_{S}$ saturation at which $\alpha_{P}=$ $\alpha_{P L}$, and $\delta$ is a smoothing factor. Equation (2.56) is based on geomechanical and geophysical data derived from laboratory and field observations, and results in the curve of Figure 2.10 that scans between the $\alpha_{P U}$ and the $\alpha_{P L}$ compressibility limits. 
The relative porosity $\phi / \phi_{0}$ is estimated from Equation (2.47), which applies unchanged, but with the composite compressibility $\alpha_{P}$ computed from Equation (2.56). Figure 2.11 shows the relationship between the relative porosity $\phi / \phi_{0}$ and the pressure drop $\Delta P$ in an unconsolidated medium, and describes the cementing effect of solid phases on the medium behavior.

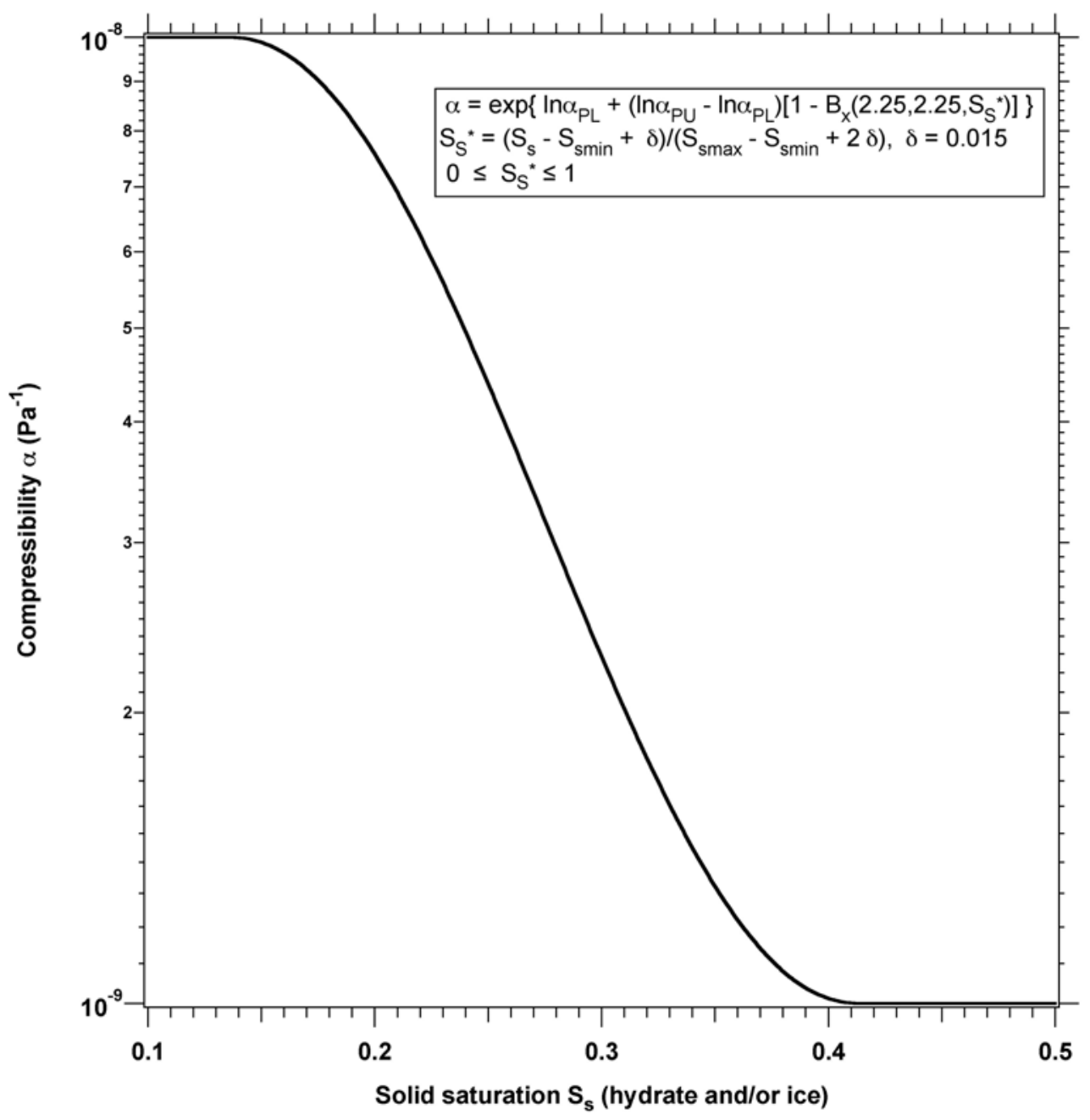

Figure 2.10. Compressibility of an unconsolidated porous medium impregnated with cementing solid phases (ice and/or hydrates). In this example, $S_{S \min }=0.15, S_{S \max }=0.4, \alpha_{P U}=10^{-8} \mathrm{~Pa}^{-1}, \alpha_{P L}$ $=10^{-9} \mathrm{~Pa}^{-1}$ and $\delta=0.015$. 


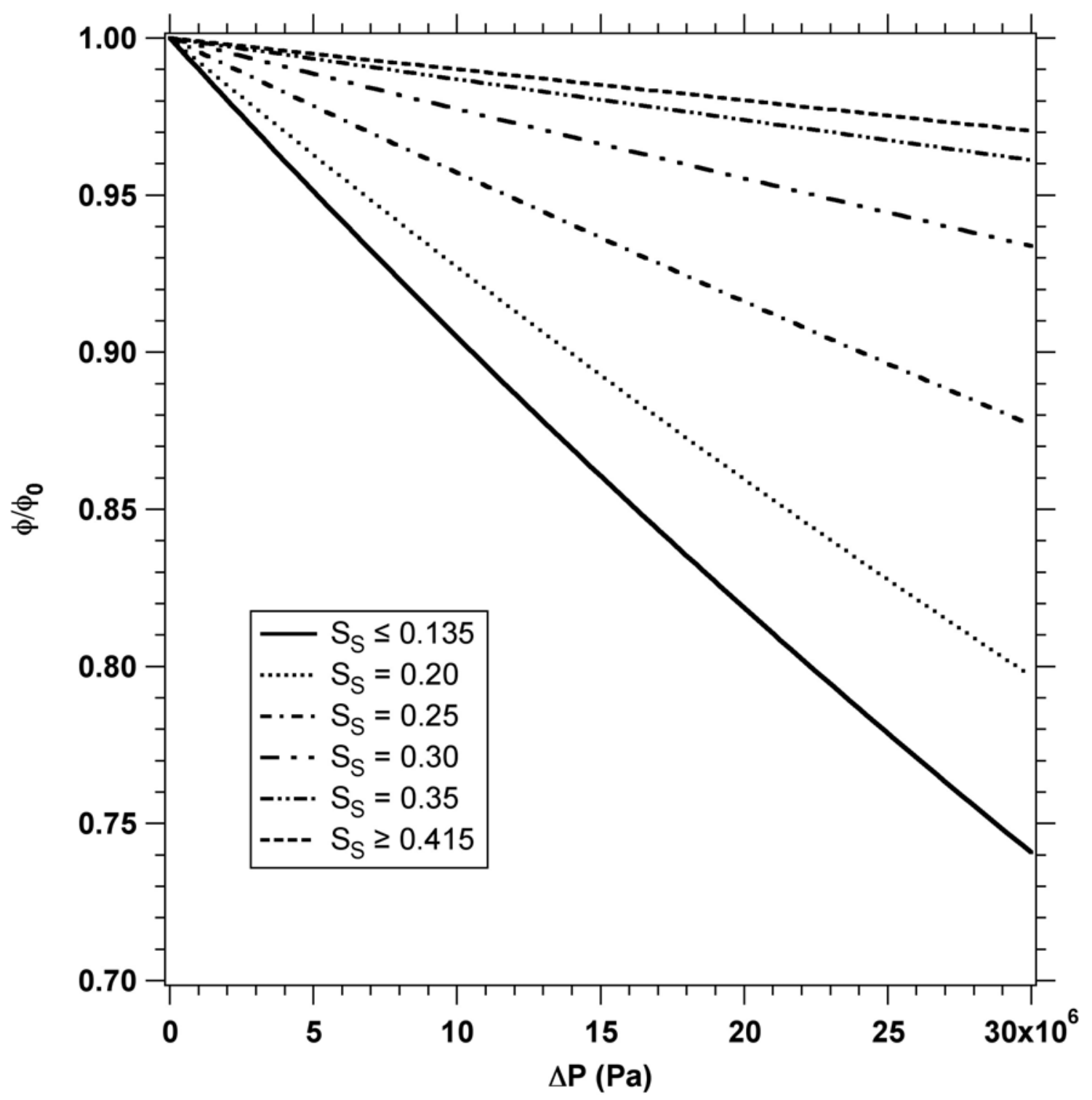

Figure 2.11. Effect of the varying compressibility described in Figure 2.10 on the porosity of an unconsolidated porous medium undergoing depressurization for various levels of saturation $S_{S}$ of cementing solid phases. 


\subsection{Multiphase Diffusion}

\subsubsection{General Considerations}

Molecular diffusion plays a minor role in many subsurface flow processes, but may become a significant and even dominant mechanism for mass transport when advective velocities are small. Diffusive flux is usually written as being proportional to the gradient in the concentration of the diffusing component (Fick's law)

$$
\mathbf{f}=-d \nabla C
$$

where $\mathrm{d}$ is an effective diffusivity, which in general will depend on properties of the diffusing component, the pore fluid, and the porous medium. The concentration variable $\mathrm{C}$ may be chosen in a number of different ways (mass per unit volume, moles per unit volume, mass or mol fraction, etc.) [Bird et al., 1960; de Marsily, 1986].

The basic Fick's law in Equation (2.46) works well for diffusion of tracer solutes that are present at low concentrations in a single phase aqueous solution at rest with respect to the porous medium. However, many subtleties and complications arise when multiple components diffuse in a multiphase flow system. Effective diffusivities in general may depend on all concentration variables, leading to non-linear behavior especially when some components are present in significant (non-tracer) concentrations. Additional nonlinear effects arise from the dependence of tortuosity on phase saturations, and from coupling between advective and diffusive transport. For gases the Fickian model has serious limitations even at low concentrations, which prompted the development of the "dusty gas" model that entails a strong coupling between advective and diffusive transport [Mason and Malinauskas, 1983; Webb, 1998], and accounts for molecular streaming effects (Knudsen diffusion) that become very important when the mean free 
path of gas molecules is comparable to pore sizes. Further complications arise for components that are both soluble and volatile, in which case diffusion in aqueous and gaseous phases may be strongly coupled via phase partitioning effects. An extreme case is the well-known enhancement of vapor diffusion in partially saturated media, which is attributed to pore-level phase change effects [Cass et al., 1984; Webb and Ho, 1998a, b].

\subsubsection{Diffusion Formulation in TOUGH+}

The diffusion formulation and treatment in TOUGH+ follows closely that in TOUGH2 [Pruess et al., 1999]. Because of the difficulties mentioned above, it is not possible to formulate a model for multiphase diffusion that would be accurate under all circumstances. We have used a pragmatic approach in which diffusive flux of component $\kappa$ in phase $\beta$ (= liquid, gas) is written as

$$
\mathbf{f}_{\beta}^{\kappa}=-\phi \tau_{0} \tau_{\beta} \rho_{\beta} d_{\beta}^{\kappa} \nabla X_{\beta}^{\kappa}
$$

where $\phi$ is porosity, $\tau_{0} \tau_{\beta}$ is the tortuosity which includes a porous medium dependent factor $\tau_{0}$ and a coefficient that depends on phase saturation $S_{\beta}, \tau_{\beta}=\tau_{\beta}\left(S_{\beta}\right), \rho_{\beta}$ is density,

$d_{\beta}^{\kappa}$ is the diffusion coefficient of component $\kappa$ in bulk fluid phase $\beta$, and $X_{\beta}^{\kappa}$ is the mass fraction of component $\kappa$ in phase $\beta$. For later developments it will be useful to define a single diffusion strength factor which combines all material constants and tortuosity factors into a single effective multiphase diffusion coefficient, as follows.

$$
\Sigma_{\beta}^{\kappa}=\phi \tau_{0} \tau_{\beta} \rho_{\beta} d_{\beta}^{\kappa}
$$

For general two-phase conditions, the total diffusive flux is then given by

$$
\mathbf{f}^{\kappa}=-\Sigma_{A}^{\kappa} \nabla X_{A}^{\kappa}-\Sigma_{G}^{\kappa} \nabla X_{G}^{\kappa}
$$


The saturation dependence of tortuosity is not well known at present. For soils the Millington and Quirk [1961] model has frequently been used [Jury et al., 1983; Falta et al., 1989]

$$
\tau_{0} \tau_{\beta}=\phi^{1 / 3} S_{\beta}^{10 / 3}
$$

which yields non-zero tortuosity coefficients as long as phase saturation is non-zero. It stands to reason that diffusive flux should vanish when a phase becomes discontinuous at low saturations, suggesting that saturation-dependent tortuosity should be related to relative permeability; e.g. $\tau_{\beta}\left(S_{\beta}\right) \approx k_{r \beta}\left(S_{\beta}\right)$. However, for components that partition between liquid and gas phases more complex behavior may be expected. For example, consider the case of a volatile and water-soluble compound diffusing under conditions of low gas saturation where the gas phase is discontinuous. In this case we have $k_{r G}\left(S_{G}\right)=0$ (because $S_{G}<S_{r g}$ ), and $k_{r A}\left(S_{A}=1-S_{G}\right)<1$, so that a model equating saturationdependent tortuosity to relative permeability would predict weaker diffusion than in single-phase liquid conditions. For compounds with significant volatility this would be unrealistic, as diffusion through isolated gas pockets would tend to enhance overall diffusion relative to single-phase liquid conditions.

\subsection{Description of Flow in Fractured Media}

Figure 2.12 illustrates the classical double-porosity concept for modeling flow in fractured-porous media as developed by Warren and Root [1963]. Matrix blocks of low permeability are embedded in a network of interconnected fractures. Global flow in the reservoir occurs only through the fracture system, which is described as an effective porous continuum. Rock matrix and fractures may exchange fluid (or heat) locally by 
means of 'interporosity flow', which is driven by the difference in pressures (or temperatures) between matrix and fractures. Warren and Root approximated the interporosity flow as being quasi-steady, with rate of matrix-fracture interflow proportional to the difference in (local) average pressures.

The quasi-steady approximation is applicable to isothermal single-phase flow of fluids with small compressibility, where pressure diffusivities are large, so that pressure changes in the fractures penetrate quickly all the way into the matrix blocks. However, for multiphase flows, or coupled fluid and heat flows, the transient periods for interporosity flow can be very long (tens of years). In order to accurately describe such flows it is necessary to resolve the driving pressure, temperature, and mass fraction gradients at the matrix/fracture interface. In the method of "multiple interacting continua" (MINC) [Pruess and Narasimhan, 1982; 1985), resolution of these gradients is achieved by appropriate subgridding of the matrix blocks, as shown in Figure 2.13. The MINC concept is based on the notion that changes in fluid pressures, temperatures, phase compositions, etc., due to the presence of sinks and sources (production and injection wells) will propagate rapidly through the fracture system, while invading the tight matrix blocks only slowly. Therefore, changes in matrix conditions will (locally) be controlled by the distance from the fractures. Fluid and heat flow from the fractures into the matrix blocks, or from the matrix blocks into the fractures, can then be modeled by means of one-dimensional strings of nested grid blocks, as shown in Figure 2.13. 


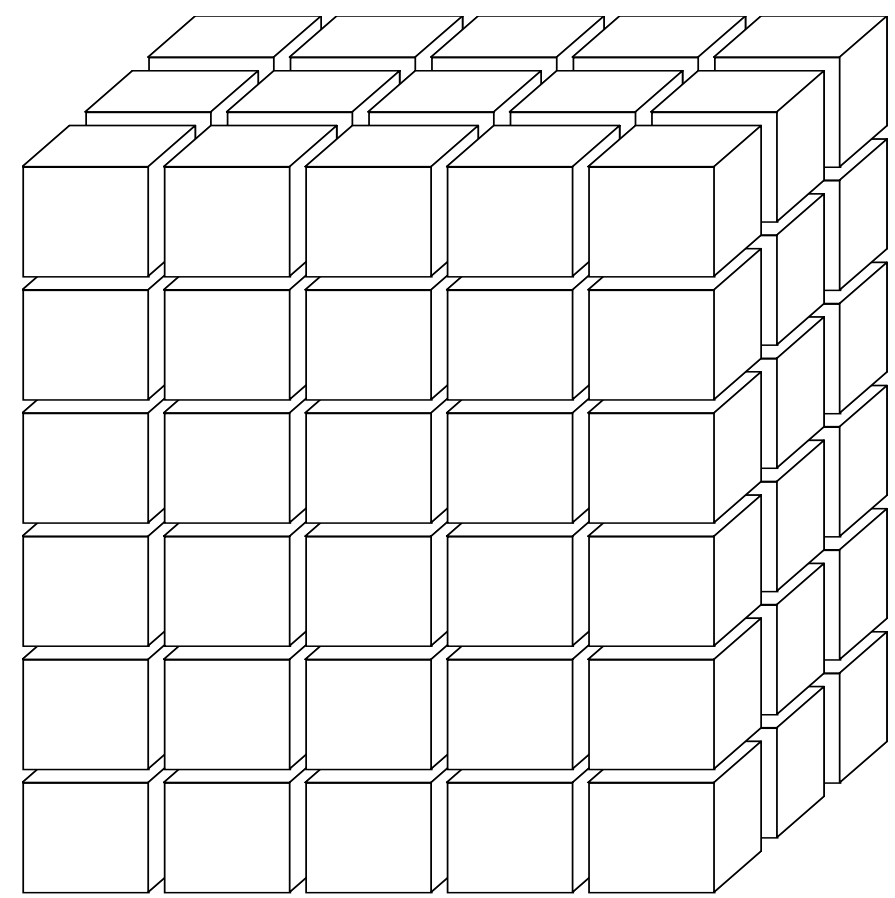

Figure 2.12. Idealized double porosity model of a fractured porous medium.

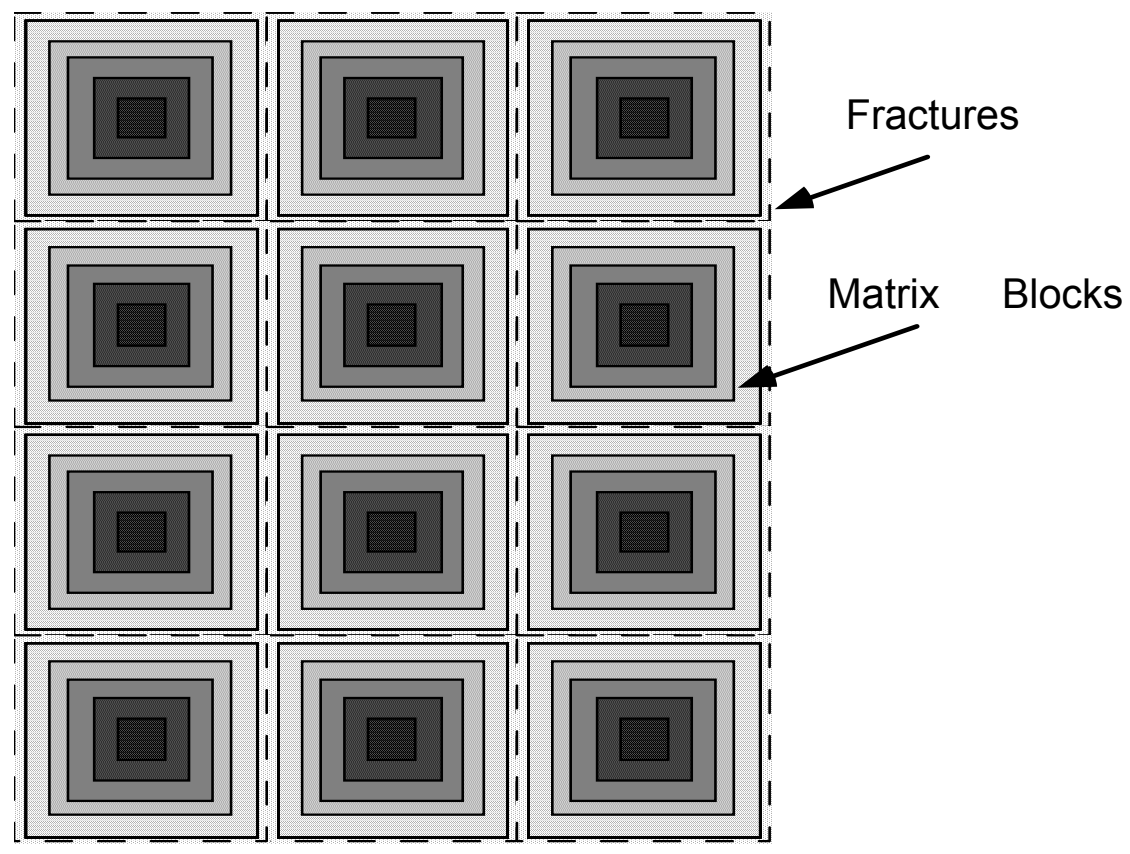

Figure 2.13. Subgridding in the method of "multiple interacting continua" (MINC). 
In general it is not necessary to explicitly consider subgrids in all of the matrix blocks separately. Within a certain reservoir subdomain (corresponding to a finite difference grid block), all fractures will be lumped into continuum \# 1 , all matrix material within a certain distance from the fractures will be lumped into continuum \# 2, matrix material at larger distance becomes continuum \# 3, and so on. Quantitatively, the subgridding is specified by means of a set of volume fractions $\operatorname{VOL}(j), j=1, \ldots, \mathrm{J}$, into which the primary porous medium grid blocks are partitioned. The MINC-process in the MeshMaker.f95 (a companion code distributed with TOUGH+, see Section 7) operates on the element and connection data of a porous medium mesh to calculate, for given data on volume fractions, the volumes, interface areas, and nodal distances for a secondary fractured medium mesh. The information on fracturing (spacing, number of sets, shape of matrix blocks) required for this is provided by a proximity function PROX(x) which expresses, for a given reservoir domain $V_{o}$, the total fraction of matrix material within a distance $\mathrm{x}$ from the fractures. If only two continua are specified (one for fractures, one for matrix), the MINC approach reduces to the conventional doubleporosity method. Full details are given in a separate report [Pruess, 1983].

The MINC-method as implemented in the MeshMaker. $f 95$ code can also describe global matrix-matrix flow. Figure 2.14 shows the most general approach, often referred to as dual permeability, in which global flow occurs in both fracture and matrix continua. It is also possible to permit matrix-matrix flow only in the vertical direction. For any given fractured reservoir flow problem, selection of the most appropriate gridding scheme must be based on a careful consideration of the physical and geometric 
conditions of flow. The MINC approach is not applicable to systems in which fracturing is so sparse that the fractures cannot be approximated as a continuum.

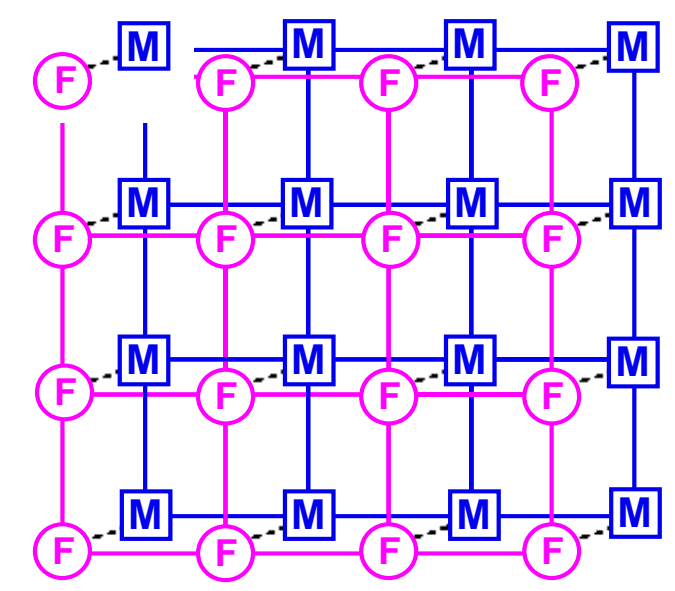

Figure 2.14. Flow connections in the "dual permeability" model. Global flow occurs between both fracture $(F)$ and matrix $(\mathrm{M})$ grid blocks. In addition there is F-M interporosity flow. 


\subsection{Design and Implementation of TOUGH+HYDRATE}

\subsection{Primary Variables}

The thermodynamic state and the distribution of the mass components among the four possible phases are determined from the hydrate equation of state. Following the standard approach employed in the TOUGH2 [Pruess et al., 1999] family of codes, in $\mathbf{T}+\mathbf{H}$ the system is defined uniquely by a set of $N_{\kappa}$ primary variables (where $\kappa$ denotes the number of mass and heat components under consideration, see Section 2.2) that completely specifies the thermodynamic state of the system. Although the number $N_{k}$ of the primary variables is initially set at the maximum expected in the course of the simulation and does not change during the simulation, the thermodynamic quantities used 
as primary variables can change in the process of simulation to allow for the seamless consideration of emerging or disappearing phases and components.

A total of 26 states (phase combinations) covering the entire phase diagram in Figure 2.5 are described in $\mathbf{T}+\mathbf{H}$. Of those, 13 correspond to the equilibrium hydration reaction option, and 13 to the kinetic hydration reaction option. The primary variables used for the various phase states without inhibitor are listed in Tables 3.1 and 3.2, respectively. For systems with an inhibitor, the additional primary variable is $X_{-} i_{-} A$, (corresponding to $X_{A}^{i}$, i.e., the mass fraction of the inhibitor in the aqueous phase). The option set for either equilibrium or kinetic hydration reactions is complete, although some of the phase states are only possible under laboratory conditions and difficult to reach under any conditions of gas production from dissociating natural hydrate deposits.

The primary variables in Tables $\mathbf{3 . 1}$ and 3.2 are necessary and sufficient to uniquely define the $\mathrm{H}_{2} \mathrm{O}-\mathrm{CH}_{4}$ system. Note that the lack of equilibrium in the kinetic model introduces an additional degree of freedom, and consequently necessitates an additional equation. This requires special care to ensure that the resulting system is not overdefined when the hydrate and/or gas phases are not present. 
Table 3.1. Primary Variables in Equilibrium Hydrate Simulations without Inhibitor*.

\begin{tabular}{|c|c|c|c|c|}
\hline Phase & $\begin{array}{c}\text { State } \\
\text { Identifier }\end{array}$ & $\begin{array}{c}\text { Primary } \\
\text { Variable } 1\end{array}$ & $\begin{array}{c}\text { Primary } \\
\text { Variable } 2 \\
\end{array}$ & $\begin{array}{c}\text { Primary } \\
\text { Variable } 3\end{array}$ \\
\hline 1-Phase: G & Gas & $P$ gas & $Y \_m \_G$ & $T$ \\
\hline 1-Phase: A & Aqu & $P$ & $X \_m \_A$ & $T$ \\
\hline 2-Phase: $A+G$ & AqG & $P$ gas & $S \_a q u$ & $T$ \\
\hline 2-Phase $\mathrm{I}+\mathrm{G}$ & IcG & $P$ gas & S_ice & $T$ \\
\hline 2-Phase $\mathrm{H}+\mathrm{G}$ & GsH & $P$ gas & $S \_$gas & $T$ \\
\hline 2-Phase: $\mathrm{A}+\mathrm{H}$ & AqH & $P$ & $S \_a q u$ & $T$ \\
\hline 2-Phase: $A+I$ & AqI & $P$ & $S \_a q u$ & $X \_m \_A$ \\
\hline 2-Phase: $\mathrm{I}+\mathrm{H}$ & IcH & $P$ & S_ice & $T$ \\
\hline 3-Phase: $\mathrm{A}+\mathrm{H}+\mathrm{G}$ & AGH & $S \_g a s$ & $S \_a q u$ & $T$ \\
\hline 3-Phase: $\mathrm{A}+\mathrm{I}+\mathrm{G}$ & AIG & P_gas & $S \_a q u$ & S_gas \\
\hline 3-Phase: $\mathrm{A}+\mathrm{I}+\mathrm{H}$ & AIH & $P$ & $S \_a q u$ & S_ice \\
\hline 3-Phase: $\mathrm{I}+\mathrm{H}+\mathrm{G}$ & IGH & S_gas & S_ice & $T$ \\
\hline $\begin{array}{l}\text { Quadruple Point: } \\
\qquad \mathrm{I}+\mathrm{H}+\mathrm{A}+\mathrm{G}\end{array}$ & QuP & $S \_g a s$ & $S \_a q u$ & S_ice \\
\hline
\end{tabular}

Where the possible primary variables are: $P$, pressure $[\mathrm{Pa}] ; P \_$gas, gas pressure $[\mathrm{Pa}] ; T$, temperature [C]; $X m_{-} A$, mass fraction of $\mathrm{CH}_{4}$ dissolved in the aqueous phase [-]; $Y{ }_{-}{ }_{-} G$, mass fraction of $\mathrm{CH}_{4}$ dissolved in the gas phase [-]; $S \_a q u$, liquid saturation [-]; $S \_$gas, gas saturation [-]; $S_{-}$ice, ice saturation [-]; $X_{-} i \_A$, mass fraction of inhibitor dissolved in the aqueous phase [-].

*For inhibitor: $X \_i_{-} A$ becomes the $3^{\text {rd }}$ primary variable, and the $3^{\text {rd }}$ primary variable (as listed in this table) becomes the $4^{\text {th }}$ primary variable. 
Table 3.2. Primary Variables in Kinetic Hydrate Simulations without Inhibitor*.

\begin{tabular}{|c|c|c|c|c|c|}
\hline Phase & $\begin{array}{c}\text { State } \\
\text { Identifier } \\
\end{array}$ & $\begin{array}{c}\text { Primary } \\
\text { Variable } 1 \\
\end{array}$ & $\begin{array}{c}\text { Primary } \\
\text { Variable } 2 \\
\end{array}$ & $\begin{array}{c}\text { Primary } \\
\text { Variable } 3 \\
\end{array}$ & $\begin{array}{c}\text { Primary } \\
\text { Variable } 4 \\
\end{array}$ \\
\hline 1-Phase: G & Gas & $P$ gas & $Y \_m \_G$ & S_hyd & $T$ \\
\hline 1-Phase: A & Aqu & $P$ & $X \_m \_A$ & S_hyd & $T$ \\
\hline 2-Phase: $A+G$ & AqG & $P$ gas & $S \_a q u$ & S_hyd & $T$ \\
\hline 2-Phase $\mathrm{I}+\mathrm{G}$ & IcG & $P$ gas & S_ice & S_hyd & $T$ \\
\hline 2-Phase $\mathrm{H}+\mathrm{G}$ & GsH & $P$ gas & S_gas & S_ice & $T$ \\
\hline 2-Phase: $\mathrm{A}+\mathrm{H}$ & AqH & $P$ & $S \_a q u$ & $X \_m \_A$ & $T$ \\
\hline 2-Phase: $A+I$ & AqI & $P$ & $S \_a q u$ & $X \_m \_A$ & $T$ \\
\hline 2-Phase: $\mathrm{I}+\mathrm{H}$ & IcH & $P$ & S_ice & S_gas & $T$ \\
\hline 3-Phase: $\mathrm{A}+\mathrm{H}+\mathrm{G}$ & AGH & $P$ gas & $S \_a q u$ & $S \_$gas & $T$ \\
\hline 3-Phase: $\mathrm{A}+\mathrm{I}+\mathrm{G}$ & AIG & $P$ gas & $S_{-} a q u$ & S_hyd & S_gas \\
\hline 3-Phase: $\mathrm{A}+\mathrm{I}+\mathrm{H}$ & AIH & $P$ & $S \_a q u$ & S_ice & $T$ \\
\hline 3-Phase: $\mathrm{I}+\mathrm{H}+\mathrm{G}$ & IGH & $P$ gas & $S \_g a s$ & S_ice & $T$ \\
\hline $\begin{array}{l}\text { Quadruple Point: } \\
\text { I }+\mathrm{H}+\mathrm{A}+\mathrm{G}\end{array}$ & QuP & P_gas & $S \_a q u$ & S_gas & S_ice \\
\hline
\end{tabular}

Where the possible primary variables are: $P$, pressure $[\mathrm{Pa}] ; P \_g a s$, gas pressure $[\mathrm{Pa}] ; T$, temperature [C]; $X m_{-} A$, mass fraction of $\mathrm{CH}_{4}$ dissolved in the aqueous phase [-]; $Y{ }_{-}{ }_{-} G$, mass fraction of $\mathrm{CH}_{4}$ dissolved in the gas phase [-]; $S \_a q u$, liquid saturation [-]; $S \_g a s$, gas saturation [-]; $S \_h y d$, hydrate saturation [-]; $S \_i c e$, ice saturation [-]; $X \_i \_A$, mass fraction of inhibitor dissolved in the aqueous phase [-].

*For inhibitor: $X \_I A$ becomes the 4 th primary variable, and the $4^{\text {th }}$ primary variable (as listed in this table) becomes the $5^{\text {th }}$ primary variable. 


\subsection{Space and Time Discretization}

The continuum equations (2.3) are discretized in space using the integral finite difference method (IFD) [Edwards, 1972; Narasimhan and Witherspoon, 1976]. Introducing appropriate volume averages, we have

$$
\int_{V_{n}} M d V=V_{n} M_{n}
$$

where $M$ is a volume-normalized extensive quantity, and $M_{n}$ is the average value of $M$ over $V_{n}$. Surface integrals are approximated as a discrete sum of averages over surface segments $A_{n m}$ :

$$
\int_{\Gamma_{n}} \mathbf{F}^{\kappa} \bullet \mathbf{n} d \Gamma=\sum_{m} A_{n m} F_{n m}
$$

Here $F_{n m}$ is the average value of the (inward) normal component of $\mathbf{F}$ over the surface segment $A_{n m}$ between volume elements $V_{n}$ and $V_{m}$. The discretization approach used in the integral finite difference method and the definition of the geometric parameters are illustrated in Figure 3.1.

The discretized flux is expressed in terms of averages over parameters for elements $V_{n}$ and $V_{m}$. For the basic Darcy flux term in Equation (2.15), we have

$$
F_{\beta, n m}=-k_{n m}\left[\frac{k_{r \beta} \rho_{\beta}}{\mu_{\beta}}\right]_{n m}\left[\frac{P_{\beta, n}-P_{\beta, m}}{D_{n m}} \rho_{\beta, n m} g_{n m}\right]
$$

where the subscripts $(\mathrm{nm})$ denote a suitable averaging at the interface between grid blocks $\mathrm{n}$ and $\mathrm{m}$ (interpolation, harmonic weighting, upstream weighting). $D_{n m}=D_{n}+D_{m}$ is the distance between the nodal points $n$ and $m$, and $g_{n m}$ is the component of gravitational 
acceleration in the direction from $m$ to $n$. Discretization of diffusive fluxes raises some subtle issues, and is discussed separately in Section 3.5.

Substituting Equations. (3.1) and (3.2) into the governing Equation (2.3), a set of firstorder ordinary differential equations in time is obtained.

$$
\frac{d M_{n}^{\kappa}}{d t}=\frac{1}{V_{n}} \sum_{m} A_{n m} F_{n m}^{\kappa}+q_{n}^{\kappa}
$$

Time is discretized as a first-order finite difference, and the flux and sink and source terms on the right-hand side of Equation (3.4) are evaluated at the new time level, $t^{k+1}=$ $t^{k}+\Delta t$, to obtain the numerical stability needed for an efficient calculation of strongly nonlinear problems (such as the ones involving multiphase flow and phase changes). This treatment of flux terms is known as fully implicit, because the fluxes are expressed in terms of the unknown thermodynamic parameters at time level $t^{k+l}$, so that these unknowns are only implicitly defined in the resulting equations [Peaceman, 1977].
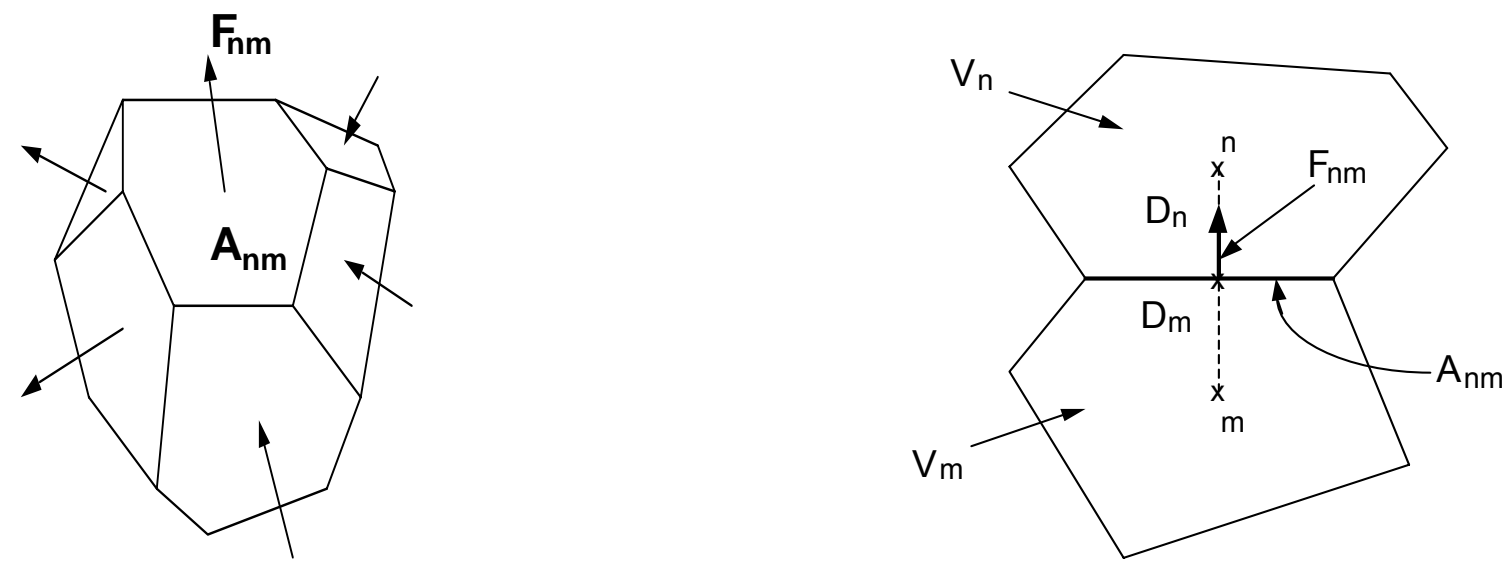

Figure 3.1. Space discretization and geometry data in the integral finite difference method. 
The time discretization results in the following set of coupled non-linear, algebraic equations

$$
R_{n}^{\kappa, k+1}=M_{n}^{\kappa, k+1}-M_{n}^{\kappa, k}-\frac{\Delta t}{V_{n}}\left(\sum_{m} A_{n m} F_{n m}^{\kappa, k+1}+V_{n} q_{n}^{\kappa, k+1}\right)=0
$$

where we have introduced residuals $R_{n}^{\kappa, k+1}$. For each volume element (grid block) $V_{n}$, there are $N_{\kappa}$ equations, so that for a system discretized into $N_{E}$ grid blocks, Equation (3.5) represents a total of $N_{\kappa} \times N_{E}$ coupled non-linear equations.

\subsection{The Newton-Raphson Iteration}

The unknowns of Equation (3.5) are the $N_{\kappa} \times N_{E}$ independent primary variables $\left\{x_{i}\right.$; $\left.\mathrm{i}=1, \ldots, N_{\kappa} \times N_{E}\right\}$ which completely define the state of the flow system at time level $t^{k+l}$. These equations are solved by Newton/Raphson iteration, which is implemented as follows. We introduce an iteration index $p$ and expand the residuals $R_{n}^{\kappa, k+1}$ in Equation (3.5) at iteration step $(p+1)$ in a Taylor series in terms of those at index $p$, i.e.,

$$
R_{n}^{\kappa, k+1}\left(x_{i, p+1}\right)=R_{n}^{\kappa, k+1}\left(x_{i, p}\right)+\left.\sum_{\mathrm{i}} \frac{\partial R_{n}^{\kappa, k+1}}{\partial x_{i}}\right|_{p}\left(x_{i, p+1}-x_{i, p}\right)+\ldots=0
$$

Retaining only terms up to first order, we obtain a set of $N_{\kappa} \times N_{E}$ linear equations for the increments $\left(x_{i, p+1}-x_{i, p}\right)$ :

$$
-\left.\sum_{\mathrm{i}} \frac{\partial R_{n}^{\kappa, k+1}}{\partial x_{i}}\right|_{p}\left(x_{i, p+1}-x_{i, p}\right)+\ldots=R_{n}^{\kappa, k+1}\left(x_{i, p}\right)
$$

All terms $\partial R_{n} / \partial x_{i}$ in the Jacobian matrix are evaluated by numerical differentiation. 
Equation (3.7) is solved by sparse direct matrix methods or iteratively by means of preconditioned conjugate gradients [Moridis and Pruess, 1995; 1998; Pruess et al., 1999]. Iteration is continued until the residuals $R_{n}^{\kappa, k+1}$ are reduced below a preset convergence tolerance according to:

$$
\left|\frac{R_{n, p+1}^{\kappa, k+1}}{M_{n, p+1}^{\kappa, k+1}}\right| \leq \varepsilon_{1}
$$

The default (relative) convergence criterion is $\varepsilon_{1}=10^{-5}$ (TOUGH+ input parameter RE1, see Section 10). When the accumulation terms are smaller than $\varepsilon_{2}(\mathrm{TOUGH}+$ input parameter RE2, default $\varepsilon_{2}=1$, see Section 10 ), an absolute convergence criterion is imposed,

$$
\left|R_{n, p+1}^{\kappa, k+1}\right| \leq \varepsilon_{1} \varepsilon_{2}
$$

The number of iterations to convergence varies with the nonlinearity of the problem. For well-behaved problems, convergence is usually attained in 3--4 iterations. If convergence cannot be achieved within a certain number of iterations (default 8 , see Section 10), the time step size $\Delta t$ is reduced and a new iteration process is started.

\subsection{Implications of the Space Discretization Approach}

It is appropriate to add some comments about the space discretization technique in TOUGH+. The entire geometric information of the space discretization in Equation (3.5) is provided in the form of a list of grid block volumes $V_{n}$, interface areas $A_{n m}$, nodal distances $D_{n m}$ and components $g_{n m}$ of gravitational acceleration along nodal lines. There 
is no reference whatsoever to a global system of coordinates, or to the dimensionality of a particular flow problem.

The discretized equations are in fact valid for arbitrary irregular discretizations in one, two or three dimensions, and for porous as well as for fractured media. This flexibility should be used with caution, however, because the accuracy of solutions depends upon the accuracy with which the various interface parameters in equations such as (3.3) can be expressed in terms of average conditions in grid blocks. A general requirement is that there exists approximate thermodynamic equilibrium in (almost) all grid blocks at (almost) all times [Pruess and Narasimhan, 1985]. For systems of regular grid blocks referenced to global coordinates in cylindrical $(r, z)$ and/or Cartesian $(x, y, z)$ systems, Equation (3.5) is identical to a conventional finite difference formulation [Peaceman, 1977; Moridis and Pruess, 1992].

\subsection{Space Discretization of Diffusive Fluxes}

Space discretization of diffusive flux in multiphase conditions raises some subtle issues. A finite difference formulation for total diffusive flux, Equation (3.10), may be written as

$$
\left(\mathbf{f}^{\kappa}\right)_{n m}=-\left(\sum_{A}^{\kappa}\right)_{n m} \frac{\left(X_{A}^{\kappa}\right)_{m}-\left(X_{A}^{\kappa}\right)_{n}}{D_{n m}}-\left(\sum_{G}^{\kappa}\right)_{n m} \frac{\left(X_{G}^{\kappa}\right)_{m}-\left(X_{G}^{\kappa}\right)_{n}}{D_{n m}}
$$

This expression involves the as yet unknown diffusive strength coefficients $\left(\Sigma_{A}^{\kappa}\right)_{n m}$ and $\left(\Sigma_{G}^{\kappa}\right)_{n m}$ at the interface, which must be expressed in terms of the strength coefficients in the participating grid blocks. Invoking conservation of diffusive flux across the interface between two grid blocks leads in the usual way to the requirement of harmonic weighting 
of the diffusive strength coefficients. However, such weighting may in general not be applied separately to the diffusive fluxes in gas and liquid phases, because these may be strongly coupled by phase partitioning effects. This can be seen by considering the extreme case of diffusion of a water-soluble and volatile compound from a grid block in single-phase gas conditions to an adjacent grid block that is in single-phase liquid conditions. Harmonic weighting applied separately to liquid and gas diffusive fluxes would result in either of them being zero, because for each phase effective diffusivity is zero on one side of the interface. Thus total diffusive flux would vanish in this case, which is unphysical. In reality, tracer would diffuse through the gas phase to the gasliquid interface, would establish a certain mass fraction in the aqueous phase by dissolution, and would then proceed to diffuse away from the interface through the aqueous phase. Similar arguments can be made in the less extreme situation where liquid saturation changes from a large to a small value rather than from 1 to 0 , as may be the case in the capillary fringe, during infiltration events, or at fracture-matrix interfaces in variably saturated media.

TOUGH + features the fully coupled approach employed in TOUGH2 [Pruess et al., 1999], in which the space-discretized version of Equation (3.10) of the total multiphase diffusive flux Equation (2.49) is re-written in terms of an effective multiphase diffusive strength coefficient and a single mass fraction gradient. Choosing the liquid mass fraction for this we have

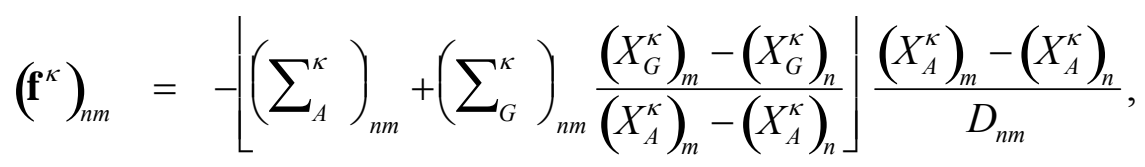


where the gas phase mass fraction gradient has been absorbed into the effective diffusive strength term (in braces). As is well known, flux conservation at the interface then leads to the requirement of harmonic weighting for the full effective strength coefficient. In order to be able to apply this scheme to the general case where not both phases may be present on both sides of the interface, we always define both liquid and gas phase mass fractions in all grid blocks, regardless of whether both phases are present. Mass fractions are assigned in such a way as to be consistent with what would be present in an evolving second phase. This procedure is applicable to all possible phase combinations, including the extreme case where conditions at the interface change from single phase gas to single phase liquid. Note that, if the diffusing tracer exists in just one of the two phases, harmonic weighting of the strength coefficient in Equation (3.11) will reduce to harmonic weighting of either $\Sigma_{A}^{\kappa}$ or $\Sigma_{G}^{\kappa}$.

The simpler scheme of separate harmonic weighting for individual phase diffusive fluxes is retained as an option.

\subsection{Compiling the TOUGH+HYDRATE Code}

TOUGH+HYDRATE is written in standard FORTRAN 95/2003. It has been designed for maximum portability, and runs on any computational form (Unix and Linux workstations, PC, Macintosh) for which such compilers are available. Running $\mathbf{T}+\mathbf{H}$ involves compilation and linking of the following code segments and in the following order:

$$
\text { T_Hydrate_Definitions.f95 (*) }
$$


Segment providing default parameter values describing the basic attributes of the equation of state (i.e., number of components, number of phases, etc.)

(2)

T_Allocate_Memory.f95

Segment responsible for the dynamic memory allocation (following input describing the size of the problem) and dimensioning of most arrays needed by the code, in addition to memory deallocation of unnecessary arrays.

(3)

T_Untility_Functions.f95

Segment that includes all utility functions (including a wide variety of mathematical functions, table interpolation routines, sorting algorithms, etc.).

\section{(4) T_H2O_Properties.f95}

Segment that includes (a) all the water-related constants (parameters), and (b) procedures describing the water behavior and thermophysical properties/processes in its entire thermodynamic phase diagram.

\section{(5) T_Media_Properties.f95}

Segment that describes the hydraulic and thermal behavior of the geologic medium (porous or fractured), i.e., capillary pressure and relative permeability under multiphase conditions, interface permeability and mobility, and interface thermal conductivity.

(6) T_RealGas_EOS.f95

Segment that includes (a) the important constants (parameters) of all hydrate-forming gases, and (b) procedures describing the equation of state (EOS) of real gases (pure or mixtures) using any of the Peng-Robinson, Redlich-Kwong, or Soave-Redlich-Kwong cubic EOS model. The 
procedures in this segment compute the following parameters and processes: compressibility, density, fugacity, enthalpy (ideal and departure), internal energy (ideal and departure), entropy (ideal and departure), thermal conductivity, viscosity, binary diffusion coefficients, solubility in water, and heat of dissolution in water.

Segment that describes the properties and processes of the $\mathrm{CH}_{4}$-hydrate. It includes procedures that describe the $P$ - $T$ relationship along the threephase equilibrium regimes of the $\mathrm{CH}_{4}$-hydrate phase diagram (see Figure 2.4) and compute the heat of the hydration reaction, the hydrate density and enthalpy, and the reaction rate when kinetic formation/dissociation are invoked.

(8)

T_Geomechanics.f95 (*)

Segment that describes the geomechanically-induced changes on the flow properties of the porous media. These include porosity $\phi$ changes caused by pressure and/or temperature variations, intrinsic permeability $k$ changes caused by porosity changes, and scaling of capillary pressures $P_{c a p}$ to reflect changes in $\phi$ and $k$. The $\phi$ and $k$ changes are computed using either simplified of full geomechanical models. When the simplified model is invoked, $\phi$ is a function of (a) $P$ and the pore compressibility $\alpha_{P}$ and (b) of $T$ and the pore thermal expansivity $\alpha_{T}$, while (c) $k$ changes are estimated using emprirical relationships (see Section 8). Changes in $\phi$ and $k$ can also be computed by using a full geomechanical model, which can be optionally coupled with TOUGH+.

(9) T_Hydrate_Specifics.f95 (*)

Segment that includes procedures specific to the hydrate simulation, such as the reading of hydrate-specific inputs, the preparation of hydratespecific output files, and the computation of the thermal conductivity in 
hydrate-bearing media. Generic procedures and operator extension -which override (overload) the standard procedures used by TOUGH+ for the simulation of non-hydrate problems - are defined in this segment, which does not include any procedures describing the hydrate equation of state.

\section{(10) T_Main.f95}

Main program that organizes the calling sequence of the high-level events in the simulation process, and includes the writing of important general comments in the standard output files, timing procedures, and handling of files needed by the code and/or created during the code execution.

\section{(11) T_Hydrate_EOS.f95 (*)}

Segment that describes the equation of state of the $\mathrm{CH}_{4}$-hydrate, assigns initial conditions, computes the thermophysical properties of the hydratebearing medium, and determines phase changes and the state of the system from the 22 possible options (see Section 3.1). This segment also includes the procedure that computes the elements of the Jacobian matrix for the Netwon-Raphson iteration (see Sections 3.3).

\section{(12) T Matrix Solvers.f 95}

A linear algebra package that includes all the direct and iterative solvers availabse in $\mathrm{TOUGH}+($ see Section 10$)$.

\section{(13) T_Executive.f95}

The executive segment of $\mathrm{TOUGH}+$. It includes the procedures that advance the time in the simulation process, estimate the time-step size for optimum performance, populate the matrix arrays and invoke the solvers of the Jacobian, invoke special linear algebra for matrix pre-processing in cases of very demanding linear algebra problems, compute mass and energy balances, compute rates in sources and sinks, compute binary 
diffusion coefficients, write special output files, and conduct other miscellaneous operations.

\section{(14) T_Inputs.f95}

This segment includes the procedures involved in the reading of the general input files needed for $\mathrm{TOUGH}+$ simulations. It does not include any procedure reading hydrate-related data (this is accomplished in the TFx_Hydrate_Spcfc.f95 segment).

The segments denoted by $(*)$ are specific to the hydrate problem, and are needed by $\mathbf{T}+\mathbf{H}$. All other segments are common to all TOUGH+ simulations.

Additionally, $\mathbf{T}+\mathbf{H}$ is distributed with the Meshmaker. $\mathbf{f 9 5}$ FORTRAN code, which used to be part of the main code in the TOUGH and TOUGH2 simulators, but is a separate entity in the TOUGH+ family of codes. Meshmaker.f95 is used for the space discretization (gridding) of the domain of the problem under study (see Section 7).

NOTE: In compiling $\mathbf{T}+\mathbf{H} \vee 1.0$, it is important that the free-format source code option be invoked for proper compilation. 


\subsection{Input Data Requirements}

In this section, we discuss in detail the input requirements for TOUGH+HYDRATE simulations. To ensure backward compatibility with older TOUGH2 input files (a functional requirement for $\mathrm{TOUGH}+$ ), the input data for $\mathbf{T}+\mathbf{H}$ are a superset of the input data used in conventional TOUGH2 simulations. Thus, the inputs for the current version of TOUGH+ are similar in type, input format, and parameter representation with those in TOUGH2 (an advanced, keyword-based data input system, will be made available in future $\mathrm{TOUGH}+$ releases).

\subsection{Input Procedure}

The input procedure in the current version of $\mathrm{TOUGH}+$ remains similar in many aspects to that of TOUGH2 [Pruess et al., 1991]. Input data can be provided in a flexible manner by means of one or several ASCII data files. The TOUGH+ inputs are in 
standard metric (SI) units, such as meters, seconds, kilograms, ${ }^{\circ} \mathrm{C}$, and the corresponding derived units, such as Newtons, Joules, and Pascal $=\mathrm{N} / \mathrm{m}^{2}$ for pressure.

In the TOUGH + input file data are organized in data blocks that are defined by keywords. Quite often, only the first five characters of the keywords typed in columns 15 (see Table 4.1) are read, because these are sufficient to recognize the data block. While the contents of the various blocks are described in detail in Sections 5-12, here we describe some important records/keywords, and provide some general comments about their occurrence and arrangement in the input file.

\subsubsection{Data Block/Keyword TITLE}

The first record of the input file in any TOUGH+ simulation is TITLE, which includes a header of up to 132 characters. This record is necessary for any simulation to begin.

\subsubsection{Keyword/Record START or RANDOMN}

The use of the START or RANDOMN record/keyword typed in columns 1-5 allows a more flexible initialization (the boldface in RANDOMN indicates the portion of the keyword used by the code). More specifically, when START or RANDOMN is present, data initialization in the INCON data block (or file) can be in arbitrary order, and need not be present for all grid blocks (in which case defaults will be used). Without START or RANDOMN, there must be a one-to-one correspondence between the data in data blocks ELEME and INCON. 


\subsubsection{Keyword/Record ENDCY}

A record with the ENDCY keyword typed in columns 1-5 closes the TOUGH+ input file and instructs the code to initiate the simulation.

\subsubsection{Keyword/Record ENDFI}

The presence of the ENDFI keyword in columns 1-5 is an alternative (to ENDCY) ending keyword in a TOUGH + standard input file. Presence of ENDFI keyword causes the simulation to be skipped after printing basic input information. This is a useful option when the simulation is limited to an attempt to obtain some basic information of the properties and conditions of the system in its initial state.

\subsubsection{General Comments on the Structure of TOUGH+ Standard Input Files}

Every TOUGH + input file must (a) begin with the record/keyword TITLE (providing a problem title of up to 80 characters) and (b) end either with the record ENDCY, or, alternatively, with the record ENDFI (if no flow simulation is to be carried out). Data records beyond ENDCY (or ENDFI) will be ignored.

Some data blocks, such as those specifying reservoir domains (ROCKS or MEDIA), volume elements (ELEME), connections (CONNE), and sinks/sources (GENER), have a variable number of records, while others have a fixed number of records. The end of variable-length data blocks is indicated by a blank record. The data blocks between the TITLE and ENDCY/ENDFI keywords can be provided in arbitrary order, except for the data block ELEME, which must precede (if either is present) the blocks CONNE and EXT- 
INCON. The blocks ELEME and CONNE must either be both provided through the standard input file, or must both be absent, in which case alternative means for specifying geometry data will be employed (see Section 7). The data block GENER can be omitted if there are no sinks and sources in the problem. If the keyword START or RANDOMN is present (see Section 4.1.2), the data block INCON can be incomplete, with elements (grid blocks) in arbitrary order, or can be absent altogether.

Elements for which no initial conditions are specified in INCON will then be assigned domain-specific initial conditions from (a) block INDOM (if present), or (b) from the data block EXT - INCON (if present), or (c) from the 'generic' initial conditions given in block PARAM, along with default porosities given in block ROCKS. If START or RANDOMN is not present, INCON must contain information for all elements, in exactly the same order as they are listed in block ELEME.

Between data blocks, the standard TOUGH + input file may include an arbitrary number of records that do not begin with any of the TOUGH+ keywords. This is useful for inserting comments about problem specifications directly into the input file. TOUGH+ will gather all these comments and will print the first 50 such records in the output file.

Much of the data handling in TOUGH+ is accomplished by means of disk files, which can be edited and modified using any text editor. The initialization of the arrays for geometry, generation, and initial condition data is always made from the disk files MESH (or MINC), GENER, and INCON. A user can either provide these files at execution time, or they can be written from TOUGH+ input data during the initialization phase of the program. 
If the data blocks GENER and/or INCON are not provided in the standard input file, and if no disk files GENER and/or INCON are present, defaults will take effect (no generation; initial conditions from block INDOM, or from block EXT-INDOM, or defaults from block PARAM). To ensure that these defaults are used, the disk files GENER and/or INCON (from a previous run) must be removed from the execution environment/directory. A safe way to use default generation and initial conditions is to specify dummy data blocks in the input file, consisting of one record with GENER or INCON, followed by a blank record.

The format of data blocks ELEME, CONNE, GENER, and INCON is basically the same (see Section 7) when these data are provided as disk files and when they are given as part of the input file. However, specification of these data through the input file rather than as disk files offers some added conveniences, which are useful when a new simulation problem is initiated. For example, a sequence of identical items (volume elements, connections, sinks or sources) can be specified through a single data record. Also, indices used internally for cross-referencing elements, connections, and sources will be generated internally by TOUGH+ rather than having them provided by the user. INCON, GENER, and INCON disk files written by TOUGH+ can be merged into an input file without changes, keeping the cross-referencing information. 
Table 4.1. TOUGH+ input data blocks.

\begin{tabular}{|c|c|c|}
\hline Keyword & Sec. & Function \\
\hline TITLE $\left(1^{\text {st }}\right.$ record $)$ & 4.1.1 & Data record (single line) with simulation title \\
\hline MEMORY & 5.1 & Dynamic memory allocation \\
\hline HYDRATE & 6.1 & Parameters describing hydrate properties and behavior \\
\hline ROCKS or MEDIA & 6.2 & Hydrogeologic parameters for various reservoir domains \\
\hline $\begin{array}{l}\text { RPCAP or } \\
\text { WETTABILITY }\end{array}$ & 6.3 & $\begin{array}{l}\text { Optional; parameters for relative permeability and capillary } \\
\text { pressure functions }\end{array}$ \\
\hline DIFFU & 6.4 & Optional; diffusivities of mass components \\
\hline *ELEME & 7.1 & List of grid blocks (volume elements) \\
\hline *CONNE & 7.2 & List of flow connections between grid blocks \\
\hline MESHMAKER & 7.3 & Optional; parameters for internal grid generation \\
\hline INDOM & 8.1 & Optional; list of initial conditions for specific reservoir domains \\
\hline *INCON & 8.2 & Optional; list of initial conditions for specific grid blocks \\
\hline EXT - INCON & 8.3 & Optional; list of initial conditions for specific grid blocks \\
\hline BOUNDARIES & 8.6 & Optional; provides time-variable conditions at specific boundaries \\
\hline *GENER & 9.1 & Optional; list of mass or heat sinks and sources \\
\hline PARAM & 10.1 & $\begin{array}{l}\text { Computational parameters; time stepping and convergence } \\
\text { parameters; program options }\end{array}$ \\
\hline SOLVR & 10.2 & Optional; specifies parameters used by linear equation solvers. \\
\hline TIMES & 11.2 & Optional; specification of times for generating printout \\
\hline ELEM_TIME_SERIES & 11.3 & Optional; specifies grid blocks for desired time series data \\
\hline CONX_TIME_SERIES & 11.4 & Optional; specifies connections for desired time series data \\
\hline SS_TIME_SERIES & 11.5 & Optional; specifies sinks/sources for desired time series data \\
\hline SUBDOMAINS & 11.6 & Optional; specifies grid subdomains for desired time series data \\
\hline NOVER & 11.6 & Optional; suppresses printout of version numbers and dates \\
\hline START or RANDOMN & 4.1.2 & Optional; one data record for more flexible initialization \\
\hline ENDCY(last record) & 4.1 .3 & Record closes TOUGH+ input file and initiates simulation \\
\hline ENDFI(last record) & 4.1.4 & $\begin{array}{l}\text { Alternative for closing TOUGH+ input file which causes flow } \\
\text { simulation to be skipped. }\end{array}$ \\
\hline
\end{tabular}

Blocks labeled with * can be provided as separate disk files and omitted from input file. 


\subsection{Memory Specification and Allocation}

\subsection{Data Block MEMORY}

This is the data block that reads the data for the dynamic memory allocation in the

$\mathbf{T}+\mathbf{H}$ simulation. This block must always follow the TITLE record.

$\underline{\text { Record MEMORY . } 1}$

This record must always include the following header:

MEMORY

$\underline{\text { Record MEMORY . } 2}$

Reads the character variable EOS Name according to FORMAT (A15). For hydrate simulations, the value of EOS_Name can be either HYDRATEEQUILIBRIUM or HYDRATE-KINETIC. Note that this parameter does not necessarily provide an indication of the type of hydrate simulation (equilibrium vs. kinetic) to follow, but is used strictly to allocate memory to the pertinent storage arrays. 


\section{Record MEMORY . 3}

The following information is provided in MEMORY . 3 using a free format:

$$
\text { NumCom, NumEq, Numphases, binary_diffusion }
$$

These parameters are defined as follows:

NumCom

NumEq

NumPhases

binary_diffusion
Integer denoting the number of mass components (see Section 2)

Integer denoting the number of equations

Integer denoting the number of phases.

Note that for in the case of hydrates, the code resets internally the input value of NPH to 4 . This ensures that the simulation can fully describe the evolution of the hydrate-bearing system should ice emerge.

Logical variable indicating whether binary diffusion is to be considered (binary_diffusion = .TRUE.) or ignored (binary_diffusion = . FALSE.).

The following combinations are possible for hydrate simulations in $\mathbf{T}+\mathbf{H}$ :

(1) (NumCom, NumEq, NumPhases) $=(2,3,4)$ :

Equilibrium hydrate reaction, no inhibitor

(2) (NumCom, NumEq, NumPhases) $=(3,4,4)$ :

Equilibrium hydrate reaction with inhibitor

(3)

$$
\begin{aligned}
& \text { (NumCom, NumEq, NumPhases })=(3,4,4): \\
& \text { Kinetic hydrate reaction, no inhibitor } \\
& (\text { NumCom, NumEq, NumPhases })=(4,5,4): \\
& \text { Kinetic hydrate reaction with inhibitor }
\end{aligned}
$$

\section{Record MEMORY . 4}

In MEMORY . 4, the following parameters are read using a free format:

coordinate_system Character variable describing the coordinate system used in the study. It can assume the values

' Cartesian' or 'Cylindrical'. 

Max_NumElem
Max_NumConx
ElemNameLength
active_conx_only
Integer variable defining the maximum number of elements (cells, gridblocks) in the discretized simulation domain
Integer variable defining the maximum number of connections in the discretized simulation domain.
Integer variable defining the number of characters in the element names. It may be either 5 or 8 (default is 5 in v1.0).
Logical variable indicating whether the simulation will be halted after determining the active connections in the grid. This feature is useful when running a simulation that uses a subset of the elements of a large grid without correspondingly adjusting the connections, and is designed to reduce the very large memory requirements for the connection-related dynamic arrays. When active_conx_only $=$. TRUE., the simulation stops once the active connections (involving only the elements defined in the element list) are determined. The active connections are stored in a new file called Active_Connection_File. Then the simulation can be run using the new connection list, thus having much lower memory requirements. For a thorough discussion of elements and connections in TOUGH+, see Section 7.

Information on the concepts of elements and connections can be found in Pruess et al. [1999]. As a general rule, MaxNum_Conx > ND*MaxNum_Elem, where ND is the dimensionality of the problem.

\section{Record MEMORY . 5}

The integer variable Max_NumSS (declaring the maximum number of expected sources and sinks) is read using a free format.

\section{Record MEMORY . 6}

In this record, the integer variable Max_NumMedia is read using a free format. This variable represents the maximum number of geologic media with different properties to be considered in the simulations.

\section{Record MEMORY . 7}


In MEMORY . 7, the following logical variables are read using a free format:

$$
\begin{aligned}
& \text { element_by_element_properties Logical variable (flag) } \\
& \text { indicating whether each gridblock has its own } \\
& \text { hydraulic properties ( } \phi \text { and } k \text { ), in which case they } \\
& \text { are read on an element-by-element basis (see } \\
& \text { Sections } 7 \text { and 8). This feature is necessary (a) in } \\
& \text { the simulation of very heterogeneous systems, } \\
& \text { and/or (b) when } k \text { changes in response to pressure } \\
& \text { and/or temperature variations, or in response to } \\
& \text { changes in the geomechanical regime of the system. }
\end{aligned}
$$

porosity_perm_dependence Logical variable (flag) indicating whether the intrinsic permeability $k$ in a given element is to change as a function of changing porosity $\phi$. As discussed earlier, $\phi$ can change in response to $P$ and/or $T$ changes that are determined from simple or complex geomechanical models (see Section 2).

When porosity_perm_dependence $=$. FALSE., then $k$ is unaffected by changes in $\phi$. Whenporosity_perm_dependence $=$. TRUE ., then $k$ is readjusted internally to reflect the effect of changes in $\phi$ that are estimated using either an empirical model (see Equation 2.51, Section 2) or a full geomechanical model.

Note that when porosity_perm_dependence $=$. TRUE. , the variable element_by_element_properties is set internally to . TRUE . because activation of the porosity_perm_dependence feature results in element-specific hydraulic properties. 
scaled_capillary_pressureLogical variable (flag) indicating whether the capillary pressure $P_{\text {cap }}$ will be scaled to reflect variations in $\phi$ and $k$.

Activation of this feature by setting scaled_capillary_pressure $=$.TRUE . may be needed (a) in highly heterogeneous systems in which the element-specific properties vary significantly from those described by the average (expected) values of the porous medium (as specified in Section 6), and/or (b) when the significant variations in $\phi$ and $k$ are experienced in the course of the simulation (e.g., when porosity_perm_dependence=.TRUE.).

\section{$\underline{\text { Record MEMORY . } 8}$}

In MEMORY. 8, the following logical variables are read using a free format: coupled_geochemistry_ Logical variable (flag) indicating whether the simulation involves coupled flow, thermal and geochemical processes. This feature is activated by setting coupled_geochemistry to . TRUE. when geochemical processes are considered in a $\mathrm{TOUGH}+$ simulation. Because it is not possible to invoke such geochemical processes, coupled_geochemistry is set internally to . FALSE . in $\mathbf{T}+\mathbf{H}$ simulations.

property_update Character variable (flag) indicating the type of property update when coupled geochemical processes are involved in $\mathrm{TOUGH}+$ simulations. Because of the reasons discussed above, it is not used in $\mathbf{T}+\mathbf{H}$ simulations.

\section{Record MEMORY . 9}

In MEMORY. 9, the following logical variables are read using a free format: coupled_geomechanics Logical variable (flag) indicating whether the simulation involves coupled flow, thermal and geomechanical processes. Activation of this feature by setting coupled_geomechanics $=$. TRUE. indicates the use of a complex geomechanical 
model to describe the relationship between hydraulic media properties ( $\phi$ and $k$ ) and geomechanical parameters (such as stresses and strains). This complex geomechanical model overrides the simplified models (based on pore compressibility and expansivity) that are standard in TOUGH+ (see Section 2). In its current implementation, the geomechanical code invoked by the TOUGH+ code is the FLAC3D commercial simulator (ITASCA, 2006). When present in the TOUGH+ directory, FLAC3D . exe (the executable of the FLAC3D code) is treated as a C subroutine that is called from the $\mathrm{TOUGH}+$ simulator (written in FORTRAN95/2003) when the variable coupled geomechanics $=$. TRUE. . When coupled_geomechanics =. FALSE. the simplified geomechanical models are invoked, even when FLAC3D . exe is present in the $\mathrm{TOUGH}+$ directory.

When coupled_geomechanics =. TRUE., and the the FLAC3D. exe executable is present in the TOUGH+ directory, the following files are created: (a) To_GMech, containing the data (pressure, temperatures and phase saturations) that are provided to the FLAC3D geomechanical code from the $\mathrm{TOUGH}+$ simulator for use in the computation of the geomechanical properties of the system, and (b) the Fr_Gmech file, containing the data (mainly stresses and strains) supplied by the FLAC3D code for use by the $\mathrm{T}+\mathrm{H}$ simulator for the computation of the variable (geomechanicallydependent) hydraulic properties $\phi$ and $k$. These two files are necessary for communication between the two codes, because the lack of shared memory (disallowed because of intellectual property concerns) makes data exchange by means of these two external files as the only viable option.

property_update

Character variable (flag) denoting the manner of property update as a result of interdependent changes in the hydraulic (flow) and geomechanical properties. The variable property_update can assume the following values: ' Continuous ', indicating continuous property update (i.e., in every Newtonian iteration of every timestep) and 
participation in the Jacobian matrix;

' Iteration ', indicating property updates in every Newtonian iteration of every timestep, but without any contribution to the Jacobian; and ' Timestep ', indicating a single property update at the end of each timestep and no contribution to the Jacobian. The option property_update $=$ ' Cont inuous ' yields the most accurate solutions that are accurate over any pressure range and media geomechanical property range but results in longer execution times, while the option property update $=$ 'Timestep ' leads to faster solutions, but which are acceptably accurate in less compressible media and for mild pressure drops.

If a property up value other than the three decribed above is read, then an error message is printed and the simulation is aborted.

Integer variable defining the number of geomechanical parameters that are to be provided by the FLAC3D code (to be read by the TOUGH+ simulator from the Fr_Gmech file), and which will be used to estimate the updated $\phi$ and $k$. While FLAC3D can provide a very large number of geomechanical properties and parameters of interest, in the current version of the $\mathrm{T}+\mathrm{H}$ only two are (stress and strain) are needed. Thus, NumGeomechParam must be set to 2 in this record when the FLAC3D geomechanical model is invoked for hydrate simulations that involve coupled geomechanical effects. 


\subsection{Physical Properties of System}

In this section, the physical properties of the system, including hydrate properties (block HYDRATE), rock properties (block ROCKS or MEDIA), relative permeability and capillary pressure properties (block RPCAP), properties specifying multi-component diffusion (block DIFFU) are described, followed by the description of a procedure for introducing block-by-block permeability modification.

\subsection{Data Block HYDRATE}

The parameters describing the hydrate properties and behavior are provided here. Note that free format is used to read the data in this data block. 


\section{Record HYDRATE . 1}

The number of component hydrates NCom is read in this card. Because of the dearth of quantitative information on the behavior of complex hydrates (as explained in Section 1.2), $\mathrm{NCOm}=1$ in this version of $\mathbf{T}+\mathbf{H}$.

\section{Record HYDRATE . 2}

This card reads the following data:

nameG The name of the hydrate-forming gas

hydrN The corresponding hydration number - see Equation (2.1)

moleF The mole fraction in the composite hydrate (for pure hydrates, moleF $=1$ )

\section{Record HYDRATE . 3}

This record includes $\mathrm{N}$ ThC, which is the number of coefficients of the polynomial describing the dependence of the thermal conductivity $K_{H}$ of the hydrate on temperature $T$.

\section{$\underline{\text { Record HYDRATE . } 4}$}

This card includes the coefficients $A_{n}\left(n=0, \ldots, N_{k}, N_{k}=\mathrm{N} \_\mathrm{ThC}-1\right)$ of the thermal conductivity polynomial

$$
K_{H}=A_{0}+A_{1} T+A_{2} T^{2}+\ldots+A_{n} T^{n}
$$

\section{Record HYDRATE . 5}

This record includes $\mathrm{N}_{\mathrm{SPH}} \mathrm{Sp}$, which is the number of coefficients of the polynomial describing the dependence of the specific heat $C_{H}$ of the hydrate on temperature $T$.

\section{Record HYDRATE . 6}

This card provides the coefficients $B_{n}\left(n=0, \ldots, N_{c}, N_{c}=\mathrm{N}_{-} \mathrm{SpH}-1\right)$ of the specific heat polynomial

$$
C_{H}=B_{0}+B_{1} T+B_{2} T^{2}+\ldots+B_{n} T^{n}
$$

\section{Record HYDRATE . 7}

This record includes $\mathrm{N}$ Rho, which is the number of coefficients of the polynomial describing the dependence of the hydrate density $\rho_{H}$ on temperature $T$. Record HYDRATE . 8 
This card provides the coefficients $D_{n}\left(n=0, \ldots, N_{c}, N_{c}=\mathrm{N}_{-}\right.$Rho -1$)$ of the hydrate density polynomial.

$$
\rho_{H}=D_{0}+D_{1} T+D_{2} T^{2}+\ldots+D_{n} T^{n}
$$

\section{Record HYDRATE . 9}

This card reads the following inhibitor-related data:

\begin{tabular}{|c|c|}
\hline inhibitor_flag & $\begin{array}{l}\text { Logical variable, (flag) indicating presence of an } \\
\text { inhibitor when inhibitor_flag }=\text {. TRUE.. } \\
\text { Note that no additional variable values are read past } \\
\text { this point if inhibitor_flag }=\text {. FALSE.. }\end{array}$ \\
\hline Max_TShift & $\begin{array}{l}\text { Real variable describing the inhibitor-induced } \\
\text { reference temperature depression }- \text { see Equation } \\
(2.36) \text {. When salt }(\mathrm{NaCl}) \text { is the inhibitor in } \\
\text { question, Max_Tshift }=2.0 \mathrm{E} 0 .\end{array}$ \\
\hline Y_atMax_TShift & $\begin{array}{l}\text { Real variable describing the reference inhibitor } \\
\text { mole fraction in the aqueous phase, corresponding } \\
\text { to Max_TShift }- \text { see Equation }(2.36) \text {. For NaCl, } \\
\text { Y_atMax_Tshift }=1.335 \mathrm{E}-2 \text {. }\end{array}$ \\
\hline InhibitorMW & $\begin{array}{l}\text { Real variable describing the molecular weight of the } \\
\text { inhibitor }\left[\mathrm{g} \mathrm{mol}^{-1}\right] \text {. For } \mathrm{NaCl} \text {, InhibitorMW = } \\
5.8448 \mathrm{E} 1 \mathrm{~g} \mathrm{~mol}^{-1} \text {. }\end{array}$ \\
\hline InhibitorDens & $\begin{array}{l}\text { Real variable describinig the inhibitor density }[\mathrm{kg} \\
\left.\mathrm{m}^{-3}\right] \text {. For } \mathrm{NaCl} \text {, InhibitorDens }=2.6 \mathrm{E} 3 \mathrm{~kg} \\
\mathrm{~m}^{-3} \text {. }\end{array}$ \\
\hline InhibitorEnthSol & $\begin{array}{l}\text { Real variable describinig the specific enthalpy of } \\
\text { the inhibitor dissolution in water }\left[\mathrm{J} \mathrm{kg}^{-1}\right] \text {. For NaCl, } \\
\text { InhibitorEnthSol }=6.6479 \mathrm{E} 4 \mathrm{~J} \mathrm{~kg}^{-1} \text {. }\end{array}$ \\
\hline InhibitorCpCoeff & $\begin{array}{l}\text { Real array of dimension } 3 \text {, containing the } \\
\text { coefficients of the inhibitor specific heat } C_{i} \text { vs. } T \\
\text { quadratic equation } C_{i}=C_{0}+C_{1} T+C_{2} T^{2} \text { (see } \\
\text { Equation 2.14). }\end{array}$ \\
\hline
\end{tabular}




\section{Record HYDRATE . 10}

The integer variable EquationOption in Card HYDRATE. 10 is an option for the selection of the equation describing the $P e$ vs. $T e$ and $\Delta H^{0}$ vs. Te relationships (see discussion in Section 2.9). For Equationoption $=0$, the modified equations of Moridis [2003] are used. For Equationoption = 1, the equations of Kamath [1984] are employed. For EquationOption = 2, the $P e$ vs. Te relationship is computed using the equation of Moridis [2003], while the $\Delta H^{0}$ vs. Te relationship is obtained from the Kamath [1984] equation. A value of Equationoption $=2$ is the preferred option.

\section{Record HYDRATE . 11}

The character variable Reaction_Type (LEN = 5) in Card HYDRATE.11 describes the type of hydrate reaction, and can take one of two values. For simulations under equilibrium conditions, Reaction_Type= 'EQUILIBRIUM'. Kinetic hydrate reactions are considered when Reaction_Type $=$ 'KINETIC'.

\section{Record HYDRATE . 12}

The card HYDRATE.12 is read only if Reaction_Type = 'KINETIC', and includes the following kinetic dissociation parameters:

ActivationEnergy Real variable describing the activation energy $\Delta E_{a}$ for the hydrate dissociation $\left[\mathrm{J} \mathrm{mol}^{-1}\right]-$ see Equation (2.6)

IntrinsicRateconstant Real variable describing the intrinsic hydration reaction constant $K_{0}\left[\mathrm{~kg} \mathrm{~m}^{-2} \mathrm{~Pa}^{-1} \mathrm{~s}^{-1}\right]$

Area_Factor Real variable describing the area adjustment factor [dimensionless] - see Equation (2.6)

\subsection{Data Block ROCKS or MEDIA}

This block introduces material parameters for any number of different geologic media (porous or fractured). 
Record ROCKS. 1

Format (A5, I5, 8E10.4)

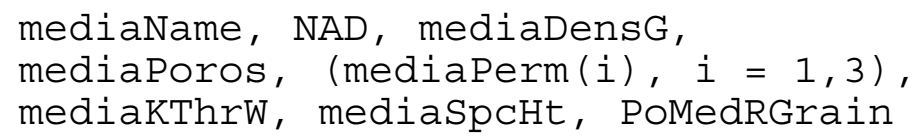

medianame

NAD

mediaDensG

mediaporos
Material name (rock type).

Integer variable; if zero or negative, defaults will take effect for a number of parameters (see below);

$\geq 1$ : will read another data record to override defaults.

$\geq 2$ and $<5$ : will read two more records with domain-specific parameters for relative permeability and capillary pressure functions.

$=5:$ In addition to the three records read for NAD $>$ 2 , an additional record will be read with the coefficients of the porosity polynomial $\phi / \phi=F_{0}+$ $F_{1} \Delta P+F_{2} \Delta P^{2}+\ldots+F_{n} \Delta P^{n}$, where $\phi$ is the reference (initial) default porosity and $\Delta P=P-P_{0}$ is the deviation from the initial pressure $P_{0}$. This equation will be used instead of Equation (2.47) to estimate the effect of pressure on the medium porosity.

$=6:$ In addition to the three records read for NAD $>$ 2 , an additional record will be read with the coefficients of Equations (2.56) and (2.57) that describe the compressibility of an unconsolidated porous medium in the presence of cementing solid phases (such as ice and/or hydrates).

Rock grain density $\left[\mathrm{kg} / \mathrm{m}^{3}\right]$

Default porosity $\phi$ (void fraction) for all elements belonging to domain MediumName for which no other porosity has been specified in block INCON. Option "START" is necessary for using the default porosity. 


\begin{abstract}
mediaPerm( $i), i=1, \ldots, 3 \quad$ Absolute permeabilities along the three principal axes, as specified by ConxKi in block CONNE.
\end{abstract}

mediaKThrw

mediaspcHt

PoMedRGrain
Formation heat conductivity under fully liquidsaturated conditions $\left[\mathrm{W} / \mathrm{m} /{ }^{\circ} \mathrm{C}\right]$.

Rock grain specific heat $\left[\mathrm{J} / \mathrm{kg} /{ }^{\circ} \mathrm{C}\right]$.

Rock grain radius [m]. This is needed for the estimation of the hydrate surface reaction area when kinetic hydrate reactions are invoked. If PoMedRGrain $=0.0 \mathrm{e} \odot$ (e.g., when no value is provided), the $\mathrm{TOUGH}+$ code provides a grain radius estimate using the Kozeny-Carman approximation (see Section 2.4).

Record ROCKS.1.1 (optional, when NAD $\geq 1$ only)

Format (10E10.4)

mediaCompr, mediaExpan, mediaKThrD, mediaTortu, mediaklink, mediaOrgCF, mediaCritsat, mediaPermExpon, mediaBeta, mediaGama

mediaCompr

mediaExpan

mediaKThrD

mediaTortu

mediaKlink
Pore compressibility $\alpha_{P}=(1 / \phi)(\partial \phi / \partial P)_{T}\left[\mathrm{~Pa}^{-1}\right]$, see Equation (2.47) - default $\alpha_{P}=0.0$.

Pore expansivity $\alpha_{T}=(1 / \phi)(\partial \phi / \partial T)_{P}\left[1 /{ }^{\circ} \mathrm{C}\right]$, see Equation (2.47) - default $\alpha_{T}=0.0$.

Formation heat conductivity under desaturated conditions $\left[\mathrm{W} \mathrm{m}^{-1} \mathrm{~K}^{-1}\right]-$ default is mediaKThrD $=$ mediakThrW.

Tortuosity factor for binary diffusion. If mediaTortu $=0$, a porosity and saturationdependent tortuosity will be calculated internally from the Millington and Quirk [1961] model, Equation (2.53).

Klinkenberg parameter $b\left[\mathrm{~Pa}^{-1}\right]$ for enhancing gas phase permeability according to the relationship $k_{G}=k_{L}(1+b / P)$, where $k_{G}$ and $k_{L}$ are the effective 
permeabilities of the gas and of the liquid (i.e., the aqueous phase in the case of gas hydrates) phase, respectively (default is 0 ).

mediaOrgCF

mediaCritsat

mediaPermExpon

mediaBeta

mediaGama
Not used for hydrate simulations.

Critical mobile phase saturation $\phi_{c}$, i.e., mobile phase saturation $\left(=S_{A}+S_{G}\right)$ at which the permeability of hydrate and/or ice-bearing medium becomes equal to zero - needed only when the EPM model is invoked (see Sections 2.11 and 2.12). Permeability reduction exponent for solid phasebearing systems - See Sections 2.11 and 2.12, Equation (2.40).

The parameter $\beta$ used for the computation of porosity as a function of geomechanical stresses $\sigma$ according to the equation:

$\phi / \phi_{0}=\phi_{c}+\left(1-\phi_{c}\right) \exp [\beta(\sigma-P)]$

NOTE: This $\phi$ computational option can be invoked only when the option coupled_geomechanics $=$. TRUE . is activated, i.e., when the TOUGH+ simulations are coupled with a full geomechanical model such as the FLAC3D code. If coupled_geomechanics $=$. FALSE ., this parameter is ignored and the variations in $\phi$ are computed from one of the other computational options available in $\mathrm{TOUGH}+$.

The parameter $\gamma$ used for the computation of intrinsic permeability $k$ as an empirical function of variations in the porosity $\phi$ - See Sections 2.12.2.1, Equation (2.51).

Record ROCKS. 1.2 (optional, NAD $\geq 2$ only)

Format (I5, 5X, 7E10.4)

RelPermEquationNum, (RelPermParam(i) , $i=1, \ldots, 7$ )

RelPermEquationNum Integer parameter indicating the type of the relative permeability function of the medium under 
consideration (see detailed discussion in Section 6.3.1).

RelPermParam ( $i), i=1, \ldots, 7$ Real parameters corresponding to the relative permeability function described by the RelPermEquationNum option (see Section 6.3.1).

$\underline{\text { Record ROCKS. } 1.3}$ (optional, NAD $\geq 2$ only)

Format (I5, 5X, 7E10.4)

PcapEquationNum, (PcapParam (i) , $i=1, \ldots 7$ )

PcapEquationNum Integer parameter indicating the type of the capillary pressure function of the medium under consideration (see detailed discussion in Section $6.3 .2)$.

PcapParam(i), $i=1, \ldots 7$ Real parameters corresponding to the capillary pressure function described by the PcapEquationNum option (see Section 6.3.2).

Record ROCKS.1.4 (optional, NAD = 5 only, to be used when the media porosity is described as a polynomial function of the pressure change $\Delta P$ )

Format (I5, 5X, 7E20.13)

$$
\text { PhipolyOrder, (PhiCoeff (i), } i=0, \ldots, 6)
$$

PhiPolyOrder $\quad$ Order $n$ of the polynomial $\phi / \phi=F_{0}+F_{1} \Delta P+$ $F_{2} \Delta P^{2}+\ldots+F_{n} \Delta P^{n}$. For a constant $\phi$, PhiPolyOrder $=0$.

NOTE: $\triangle P$ is in $\mathrm{MPa}$, not the usual $\mathrm{Pa}$ units of pressure.

PhiCoeff (i) ,i=0, .., 6 Coefficients $F_{n}(n=0, \ldots$, PhiPolyOrder $)$ of the $\phi=\phi(\Delta P)$ polynomial.

NOTE: The units of $F_{n}$ must reflect the fact that $\Delta P$ is in $\mathrm{MPa}$. 
Record ROCKS. 1.4 (optional, NAD $=6$ only, to be used when cementing solid phases such as ice and/or hydrates are present in the pores of unconsolidated media - see Section 2.11.3)

Format (10E10.4)

LoComp, SatAtLoComp,

HiComp, SatAtHiComp, Deltasat

LoComp

The lower limit of the medium compressibility $\alpha_{P L}$ $\left[\mathrm{Pa}^{-1}\right]$, corresponding to the full stiffening/strengthening effect of the presence of cementing solid phases such as ice and/or hydrates - see Equation (2.56).

SatAtLoComp $=S_{S \max }$, i.e., the lowest $S_{S}$ saturation at which $\alpha_{P}=$ $\alpha_{P L}$ - see Equations (2.56) and (2.57).

HiComp

The upper limit of the medium compressibility $\alpha_{P U}$ $\left[\mathrm{Pa}^{-1}\right]$, corresponding to the absence of cementing solid phases- see Equation (2.56).

SatAtHiComp $=S_{\text {Smin }}$, i.e., the largest $S_{S}$ saturation at which $\alpha_{P}=$ $\alpha_{P U}-$ see Equations (2.56) and (2.57).

Deltasat

The smoothing factor $\delta$ - see Equation (2.57). A value of $\delta=0.015$ is suggested - see Figure 2.10.

Repeat records ROCKS.1, ROCKS.1.1, ROCKS.1.2, ROCKS.1.3 and ROCKS.1.4 for all the porous/fractured media in the domain under investigation.

Record ROCKS. 2

A blank record closes the ROCKS data block.

Note: The number of media described in ROCKS/MEDIA cannot exceed the number Max_NumMedia specified in the MEMORY data block (See Section 5.1). If this happens, an error message is printed and the simulation is aborted. 


\subsection{Data Block RPCAP}

This block introduces information on relative permeability and capillary pressure functions, which will be applied for all flow domains for which no data were specified in records ROCKS.1.2 and ROCKS.1.3. A catalog of relative permeability and capillary pressure functions is presented in Sections 6.3.1 and 6.3.2, respectively.

Record RPCAP. 1

Format (I5, 5X, 7E10.4)

DefaultRelPermType, (RPD $(i), i=1,7)$

DefaultRelPermType Integer parameter describing the type of the default relative permeability function (see Section 6.3.1).

$(\operatorname{RPD}(i), I=1, \ldots, 7) \quad$ Real parameters corresponding to the relative permeability function selected by the DefaultRelPermType parameter (see Section 6.3.1).

Record RPCAP. 2

Format (I5, 5X, 7E10.4)

DefaultCapPresType, (CPD (I), $i=1,7$ )

DefaultCapPresType Integer parameter describing the type of the default capillary pressure function (see Section 6.3.2).

( $\mathrm{CPD}(i), i=1, \ldots, 7) \quad$ Real parameters corresponding to the capillary pressure function selected by the DefaultCapPresType parameter (see Section 6.3.2).

\subsubsection{Relative Permeability Functions}

6.3.1.1. RelPermEquationNum $=1$ : Linear functions

$k_{r A}$ increases linearly from 0 to 1 in the range $\mathrm{RP}(1) \leq S_{A} \leq \mathrm{RP}(3)$;

$k_{r G}$ increases linearly from 0 to 1 in the range $\mathrm{RP}(2) \leq S_{G} \leq \mathrm{RP}(4)$

Restrictions: $\operatorname{RP}(3)>\operatorname{RP}(1) ; \quad \operatorname{RP}(4)>\operatorname{RP}(2)$. 
6.3.1.2. RelPermequationNum $=2$ : Power functions

$k_{r A}=\left(S_{A}\right)^{n}, \quad k_{r G}=1, \quad$ where $n=\operatorname{RP}(1)$

6.3.1.3. RelPermEquationNum = 3 : Corey's curves [Corey,1954]

$k_{r A}=\hat{S}^{4}, \quad k_{r G}=(1-\hat{S})^{2}\left(1-\hat{S}^{2}\right)$

where $\quad \hat{S}=\frac{\left(S_{A}-S_{i r A}\right)}{\left(1-S_{i r A}-S_{i r G}\right)}$

with $S_{i r A}=\operatorname{RP}(1) ; \quad S_{i r G}=\operatorname{RP}(2)$

Restrictions: $\operatorname{RP}(1)+\operatorname{RP}(2)<1$

6.3.1.4. RelPermEquationNum = 4 : Grant's curves [Grant, 1977]

$k_{r A}=\hat{S}^{4}, \quad k_{r G}=1-k_{r A}$

where $\quad \hat{S}=\frac{\left(S_{A}-S_{i r A}\right)}{\left(1-S_{i r A}-S_{i r G}\right)}$

with $S_{i r A}=\operatorname{RP}(1) ; \quad S_{i r G}=\operatorname{RP}(2)$

Restrictions: $\operatorname{RP}(1)+\operatorname{RP}(2)<1$

6.3.1.5. RelPermEquationNum = 5 : All phases perfectly mobile $k_{r G}=k_{r A}=1$ for all saturations; no parameters

6.3.1.6. RelPermEquationNum $=6:$ Functions of Fatt and Klikoff (1959)

$$
k_{r A}=\left(S^{*}\right)^{3}, \quad k_{r G}=\left(1-S^{*}\right)^{3}
$$

where: $\quad S^{*}=\frac{\left(S_{A}-S_{i r A}\right)}{\left(1-S_{i r A}\right)}$

with $S_{i r A}=\operatorname{RP}(1)$.

Restriction: $\operatorname{RP}(1)<1$. 


\subsubsection{RelPermEquationNum = 7, - 7: van Genuchten-Mualem model [Mualem, 1976; van Genuchten, 1980]}

$$
k_{r A}= \begin{cases}\sqrt{S^{*}}\left\{1-\left(1-\left[S^{*}\right]^{1 / \lambda}\right)^{\lambda}\right\}^{2} & \text { if } S_{A}<S_{m \times A} \\ 1 & \text { if } S_{A} \geq S_{m \times A}\end{cases}
$$

Gas relative permeability can be chosen from among several options. For RelPermEquationNum = 7, it is computed from one of the following three forms, of which the second is the Corey [1954] equation and the third is the modified Stone equation [Stone, 1970] - see Section 6.1.3.9:

$$
k_{r G}=\left\{\begin{array}{rr}
1-k_{r G} & \text { if } S_{i r G}=0 \\
(1-\hat{S})^{2}\left(1-\hat{S}^{2}\right) & \text { if } S_{i r G}>0 \text { and } n_{G} \leq 0 \\
(\tilde{S})^{n_{G}} & \text { if } S_{i r G}>0 \text { and } n_{G}>0
\end{array}\right.
$$

For RelPermEquationNum $=-7$, the gas relative permeability is computed from the model of Parker et al. [1987]:

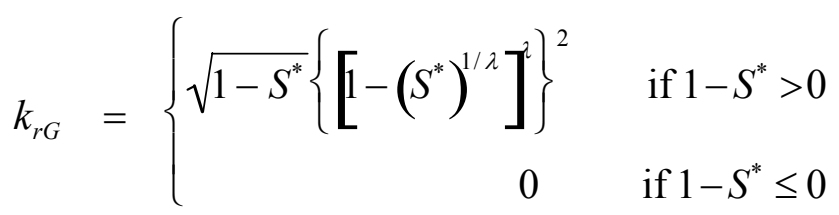

The $k_{r A}$ and $k_{r G}$ estimates are subject to the following restrictions:

$$
0 \leq k_{r A}, k_{r G} \leq 1
$$

Here, $S^{*}=\frac{S_{A}-S_{i r A}}{S_{m \times A}-S_{i r A}}, \quad \hat{S}=\frac{S_{A}-S_{i r A}}{1-S_{i r A}-S_{i r G}} \quad \tilde{S}=\frac{S_{G}-S_{i r G}}{1-S_{i r A}}$

$$
\begin{array}{ll}
\text { Parameters: } & \operatorname{RP}(1)=\lambda \\
& \operatorname{RP}(2)=S_{i r A} \\
\operatorname{RP}(3)=S_{m x A} \\
\operatorname{RP}(4)=S_{i r G} \\
\operatorname{RP}(5)=n_{G}
\end{array}
$$

Notation: Parameter $\lambda$ is $m$ in van Genuchten's notation, with $m=1-1 / n$. 
6.3.1.8. Rel PermequationNum = 8: Function of Verma et al. [1985]

$$
\begin{aligned}
& k_{r A}=\hat{S}^{3}, \quad k_{r G}=A+B \hat{S}+C \hat{S}^{2} \\
& \text { where } \quad \hat{S}=\frac{\left(S_{A}-S_{i r A}\right)}{\left(S_{m x A}-S_{i r A}\right)}
\end{aligned}
$$

Parameters as measured by Verma et al. [1985] for steam-water flow in an unconsolidated sand:

$$
\begin{array}{lll}
S_{i r A}= & \operatorname{RP}(1)=0.2 \\
S_{m x A}= & \operatorname{RP}(2)=0.895 \\
A & = & \operatorname{RP}(3)=1.259 \\
B & = & \operatorname{RP}(4)=-1.7615 \\
C & = & \operatorname{RP}(5)=0.5089
\end{array}
$$

6.3.1.9. RelPermEquationNum = 9 : Modified version of Stone's first threephase relative permeability method [Stone, 1970].

$$
\begin{aligned}
& k_{r A}=\max \left\{0, \min \left\{\left[\frac{S_{A}-S_{i r A}}{1-S_{i r A}}\right]^{n}, 1\right\}\right\}, \\
& k_{r G}=\max \left\{0, \min \left\{\left[\frac{S_{G}-S_{i r G}}{1-S_{i r A}}\right]^{n_{G}}, 1\right\}\right\}, \\
& k_{r H}=0
\end{aligned}
$$

Parameters are $S_{i r A}=\mathrm{RP}(1), S_{i r G}=\mathrm{RP}(2), n=\mathrm{RP}(3), n_{G}=\mathrm{RP}(4)$. Note: When $\operatorname{RP}(4)=0.0 e 0$, it is reset internally to $\operatorname{RP}(4)=\operatorname{RP}(3)$.

The user may modify the source code of TOUGH+ (segment T_Media_Properties.f95) to employ other relative permeability relationships. However, for this task the user needs to be familiar with the concepts of generic processes (and overloading) in object oriented programming languages. 


\subsubsection{Capillary Pressure Functions}

6.3.2.1. PcapEquationNum $=1$ : Linear function

$$
P_{\text {cap }}= \begin{cases}-\mathrm{CP}(1) & \text { for } S_{A} \leq \mathrm{CP}(2) \\ 0 & \text { for } S_{A} \leq \mathrm{CP}(2) \\ -\mathrm{CP}(1) \frac{\mathrm{CP}(3)-S_{A}}{\mathrm{CP}(3)-\mathrm{CP}(2)} & \text { for } \mathrm{CP}(2)<S_{A}<\mathrm{CP}(3)\end{cases}
$$

Restriction: $\mathrm{CP}(3)>\mathrm{CP}(2)$

6.3.2.2. PcapEquationNum $=2$ : Function of Pickens et al. [1979]

$$
P_{\text {cap }}=-P_{0}\left\{\ln \left[\frac{A}{B}\left(1+\sqrt{1-B^{2} / A^{2}}\right)\right]\right\}^{1 / x}
$$

with

$$
A=\left(1+\frac{S_{A}}{S_{A 0}}\right) \frac{\left(S_{A 0}-S_{i r A}\right)}{\left(S_{A 0}+S_{i r A}\right)}, \quad B=1-\frac{S_{A}}{S_{A 0}}
$$

where $P_{0}=\mathrm{CP}(1), S_{i r A}=\mathrm{CP}(2), S_{A 0}=\mathrm{CP}(3), x=\mathrm{CP}(4)$

Restrictions: $0<\mathrm{CP}(2)<1 \bullet \mathrm{CP}(3) ; \mathrm{CP}(4) \neq 0$

6.3.2.3. PcapEquationNum $=3:$ TRUST capillary pressure $[$ Narasimhan et al., 1978]

$$
P_{\text {cap }}= \begin{cases}-P_{e}-P_{0}\left[\frac{1-S_{A}}{S_{A}-S_{i r A}}\right]^{1 / \eta} & \text { for } S_{A}<1 \\ 0 & \text { for } S_{A}<1\end{cases}
$$

where $P_{0}=\mathrm{CP}(1), S_{i r A}=\mathrm{CP}(2), \eta=\mathrm{CP}(3), P_{e}=\mathrm{CP}(4)$

Restrictions: $\mathrm{CP}(2) \geq 0 ; \mathrm{CP}(3) \neq 0$ 
6.3.2.4. PcapequationNum $=4$ : Milly's function $[$ Milly, 1982]

$$
P_{\text {cap }}=-97.783 \times 10^{A}, \quad A=2.26\left(\frac{0.371}{S_{A}-S_{i r A}}-1\right)^{1 / 4}
$$

where $S_{i r A}=\mathrm{CP}(1)$

Restriction: $\mathrm{CP}(1) \geq 0$

6.3.2.5. PcapEquationNum $=6:$ Leverett's function [Leverett, 1941; Udell and Fitch, 1985]

$$
P_{\text {cap }}=-P_{0} \cdot \sigma(T) \cdot f\left(S_{A}\right)
$$

where

$\sigma(T): \quad$ surface tension of water (supplied internally in TOUGH+)

$$
\begin{aligned}
& f\left(S_{A}\right)=1.417\left(1-S^{*}\right)-2.120\left(1-S^{*}\right)^{2}+1.263\left(1-S^{*}\right)^{3} \\
& S^{*}=\frac{\left(S_{A}-S_{i r A}\right)}{\left(1-S_{i r A}\right)}
\end{aligned}
$$

Parameters: $P_{0}=\mathrm{CP}(1), S_{i r A}=\mathrm{CP}(2)$

Restriction: $0 \leq \mathrm{CP}(2)<1$

6.3.2.6. PcapEquationNum = 7 : van Genuchten function [van Genuchten, 1980]

$$
P_{c a p}=-P_{0}\left[\left(S^{*}\right)^{-1 / \lambda}-1\right]^{-\lambda}, \quad S^{*}=\frac{\left(S_{A}-S_{i r A}\right)}{\left(S_{m \times A}-S_{i r A}\right)}
$$

subject to the restriction: $\quad-P_{\max } \leq P_{c a p} \leq 0$

$$
\begin{aligned}
& \text { Parameters: } \quad \mathrm{CP}(1)=\lambda=1-1 / n \\
& \mathrm{CP}(2)=S_{\text {irA }} \text { (should be chosen smaller than the } \\
& \text { corresponding parameter in the relative } \\
& \text { permeability function; see note below.) } \\
& \mathrm{CP}(3)=1 / P_{0}=\alpha / \rho_{w} g
\end{aligned}
$$




$$
\begin{aligned}
& \mathrm{CP}(4)=P_{\text {max }} \\
& \mathrm{CP}(5)=S_{m x A}
\end{aligned}
$$

Notation: Parameter $\lambda$ is $m$ in van Genuchten's notation, with $m=1-1 / n$.

Note on parameter choices: In the van Genuchten's derivation [1980] of the capillary pressure equation, the parameter $S_{i r A}$ for irreducible water saturation is the same in the relative permeability and capillary pressure functions. As a consequence, for $S_{A} \rightarrow S_{i r A}$ we have $k_{r A} \rightarrow 0$ and $P_{\text {cap }} \rightarrow-\infty$, which is unphysical because it implies that the radii of capillary menisci go to zero as liquid phase is becoming immobile (discontinuous). In reality, no special capillary pressure effects are expected when liquid phase becomes discontinuous. Accordingly, we recommend to always define a smaller $S_{i r A}$ for the capillary pressure as compared to that for the relative permeability function.

6.3.2.7. PcapequationNum = 8: Brooks-Corey equation modified to account for effect of hydrate on capillary pressure

$$
P_{\text {cap }}=-F \cdot G \cdot P_{G E}\left(S^{*}\right)^{v}, S^{*}=\frac{\left(S_{A}-S_{i r A}\right)}{\left(1-S_{i r A}\right)}, F=1+A \cdot B x\left(a, b, S_{H}\right)
$$

where

$v \quad=$ exponent with the following restrictions: $\mathrm{v}<0$ and $|\mathrm{v}| \leq 1$;

$P_{G E} \quad=$ gas entry pressure;

$G=$ error function equation that smoothes curve near $\mathrm{S}=0$;

$F \quad=$ factor that describes effect of hydrate on capillary pressure;

$A \quad=$ parameter $>0$;

$B_{x} \quad=$ incomplete beta function;

$a, b \quad=$ input arguments for $B_{x}$;

$S_{H} \quad=$ hydrate saturation.

Parameters: $\quad \mathrm{CP}(1)=P_{G E}, \mathrm{CP}(2)=v, \mathrm{CP}(3)=S_{i r A}$,

$$
\mathrm{CP}(4)=P_{\text {cap, max }}, \mathrm{CP}(5)=A, \mathrm{CP}(6)=a, \mathrm{CP}(7)=b
$$

6.3.2.8. PcapEquationNum $=9:$ No capillary pressure

$P_{c a p} \equiv 0$ for all saturations; no parameters. 


\subsection{Data Block DIFFUSION}

This block reads multicomponent diffusion coefficients.

$\underline{\text { Record DIFFU. } 1}$

Format $(8 \mathrm{E} 10.4)$

(diffusivity $(i, 1), i=1$, NumMobPhases)

diffusivity $(i, 1) \quad$ Diffusion coefficients of mass component \#1 in all mobile phases $i(=1, \ldots$, NumMobPhases $)$.

Record DIFFU. 2

Format(8E10.4)

(diffusivity $(i, 2), i=1$, NumMobPhases)

diffusivity $(i, 2) \quad$ Diffusion coefficients of mass component \#1 in all mobile phases $i(=1, \ldots$, NumMobPhases $)$.

Record DIFFU. 3, DIFFU. 4, etc.

Provide a total of NumCom records with diffusion coefficients for all NumCom mass components. See Section 6.4.1 for additional parameter specifications for diffusion.

If the DIFFU block is invoked, then full multiphase diffusion is considered, which can be a computationally intensive process.

\subsubsection{User Options for Multiphase Diffusion}

The treatment of full multiphase diffusion requires parameter specifications in two different data blocks. First, the user must set binary_diffusion to a value of . TRUE . in data block MEMORY. Diffusivities are input through the data block DIFFU (Section 6.4). See note in Section 6.4 regarding an alternative approach, which allows user to only specify the diffusion coefficient of salt and thus save in computation costs.

Diffusion coefficients for gases depend on pressure and temperature as [Vargaftik, 1975; Walker et al., 1981] 


$$
d_{\beta}^{\kappa}(P, T)=d_{\beta}^{\kappa}\left(P_{0}, T_{0}\right) \frac{P_{0}}{P}\left[\frac{T+273.15}{273.15}\right]^{\theta}
$$

At standard conditions of $P_{0}=1 \mathrm{~atm}=1.01325 \mathrm{bar}, T_{0}=0{ }^{\circ} \mathrm{C}$, the diffusion coefficient for vapor-air mixtures has a value of $2.13 \times 10^{-5} \mathrm{~m}^{2} / \mathrm{s}$; parameter $\theta$ for the temperature dependence is 1.80. TOUGH+ can model a temperature dependence of gas phase diffusion coefficients according to Equation (6.4) by specifying parameter $\theta=$ TEXP in the first record of data block PARAM (see Section 10.1). Presently there are no provisions for inputting different values for the parameter $\theta$ of temperature dependence for different gas phase components.

Tortuosity effects have a porous medium-dependent part $\tau_{0}$ and a saturationdependent part $\tau_{\beta}$, see Equation (2.47). The following three alternative formulations are available.

1. Relative permeability model: for domains for which a tortuosity parameter $\tau_{0}=$ mediaTortu $\neq 0$ is specified in data block ROCKS, tortuosity will be taken as $\tau_{0} \tau_{\beta}\left(S_{\beta}\right)=\tau_{0} k_{r \beta}\left(S_{\beta}\right)$

2. Millington-Quirk model: for domains in which mediaTortu $=0.0 e 0$, the Millington-Quirk model as given in Equation (2.50) will be used.

3. Constant diffusivity: when diffusivities $d_{\beta}^{\kappa}$ are specified as negative numbers, $\tau_{0} \tau_{\beta}=\tau_{0} S_{\beta}$ will be used. Diffusivities will be taken to be the absolute values of the user inputs.

Alternative 3 corresponds to the formulation for gas diffusion that was used in TOUGH2 [Pruess et al., 1999] and in older versions of the code [Pruess, 1987; 1991]. In the 
absence of phase partitioning and adsorptive effects, it amounts to effective diffusivity being approximately equal to $\tau_{0} d_{\beta}^{\kappa}$, independent of saturation. This can be confirmed by noting that the accumulation term in the phase $\beta$ contribution to the mass balance equation for component $\kappa$ is given by $\phi S_{\beta} \rho_{\beta} X_{\beta}^{\kappa}$, approximately canceling out the $\phi S_{\beta} \rho_{\beta}$ coefficient in the diffusive flux.

A crude (saturation-independent) enhancement of vapor diffusion can be chosen by specifying a suitable value, typically of order 1 , for the parameter group $B=\phi \tau_{0} \tau_{\beta}$. This is to be entered as parameter Diffusionstrength in the first record in data block PARAM (see Section 10.1).

As discussed above, two alternative discretization schemes are implemented in TOUGH + . The default option is selected for MOP $(24)=0$ (see Section 10.1) and applies harmonic weighting to the full multiphase effective diffusion strength (see Eq.(2.50)). This includes contributions from gas and aqueous phases, accounts for coupling of diffusion with phase partitioning effects, and can cope with the most general case of diffusion across a phase boundary. As an alternative, by setting MOP $(24)=1$, harmonic weighting can be performed separately for the diffusive fluxes in gas and aqueous phases.

\subsection{Block-by-Block Permeability Modification}

TOUGH + provides a feature that applies permeability modification (PM) coefficients for individual grid blocks according to 


$$
k_{n} \rightarrow k_{n}^{\prime}=k_{n} \cdot \zeta_{n}
$$

Here, $k_{n}$ is the absolute (intrinsic or reference) permeability of grid block (cell, element) $n$, as specified in data block ROCKS or MEDIA for the domain to which that grid block belongs, while $\zeta_{\mathrm{n}}$ is the permeability modification coefficient. The strength of capillary pressure will be automatically scaled according to Leverett [1941] as:

$$
P_{c a p, n} \rightarrow P_{c a p, n}^{\prime}=\frac{P_{c a p, n}}{\sqrt{\zeta_{n}}}
$$

The subroutine Initialize_Perm_Modifiers is called after all MESH data have been processed. The Initialize_Perm_Modifiers routine initializes permeability modifiers and generates informative printout. To engage block-by-block permeability modification, users need to specify a (dummy) domain named "SEED" in block ROCKS or MEDIA. No grid blocks should be assigned to this domain; the presence of domain "SEED" simply serves as a flag to put permeability modification into effect, while data provided in domain "SEED" serve to select different options. Random (spatially uncorrelated) PM data can be internally generated in TOUGH + . Alternatively, externally defined permeability modifiers may be provided as part of the geometry data in block ELEME. This feature can be used to apply spatially correlated fields: users can run their own geostatistical package to generate whatever fields they desire, and then use a preprocessing program to place the PM-coefficients into the ELEME data block.

Note that this approach of block-by-block permeability modification affects only grid block permeabilities but not porosities. Full details on the various user options for PMcoefficients are given in informative printout that is automatically generated with each TOUGH+ run. 
In addition to this approach, it is possible for the users to apply a geostatistical package of their choice to develop element-specific (block-by-block) statistically heterogeneous porosities $\phi$, intrinsic permeabilities $k$, and phase saturations. These data can then be read as part of the INCON data block, as will be discussed in Section 8 . 


\subsection{Geometrical Representation, Domain Discretization, and Grid Generation}

The data blocks that specify the geometrical representation of the hydrate system are discussed in this section, including the specification of elements (block ELEM), connections between elements (block CONNE), and the generation of grids (block MESHM) for radial symmetric grids, rectilinear grids, and grids containing fractured media. First, we discuss the convention used in TOUGH + for entering and processing geometrical data.

\subsection{TOUGH+ Convention for Geometrical Data}

Handling of flow geometry data in TOUGH+ is upward compatible with TOUGH2 and TOUGH input formats and data handling. As in other integral finite difference codes [Edwards, 1972; Narasimhan and Witherspoon, 1976], flow geometry is defined by 
means of a list of volume elements ( rid blocks), and a list of flow connections between them (Section 3.3). This formulation can handle regular and irregular flow geometries in one, two, and three dimensions. Single- and multiple-porosity systems (porous and fractured media) can be specified, and higher order methods, such as seven- and ninepoint differencing, can be implemented by means of appropriate specification of geometric data [Pruess and Bodvarsson, 1983].

In TOUGH+, as in TOUGH2, volume elements are identified by names that consist of a string of either five or eight characters, '12345' or ' 12345678 '. These are arbitrary, except that the last two characters (\#4 and 5) must be numbers; an example of a valid 5character element name is 'ELE10', and an example of a valid 8-character name is 'ABOOCC23'. Flow connections are specified as ordered pairs of elements, such as ('ELE10', 'ELEII'). A variety of options and facilities are available for entering and processing geometric data. As in TOUGH2, element volumes and domain identification can be provided by means of a data block ELEME in the INPUT file, while a data block CONNE can be used to supply connection data, including interface area, nodal distances from the interface, and orientation of the nodal line relative to the vertical (see Sections 7.2 and 7.3). These data are internally written to a disk file MESH, which in turn initializes the geometry data arrays used during the flow simulation. It is also possible to omit the ELEME and CONNE data blocks from the INPUT file, and provide geometry data directly on a disk file MESH.

TOUGH + provides a capability for describing the flow system geometry and discretizing the domain by means of the MeshMaker.f95 application written in a FORTRAN95/2003 (see Section 7.4). Unlike the approach in the TOUGH [Pruess, 
1987] and TOUGH2 [Pruess, 1991a; Pruess et al., 1999] codes, MeshMaker.f95 is separate from (i.e., it is not integrated within) the TOUGH+ code. This application can perform a number of mesh generation and processing operations. The MeshMaker . f 95 code is written according to the tenets of Object-Oriented Programming, and has a modular structure. It can generate two-dimensional radially symmetric $(r, z)$ meshes, and one-, two-, and three-dimensional rectilinear (Cartesian) grids in $(x, y, z)$. Multipleporosity processing for simulation of flow in naturally fractured reservoirs can be invoked by means of a keyword MINC, which stands for Multiple INteracting Continua (see Section 2.14). The MINC process operates on the data of the primary (porous medium) mesh as provided on disk file MESH, and generates a secondary mesh containing fracture and matrix elements with identical data formats on file MINC. The file MESH used in this process can be either directly supplied by the user, or it can have been generated from an earlier application of the MeshMaker. $f 95$ application.

\subsection{Data Block ELEME}

This block introduces element (grid block) information.

Record ELEME. 1 (for 5-character elements only)

Format (A3, I2, 2I5, A5, 6E10.4, 1X, A1)

ElName5C, NSEQ, NADD, MA12, elem_vol, elem_aht, elem_pm, $\mathrm{X}, \mathrm{Y}, \mathrm{Z}, \mathrm{elem}-\bar{a} c t i v i t y$

ElName5C

The five-character name of an element. The first three characters can be letters, numbers or blanks, and the last two characters $(\mathrm{NE}=$ ElName5C (4:5)) must be numbers. 
NSEQ

NADD

MA12

elem_vol

elem_aht

elem_pm

$X, Y, Z$
The number of additional elements having the same volume and belonging to the same reservoir domain.

The increment between the code numbers of two successive elements. (Note: if the longest dimension in the grid is $<1000$, the maximum permissible code number NE + NSEQ *NADD is $\leq$ 999.)

A five-character material identifier corresponding to one of the reservoir domains as specified in block ROCKS. If the first three characters are blanks and the last two characters are numbers, then they indicate the sequence number of the domain as entered in ROCKS. If left blank the element is by default assigned to the first domain in block ROCKS.

The element volume $\left(\mathrm{m}^{3}\right)$.

The interface area $\left(\mathrm{m}^{2}\right)$ for heat exchange with semi-infinite confining beds. Internal MESH generation via the MeshMaker. $\mathbf{f} 95$ facility will automatically generate elem_aht.

The permeability modifier (optional, active only when a domain "SEED" has been specified in the ROCKS block; see Section 6.5). Will be used as multiplicative factor for the permeability parameters from block ROCKS. Simultaneously, the capillary pressure strength will be scaled as elem_pm ${ }^{-1 / 2}$. elem_pm $=0$ will result in an impermeable block.

Random permeability modifiers can be generated internally, see detailed comments in the TOUGH+ output file. The elem_pm may be used to specify spatially correlated heterogeneous fields, but users need their own preprocessing programs for this, as $\mathrm{TOUGH}+$ provides no internal capabilities for generating such fields.

Cartesian coordinates of the grid block centers. These are included in the ELEME data for listing in output files of the simulation results that conform to the specifications of the TecPlot package. Additionally, the coordinate data are be needed for 
elem_activity (a) the computation of interblock properties in cylindrical grids, (b) the initialization of a gravitycapillary equilibrium (such as the one described in Sections 6.9 and 9.10 of Pruess et al. [1999]), and (c) for the computation of quantities related to mechanical dispersion in solute transport investigations [Moridis et al., 1999].

A (optional) character variable that describes the activity status of an element. An element is rendered inactive (i.e., acting as a Dirichlet boundary) by invoking the options elem_activity $=I$ (indicating that the element conditions are time-invariant) or elem_activity $=\mathrm{V}$ (denoting time-variable conditions). The grid block is assumed active by default for any other value of elem_activity, including a blank (see Section 8.4).

Record ELEME. 1 (for 8-character elements only)

$$
\begin{aligned}
& \text { Format (A8, 7X, A5, 6E10.4, 1X, A1) } \\
& \text { ElName8C, MA12, } \\
& \text { elem_vol, elem_aht, elem_pm, } \\
& \text { X, Y, Z, elem_activity }
\end{aligned}
$$

ElName8C

The eight-character name of an element. The first five characters can be letters, numbers or blanks, and the last two characters $(\mathrm{NE}=$ ElName 8C ( $7: 8))$ must be numbers.

All other variables and parameters are as previously defined in case of the fivecharacter element names.

Repeat record ELEME. 1 for the number of elements desired.

Record ELEME. 2 A blank record closes the ELEME data block.

NOTE: The number of elements described in the ELEME data block cannot exceed the number Max_NumElem specified in the MEMORY data block (See 
Section 5.1). If this happens, an error message is printed and the simulation is aborted.

\subsection{Data Block CONNE}

This block introduces information for the connections (interfaces) between elements.

Record CONNE. 1 (for connections involving 5-character elements only)

Format (A5, A5, 4I5, 5E10.4)

ConxName1, ConxName2, NSEQ, NAD1, NAD2, ConxKi, ConxD1, ConxD2, ConxArea, ConxBeta, emissivity

ConxName1

ConxName2

NSEQ

NAD1

NAD2

ConxKi
The name of the first five-character element in the connection.

The code name of the five-character second element in the connection.

The number of additional connections in the sequence.

The increment of the number of the first element (as defined by the last two characters of its name, i.e., ConxName1 (4:5)) between two successive connections.

The increment of number of the second element (as defined by the last two characters of its name, i.e., ConxName2 (4:5)) between two successive connections.

The permeability index of the connection. Setting it equal to 1,2 , or 3 specifies absolute permeability to be MediaPerm (ConxKi) for the materials in elements (ConxName1) and (ConxName2), where MediaPerm is read in block ROCKS. This allows assignment of different permeabilities, e.g., in the horizontal and vertical direction. 
ConxD1, ConxD2

ConxArea

ConxBeta

emissivity
The distance $(\mathrm{m})$ between the common interface of a connection from the centers of the first and second element in the connection, respectively.

The element interface area $\left(\mathrm{m}^{2}\right)$.

The cosine of the angle between the gravitational acceleration vector and the line between the two elements. ConxBeta*gravity $>0 \quad(<0)$ corresponds to first element being above (below) the second element.

The radiant emittance factor for radiative heat transfer, which for a perfectly "black" body is equal to 1 . The rate of radiative heat transfer between the two grid blocks is:

$$
Q_{H, r a d}=\text { emissivity } * \sigma_{0} * \text { ConxArea } *\left(T_{2}^{4}-T_{1}^{4}\right)
$$

where $\sigma_{0}=5.6687 \mathrm{e}-8 \mathrm{~J} / \mathrm{m}^{2} \mathrm{~K}^{4} \mathrm{~s}$ is the StefanBoltzmann constant, and $T_{1}$ and $T_{2}$ are the absolute temperatures of the two grid blocks. The term emissivity may be entered as a negative number, in which case the absolute value will be used, and heat conduction at the connection will be suppressed. Setting emissivity $=0.0$ e 0 will result in no radiative heat transfer.

$\underline{\text { Record CONNE . } 1 \text { (for connections involving 5-character elements only) }}$

Format (A8, A8, 9X, I5, 5E10.4)

Conx8Name1, Conx8Name2, ConxKi, ConxD1, ConxD2, ConxArea, ConxBeta, emissivity

Conx8Name1

Conx8Name2

$\mathrm{NAD} 1$
The name of the first eight-character element in the connection.

The code name of the second eight-character element in the connection.

The increment of the number of the first element (as defined by the last two characters of its name, i.e., ConxName1 $(7: 8)$ ) between two successive connections. 
$\mathrm{NAD} 2$

The increment of number of the second element (as defined by the last two characters of its name, i.e., ConxName2 $(8: 8)$ ) between two successive connections.

All other variables and parameters are as previously defined in case of connections between five-character elements.

Repeat record CONNE . 1 for the number of connections desired.

Record CONNE . 2 A blank record closes the CONNE data block. Alternatively, connection information may terminate on a record with ' $+++\quad$ ' typed in the first five columns, followed by element crossreferencing information. The second type of termination is generated in the MESH file upon completion of a $\mathrm{TOUGH}+$ run.

NOTE: The number of connections described in the CONNE data block cannot exceed the number Max_NumCOnX specified in the MEMORY data block (See Section 5.1). If this happens, an error message is printed and the simulation is aborted

\subsection{The MeshMaker . f95 Facility}

In this section we discuss the use of the MeshMaker. $f 95$ facility, and the required parameter inputs for mesh generation and processing. As indicated earlier, unlike in TOUGH2 [Pruess et al., 1999], MeshMaker. $f 95$ is not integrated into the TOUGH+ code but is an independent program that is written in FORTRAN 95/2003, has a modular structure, and an architecture based on the principles of Object-Oriented Programming. The MeshMaker. $\mathbf{f 9 5}$ input has a modular structure, which is organized by keywords in a manner analogous to that of TOUGH+. This section provides detailed instructions for preparing the input files, illustrative examples of which are shown in Figures 7.1 to 7.4. 


\subsubsection{Inputs Related to Problem Definition and Dimensioning}

These inputs occupy two records (both mandatory), and provide (a) a short description of the problem through an informative title, and (b) data to allow proper dimensioning of the work arrays and formatting of the MESH file to be generated. These two initial records are discussed in detail below:

\section{Record MESHMAKER . 1}

The first record of the input file in any MeshMaker. $\mathbf{f 9 5}$ application is the character variable TITLE, which includes a header of up to 80 characters and is read using a free format. This record is necessary for any MeshMaker simulation to begin.

\section{Record MESHMAKER . 2}

The following variables are read in MESHMAKER . 2 using a free format:

$$
\text { MaxNum_Elem, Longest, ElemNameLength, FormatType }
$$

These parameters are defined as follows:

Max_NumElem Integer denoting the maximum number of elements in the grid under construction.

Longest Integer indicating the maximum expected number of subdivisions along any of the coordinates in the grid under construction.

ElemNameLength Integer variable defining the number of characters in the element names. It may be either 5 or 8 . If unequal to 8, ElemNameLength is internally resest to the default $(=5)$.

Format Type

Character variable indicating the format of the data in the MESH file to be created. It my have one of two values:

= 'Old': This option creates a MESH file that conforms to the data format described in Sections 7.2 and 7.3, and is consistent with the TOUGH 
[Pruess, 1987] and TOUGH2 formats [Pruess, 1991; Pruess et al., 1999].

= 'New': This option creates a MESH file in which the element and connection data are listed using the NAMELIST format facility available in FORTRAN $90 / 95 / 2003$. Inputs in the next generation of TOUGH+ (currently in development) will make extensive use of NAMELIST-based formats.

NOTE: The current version (v1.0) of TOUGH+ and $T+H$ does not accept NAMELIST-based formats in the MESH file. Thus, the option FormatType = 'Old' must be used in all MeshMaker.f95 applications.

\subsubsection{Inputs Related to Domain Heterogeneity}

These optional inputs provide information that allows the description of heterogeneity within the domain. The assignment of heterogeneity is based on the definition of regions using geometrical information that describe the location and extent of these subdomains. Thus, each of the individually defined regions is assigned the properties of a particular medium that will have to be included in the ROcKS data block in the input file of the subsequent $\mathrm{TOUGH}+$ simulation.

The records in this data block are discussed in detail below:

\section{Record MESHMAKER . 3 (Optional)}

This record includes only the keyword (character variable) 'Regions', which is read using a free format. If the domain is homogeneous, then there is no need to provide any of the inputs discussed in Section 7.4.2. 
Record MESHMAKER . 3.1 (Optional - when the MESHMAKER . 3 record is included)

This record is read using a free format, and includes the single integer variable Num_HetRegions $(>0)$ that describes the number of heterogeneous regions (subdomains) that are to be described by the ensuing data records. The minimum value that Num_HetRegions can accept is 1, corresponding to a homogeneous system. An error will occur and the execution will stop if Num_HetRegions < 1.

Because of dynamic dimensioning, there is no limit in the number of heterogeneous regions defined by Num_HetRegions. However, practical considerations may limit the size of Num_HetRegions. Thus, although it is possible to define element-by-element heterogeneity using this approach, this would be a very tedious process. Generally speaking, the most useful application of this facility is in the description of extensive geologic units with distinctly different properties.

Record MESHMAKER. 3.2 (Optional -when the MESHMAKER . 3 record is included)

This record is read using a free format, and includes the single character variable dominant medium that provides the name (5 character long) of the dominant (reference) porous medium in the heterogeneous domain. Note that the selection of a medium as dominant medium is be arbitrary, as there are no restrictions on the extent of its spatial distribution for it to be designated as such.

If Num_HetRegions $=1$, no more data need to be read. This case is equivalent to a homogeneous system, and the entire heterogeneity-related data block (records MESHMAKER . 3 to MESHMAKER . 3.2 may be omitted.

Record MESHMAKER . 3.3.0 (Optional - when the MESHMAKER . 3 record is included and Num_HetRegions $>1$ )

This record is read using a free format, and includes the single character variable H_RegionNname that provides the name (5 character long) of the porous medium in the region (subdomain) that is about to be defined.

Record MESHMAKER. 3.3.1 (Optional - when the MESHMAKER . 3 record is included and Num HetRegions $>1$ )

The following character variables are read in MESHMAKER.3.3.1 using a free format: 


$$
\text { H_RegionCoordinates, H_RegionUnits }
$$

These parameters are defined as follows:

$\mathrm{H}$-RegionCoordinates A character variable indicating the coordinate system used in the geometric definition of the region that is about to be described in the heterogeneous domain. It my have one of two values:

$=$ 'Cartesian': This option indicates that the region is defined geometrically in terms of Cartesian coordinates.

$=$ 'Cylindrical': This option indicates that the region is defined geometrically in terms of cylindrical coordinates.

H_RegionUnits

A character variable describing the units of length used in the description of the geometry of the region. The following values are acceptable options:

'mm', 'km', 'km', 'in', or 'ft', indicating millimeters, meters, kilometers, inches and feet, respectively. Note that MeshMaker. $f 95$ converts all length units into meters (the length unit used in the TOUGH+ simulations) prior to producing the MESH file.

Record MESHMAKER. 3.3.2 (Optional - when the MESHMAKER. 3 record is included and Num HetRegions $>1$ )

If $\mathrm{H}$ RegionCoordinates = 'Cartesian', the following real variables are read in MESHMAKER . 3 . 3 2 using a free format:

$$
\text { Xmin, Xmax, Ymin, Ymax, Zmin, Zmax }
$$

These parameters are defined as follows:
$\mathrm{Xmin}, \mathrm{Xmax}$
Real variables indicating the range of the region along the $x$-axis of the Cartesian coordinate system.
Ymin, Ymax
Real variables indicating the range of the region along the $y$-axis of the Cartesian coordinate system. 
Zmin, Zmax

Real variables indicating the range of the region along the $z$-axis of the Cartesian coordinate system.

If $H_{-}$RegionCoordinates = 'Cylindrical', the following real variables are read in MESHMAKER . 3.3.2 using a free format:

$$
\text { Rmin, Rmax, Zmin, Zmax }
$$

These parameters are defined as follows:

$$
\begin{aligned}
& \text { Rmin, Rmax } \begin{array}{l}
\text { Real variables indicating the range of the region } \\
\text { along the } r \text {-axis of the cylindrical coordinate } \\
\text { system. }
\end{array} \\
& \text { Zmin, Zmax } \quad \begin{array}{l}
\text { Real variables indicating the range of the region } \\
\text { along the } z \text {-axis of the cylindrical coordinate } \\
\text { system. }
\end{array}
\end{aligned}
$$

Repeat records MESHMAKER.3.3.0, MESHMAKER.3.3.1, MESHMAKER. 3.3 .3 for a total of Num_HetRegions -1 regions. Note that the region dominant_medium does not need geometric definition.

\subsubsection{Inputs Related to Description of Boundaries}

These optional inputs provide information that describes the outer boundaries of the domain under discretization. The assignment of grid subdomains as boundaries is based on the geometry-based definition of the outer spatial limits of the domain under discretization. Thus, the boundaries are treated as special types of regions (see Section 7.4.2) with the properties of a particular medium that will have to be included in the ROCKS data block in the input file of the subsequent TOUGH+ simulation. It is possible for a region and one or more boundaries to be described by the same medium in the ROCKS data block.

The records in this data block are discussed in detail below:

\section{Record MESHMAKER . 4 (Optional)}


This record includes only the keyword (character variable) 'Boundaries', which is read using a free format. If the domain is confined by no-flow (Newman-type) boundaries, then there is no need to provide any of the inputs discussed in Section 7.4.3.

Record MESHMAKER . 4 .1 (Optional - when the MESHMAKER . 4 record is included)

This record is read using a free format, and includes the single integer variable Num_Boundaries $(>0)$ that describes the number of boundaries that are to be described.

Record MESHMAKER . 4.2 .0 (Optional - when the MESHMAKER . 4 record is included and Num Boundaries $>1$ )

The following character variables are read in MESHMAKER.4.2 using a free format:

$$
\text { BoundID, BoundRegionName }
$$

These parameters are defined as follows:

BoundID

BoundRegionName
A character variable indicating the type of boundary described at the prescribed location. It my have one of two values:

= 'I': This option indicates an inactive boundary, the conditions and properties of which are timeinvariant.

$={ }^{\prime} \mathrm{V}$ ': This option indicates a time-variable boundary.

The result of this designation is reflected in the ELEME block, in which elem_activity (see Section 7.2) is set to BoundID in all the cells corresponding to the boundary defined here. Any other value of the BoundID variable causes the program to print an error message and stop execution.

A character variable $\mathrm{H} \_$RegionNname that provides the name ( 5 character long) of the porous medium in the boundary that is about to be defined. 
Record MESHMAKER .4.2.1 (Optional-when the MESHMAKER . 4 record is included and Num Boundaries $>1$ )

The following character variables are read in MESHMAKER.4.2.1 using a free format:

$$
\text { BoundRegionCoordinates, BoundRegionUnits }
$$

These parameters are defined as follows:

BoundRegionCoordinates A character variable indicating the coordinate system used in the geometric definition of the boundary that is about to be described in the heterogeneous domain. It my have one of two values:

$=$ 'Cartesian': This option indicates that the region is defined geometrically in terms of Cartesian coordinates.

$=$ 'Cylindrical': This option indicates that the region is defined geometrically in terms of cylindrical coordinates.

BoundRegionUnits A character variable describing the units of length used in the description of the geometry of the boundary. The following values are acceptable options:

'mm', 'km', 'km', 'in', or 'ft',

indicating millimeters, meters, kilometers, inches and feet, respectively. Note that

MeshMaker. $f 95$ converts all length units into meters (the length unit used in the TOUGH+ simulations) prior to producing the MESH file. 
Record MESHMAKER .4.2.2 (Optional-when the MESHMAKER . 4 record is included and Num Boundaries $>1$ )

If BoundRegionCoordinates = 'Cartesian', the following real variables are read in MESHMAKER.3.3.2 using a free format:

$$
\text { Xmin, Xmax, Ymin, Ymax, Zmin, Zmax }
$$

These parameters are defined as follows:

$\mathrm{xmin}, \mathrm{Xmax}$

Real variables indicating the range of the boundary region along the $x$-axis of the Cartesian coordinate system.

Ymin, Ymax Real variables indicating the range of the boundary region along the $y$-axis of the Cartesian coordinate system.

Zmin, Zmax Real variables indicating the range of the boundary region along the $z$-axis of the Cartesian coordinate system.

If BoundRegionCoordinates = 'Cylindrical', the following real variables are read in MESHMAKER. 3 . 3 .2 using a free format:

$$
\text { Rmin, Rmax, Zmin, Zmax }
$$

These parameters are defined as follows:

Rmin, Rmax Real variables indicating the range of the boundary region along the $r$-axis of the cylindrical coordinate system.

Zmin, Zmax Real variables indicating the range of the boundary region along the $z$-axis of the cylindrical coordinate system.

Repeat records MESHMAKER . 4.2 .0, MESHMAKER .4.2 .1,

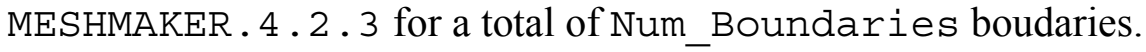

\subsubsection{Inputs for Grid Construction}

There are three grid construction options available in MeshMaker. $f 95$. These options are activated by appropriate keywords. Thus, the keywords 'RZ2D' or 'RZ2DL' 
invoke generation of a one or two-dimensional radially symmetric $(r, z)$ mesh; 'XYZ' initiates generation of a one, two, or three dimensional Cartesian $(x, y, z)$ mesh; and 'MINC' calls a modified version of the GMINC program [Pruess, 1983] to sub-partition a primary porous medium mesh into a secondary mesh for fractured media, using the method of Multiple INteracting Continua [Pruess and Narasimhan, 1982; 1985].

The meshes generated under keyword 'RZ2D' or 'XYZ' are internally written to file MESH. The 'MINC' processing operates on the data in file MESH, so that invoking the 'RZ2D' or 'XYZ' options, or assignment of ELEME and CONNE blocks in the INPUT file, must precede the MeshMaker.f95/MINC data. We shall now separately describe the preparation of input data for the three grid construction options.

7.4.4.1. Generation of radially symmetric grids (keyword 'RZ2D' or 'RZ2DL'). The keywords 'RZ2D' or 'RZ2DL' invoke generation of a radially symmetric mesh. Values for the radii to which the grid blocks extend can be provided by the user or can be generated internally (see below). Nodal points will be placed halfway between neighboring radial interfaces. When 'RZ2D' is specified, the mesh will be generated by columns; i.e., in the ELEME block we will first have the grid blocks at smallest radius for all layers, then the next largest radius for all layers, and so on.

With keyword 'RZ2DL' the mesh will be generated by layers; i.e., in the ELEME block we will first have all grid blocks for the first (top) layer from smallest to largest radius, then all grid blocks for the second layer, and so on. Apart from the different ordering of elements, the two meshes for 'RZ2D' and 'RZ2DL' are identical. The reason for providing the two alternatives is as a convenience to users in implementing boundary 
conditions by way of inactive elements (see Section 6.4 in Pruess et al. [1991]). Assignment of inactive elements would be made by using a text editor on the RZ2Dgenerated MESH file, and moving groups of elements towards the end of the ELEME block, past a dummy element with zero volume. 'RZ2D' makes it easy to declare a vertical column inactive, facilitating assignment of boundary conditions in the vertical, such as a gravitationally equilibrated pressure gradient. 'RZ2DL' on the other hand facilitates implementation of areal (top and bottom layer) boundary conditions.

The inputs for cylindrical systems are as follows:

\section{Data Block GRID}

The first record in this data block includes only the keyword (character variable) 'RZ2D' or 'RZ2DL'. This keyword is read using a Format (A5).

\section{$\underline{\text { Record RADII.0 }}$}

RADI I

$\frac{\text { Record RADII . } 1}{\text { Format (I5) }}$

NRAD
A keyword that introduces data for defining a set of interfaces (grid block boundaries) in the radial direction. It is read using a Format (A5).

Record RADII . 2, RADII . 3, etc.

Format (8E10.4)

$\mathrm{RC}(i), i=1, \mathrm{NRAD}$

$\mathrm{RC}(\mathrm{i})$

Record EQUID. 0
Number of radii that will be read. At least one radius must be provided, indicating the inner boundary of the mesh. 
EQUIDistant

Keyword indicating that the ensuing data describe a set of equal radial increments. This keyword is read using Format (A5).

Record EQUID. 1

Format (I5, 5X, E10.4)

NEQU, DR

NEQU

$\mathrm{DR}$

$\underline{\text { Record LOGAR . } 0}$

LOGARithmic
The number of desired radial increments.

The size of the radial increment [m].

NOTE: At least one radius must have been defined via block RADI I before EQUID can be invoked.
This keyword introduces data on radial increments that increase from one to the next by the same factor (i.e., $\Delta r_{n+1}=f \cdot \Delta r_{n}$ ).

Record LOGAR . 1

Format(I5, 5X, 2E10.4)

NLOG， RLOG，DR

NLOG

RLOG

$\mathrm{DR}$
The number of desired radial increments.

The desired radius $r_{\max }$ of the last (largest) of these radii.

The reference radial increment $\Delta r_{0}$ : the first $\Delta r$ generated will be equal to $f \cdot \Delta r_{0}$, with $f$ internally determined such that the last increment will bring total radius to $\mathrm{RLOG}=r_{\max }$. The factor $f<1$ for decreasing radial increments is permissible. If $\Delta r_{0}$ is set equal to zero, or left blank, the last increment $\Delta r$ generated before the keyword LOGAR is invoked will be used as default.

Additional blocks RADII, EQUID, and LOGAR can be specified in arbitrary order.

NOTE: At least one radius must have been defined before the LOGAR option can be invoked. If $\Delta r_{0}=0$, at least two radii must have been defined. 
Record LAYER. 0

LAYER This keyword introduces information on horizontal layers, and signals closure of RZ2D input data. It is read using a Format (A5).

Record LAYER. I

Format (I5)

NLAY

NLAY The number of horizontal layers in the cylindrical grid.

Record LAYER . 2

Format (8E10.4)

$\mathrm{H}(i), i=1, \mathrm{NLAY}$

$\mathrm{H}$ (i) The thicknesses of the horizontal layers in the cylindrical grid, from top layer downward. By default, zero or blank entries for layer thickness will result in assignment of the last preceding nonzero entry. Assignment of a zero layer thickness, as needed for inactive layers, can be accomplished by specifying a negative value.

NOTE: The LAYER data close the RZ2D or RZ2DL data block. Note that one blank record must follow to indicate termination of the GRID data block. Alternatively, the keyword 'MINC' can appear to invoke MINC-processing for fractured media (see below).

\subsubsection{Generation of rectilinear grids (keyword ' $X Y Z$ ')}

\section{Data Block GRID}

The first record in this data block includes only the keyword (character variable) 'XYZ' that is invokes generation of a Cartesian (rectilinear) mesh. This keyword is read using a Format (A5). 
Record XYZ. 1

Format (E10.4)

DEG

DEG

The angle (in degrees) between the $y$-axis and the horizontal. If gravitational acceleration (parameter gravity in record PARAM.2, see Section 10) is specified positive, $-90^{\circ}<\mathrm{DEG}<90^{\circ}$ corresponds to grid layers going from top down. Grids can be specified from the bottom layer up by setting gravity or ConxBeta (Section 7.3) to negative values. The default $(\mathrm{DEG}=0.0 \mathrm{e} 0)$ corresponds to horizontal $y$-and vertical $z$-axis. The $x$-axis is always horizontal.

Record XYZ.2

Format (A2, 3X, I5, E10.4)

NTYPE, NO, DEL

NTYPE

A character variable that can assume one of the values ' $\mathrm{NX}$ ', 'NY' or 'NZ', specifying grid increments in the $x$-, $y$-, or $z$-direction, respectively.

NO

The number of grid increments.

DEL

The constant grid increment for NO grid blocks, if set to a non-zero value.

$\underline{\text { Record XYZ.3 (Optional, DEL }=0.0 \mathrm{e} 0 \text { or blank only) }}$

Format (8E10.4)

$\operatorname{DEL}(i), i=1, \mathrm{NO}$

DEL (i)

A set of grid increments in the direction specified by NTYPE in record XYZ.2. Additional records with formats as XYZ. 2 and XYZ. 3 can be provided, with $x$-, $y$-, and $z$-data in arbitrary order.

Record XYZ.4 A blank record closes the XYZ data block.

NOTE: The end of the data block GRID is also marked by a blank record. Thus, when GRID/XYZ is used, there will be two blank records at the end of the corresponding input data block. 


\subsubsection{MINC processing for fractured media (keyword 'MINC')}

\section{Data Block GRID}

The first record in this data block includes only the keyword (character variable) 'MINC' that is invokes post-processing of a primary porous medium mesh from a previously developed file MESH. This keyword is read using a Format (A5). The input formats in data block MINC are identical to those of the GMINC program [Pruess, 1983], with two enhancements: (a) there is an additional facility for specifying global matrix-matrix connections (dual permeability option); (b) only active elements (see Section 8.4) will be subjected to MINC-processing, the remainder of the MESH remaining unaltered as porous medium grid blocks. See Section 2.14 for further discussion.

$\underline{\text { Record MINC. } 1}$

Format (2A5, 5X, A5)

PART, TYPE, DUAL

PART

PART

TYPE
This is the first keyword following the 'MINC' keyword. It will be followed on the same line by parameters TYPE and DUAL with information on the nature of fracture distributions and matrixmatrix connections.

An identifier of the data block with partitioning parameters for secondary mesh.

A five-character variable for selecting one of the following six different proximity functions provided in MINC [Pruess, 1983].

$=$ 'ONE-D': A set of plane parallel infinite fractures with matrix block thickness between neighboring fractures equal to PAR ( 1 ).

$=$ 'TWO $-\mathrm{D}$ ': Two sets of plane parallel infinite fractures, with arbitrary angle between them. Matrix block thickness is PAR ( 1 ) for the first set, and $\operatorname{PAR}(2)$ for the second set. If PAR (2) is not specified explicitly, it will be set equal to PAR ( I ). 
$=$ 'THRED 1 : Three sets of plane parallel infinite fractures at right angles, with matrix block dimensions of PAR (1), PAR (2), and PAR (3), respectively. If PAR (2) and/or PAR (3) are not explicitly specified, they will be set equal to PAR (1) and/or PAR (2), respectively.

$=$ 'STANA $'$ : Average proximity function for rock loading of Stanford large reservoir model [Lam et al., 1988].

$=$ 'STANB ' : Proximity function for the five bottom layers of Stanford large reservoir model.

$=$ 'STANT ' $:$ Proximity function for top layer of Stanford large reservoir model.

DUAL

A five-character word for selecting the treatment of global matrix flow.

$=1 \quad$ (Blank - default): The global flow occurs only through the fracture continuum, while rock matrix and fractures interact locally by means of interporosity flow (double-porosity model).

$=$ 'MMVER': The global matrix-matrix flow is permitted only in the vertical; otherwise like the double-porosity model; for internal consistency this choice should only be made for flow systems with one or two predominantly vertical fracture sets.

= 'MMALL 1 : The global matrix-matrix flow in all directions; for internal consistency only two continua, representing matrix and fractures, should be specified (dual-permeability model).

NOTE: A user wishing to employ a different proximity function other than the options provided through the TYPE variable in MINC needs to replace the

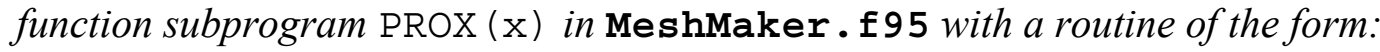

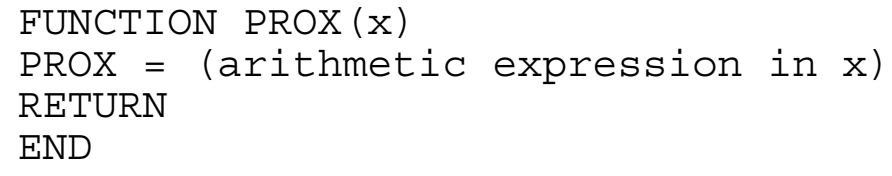


It is necessary that $\mathrm{PROX}(\mathrm{x})$ be defined even when $x$ exceeds the maximum possible distance from the fractures, in which case PROX $=1$. Additionally, when the users supply their own proximity function subprogram, the parameter TYPE must be set equal to ' ONE-D ', 'TWO-D ', or 'THRED', depending on the dimensionality of the proximity function. This will assure proper definition of innermost nodal distance [Pruess, 1983].

Record PART. 1

Format (2I3, A4, 7E10.4)

J, NVOL, WHERE, (PAR(i), $i=1,7)$

$\mathrm{J}$

NVOL

WHERE

$\operatorname{PAR}(i)$
The total number of multiple interacting continua ( $\mathrm{J}<36)$.

The total number of explicitly provided volume fractions (NVOL $<J$ ). If NVOL $<J$, the volume fractions with indices NVOL $+1, \ldots, \mathrm{J}$ will be internally generated; all being equal and chosen such as to yield proper normalization to 1 .

Character variable specifying whether the sequentially specified volume fractions begin with the fractures (WHERE = 'OUT' $)$ or in the interior of the matrix blocks (WHERE = 'IN').

Real array that stores the parameters describing the fracture spacing (see discussion of previous record).

Record PART.2.1, PART.2.2, etc.

Format (8E10.4)

(VOL (i), $i=1$, NVOL)

VOL (i)

The volume fraction (having a value between 0 and 1) of a continuum with index $i$ (for WHERE = ' OUT') or index J+l-i (for WHERE = 'IN'). NVOL volume fractions will be read. For WHERE $=$ ' OUT ', $i=1$ is the fracture continuum, $i=2$ is the matrix continuum closest to the fractures, $i=3$ is the matrix continuum adjacent to $i=2$, etc. The sum of all volume fractions must not exceed 1 . 


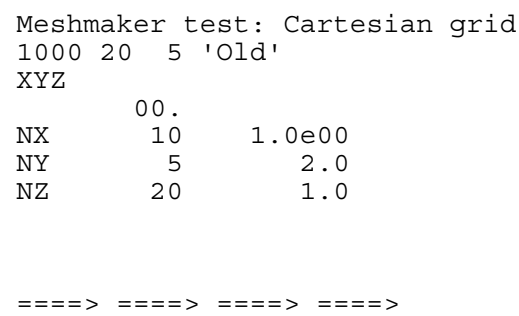

Figure 7.1. An example of a MeshMaker. $\mathbf{9} 95$ input file for the creation of a Cartesian 3D grid. Note that no heterogeneous regions or boundaries are defined in this grid.

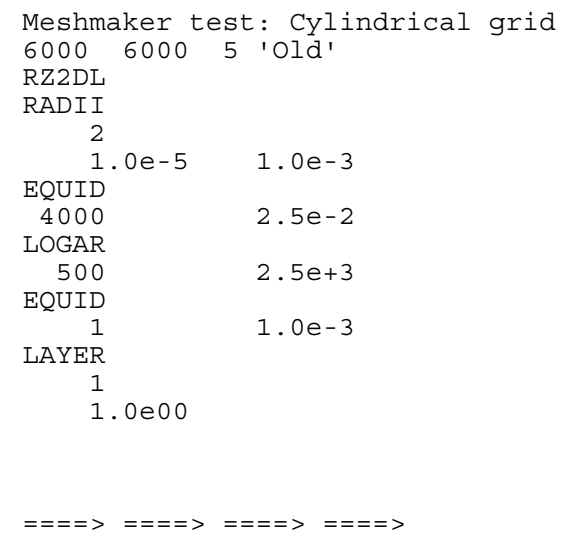

Figure 7.2. An example of a MeshMaker. $f 95$ input file for the creation of a single-layer (1D) cylindrical grid. Note that no heterogeneous regions or boundaries are defined in this grid. 
Input file for a large 3D cartesian grid 5000001005 'Old'

Format Type

Regions

' HydrL'

'Aquif'

'cartesian' 'm'

$0.0 e 01.5 e 3 \quad 0.0 e 0 \quad 1.5 e 3-6.3 e 1-4.825 e 1$ 'OverB'

'cartesian' 'm'

$0.0 \mathrm{e} 01.5 \mathrm{e} 3 \quad 0.0 \mathrm{e} 0 \quad 1.5 \mathrm{e} 3 \quad-3.0 \mathrm{e} 1 \quad 0.0 \mathrm{e} 1$

'UndrB'

'cartesian' 'm'

$\begin{array}{llllll}0.0 e 0 & 1.5 e 3 & 0.0 e 0 & 1.5 e 3 & -9.4 e 1 & -6.3 e 1\end{array}$

'WellA'

'cartesian' 'm'

$0.0 e 0 \quad 5.0 e-2 \quad 0.0 e 0 \quad 5.0 e-2-5.4 e 1-3.0 e 1$

'WellA'

'cartesian' 'm'

4.9995e2 5.0e2 4.9995e2 5.0e2 -5.4 e1 $-3.0 e 1$

Boundaries

'I' 'TopBB'

'cartesian' 'm'

$0.0 e 0 \quad 1.5 e 3 \quad 0.0 e 0 \quad 1.5 e 3-1.0 e-2 \quad 0.0 e 0$

'I' 'BotBB'

'cartesian' 'm'

$0.0 \mathrm{e} \quad 1.5 \mathrm{e} 30.0 \mathrm{e} 01.5 \mathrm{e} 3-9.4 \mathrm{e} 1-9.3 \mathrm{e} 1$

$\mathrm{XYZ}$

NX

00 .
! Title

! MaxNum Elem, Longest, ElemNameLength,

! ==> \# of heterogeneous media regions

! Region \#1: Name of dominant medium

Region \#2: medium name coordinates, units

Xmin, Xmax, Ymin, Ymax, Zmin, Zmax

Region \#3: medium name

coordinates, units

Rmin, Rmax, Zmin, Zmax

Region \#4: medium name coordinates, units Rmin, Rmax, Zmin, Zmax

Region \#5: medium name coordinates, units Rmin, Rmax, Zmin, Zmax

Region \#6: medium name

coordinates, units

Rmin, Rmax, Zmin, Zmax

$==>$ \# of boundaries

Boundary \#1: type and medium name coordinates, units Rmin, Rmax, Zmin, Zmax

Boundary \#2: type and medium name coordinates, units

Rmin, Rmax, Zmin, Zmax

$5.0 e-2 \quad 2.5000 e-1 \quad 2.8599 e-1 \quad 3.2716 e-1 \quad 3.7426 e-1 \quad 4.2813 e-1 \quad 4.8976 e-1 \quad 5.6027 e-1$ $6.4092 e-1 \quad 7.3319 e-1 \quad 8.3873 e-1 \quad 9.5947 e-1 \quad 1.0976 e+0 \quad 1.2556 e+0 \quad 1.4363 e+0 \quad 1.6431 e+0$ $\begin{array}{llllllll}1.8797 e+0 & 2.1502 e+0 & 2.4598 e+0 & 2.8139 e+0 & 3.2190 e+0 & 3.6823 e+0 & 4.2124 e+0 & 4.8188 e+0\end{array}$

$5.5125 \mathrm{e}+0 \quad 6.3061 \mathrm{e}+0 \quad 7.2139 \mathrm{e}+0 \quad 8.2524 \mathrm{e}+0 \quad 9.4404 \mathrm{e}+0 \quad 1.0799 \mathrm{e}+1 \quad 1.2354 \mathrm{e}+11.4132 \mathrm{e}+1$

$\begin{array}{llllllll}1.6167 e+1 & 1.8494 e+1 & 2.1157 e+1 & 2.4202 e+1 & 2.7686 e+1 & 3.1672 e+1 & 3.1672 e+1 & 2.7686 e+1\end{array}$

$2.4202 \mathrm{e}+1 \quad 2.1157 \mathrm{e}+1 \quad 1.8494 \mathrm{e}+1 \quad 1.6167 \mathrm{e}+1 \quad 1.4132 \mathrm{e}+1 \quad 1.2354 \mathrm{e}+1 \quad 1.0799 \mathrm{e}+1 \quad 9.4404 \mathrm{e}+0$

$\begin{array}{llllllll}8.2524 e+0 & 7.2139 e+0 & 6.3061 e+0 & 5.5125 e+0 & 4.8188 e+0 & 4.2124 e+0 & 3.6823 e+0 & 3.2190 e+0\end{array}$

$2.8139 e+02.4598 e+0 \quad 2.1502 e+01.8797 e+0 \quad 1.6431 e+01.4363 e+01.2556 e+01.0976 e+0$

$9.5947 \mathrm{e}-1 \quad 8.3873 \mathrm{e}-1 \quad 7.3319 \mathrm{e}-1 \quad 6.4092 \mathrm{e}-1 \quad 5.6027 \mathrm{e}-1 \quad 4.8976 \mathrm{e}-1 \quad 4.2813 \mathrm{e}-1 \quad 3.7426 \mathrm{e}-1$

$3.2716 \mathrm{e}-1 \quad 2.8599 \mathrm{e}-1 \quad 2.5000 \mathrm{e}-1 \quad 5.0 \mathrm{e}-2$

NY 76

5.0e-2 2.5000e-1 2.8599e-1 3.2716e-1 3.7426e-1 $4.2813 e-1 \quad 4.8976 e-1 \quad 5.6027 e-1$

$6.4092 e-1 \quad 7.3319 e-1 \quad 8.3873 e-1 \quad 9.5947 e-1 \quad 1.0976 e+0 \quad 1.2556 e+0 \quad 1.4363 e+0 \quad 1.6431 e+0$

$\begin{array}{llllllll}1.8797 e+0 & 2.1502 e+0 & 2.4598 e+0 & 2.8139 e+0 & 3.2190 e+0 & 3.6823 e+0 & 4.2124 e+0 & 4.8188 e+0\end{array}$

$5.5125 e+0 \quad 6.3061 e+0 \quad 7.2139 e+0 \quad 8.2524 e+0 \quad 9.4404 e+0 \quad 1.0799 e+1 \quad 1.2354 e+11.4132 e+1$

$1.6167 e+1 \quad 1.8494 e+1 \quad 2.1157 e+1 \quad 2.4202 e+1 \quad 2.7686 e+13.1672 e+13.1672 e+1 \quad 2.7686 e+1$

$2.4202 e+1 \quad 2.1157 e+1 \quad 1.8494 e+1 \quad 1.6167 e+1 \quad 1.4132 e+1 \quad 1.2354 e+1 \quad 1.0799 e+1 \quad 9.4404 e+0$

$\begin{array}{llllllll}8.2524 e+0 & 7.2139 e+0 & 6.3061 e+0 & 5.5125 e+0 & 4.8188 e+0 & 4.2124 e+0 & 3.6823 e+0 & 3.2190 e+0\end{array}$

$2.8139 \mathrm{e}+0 \quad 2.4598 \mathrm{e}+0 \quad 2.1502 \mathrm{e}+0 \quad 1.8797 \mathrm{e}+0 \quad 1.6431 \mathrm{e}+0 \quad 1.4363 \mathrm{e}+0 \quad 1.2556 \mathrm{e}+0 \quad 1.0976 \mathrm{e}+0$

$9.5947 \mathrm{e}-1 \quad 8.3873 \mathrm{e}-1 \quad 7.3319 \mathrm{e}-1 \quad 6.4092 \mathrm{e}-1 \quad 5.6027 \mathrm{e}-1 \quad 4.8976 \mathrm{e}-1 \quad 4.2813 \mathrm{e}-1 \quad 3.7426 \mathrm{e}-1$

$3.2716 \mathrm{e}-1 \quad 2.8599 \mathrm{e}-1 \quad 2.5000 \mathrm{e}-1 \quad 5.0 \mathrm{e}-2$

$\mathrm{NZ}$

$\begin{array}{rrllllll}77 & & & & & \\ 1.0 e-3 & 7.00 e 0 & 5.00 e 0 & 4.0 e+0 & 3.2 e+0 & 2.5 e+0 & 2.0 e 00 & 1.6 e 00 \\ 1.25 e 00 & 1.0 e 00 & 8.0 e-1 & 6.5 e-1 & 5.0 e-1 & 5.0 e-1 & 4.0 e-1 & 4.0 e-1 \\ 4.0 e-1 & 4.0 e-1 & 4.0 e-1 & 4.0 e-1 & 4.0 e-1 & 4.0 e-1 & 4.0 e-1 & 4.0 e-1 \\ 4.0 e-1 & 4.0 e-1 & 4.0 e-1 & 4.0 e-1 & 4.0 e-1 & 4.0 e-1 & 4.0 e-1 & 4.0 e-1 \\ 4.0 e-1 & 4.0 e-1 & 4.5 e-1 & 4.5 e-1 & 4.5 e-1 & 4.5 e-1 & 4.5 e-1 & 4.0 e-1 \\ 4.0 e-1 & 4.0 e-1 & 4.0 e-1 & 4.0 e-1 & 4.0 e-1 & 4.0 e-1 & 4.0 e-1 & 4.0 e-1 \\ 4.0 e-1 & 4.0 e-1 & 4.0 e-1 & 4.0 e-1 & 4.0 e-1 & 4.0 e-1 & 4.0 e-1 & 4.0 e-1 \\ 4.0 e-1 & 4.0 e-1 & 4.0 e-1 & 5.0 e-1 & 5.0 e-1 & 6.5 e-1 & 8.0 e-1 & 1.0 e 00 \\ 1.0 e 00 & 1.25 e 00 & 1.6 e 00 & 2.0 e 00 & 2.5 e 00 & 3.2 e 00 & 3.0 e 00 & 4.00 e 0 \\ 4.0 e 00 & 5.0 e 00 & 6.0 e 00 & 8.0 e 00 & 1.0 e-3 & & & \end{array}$

$===>===>===>===>$

Figure 7.3. An example of a MeshMaker. $\mathbf{9} 95$ input file for the creation of a large Cartesian 3D grid with heterogeneous regions and defined boundaries. 


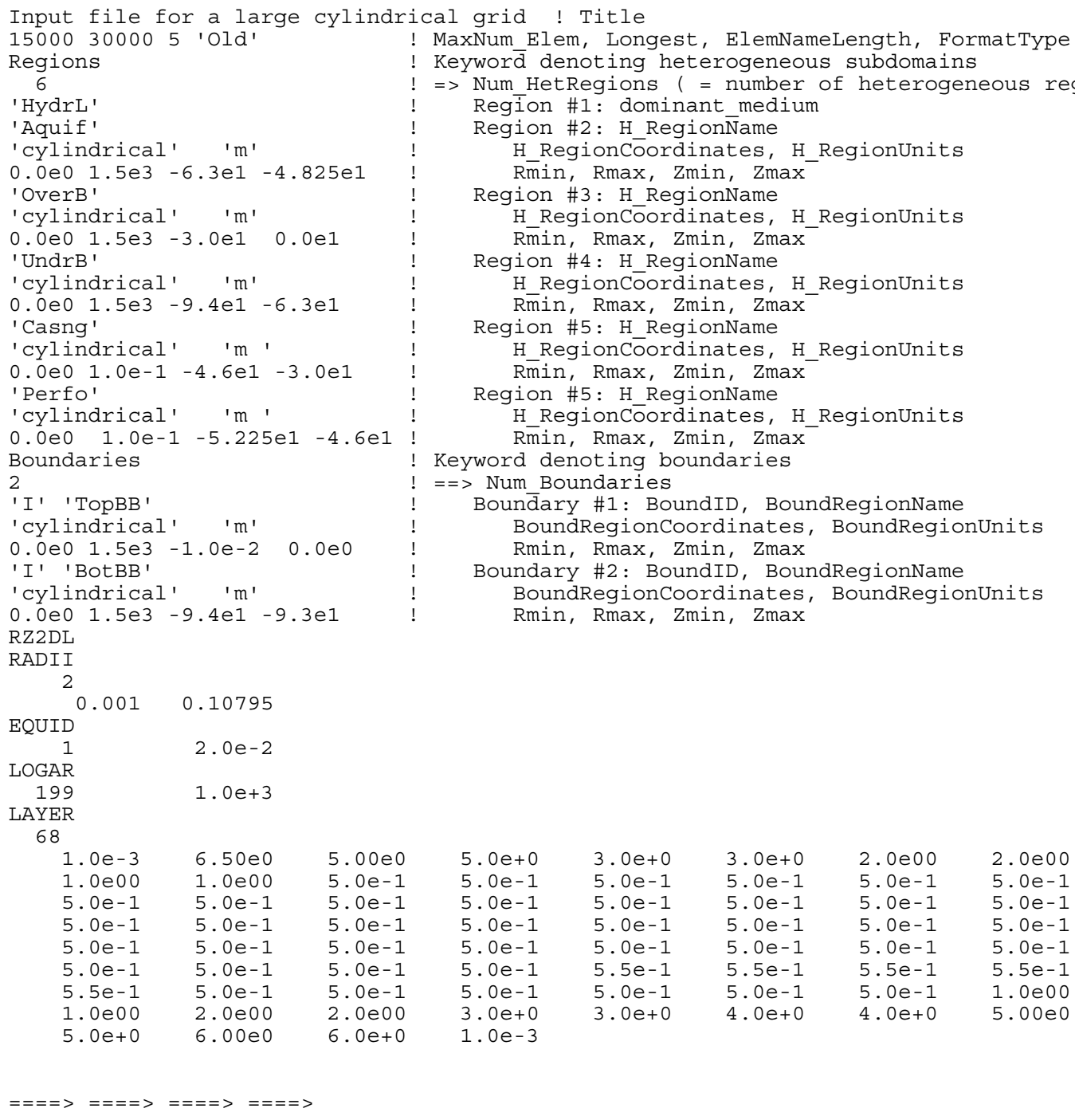

Figure 7.4. An example of a MeshMaker . 995 input file for the creation of a large cylindrical 2D grid with multiple layers, heterogeneous regions and defined boundaries. 


\subsection{Initial Conditions and Boundary Conditions}

In this section the data blocks that allow for domain-specific initial conditions (block INDOM), element-specific initial conditions (INCON), extended capabilities for specifying initial conditions (EXT-INCON), including features for assigning initial conditions to ranges of elements (defined by a variety of methods). Following that the procedure is described for implementing initial conditions and boundary conditions in TOUGH+.

\subsection{Data Block INCON}

This block introduces element-specific initial conditions.

$\underline{\text { Record INCON . } 1 \text { (5-character element names) }}$

Format (A5, 2I5, E15.8, 2x, A3, 36x, 3(E15.8))

ElName5C, NSEQ, NADD, porosity, 
StateIndex, $(\operatorname{perm}(i), \quad i=1,3)$

ElName5C

NSEQ

NADD

porosity

StateIndex

$\operatorname{perm}(i)$
The 5-character name of the element that is being initialized.

The number of additional elements with the same initial conditions.

The increment between the code numbers of two successive elements with identical initial conditions.

The porosity of the element that is being initialized. If porosity is zero or blank, the element porosity will be taken as specified in block ROCKS or MEDIA. This feature is necessary for assignment of element-specific properties for the description of highly or statistically heterogeneous domains.

State identifier (see Section 3.1, Tables 3.1 and 3.2): the initial conditions corresponding to this identifier are applied uniformly over the element.

The intrinsic (absolute) permeabilities of the element that is being initialized along the three directions described by the ConxKi variable (see Section 7.3). If all perm ( $i$ ) , $i=1, \ldots, 3$ are zero or blank, the element permeabilities will be taken as specified in block ROCKS or MEDIA. This feature is necessary for assignment of element-specific properties for the description of highly or statistically heterogeneous domains.

$\underline{\text { Record INCON.2 }}$ specifies primary variables.

Format (6E20.13)

$X(i), i=1$, NumCom +1

The primary variables defining the state of the element specified in record INCON.1. INCON specifications will supersede default conditions specified in PARAM.4 (see Section 10), and domainspecific conditions that may have been specified in data block INDOM. See Section 3.1 (Tables 3.1 and 3.2) for a detailed description of the potential sets of primary variables.

$\underline{\text { Record INCON } .3}$ 
There are three ways to close the INCON data block.

If the simulation is not a continuation run, then a blank line or a record with ' $<<<$ ' typed in the first three columns closes the INCON data block.

In continuation runs from a previous TOUGH [Pruess, 1987] or TOUGH2 [Pruess, 1991; Pruess et al., 1999] simulation, the INCON data block may terminate on a record with ' +++ ' typed in the first three columns, followed by data that describe timing and time stepping information.

In continuation runs from a previous TOUGH+, the INCON data block may terminate on a record with ' $::$ :' typed in the first three columns. This is followed by a data describing (a) the origin of time and the simulated time at the conclusion of the preceding TOUGH+ simulation, (b) the number of timesteps and Newtonian iterations, and (c) accumulated quantities, e.g., total amounts of fluids produced/injected during the previous run (see Figure 8.1). These data are recorded automatically at the end of the SAVE file (see Section 8.4) upon completion of the previous TOUGH+ run using a NAMELIST format. This is a very powerful format that allows maximum clarity and flexibility, accepting free formats, arbitrary ordering of variables, insertions of comments anywhere in the input fields, and providing the option of ignoring any of the NAMELIST parameters by not assigning a value to it. For more information, the reader is directed to a textbook on FORTRAN 95/2003.

$\underline{\text { Record INCON . } 1 \text { (8-character element names) }}$

Format (A8, 7X, E15.8, 2x, A3, 36x, 3(E15.8))

ElName8C, NSEQ, NADD, porosity, StateIndex, $(\operatorname{perm}(i), i=1,3)$

ElName8C

The 8-character name of the element that is being initialized.

All other variables remain as in the case of five-character element names. 


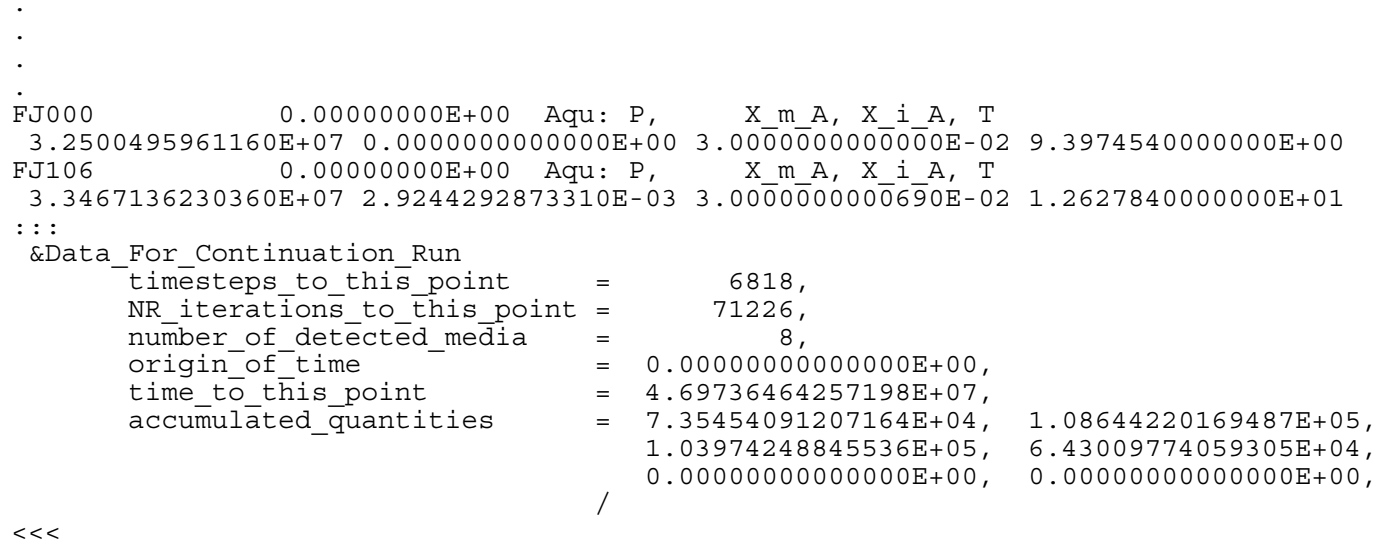

Figure 8.1. An example of the NAMELIST-described termination data printed at the end of the SAVE file from a TOUGH+ simulation. These data can be read as part of the INCON data block, or of the INCON external file. The names of the variables defined in the NAMELIST are selfexplanatory. For reference, this figure lists the conditions in the last two elements (FJ000 and FJ106) of the grid in the TOUGH+ simulation.

\subsection{Data Block INDOM}

This block introduces domain-specific initial conditions. These will supersede default initial conditions specified in PARAM.4 (see Section 10), and can be overwritten by element-specific initial conditions in data block INCON or data block EXT-INCON. The option START is needed to use INDOM conditions.

\section{Record INDOM. 1}

Format (A5, 2X, A3)

Rk_name, StateIndex

$\mathrm{Rk}$ name

StateIndex

Record INDOM. 2
The name of a medium (corresponding to a system subdomain), as specified in data block ROCKS.

The state identifier describing the conditions applying to the Rk_name medium/subdomain. 
Format (6E20.13)

$\mathrm{X}(\mathrm{i}), \quad i=1, \mathrm{NumCom}+1$

$X(i)$ are the primary variables assigned to all grid blocks in the domain specified in record INDOM.1. See Section 3.1 (Tables 3.1 and 3.2) for description of the potential sets of primary variables.

\section{Record INDOM. 3}

A blank record closes the INDOM data block.

Repeat records INDOM. 1 and INDOM. 2 for as many domains as desired. The ordering is arbitrary and need not be the same as in block ROCKS.

\subsection{Data Block EXT - INCON}

This block introduces extended capabilities for specifying initial conditions of subdomains (i.e., groups of element). The user has several options to provide element information (e.g., an element name or number list, location, element sequence, columnar structure, etc.) that defines a subdomain to be initialized with the specified conditions. The first entry is the total number of initialization entities entered within the block. Following that, each of the various input entities may be entered in an arbitrary order.

Record EXT-INCON. 0

This record includes the single integer variable Total_input_num that describes the total number of input entities (representing initial conditions in particular subdomains) that will be entered. This is read using a free format.

\section{$\underline{\text { Record EXT-INCON. } 1}$}

This record includes the single character variable INTYPE that is read using a free format, and which provides a keyword defining the type of data describing the subdomain to be initialized. INTYPE can assume one of the following values: 
'GEOMETRY ', 'LIST ', 'SEQUENCE ', or 'COLUMN ' . Thus, INTYPE determines what kind of variables/data will be read in record in EXT-INCON . 2.

\subsubsection{Data Block GEOMETRY}

The data in this block are read when INTYPE = 'GEOMETRY', and define a set of elements in a subdomain that is bounded within prescribed minimum and maximum coordinates. All elements within this range will be assigned the initial conditions entered in the record EXT-INCON. 3.

$\underline{\text { Record EXT-INCON . } 2.0}$

For a Cartesian grid (coordinate_system = 'Cylindrical', see Section 5), the following real variables are read in EXT-INCON . 2 using a free format:

$$
\text { Xmin, Xmax, Ymin, Ymax, Zmin, Zmax }
$$

These parameters are defined as follows:

Xmin, Xmax

Ymin, Ymax

Zmin, Zmax
Real variables indicating the range of the subdomain to-be-initialized along the $x$-axis of the Cartesian coordinate system.

Real variables indicating the range of the subdomain to-be-initialized along the $y$-axis of the Cartesian coordinate system.

Real variables indicating the range of the subdomain to-be-initialized along the $z$-axis of the Cartesian coordinate system.

For a cylindrical grid (coordinate_system = 'Cartesian', see Section 5), the following real variables are read in EXT-INCON . 2 using a free format:

$$
\text { Rmin, Rmax, Zmin, Zmax }
$$

These parameters are defined as follows: 
Rmin, Rmax

Zmin, Zmax
Real variables indicating the range of the subdomain to-be-initialized along the $r$-axis of the cylindrical coordinate system.

Real variables indicating the range of the subdomain to-be-initialized along the $z$-axis of the cylindrical coordinate system.

\subsubsection{Data Block SEQUENCE}

For INTYPE = 'SEQUENCE'

$\underline{\text { Record EXT-INCON. } 2.0}$

Format (*)

SequICFirstElemNum, SequICLastElemNum, SequICStride

SequICFirstElemNum Integer describing the global number of the first element in the sequence.

SequICLastElemNum Integer describing the global number of the last element in the sequence.

SequICStride Integer describing the stride in the numbering sequence

Thus, a sequence of elements is defined by the beginning and ending element number, as well as by the stride (number of elements to skip between two successive elements in the subdomain defined by the sequence). For example, a sequence with SequICFirstElemNum = 10, SequICLastElemNum = 20 , and SequICStride $=2$ results in the a group (subdomain) of elements with the following global element numbers: 10, 12, 14, 16, 18, 20. If SequICLastElemNum < SequICFirstElemNum or if SequICStride $<0$, an error message is printed and the simulation is aborted.

All elements within this sequence will be assigned the initial conditions entered in the record EXT-INCON. 3. 


\subsubsection{Data Block LIST}

For INTYPE $=$ 'LIST'

$\underline{\text { Record EXT-INCON . } 2.0}$

Format (*)

ListICLength, N_per_row

ListICLength Integer denoting the length of the list (i.e., the total number of element numbers in the list) that is about to be read.

$\mathrm{N}$ _per_row Integer defining the number of entries (= element numbers) per row in the list that that is about to be read. The last row may have fewer than N_per_row entries.

Record EXT-INCON.2.1

Format (*)

ElemNum(i), i=1, ListICLength

NOTE: The number of entries per row of the ElemNum ( $i$ ) input data is N_per_row

The element numbers that are defined through their participation in a list are read in rows (= records), each (except possibly the last one) containing N_per_row entries. The last row/record may have fewer than N_per_row entries. For example, if N_per_row, and N_per_row $=8$, then the element numbers would be listed as follows:

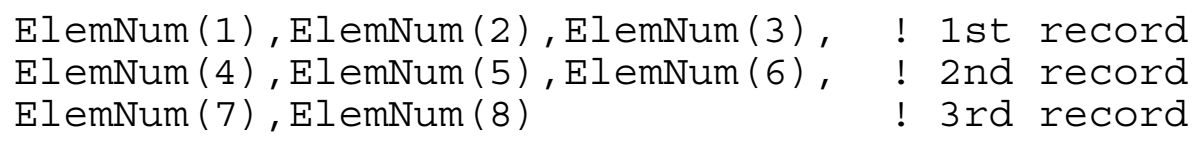

All elements in this list will be assigned the initial conditions entered in the following record (EXT-INCON. 3). 


\subsubsection{Remaining Data Blocks in EXT - INCON}

For INTYPE = 'GEOMETRY', 'SEQUENCE', or 'LIST'

Record EXT-INCON. 3

Format $(*)$

StateIndex, $\mathrm{x} 0(\mathrm{i}), i=1, \mathrm{NumCom}+1$

StateIndex A character variable indicating the state index (see Section 3, Tables 3.1 and 3.2) corresponding to the initial conditions that are to be assigned to the subdomain defined by INTYPE.

Xo (i) A real array that includes the primary variables (corresponding to StateInd) that describe the conditions to which the respective subdomain is initialized. See Section 3.1 for a thorough description of the potential sets of primary variables.

Repeat records EXT-INCON.3, EXT-INCON. 2 and EXT-INCON. 3 for a total of Total_input_num subdomain-based initializations.

\subsubsection{Data Block COLUMN}

This data block is somewhat different from the previous ones, and is very useful in applying initial conditions in problems that involve initialization after achieving gravity equilibration in a single column of the domain, which then serves as the reference column. Thus, when INTYPE = 'COLUMN', the following records and data are read:

\section{$\underline{\text { Record EXT-INCON.2.0 }}$}

This record includes the single integer variable ColmICSize that is read using a free format, and which describes the column length, i.e., the number of elements in the column. 
Record EXT-INCON.2.1

Format (*)

ColmICFirstelemNum, ColmICNumElemInCol, ColmICstride

ColmICFirstElemNum An integer variable indicating the global element number of the first element in the columnar structure to be initialized.

ColmICNumElemInCol An integer variable describing the total number of elements to be initialized using the columnar structure. Thus, the total number of columns to be initialized using the data provided in this block is: ColmICNumElemInCol/ColmICSize

ColmICstride

An integer variable describing the stride in the numbering sequence. This number is the difference between the global numbers of two elements that two successive locations in the same column.

For example, if ColmICSize=10, ColmICFirstElemNum=1, ColmICNumElemInCol $=80$, and ColmICstride $=1$, then initialization using the COLUMN data block will assign the initial conditions (obtained from the 10 elements of the reference column) to columns composed of elements with the following global numbers: Column \#1, elements 1 to 10; Column \#1, elements 11 to $20 ; \ldots$ Column $\# 8$, elements 71 to 80 . In this case, elements $1,11,21, \ldots, 71$ have the same initial conditions (equal to those of the first entry in the reference column). Similarly, elements $2,12,22, \ldots, 72$ have all the same initial conditions (equal to those of the second entry in the reference column).

Conversely, if ColmICstride=1, then elements 1 to 8 have all the same initial conditions (equal to those of the first entry in the reference column), 2 to 16 have all the same initial conditions (equal to those of the second entry in the reference column), etc.

$\underline{\text { Record EXT-INCON } .3 . x, x=1, \ldots, \text { ColmICSize }}$

Format (*)

StateIndex, (Xo (i), i=1, NumCom +1 )

A total of ColmICSize records are read, each providing the state index and primary variables of the elements of the reference column. The variables in these records are as previously defined (see Section 8.3.4). 


\subsection{Implementing Initial Conditions}

Flow systems are initialized by assigning a complete set of primary thermodynamic variables to all grid blocks into which the flow domain is discretized. Various options are available in a hierarchical system, as follows. During the initialization of a TOUGH+ run, all grid blocks are first assigned to default thermodynamic conditions specified in data block PARAM. The defaults can be overwritten for selected reservoir domains by assigning domain-specific conditions in data blocks INDOM or EXT_INCON. These in turn may be superseded by thermodynamic conditions assigned to individual grid blocks in data block INCON. A disk file INCON written to the same specifications as data block INCON may also be used.

The possible sets of primary variables are discussed in Section 3.1 (Tables 3.1 and 3.2), with the actual primary variables depending on the fluid/solid phase composition. During phase change primary variables will be automatically switched from one set to another. In multiphase flow systems, therefore, different grid blocks will in general have different sets of primary variables, and must be initialized accordingly.

For many applications, special initial conditions are needed, such as gravity-capillary equilibrium, or steady state corresponding to certain mass and heat flows. This can be realized by performing a series of TOUGH+ runs, in which thermodynamic conditions obtained in one run, and written to disk file SAVE, are used as initial conditions in a subsequent continuation run. For example, in a hydrate accumulation simulation, a first run may be made to obtain hydrostatic pressure conditions. These may subsequently be used as boundary conditions in a second run segment to simulate undisturbed natural 
state conditions with through-flow of mass and heat. This could be followed by a third run segment with fluid production and injection.

Restarting of a TOUGH+ run is accomplished by renaming the file SAVE generated in a previous run as file INCON for initialization. Usually additional (often minor) adjustments will be made for a restart. For example, different specifications for the number of time steps and desired printout times may be made. Some editing of the MESH file may be needed to make certain grid blocks inactive, so that previously calculated pressures can serve as boundary conditions (see below). In a continuation run, simulation time and time step counters may be continuously incremented, or they may be reset to zero. For example, the latter option will be used when simulating production and injection operations following preparation of a natural initial state, which may correspond to a large simulation time.

As far as the internal workings of the code is concerned, there is no difference between a fresh start of a simulation and a restart. The only feature that makes a simulation a continuation run is that the INCON data were generated by a previous TOUGH2 or TOUGH+ run, rather than having them explicitly provided by the user.

The file SAVE originating from TOUGH2 simulations [Pruess et al., 1991] always ends with a data record with ' +++ ' in the first three columns, followed by one record with restart information (time step and iteration counters, simulation time). The file SAVE originating from a $\mathrm{TOUGH}+$ simulation terminates with continuation data written using the NAMELIST format shown in Figure 8.1. In either case, to reset all counters and continuation data to zero when using SAVE as file INCON for another TOUGH+ run, 
users can simply replace all records below the conditions of the last element with a single blank record.

\subsection{Implementing Boundary Conditions}

Boundary conditions can be of two basic types. Dirichlet conditions prescribe thermodynamic conditions, such as pressure, temperature, etc. on the boundary, while Neumann conditions prescribe fluxes of mass or heat crossing boundary surfaces. A special case of Neumann boundary conditions is no flux, which is the default in the integral finite difference framework when no flow connections are specified across the boundary. More general flux conditions are prescribed by introducing sinks or sources of appropriate strength into the elements adjacent to the boundary.

In TOUGH2 [Pruess et al., 1999], Dirichlet conditions could be implemented by assigning very large volumes (e.g., $V=10^{50} \mathrm{~m}^{3}$, as described by the elem_vol variable in Section 7.2) to grid blocks adjacent to the boundary, so that their thermodynamic conditions do not change at all from fluid or heat exchange with finite-size blocks in the flow domain. In addition, a small value (such as $10^{-9} \mathrm{~m}$ ) should be specified for the nodal distance (ConxD1 or ConxD2, see Section 7.3) of such blocks, so that boundary conditions are in fact maintained in close proximity to the surface where they are desired, and not at some distance from it. It is possible to specify a nodal distance that is outright zero; however, this may interfere with options for the computation of interface mobilities that are intended for modeling fracture-matrix interactions. Therefore, assigning zero nodal distances (ConxD1 or ConxD2) should be used with caution. 
For time-independent Dirichlet boundary conditions, TOUGH2 offered an alternative implementation, which provided savings in computational work along with added user conveniences in running simulation problems. This was accomplished by defining active and inactive elements. By convention, elements encountered in data block ELEME (or in geometry files MESH or MINC) were taken to be active until the first element entry with a zero or negative volume was encountered. The first element with volume elem_vol $\leq$ $0.0 e 0$, and all subsequent elements, were by convention taken to be inactive. The easiest way to declare selected grid blocks as inactive was to use a text editor to move them to the end of the ELEME data block, and then insert a dummy grid block of zero volume in front of them.

TOUGH + maintains these two older options available in TOUGH2, but provides an additional one that is much simpler as it does not require any editing of the element volume or physically moving any portions of the element list. In $\mathrm{TOUGH}+$, elements can be designated as inactive if the parameter element_activity in record ELEME. 1 (see Section 7.2) is set to ' I' (when its conditions are time-invariant) or 'V' (when its conditions and properties vary over time). Then, these elements, as well as all elements designated as inactive by the older TOUGH2 options, are treated as inactive.

For the inactive elements no mass or energy balance equations are set up, their primary thermodynamic variables are not included in the list of unknowns, and their thermodynamic conditions remain unchanged during the course of the simulation. Inactive elements can appear in flow connections and initial condition specifications like all other elements. The computational overhead of inactive elements is small because they do not increase the number of equations to be solved in a flow problem. 


\subsection{Sources and Sinks}

\subsection{Data Block GENER}

This block introduces sinks and/or sources to the system

$\underline{\text { Record GENER. } 1 \text { (for 5-character element names) }}$

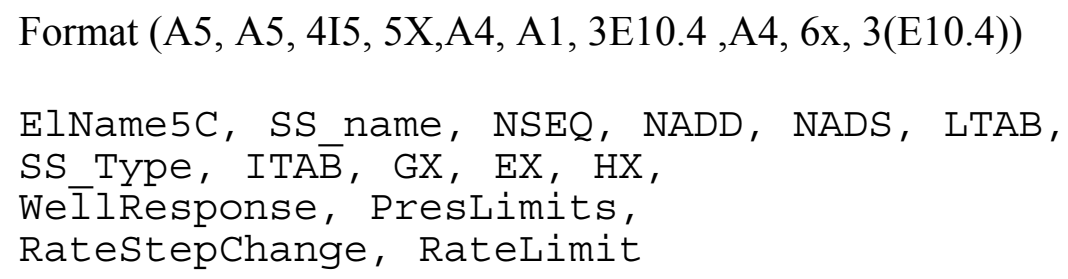

ElName 5C

SS_name

NSEQ
The code name of the 5-character element containing the sink/source.

The name of the sink/source. The first three characters are arbitrary, the last two characters must be numbers.

The number of additional sinks/sources with the same injection/production rate (not applicable for SS_Type $=$ 'DELV' . 
NADD

NADS

LTAB

SS_Type
The increment between the code numbers of two successive elements with identical sink/source.

The increment between the code numbers of two successive sinks/sources.

The number of points in table of generation rate versus time. Set 0 or 1 for constant generation rate. For wells on deliverability, LTAB denotes the number of open layers, to be specified only for the bottommost layer.

The type of source or sink. This variable specifies different options for fluid or heat production and injection. For example, different fluid components may be injected, the nature of which depends on the EOS module being used. Different options for considering wellbore flow effects may also be specified. The following options are available:

$=$ 'HEAT': Introduces a heat sink/source. This option is to be used for injection only.

$={ }^{\prime}$ COM1 1 : Indicates mass component \#1 (usually water). This option is to be used for injection only.

$=$ 'WATE': Indicates water injection.

$=$ ' COM2 ': Indicates mass component \#2. This option is to be used for injection only.

$=' \mathrm{COM} 3 \mathrm{\prime}$ : Indicates mass component \#3. This option is to be used for injection only.

$=$ 'COMn ': Indicates mass component \#n. This option is to be used for injection only.

= 'MASS ': Specified a mass production rate, i.e., the mass rate of all the fluids withdrawn from a system.

= ' DELV': Well on deliverability, i.e., production occurs against specified bottomhole pressure. If well is completed in more than one layer, bottommost layer must be specified first, with number of layers given in 
LTAB. Subsequent layers must be given sequentially for a total number of LTAB layers.

F--- or f--- well on deliverability against specified wellhead pressure. By convention, when the first letter of a type specification is $\mathrm{F}$ or $\mathrm{f}, \mathrm{TOUGH}+$ will perform flowing wellbore pressure corrections using tabular data of flowing bottomhole pressure vs. flow rate and flowing enthalpy. The tabular data used for flowing wellbore correction must be generated by means of a wellbore simulator ahead of a TOUGH+ run according to certain format specifications (see Table 17, section 7.3 in Pruess et al., 1991). The data must be provided by means of a disk file, whose name consists of the four characters of the SS Type specification, and the one character of the following ITAB parameter. For example, to use wellbore pressure data in a disk file called $\mathrm{f} 120 \mathrm{a}$, specify SS_Type as ' $\mathrm{f} 120$ ', and specify ITAB as 'a'. Different wellbore tables, representing e.g. wells with different diameter, feed zone depth, and flowing wellhead pressure, may be used simultaneously in a $\mathrm{TOUGH}+$ simulation. Also, several wells completed in different grid blocks may reference the same wellbore table.

The capability for flowing wellbore pressure correction is presently only available for wells with a single feed zone.

Unless left blank, a table of specific enthalpies will be read (LTAB > 1 only).

GX

The constant generation rate. GX is positive for injection and negative for production. GX describes a mass rate $[\mathrm{kg} / \mathrm{sec}]$ for generation types COMI, COM2, COM3, etc., and MASS; it describes an energy rate [W] for a HEAT sink/source. For wells on deliverability, GX is the productivity index PI $\left[\mathrm{m}^{3}\right]$ - see Equation (9.2).

The fixed specific enthalpy $[\mathrm{J} / \mathrm{kg}]$ of the fluid for mass injection $(\mathrm{GX}>0)$. For wells on deliverability against fixed bottomhole pressure, EX is the 
HG

WellResponse

PresLimits

RatestepChange

RateLimit bottomhole pressure $P_{w b}[\mathrm{~Pa}]$ at the center of the topmost producing layer in which the well is open.

The thickness of layer [m]. This is to be used only in cases of wells on deliverability with specified bottomhole pressure.

A character variable that describes how the source/sink is to be treated if pressure limits (as described by the variable PresLimits) at the corresponding cell are violated. The following options are available:

$=$ 'STOP': The simulation is halted.

= 'ZERO': The source/sink rate GX is reset to zero, and the simulation continues.

= 'ADJU': The source/sink rate GX is adjusted, and the simulation continues. Note that a simulation involving the WellResponse $=$ 'ADJU' option may involve several successive GX adjustments.

A real variable describing the pressure limit $[\mathrm{Pa}]$ that acts as a trigger for Well Response to be enacted. For injection, pressure in the element containing the source has to exceed PresLimits for changes to be implemented. For production, pressure in the element containing the sink has to fall below PresLimits for changes to be implemented.

A real variable that is used only when WellResponse = 'ADJU'. By convention, RatestepChange is always a positive number. It represents the absolute value of the fraction by which GX is to decrease when the PresLimits criterion is violated. For obvious reasons, $0 \leq$ RatestepChange $\leq 1$.

A real variable describing the minimum rate limit, described as the lowest fraction of the original GX to which the rate is allowed to decline. The simulation stops when RateLimit is reached.

$\underline{\text { Record GENER . } 1 \text { (for 8-character element names) }}$ 
Format (A8, A5, 12X, I5, 5X,A4, A1, 3E10.4 ,A4, 6x, 3(E10.4))

ElName8C, SS name, LTAB, SS Type, ITA $\bar{B}$, GX, EX, HX, WelliResponse, Preslimits, Ratestepchange, RateLimit

ElName8C

The code name of the 8-character element containing the sink/source.

All other variables and parameters are as in the case of the 5-character elements.

Record GENER. I. I (Optional, LTAB > 1 only)

Format (4E14.7)

$\mathrm{Fl}(\mathrm{k}), \mathrm{k}=\mathrm{l}, \mathrm{LTAB}$

F1 (k) Generation times in the table of the time variable source/sink data.

Record GENER. 1.2 (Optional, LTAB > 1 only)

Format (4E14.7)

F2 $(k), k=1, \quad$ LTAB

F2 (k) Generation rates in the table of the time variable source/sink data.

Record GENER. 1.3 (Optional, LTAB $>1$ and ITAB non-blank only)

Format (4E14.7)

F3 $(k), k=1, \quad$ LTAB

F3 (k) Specific enthalpy of produced or injected fluid in the table of the time variable source/sink data.

Repeat records GENER.1, GENER.1.1, GENER.1.2, and GENER.1.3 for the desired number of sinks/sources.

Record GENER . 2

A blank record closes the GENER data block. Alternatively, generation information may terminate on a record with ' +++ ' typed in the first three columns, followed by data describing the numbers of the elements where the sources/sinks are located. 


\subsection{Discussion on sinks and sources}

Sinks and sources are introduced through data block GENER in the input file. Several options are available for specifying the production $(q<0)$ or injection $(q>0)$ of fluids and heat. Any of the mass components may be injected in an element at a constant rate, or at time-dependent rates that may be prescribed through user-defined tables. The user has the option of specifying the specific enthalpy of the injected fluid as either a constant or time dependent value. Heat sources/sinks (with no mass injection) may be either constant or time-dependent.

Fluid production from an element may be handled by prescribing a constant or timedependent mass rate. In this case, the phase composition of the produced fluid may be determined by the relative phase mobilities in the source element. Alternatively, the produced phase composition may be specified to be the same as the phase composition in the producing element. In either case, the mass fractions of the components in the produced phases are determined by the corresponding component mass fractions in the producing element. Different options are available for interpolating time-dependent rates from user-supplied tabular data; these may be selected through parameter MOP (12) see Section 10.

\subsubsection{Deliverability Model}


Production wells may operate on deliverability against a prescribed flowing bottomhole pressure $P_{w b}$ with a productivity index PI [Coats, 1977]. With this option, the mass production rate of phase $\beta$ from a grid block with phase pressure $P_{\beta}>P_{w b}$ is

$$
q_{\beta}=\frac{k_{r \beta}}{\mu_{\beta}} \rho_{\beta} \cdot P I \cdot\left(P_{\beta}-P_{w b}\right)
$$

For steady radial flow the productivity index is given by [Coats, 1977; Thomas, 1982]

$$
(P I)_{l}=\frac{2 \pi\left(k \Delta z_{l}\right)_{l}}{\ln \left(\frac{r_{e}}{r_{w}}\right)+s-\frac{1}{2}}
$$

Here, $\Delta \mathrm{z}_{l}$ denotes the layer thickness, $(\mathrm{k} \Delta \mathrm{z})_{l}$ is the permeability-thickness product in layer $l, r_{e}$ is the grid block radius, $r_{w}$ is the well radius, and $s$ is the skin factor. If the well produces from a grid block that does not have cylindrical shape, an approximate $P I$ can be computed by using an effective radius

$$
r_{e}=\sqrt{\frac{A}{\pi}}
$$

where $A$ is the grid block area; e.g., $A=\Delta x \Delta y$ for an areal Cartesian grid. More accurate expressions for specific well patterns and grid block shapes have been given in the literature [e.g., Peaceman, 1977, 1982; Coats and Ramesh, 1982].

The rate of production for mass component $\kappa$ is

$$
\hat{q}^{\kappa}=\sum_{\beta} X_{\beta}^{\kappa} q_{\beta}
$$

For wells which are screened in more than one layer (element), the flowing wellbore pressure $P_{w b}$ can be corrected to approximately account for gravity effects according to the depth-dependent flowing density in the wellbore. Assume that the open interval extends from layer $l=1$ at the bottom to $l=\mathrm{L}$ at the top. The flowing wellbore pressure 
in layer $l, P_{w b, l}$, is obtained from the wellbore pressure in layer $l+1$ immediately above it by means of the following recursion formula

$$
P_{w b, l}=P_{w b, l+1}+\frac{g}{2}\left(\rho_{l}^{f} \Delta z_{l}+\rho_{l+1}^{f} \Delta z_{l+1}\right)
$$

Here, $g$ is acceleration of gravity, and $\rho_{l}^{f}$ is the flowing density in the tubing opposite layer $l$. Flowing densities are computed using a procedure given by Coats [private communication with K. Pruess, 1982]. If wellbore pressure were zero, we would obtain the following volumetric production rate of phase $\beta$ from layer $l$.

$$
r_{l, \beta}=\left(\frac{k_{r \beta}}{\mu_{\beta}}\right)_{l}(P I)_{l} P_{l, \beta}
$$

The total volumetric flow rate of phase $\beta$ opposite layer $l$ is, for zero wellbore pressure

$$
r_{l, \beta}^{T}=\sum_{m=1}^{l} r_{m, \beta}
$$

From this we obtain an approximate expression for flowing density opposite layer $l$, which can be used in Equation (9.5).

$$
\rho_{l}^{f}=\sum_{\beta} \rho_{l, \beta} r_{l, \beta}^{T} / \sum_{\beta} r_{l, \beta}^{T}
$$

During fluid production or injection, the rate of heat removal/injection is determined by

$$
\hat{q}^{h}=\sum_{\beta} q_{\beta} h_{\beta}
$$

where $h_{\beta}$ is the specific enthalpy of phase $\beta$. 


\subsubsection{Coupled Wellbore Flow}

Geothermal production wells typically operate at (nearly) constant wellhead pressures. As flow rate and flowing enthalpy change with time, wellbore pressure gradients and flowing bottomhole pressures will also change. From a conceptual point of view, the most straightforward way to describe production from geothermal wells is to set up and solve equations for flow in the reservoir and flow in the wellbore in a fully coupled manner. This approach was taken by Hadgu et al. [1995], who coupled the reservoir simulator TOUGH [Pruess, 1987] with the wellbore simulator WFSA [Hadgu and Freeston, 1990].

As discussed by Murray and Gunn [1993], an alternative approach may be preferable in which the wellbore and reservoir simulations are performed separately. This can be accomplished by running a wellbore flow simulator prior to the reservoir simulation for a range of flow rates $\mathrm{q}$ and flowing enthalpies $\mathrm{h}$, in order to generate a table of flowing bottomhole pressures $P_{w b}$.

$$
P_{w b}=P_{w b}\left(q, h ; P_{w h}, z, r_{w}\right)
$$

In addition to the functional dependence on $q$ and $h$, flowing bottomhole pressure is dependent on a number of well parameters. These include wellhead pressure $P_{w h}$, feed zone depth $z$, wellbore radius $r_{w}$, friction factors, and possibly others. By interpolating on these tabular data, Equation (9.10) can be directly inserted into the well source term, Equation (9.1). Reservoir flow equations that include a quasi-steady approximation to wellbore flow can then be solved with little added computational expense compared to the case where no wellbore flow effects are considered. Advantages of representing wellbore flow effects through tabular data include increased robustness and 
computational efficiency. It also makes it possible to use different wellbore simulators and two-phase flow correlations without any programming changes in the reservoir simulation code.

We have incorporated a tabular interpolation scheme for dynamic changes of flowing bottomhole pressure into $\mathrm{TOUGH}+$. Flowing enthalpy at the well feed is known from phase mobilities and enthalpies calculated by the reservoir simulator. The unknown well flow rate and flowing bottomhole pressure are obtained by Newton-Raphson iteration on

$$
R(q) \equiv q-\left(\sum \frac{k_{r \beta}}{\mu_{\beta}} \rho_{\beta}\right) \cdot P I \cdot\left[P-P_{w b}(q, h)\right]=0
$$

The iterative solution of Equation (9.11) was embedded in the outer (Newtonian) iteration performed by $\mathrm{TOUGH}+$ on the coupled mass and heat balance equations. Additional computational work in comparison to conventional simulations with constant downhole pressure is insignificant.

Coupled wellbore flow is invoked in a TOUGH+ simulation by specifying a GENERtype starting with the letters ' $F$ ' or ' $f$ '. For example, ' $f 725 d$ ' is a valid GENER-type that will associate the host element with a production well. If this is specified, then the user must supply a disk file named $\mathrm{f} 725 \mathrm{~d}$ with appropriately formatted data that relate wellhead to flowing bottomhole pressures. Several wells may use the same wellbore pressure table, and multiple data sets for flowing wellbore pressures may be used, e.g., for wells with different diameter, feed zone depth, and wellhead pressure. The well table(s) must be generated by wellbore simulation prior to a TOUGH+ run as ASCII data of 80 characters per record, as described in the example given in Table 17 of Pruess et al. [1991]. 
The first record is an arbitrary title. The second record holds the number of flow rate and flowing enthalpy data (table points), NG and NH, respectively, in Format 2I5; in Table 17 of Pruess et al. [1991] we have NG=11, NH=9. This is followed by NG flow rate data in format $8 \mathrm{E} 10.4$, and $\mathrm{NH}$ enthalpy data also in format $8 \mathrm{E} 10.4$. After this come NG sets of $\mathrm{NH}$ flowing bottomhole pressure data in format 8E10.4. The data in Table 17 of Pruess et al. [1991] were generated with the HOLA wellbore simulator [Aunzo et al., 1991] for a $0.2 \mathrm{~m}(\approx 8 \mathrm{inch})$ inside diameter well of $1,000 \mathrm{~m}$ feed zone depth with 7 bars wellhead pressure. Formation temperature for the conductive heat loss calculation in HOLA was assumed to increase linearly from $25{ }^{\circ} \mathrm{C}$ at the land surface to $275.5^{\circ} \mathrm{C}$ at $750 \mathrm{~m}$ depth. Flow rates cover the range from 0.5 to $90.5 \mathrm{~kg} / \mathrm{s}$, and flowing enthalpies cover the range from 1,000 to $1,400 \mathrm{~kJ} / \mathrm{kg}$. A data record with very large bottomhole pressures of 55.55 MPa was added by hand for a very large hypothetical rate of $1,000 \mathrm{~kg} / \mathrm{s}$. This was done to avoid rates going out of table range during the Newton-Raphson iteration in a TOUGH2 flow simulation, thus resulting in more robust time stepping behavior (see Section 9.5 and Figure 10 in Pruess et al. [1991]).

The coupled wellbore flow capability as coded in TOUGH+ is limited to wells with a single feed zone and can only handle wellbore pressure effects from changing flow rates and enthalpies. Effects from changing fluid composition, as e.g. variable noncondensible gas content, are not modeled at present. 


\section{Computational Parameters}

In this section the data blocks (PARAM and SOLVR) that specify computational parameters are described and followed by a discussion on linear equation solvers.

\subsection{Data Block PARAM}

This block describes an assortment of computation parameters.

\section{$\underline{\text { Record PARAM . } 1}$}

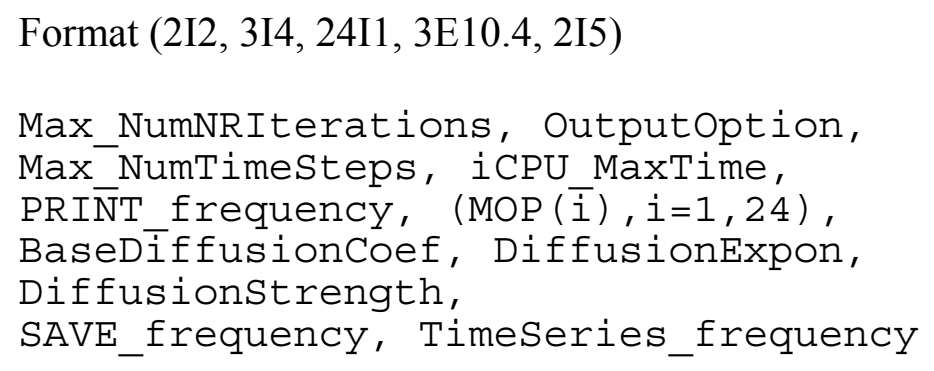

Max_NumNRIterations Integer specifying the maximum number of Newtonian iterations per time step (default is 8) 
Outputoption

Max_NumTimesteps

iCPU_MaxTime

PRINT_frequency

$\operatorname{MOP}(i), \quad i=1,24$

$\operatorname{MOP}(1)$

$\operatorname{MOP}(2)$ to $\operatorname{MOP}(6)$
Integer defining an option that controls the amount of printout in the standard TOUGH+ output (the default is 1). The following options are available:

$=0,1:$ Print a selection of the most important variables.

$=2:$ In addition, print the mass and heat fluxes and the flow velocities.

$=3:$ In addition, print the primary variables and their changes.

If the above values for Outputoption are increased by 10 , printout will occur after each Newton-Raphson iteration (not just after convergence).

An integer defining the maximum number of time steps allowed during the simulation.

NOTE: If Max_NumTimesteps < 0, then the maximum number of timesteps becomes $1000 *$ ABS (Max_NumTimesteps)

An integer describing the maximum duration, in CPU seconds, of the simulation (default is infinite).

A integer describing the printout frequency. This, printout will occur for every multiple of PRINT_frequency steps (the default is 1).

NOTE: If PRINT_frequency < 0 , then the printout frequency becomes $1000 *$ ABS (PRINT_frequency)

An integer array that allows choice of various computational options. These are described in detail below, and are documented in the printed standard output from a TOUGH+ run.

If $\operatorname{MOP}(1) \neq 0$, a short printout for non-convergent iterations will be generated at the end of each Newton-Raphson iteration.

If $\neq 0$, these options generate additional printout in various subroutines at the end of each Newton- 
MOP ( 2)

$\operatorname{MOP}(3)$

$\operatorname{MOP}(4)$

$\operatorname{MOP}(5)$

$\operatorname{MOP}(6)$

$\operatorname{MOP}(7)$

$\operatorname{MOP}(8)$ raphson iteration. This feature is not needed in normal applications, but can be convenient in the development of new capabilities as they can be used to identify coding errors. The amount of printout increases with the value of MOP ( $i$ ). The user is encouraged to consult the source code listings for details. Below we list the subroutines corresponding to MOP (2) to MOP (6) :

Simulation_Cycle (executive subroutine that advances time and controls the flow of data during the simulation)

JACOBIAN_SetUp (routine computing the flow and accumulation terms in the mass and energy balance equations).

SourceSink_Equation_Terms (subroutine determining the contribution of sinks/sources to the mass and energy balance equations).

Equation of state (routine describing the equation of state of the system under investigation and computing all thermophysical properties).

Solve Jacobian Matrix Equation (routine that solves the linear equations of the Jacobian matrix).

If $\operatorname{MOP}(7) \neq 0$, a printout of the input data is provided in the standard output file.

It determines how relative permeability and capillary pressure are estimated in the presence of solid phases (see discussion of Section 2.12). The following options are available:

$=0:$ Based on the OPM model; capillary pressure scaling based on EPM \#1.

= 1: Based on EPM \#1 model; capillary pressure scaling based on EPM \#1.

= 2: Based on EPM \#2 model; capillary pressure scaling based on EPM \#2. 
= 3: Based on EPM \#1 model, no capillary pressure scaling.

= 4: Based on EPM \#2 model, no capillary pressure scaling.

= 9: Based on OPM model, no capillary pressure scaling.

$\operatorname{MOP}(9)$

It determines the composition of produced fluid with the MASS option - see discussion on the data block GENER in Section 9. The relative amounts of phases are determined as follows:

$=0:$ according to relative mobilities in the source element.

$=1:$ the produced source fluid has the same phase composition as the producing element.

$\operatorname{MOP}(10)$

It controls the selection of the interpolation formula for the composite heat conductivity as a function of the various phase saturations. The following options are available

$$
\begin{array}{ll}
=0: & k_{\Theta}=k_{d r y}+\left(\sqrt{S_{A}}+\sqrt{S_{H}}\right)\left(k_{w e t}-k_{d r y}\right)+\phi S_{I} \lambda_{I} \\
=1: & k_{\Theta}=k_{d r y}+\left(S_{A}+S_{H}\right)\left(k_{w e t}-k_{d r y}\right)+\phi S_{I} \lambda_{I} \\
=2: & k_{\Theta}=k_{d r y}+\phi\left(S_{A} \lambda_{A}+S_{H} \lambda_{H}+S_{I} \lambda_{I}\right)
\end{array}
$$

(Based on the linear model of Bejan [1984], gas contribution ignored)

$$
\begin{aligned}
=3: & k_{\Theta}=k_{d r y}+\phi\left(S_{A} \lambda_{A}+S_{H} \lambda_{H}+S_{I} \lambda_{I}+S_{G} \lambda_{G}\right) \\
& \text { (Based on the linear model of Bejan [1984], } \\
& \text { including gas contribution) }
\end{aligned}
$$

Options MOP $(10)=0$ and $\operatorname{MOP}(10)=1$ are based on extensions of an earlier model of Somerton et al. [2003; 2004] based on the analysis of Moridis et al. [2005] of the thermal properties of hydrates from laboratory studies [Kneafsey et al., 2005]. It is not known under what conditions (if any) the linear model of Bejan [1984] (invoked for MOP ( 10$)=$ 2 and $\operatorname{MOP}(10)=3$ ) is applicable, but it is 
included for completeness. The option $\operatorname{MOP}(10)=3$ is discouraged because of (a) doubts about the validity of the Bejan [1984] linear model, (b) the very demanding computations for the estimation of the gas thermal conductivity from the real gas property package in $\mathbf{T}+\mathbf{H}$ is demanding, and (c) the small overall contribution to the composite thermal conductivity.

MOP (11)

It provides alternative options for the evaluation of mobility and permeability at interfaces. These are:

$=0:$ The mobilities are upstream weighted according to the W_upstream factor (see discussion in PARAM. 3), and the permeability is upstream-weighted.

$=1:$ The mobilities are averaged between adjacent elements, and the permeability is upstream-weighted.

$=2:$ The mobilities are upstream weighted, and the permeability is harmonic-weighted.

$=3:$ The mobilities are averaged between adjacent elements, and the permeability is harmonic-weighted.

$=4:$ The mobility and permeability are both harmonic weighted.

For multiphase flow simulations in which the upstream element is not known a priori, MOP (11) $=0$ and $\mathrm{MOP}(11)=0$ are the recommended options. The user is strongly cautioned against using other options.

MOP (12)

It determines the interpolation procedure of the tabular data (involving times, flow rates and enthalpies, see Section 8) in time-dependent sources and sinks. The following options are available:

$=0:$ triple linear interpolation; tabular data are used to obtain interpolated rates and enthalpies for the beginning and end of the 
time step; the average of these values is then used.

$=1:$ step function option; rates and enthalpies are taken as averages of the table values corresponding to the beginning and end of the time step.

$=2:$ rigorous step rate capability for time dependent generation data.

A set of times $t_{i}$ and generation rates $q_{i}$ provided in data block GENER is interpreted to mean that sink/source rates are piecewise constant and change in discontinuous fashion at table points.

Specifically, generation is assumed to occur at constant rate $q_{i}$ during the time interval $\left[t_{i}, t_{i+1}\right)$, and changes to $q_{i+1}$ at $t_{i+1}$. The actual rate used during a time step that ends at time $t$, with $t_{i} \leq t \leq$ $t_{i+1}$, is automatically adjusted in such a way that total cumulative exchanged mass at time $t$

$Q(t)=\int_{0}^{t} q d t=\sum_{j=1}^{i-1} q_{j}\left(t_{j+1}-t_{j}\right)+q_{i}\left(t-t_{i}\right)$

is rigorously conserved. If tabular data for enthalpies are also provided, an analogous adjustment is made to fluid enthalpy to preserve $\int q_{h} d t$.

$\operatorname{MOP}(13)$

Option used in processes involving mechanical dispersion.

MOP ( 14)

It specifies the handling of gas solubility in liquid phases according to one of the following options:

$=0:$ The gas solubility is computed using Henry's constants ( $T$-independent).

$=1:$ The gas solubility is computed using appropriate equations of Henry's dissolution parameters ( $T$-dependent).

$>$ 1: The gas solubility is computed using fugacities ( $T$ - and $P$-dependent) 
MOP ( 15)

For low $P$ and $T$ variations, it is possible to use the MOP (14) $=0$ option, which leads to the use of a $P$ and $T$-invariant Henry's constants for the estimation of solubility. Because of the pressure and temperature regime in hydrate simulations, this option is unavailable in $\mathbf{T}+\mathbf{H}$. When $\operatorname{MOP}(14)=1$, solubility is computed from fast parametric equations that describe the effect of temperature. For MOP (14) $>1$, gas solubility is computed from fugacities and activity coefficients. While this approach is accurate over the entire region of pressure and temperature regimes handled by $\mathbf{T}+\mathbf{H}$, it is computationally intensive. In the case of the relatively insoluble $\mathrm{CH}_{4}$, the incremental improvement over the predictions when $\operatorname{MOP}(14)=1$ is marginal, and cannot justify the significantly longer execution times. Thus, this option is deactivated in the current version.

A flag indicating whether conductive heat exchange with impermeable confining layers (see Section 7.4) is to be considered.

$=0:$ The heat exchange is not considered.

$=1:$ The heat exchange is activated (for grid blocks that have a non-zero heat transfer area; see data block ELEME in Section 7.2).

MOP ( 16 )

It provides automatic time step control. Time step size will be doubled if convergence occurs within ITER $\leq$ MOP ( 16 ) Newton-Raphson iterations.

It is recommended to set MOP (16) in the range of 2 - 5. The default value is 4 .

MOP ( 17 )

It specifies the handling of binary gas diffusivities according to one of the following options:

$=0:$ The binary gas diffusivity is computed from the method of Fuller et al. [1969].

= 7: The basic estimate of the binary gas diffusivity is computed from the method of Fuller et al. [1969], and is then adjusted for 
high pressures using the method of Riazi and Whitson [1993].

MOP (18)

MOP (19)
A flag determining the method for estimating interface density.

$=0:$ Perform upstream weighting for interface density.

$>0$ : Use the average interface density between the two grid blocks. However, when one of the two phase saturations is zero, upstream weighting is to be performed.

This is the parameter that controls the simulation output. When MOP $(19)<8$, a standard ASCII file output (as described in general terms by Pruess et al. [1999]) is produced. Depending on the value of the OutputOption parameter in block PARAM . 1, this output can includes pressure, temperature and saturation distribution of the various phases, concentrations, thermophysical properties, and primary and secondary variables.

When MOP $(19)=8$, two additional files containing the most important properties are also printed in a format that conforms to the requirements of the TecPlot package [XXXX,YYYY], and is suitable for most other plotting and graphing packages. The names of these two files are Plot_Data_Coord (containing the Cartesian coordinates of the grid elements) Plot_Data_Elem (which stores the element-specific properties and parameters for plotting and graphing). Depending on the value of the OutputOption parameter, it also possible to obtain the third file Plot_Data_Conx that stores connection-specific properties and parameters for plotting and graphing. For MOP $(19)=9$, the plotting files and a truncated standard output file are produced (listing only mass balances at the prescribed printout times).

MOP (20)
Flag determining whether the validity of the initial conditions is to be checked. The options are: 
$<$ 9: The initial conditions are checked to ensure physically meaningful and noncontradictory state indices and the corresponding primary variable values (default).

$=9:$ No checking of initial conditions is performed.

This option may be useful in continuation runs involving large grids, in which case checking of the initial conditions (as provided by the SAVE file) is both time consuming (in terms of computation time) and generally unnecessary. In general, the user is discouraged from bypassing the checking process.

MOP (21)

A computational parameter to selects the linear equation solver (see Section 10.2) from among the following options:

$=0:$ defaults to $\operatorname{MOP}(21)=3$.

$=1:$ LUBAND, banded direct solver using LU decomposition.

$=2:$ DSLUBC, bi-conjugate gradient solver with preconditioner.

$=3:$ DSLUCS (default), Lanczos-type preconditioned bi-conjugate gradient solver with preconditioner.

$=4:$ DSLUGM, generalized minimum residual preconditioned conjugate gradient solver with preconditioner.

$=5:$ DLUSTB, stabilized bi-conjugate gradient solver with preconditioner.

All conjugate gradient solvers use incomplete LUfactorization as a default preconditioner. Other preconditioners may be chosen by means of the data block SOLVR (see Section 10.2).

MOP (22)

A flag indicating whether an additional output file will be created to list the location and magnitude of the largest pressure in the domain. 
$=9: \mathrm{A}$ time series of the maximum pressure in the domain and its location is printed in the external file MaxP_Time_Series.

$<$ 9: No $\max \{P\}$ output is provided (default).

MOP (23)

MOP (24)

BaseDiffusionCoef

DiffusionExpon

Diffusionstrength

SAVE_frequency
Used by some equations of state. Information on its usage is provided in the User's Manual of the corresponding equations of state.

It determines handling of multiphase diffusive fluxes at interfaces according to one of the following options:

$=0:$ Harmonic weighting of fully coupled effective multiphase diffusivity.

$=1:$ Separate harmonic weighting of the gas and liquid phase diffusivities.

The base gas diffusion coefficient $\left[\mathrm{m}^{2}\right]$

Parameter (exponent) describing the temperature dependence of gas phase diffusion coefficient - see Equation (6.4).

Parameter (optional) describing the effective strength of enhanced vapor diffusion; if set to a non-zero value, it will replace the parameter group $\phi \tau_{0} \tau_{\beta}$ for vapor diffusion - see Equation (2.46) and Section 6.4.1.

Frequency of writing and saving the SAVE file. This feature avoids data loss if the simulation is interrupted. A value between 100 and 500 is recommended. When SAVE_frequency $=0$, the SAVE file is written and stored only once at the conclusion of the simulation.

TimeSeries_frequencyFrequency of writing and saving the data in the time-series output files (files SS_Time_Series, Elem_Time_Series, Conx_Time_Series, and the various RegionXX_Time_Series files). This feature avoids the creation of very large time series files in long simulations that involve 
many thousands of time steps. Note that massbalance related calculations related to some of the parameters included in the time series output files are conducted at the conclusion of each time step, but the results are printed only when specified by this parameter. The default value of Timeseries_frequency $=1$.

\section{Record PARAM . 2}

Format (4E10.4, A5, 5X, 3E10.4)

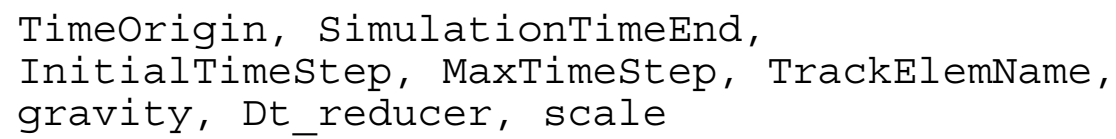

Timeorigin

SimulationTimeEnd

InitialTimestep

MaxTimestep

TrackElemName

gravity

Dt_reducer
A real variable indicating the origin of time (starting time) in the simulation [sec]. The default is Timeorigin $=0.0 e 0$.

A real variable indicating the time [sec] at which simulation should stop. The default is infinite.

A real variable specifying the initial time step size [sec]. If InitialTimestep $<0$, then the program proceeds to read NumDts $=$ INT (ABS (InitialTimestep)) records with time step information.

A real variable defining the upper limit for time step size [sec]. The default is infinite.

A character variable providing the name of an element, the behavior of which is to be tracked over time by printing a short printout of the evolution of its key conditions and properties after each time step.

A real variable specifying the magnitude $\left[\mathrm{m} / \mathrm{sec}^{2}\right]$ of the gravitational acceleration vector. Blank or zero gives "no gravity" calculation.

A real variable defining the factor by which time step is reduced in case of convergence failure or other problems. The default value is 4 . 
scale

The scale factor (a real variable) by which the size of the mesh is adjusted (default $=1.0)$.

Record PARAM.2.1,2.2, etc.

Format (8E10.4)

(UserTimeStep (i), $i=1$, NumDts)

UserTimeStep ( $i$ ) is the length [sec] of time step $i$.

This set of records is optional, and is read only when InitialTimestep $<0$, in which case the integer NumDts $=\operatorname{INT}($ ABS (InitialTimestep)). Up to 13 records can be read, each containing 8 time step data. If

Max_NumTimesteps $>$ NumDts, the simulation will continue with time steps equal to the last non-zero UserTimeStep ( $i$ ) encountered. When automatic time step control is chosen (MOP $(16)>0$ ), time steps following the last UserTimeStep ( $i$ ) input will increase according to the convergence rate of the Newton-Raphson iteration. Automatic time step reduction will occur if the maximum number of Newton-Raphson iterations is exceeded (parameter Max_NumNRIterations, record PARAM.1)

Record PARAM .3

Format (7E10.4, 2X, A3, 3E10.4)

rel convergence crit, abs convergence crit, U_p, W_upstream, W_NRIterātion, dêrivative incremeñt, $W$ implicitness, DefaultstateIndex, P_overshoot, T_overshoot, S_overshoot

rel_convergence_crit Convergence criterion for relative error (real variable, parameter $\varepsilon_{1}$, see Equation (3.8), default = $\left.10^{-5}\right)$.

abs_convergence_critConvergence criterion for absolute error (real variable, parameter $\varepsilon_{2}$, see discussion of Equation (3.9), default =1).

$\mathrm{U} \_\mathrm{p}$

W_upstream
Not used in $\mathrm{TOUGH}+$, maintained only to ensure compatibility with older TOUGH2 input files.

The upstream weighting factor (real variable) for computing mobilities and enthalpies at interfaces. 
The default $\mathrm{W}$ _upstream $=1.0$ is strongly recommended for multi-phase flows.

$(0 \leq$ W_upstream $\leq 1)$.

W_NRIteration

A weighting factor (real variable) determining the level of updating of the solutions based on the results of the Newton/Raphson iteration. The default $W$ NRIteration $=1.0$ is recommended. $(0<$ W_NRIteration $\leq 1)$.

derivative_increment The increment factor (a real variable) for numerically computing derivatives. The default value is derivative_increment $=10^{-m / 2}$, where $m$, evaluated internally, is the number of significant digits of the floating point processor used. For 64-bit arithmetic, derivative_increment $\approx 10^{-8}$.

W_implicitness

DefaultstateIndex

P_overshoot
A weighting factor (a real variable) describing the level of implicitness in the solutions. The default $\mathrm{W}$ implicitness $=1.0$ is recommended. $(0<$ W_implicitness $\leq 1)$.

The default state identifier (a character variable) of the general initial conditions that apply uniformly over the entire if not amended by the data blocks related to the description of initial conditions in subdomains (see Section 8).

A real variable specifying the level of overshoot (defined as a fraction) allowed in the computation of pressure if $P$ is used as a criterion for triggering phase and state changes. When P_overshoot $=$ 0.0 e 0 (default), the $P$-triggered phase and state changes are at their most accurate and sensitive (hair-trigger).

This variable is introduced to alleviate potential problems caused by narrow oscillations about phase equilibrium lines that are possible under certain conditions. In most cases, a value of $10^{-6} \leq$ P_overshoot $\leq 10^{-4}$ is sufficient if a hair trigger causes problems. If $\mathrm{P}_{-}$overshoot $<0$ or P_overshoot $\geq 5.0 \times 10^{-2}$, it is rest internally to its default value $(=0)$. 
T_overshoot

S_overshoot
A real variable specifying the level of overshoot (defined as a fraction) allowed in the computation of temperature if $T$ is used as a criterion for triggering phase and state changes. The definitions, defaults, limits and application are entirely analogous to those of P_overshoot.

A real variable specifying the level of overshoot (defined as a fraction) allowed in the computation of saturations if thearese used criteria for triggering phase and state changes. The definitions, defaults, limits and application are entirely analogous to those of P_overshoot.

\section{Record PARAM. 4}

This record introduces a set of primary variables that are used as default initial conditions for all grid blocks that are not assigned by means of data blocks INDOM, INCON or EXT-INCON. Option START is necessary to use default INCON. The format and date read here are:

Format (6E20.13)

$$
\text { default_initial_cond(i), } i=1, \mathrm{NumCom}+1
$$

As is self evident, the real variables default_initial_cond(i) describe the initial conditions of the state defined by StateIndex, as defined by the corresponding primary variables. When more than six primary variables are needed, more than one line (record) must be provided. See Section 3.1 for description of potential sets of state indices and primary variables. 


\subsection{Modification of Computational Parameters During the Course of a TOUGH+ Simulation}

It is possible to modify the computational parameters described in Section 10.1 in the course of a TOUGH+ simulation without having to interrupt the execution. This feature is particularly useful in simulations involving large grids and a large number of timesteps (Max_NumTimeSteps) when the user observe solution convergence and time advancement that can be improved by varying some of these computational parameters.

The process is controlled by the parameter SAVE_frequency (see Section 10.1, record PARAM. 1) that determines the frequency of updating the SAVE file. At the time of updating the SAVE file, TOUGH+ also interrogates the directory of execution for the presence of a file called Parameter_Update_File. If no such file exists, there is no updating of the computational parameters.

If a file Parameter_Update_File exists, then it is opened and the following data are read:

\section{Record UPDATE . 1}

This record includes the single character variable UpdateHeader that is read using a free format. The TOUGH+ computational parameters are updated only when UpdateHeader = 'Update_Simulation_Parameters'. Otherwise, the Parameter_Update_File is closed and the simulation continues without any parameter updating.

\section{Record UPDATE . 2}

This record includes a set of real variables (computational parameters) that are to be updated. The data in this record are read using a NAMELIST format, and may occupy one or more lines (a choice left to the user). As already discussed (Section 8.1), NAMELIST-based formats are a feature of FORTRAN 95/2003 and provide unique power and flexibility, allowing (a) assignment of updated values to any subset of the parameters included in the NAMELIST definition, (b) arbitrary order, (c) free formats of individual parameter values, (d) inclusion of 
comments, etc. Future versions of TOUGH+ will involve NAMELIST-based formats to read most input data.

The namelist in this record is named Real_Parameters_To_Update, and includes the following real parameters:

(1) SimulationTimeEnd

(2) MaxTimestep

(3) rel_convergence_crit

(4) abs_convergence_crit

(5) P_overshoot

(6) T overshoot

(7) S_overshoot

The corresponding TOUGH+ computational parameters will be updated if values are provided for any of these computational parameters. The structure of the Real_Parameters_To_Update namelist (and its use as an input format) is best illustrated in the example of Figure 10.1.

\section{Record UPDATE. 3}

This record includes a set of integer computational parameters that are to be updated. The data in this record are read using a NAMELIST format. This namelist is named Integer_Parameters_To_Update, and includes the following integer parameters:

(1) Max NumTimesteps

(2) Max NumNRIterations

(3) $\mathrm{MOP}_{-}^{-16}$

(4) SAVE frequency

(5) Timeseries_frequency

The corresponding TOUGH+ integer parameters will be updated if values are provided for any of these computational parameters. It is possible to stop a simulation by providing a Max_NumTimesteps value that is smaller than its current value in the code. Then, the simulation will be halted upon reading the smaller Max NumTimesteps value while at the same time preserving the data in the SAVE file, which would be lost if the execution is interrupted.

The structure of the Integer_Parameters_To_Update namelist (and its use as an input format) is best illustrated in the example of Figure 10.1.

Upon reading the contents of the Parameter_Update_File and updating the computational parameters, $\mathrm{TOUGH}+$ (a) prints a prominent message in the standard output file that provides all the new parameter values, and (b) replaces the UpdateHeader = 'Update_Simulation_Parameters' value with 
the value UpdateHeader $={ }^{\prime}==>$ Completed Update \# $\mathrm{n}$ ', where $\mathrm{n}$ is the number of the update. Because UpdateHeader has no longer the value that will cause TOUGH+ to read the subsequent data, this substitution prevents multiple readings of the same Parameter_Update_File while keeping track of the number of updates and preserving the evolution of the updated parameters in the input file. Note that several updates are possible in the course of a long simulation. To accomplish this, the data for the next update are simply added to the top of the Parameter_Update_File file without erasing the updating history up to this point. The process is clearly illustrated in the example of Figure 10.2.

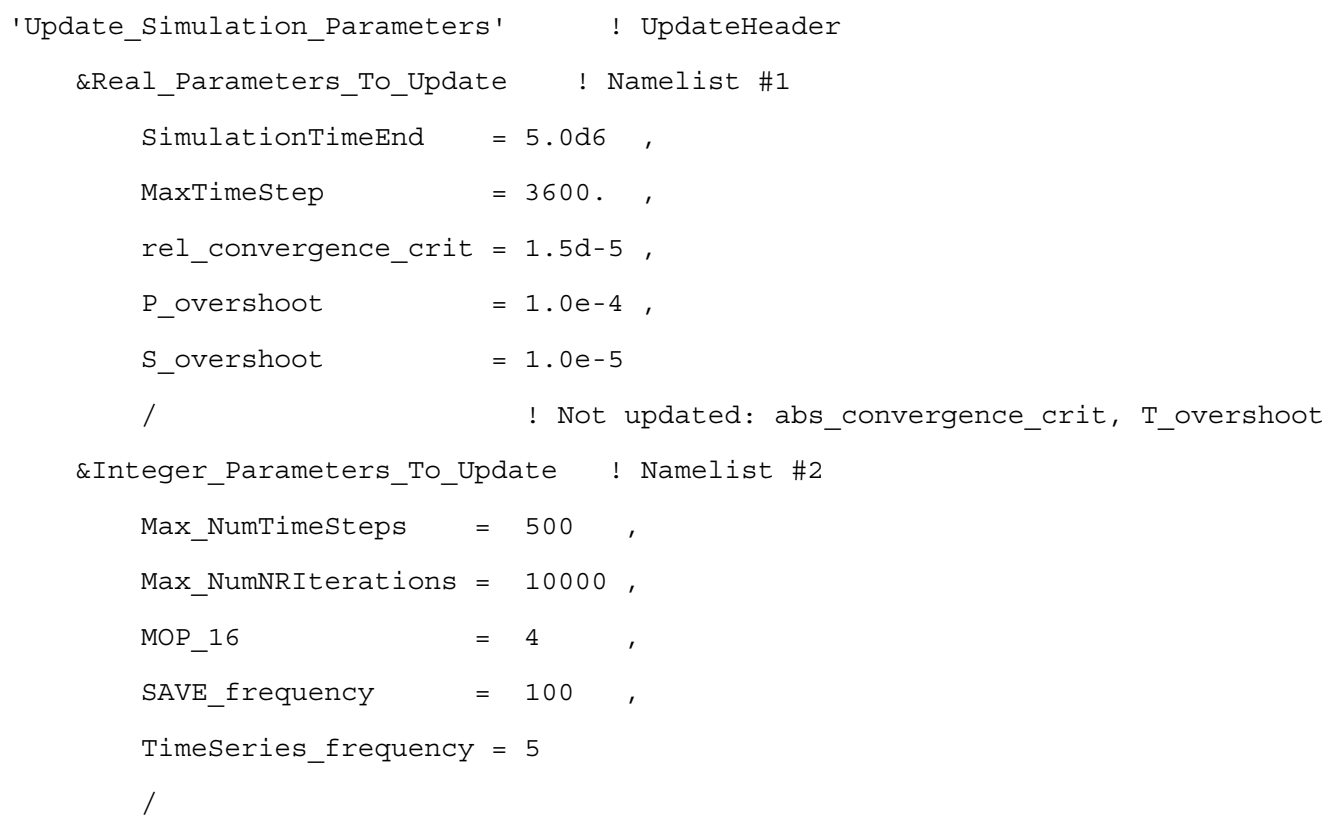

Figure 10.1. An example of a Parameter_Update_File for parameter updating in the course of a TOUGH+ simulation. Within the namelists (Real_Parameters_To_Update and Integer_Parameters_To_Update), parameters can be entered in any order, data are read using any kind of appropriate format, only the needed parameters are included, and comments can be added. 


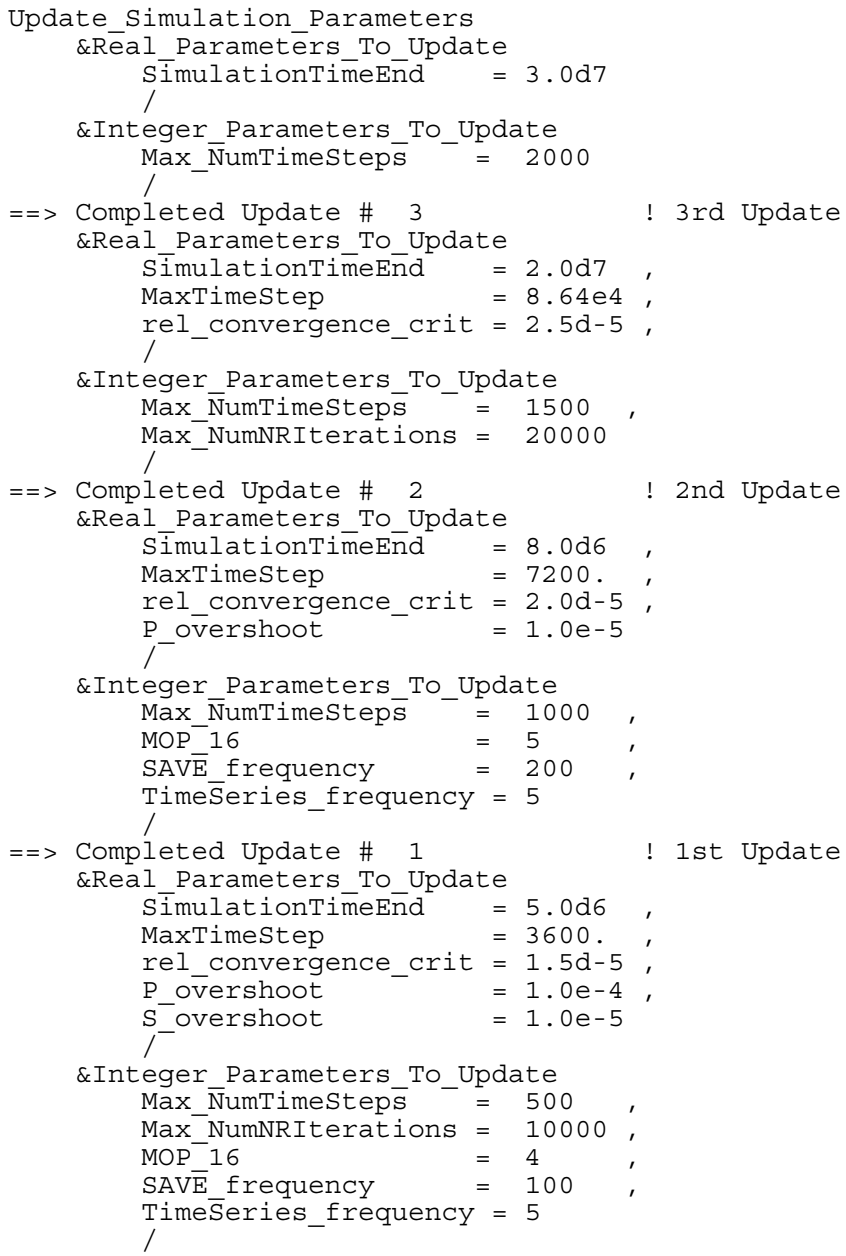

Figure 10.2. An example of a Parameter_Update_File indicating three completed parameter updates, in addition to another one (at the top of the file) that has not yet been executed. 


\subsection{Data Block SOLVR}

This (optional) block specifies parameters used by linear equation solvers.

$\underline{\text { Record SOLVR. } 1}$

Format(I1,2X,A2,3X,A2,2E10.4)

MatrixSolver, Z_preprocessing, O_preprocessing, Max_CGIterationRatio, CG_convergence_crit

MatrixSolver

Z_preprocessing

O_preprocessing
This integer variable selects the linear equation solver from among the following options:

$$
\begin{aligned}
& =1: \text { LUBAND } \\
& =2: \text { DSLUBC } \\
& =3: \text { DSLUCS } \\
& =4: \text { DSLUGM } \\
& =5: \text { DLUSTB }
\end{aligned}
$$

A character variable that determines the type of Z-preconditioning [Moridis and Pruess, 1998]. Regardless of user specifications, Z-preprocessing will only be performed when iterative solvers are used ( $2 \leq$ MatrixSolver $\leq 5)$, and if there are zeros on the main diagonal of the Jacobian matrix. The following options are available:

= ' Z0 ' : No Z-preprocessing; default for NumEqu $=1$ and MatrixSolver $=1$

= ' Z1 ': Replace zeros on the main diagonal by a small constant (1.e-25; default for NumEqu>1 and for $1<$ MatrixSolver $\leq 5$ )

= ' Z2 ': Make linear combinations of equations for each grid block to produce non-zero main diagonal entries

= ' Z3 ': Normalize equations, followed by Z2

= ' Z4 ' : Same as in O_preprocessing= ' 04 '

A character variable that determines the type of 
O-preconditioning [Moridis and Pruess, 1998].

$=$ ' $\bigcirc 0$ ': No O-preprocessing; default for

NumEqu $=1$ and MatrixSolver $=1$

= ' O1 ': Elimination of lower half of the maindiagonal submatrix with center pivoting

= ' O2': O1 + Elimination of upper half of the main-diagonal submatrix with center pivoting

= 'O3': 02 + Normalization; results in unit maindiagonal submatrices

= ' 04 ': Pre-processing which results in unit maindiagonal submatrices without center pivoting

Max_CGIterationRatioAn integer variable that specifies the maximum number of CG iterations as a fraction of the total number of equations $(0<$ Max_CGIterationRatio $\leq 1$; default is Max_CGIterationRatio $=0.1$ )

CG_convergence_crit A real variable that specifies the convergence criterion for the $\mathrm{CG}$ iterations (1.0e-12 $\leq$ CG_convergence_crit $\leq 1.0 e-6$; the default CG_convergence_crit $=1.0 e-6$ )

The solver DLUSTB implements the BiCGSTAB(m) algorithm [Sleijpen and Fokkema, 1993], an extension of the BiCGSTAB algorithm of van der Vorst (1992). DLUSTB provides improved convergence behavior when iterations are started close to the solution, i.e., near steady state. The preconditoning algorithms can cope with difficult problems in which many of the Jacobian matrix elements on the main diagonal are zero. An example was given in Pruess et al. [1991] in "two-waters" problems in which typically $2 / 3$ of the elements in the main diagonal are zero. Our tests show that this type of problem can be solved by means of Z2 or Z3 preconditioning [Moridis and Pruess, 1998]. 


\subsection{Discussion on Linear Equation Solvers}

The computational work to be performed in the course of a TOUGH+ simulation includes evaluation of thermophysical properties for all grid blocks, assembly of the vector of residuals and the Jacobian matrix, and solution of the linear equation system for each Newton-Raphson iteration step. Except for small problems with just a few grid blocks, the most computationally intensive of these different tasks is the solution of the linear equation system. TOUGH+ offers a choice of direct or iterative methods for linear equation solution; technical details of the methods and their performance can be found in Moridis and Pruess [1998].

The most reliable linear equation solvers are based on direct methods, while the performance of iterative techniques tends to be problem-specific and lacks the predictability of direct solvers. The robustness of direct solvers comes at the expense of large storage requirements and execution times that typically increase with problem size $\mathrm{N}$ (= number of equations solved) proportional to $\mathrm{N}^{3}$. In contrast, iterative solvers have much lower memory requirements, and their computational work will increase much less rapidly with problem size, approximately proportional to $N^{\omega}$, with $\omega \approx 1.4-1.6$ [Moridis and Pruess, 1995]. For large problems and especially 3-D problems with several thousand grid blocks or more, iterative conjugate gradient (CG) type solvers are therefore the method of choice.

The default linear equation solution technique in TOUGH + uses DSLUCS, a Lanczos-type bi-conjugate gradient solver, with incomplete LU-factorization as preconditioner. Users need to beware that iterative methods can be quite "fickle" and may fail for matrices with special features, such as many zeros on the main diagonal, large 
numerical range of matrix elements, and nearly linearly dependent rows or columns. Depending on features of the problem at hand, appropriate matrix preconditioning may be essential to achieve convergence. Poor accuracy of the linear equation solution will lead to deteriorated convergence rates for the Newtonian iteration, and an increase in the number of iterations for a given time step. In severe cases, time steps may fail with residuals either stagnating or wildly fluctuating. Information on the convergence of the linear equation solution is written to disk file LINEQ, which may be examined with any text editor. Users experiencing difficulties with the default settings are encouraged to experiment with the various solvers and preconditioners included in the TOUGH+ package. 


\section{Output Specifications}

In this section, the various primary and secondary variables that may be provided as output from TOUGH+ are discussed. In addition, data blocks are described for specifying output data, such as the times at which data are printed (block TIMES), a list of elements for which output data will be printed (ELEM_TIME_SERIES or FOFT), a list of connections for which data will be printed (CONX_TIME_SERIES or COFT), a list of sinks and sources for which data will be printed (SS_TIME_SERIES or GOFT), and whether to suppress version information (NOVER). All these $\mathrm{TOUGH}^{+}$output capabilities were available in the TOUGH2 family of codes [Pruess et al., 1991]. In addition, this section discusses new TOUGH + output capabilities that provide data describing the evolution of properties and conditions within a designated subdomain (subset) of the simulated domain (grid). This section also includes a few comments on error messages and warnings in the TOUGH+ family of codes. 


\subsection{Output of Primary and Secondary Variables}

The $\mathbf{T}+\mathbf{H}$ code can provide the following output:

1. The pressure, temperatures, saturations, and equilibrium pressure distributions.

2. The mass fractions of the various components in the various phases.

3. Fluxes (in $\mathrm{kg} / \mathrm{s}$ ) and velocities (in $\mathrm{m} / \mathrm{s}$ ) of the phases across the gridblock interfaces (connections) of the domain.

4. The primary variables and their changes in the elements of the domain.

5. Capillary pressures and relative permeabilities.

6. Densities, viscosities (when mobile), and enthalpies of the various phases.

7. Dissociation reaction rates and the corresponding heat of dissociation.

8. Volume and mass balances of the phases and components in the domain.

9. Production rates and production composition at wells.

10. Temporal variations of the most important variables at user-specified elements, connections and sources/sinks.

Of those possible outputs, (1), (8), (9), and (10) are always printed in the standard $\mathbf{T}+\mathbf{H}$ output. The amount of the output is controlled by the parameter Outputoption in the data block PARAM. In keeping with the TOUGH2 convention, if the OutputOption values are increased by 10 , printouts will occur after each iteration (not just after convergence).

$\mathbf{T}+\mathbf{H}$ also provides an output of the evolution of the gas hydrate reaction in the entire simulation domain during the simulation. The data are written to a file named Hydrate_Info, and include (a) time [days], (b) mass rate of gas release or reaction [kg $\mathrm{s}^{-1}$, (c) volumetric rate of gas release or reaction [standard $\mathrm{m}^{3} \mathrm{~s}^{-1}$ ], (d) cumulative mass of the released gas $[\mathrm{kg}]$, (e) cumulative volume of the released gas [standard $\mathrm{m}^{3}$ ], (f) cumulative volume of the free gas in the domain [standard $\mathrm{m}^{3}$ ], and $(\mathrm{g})$ remaining mass of hydrate in the reservoir $[\mathrm{kg}]$. 


\subsection{Data Block TIMES}

This optional block permits the user to obtain printout at specified times. This printout will occur in addition to printout specified in record PARAM. 1.

\section{Record TIMES. 1}

Format $(2 \mathrm{I} 5,2 \mathrm{E} 10.4)$

NumPrintTimes, Max NumPrintTimes, TimestepMax_PostPrint, PrintTimeIncrement

NumPrintTimes The number of times provided on records TIMES . 2, TIMES. 3, etc., (see below; restriction: NumPrintTimes $\leq 100$ ).

Max_NumPrintTimes The total number of times at which an output is desired

(NumPrintTimes $\leq$ Max_NumPrintTimes $\leq$ 100; default is NumPrintTimes = Max_NumPrintTimes).

TimestepMax_PostPrint The maximum time step size after any of the prescribed times have been reached (default is infinite).

PrintTimeIncrement Time increment for times with indices NumPrintTimes, NumPrintTimes $+1, \ldots$, Max_NumPrintTimes.

Record TIMES. 2, TIMES. 3, etc.

Format (8E10.4)

(PrintTime(i), $i=1$, NumPrintTimes)

PrintTime (i) A list of times (in ascending order) at which printout is desired. 


\subsection{Data Block Elem_Time_Series or FOFT}

This optional block introduces a list of elements (grid blocks) for which timedependent data are to be written out for plotting to a file called Elem_Time_Series during the simulation. If the keyword 'Elem_Time_Series' (or 'FOFT', thus maintaining compatibility with older input files developed for the TOUGH2 family of codes) is present in the input file, then the following data are read:

\section{Record ELEM SERIES. 1}

$$
\begin{aligned}
& \text { Format: } \begin{array}{l}
(\mathrm{A} 5,5 \mathrm{X}, \mathrm{A} 3) \text { for ElemNameLength }=5 \text {, or } \\
(\mathrm{A} 8,2 \mathrm{X}, \mathrm{A} 3) \text { for ElemNameLength }=8
\end{array} \\
& \text { ObsElemName (1), sum_flag }
\end{aligned}
$$

ObsElemName (1) The name of the first observation element. If ObsElemName $(1)={ }^{*} \mathbf{A L L}{ }^{*}$ ', then the output describes the evolution of mass and heat balance over the entire domain during the simulation period.

sum_flag If sum_flag $=$ 'sum ', then the output describes the evolution of mass and heat balance over the specified subdomain (composed of the elements ObsElemName ( $i$ ) listed in this data block)

Repeat for up to 100 elements $(\max \{i\}=100)$, entering ObsElemName (i) and sum_flag in each record.

$\underline{\text { Record ELEM SERIES. } 2}$

$$
\begin{aligned}
& \text { Format: } \begin{array}{r}
\text { (A5) for ElemNameLength }=5 \text {, or } \\
\text { (A8) for ElemNameLength }=8
\end{array} \\
& \text { (ObsElemName (i), } i=2,3,4, \ldots)
\end{aligned}
$$

ObsElemName ( i )

The name of the observation element. Repeat for up to 100 elements $(\max \{i\}=100)$.

Record ELEM SERIES. 3 
A blank record closes the Elem_Time_Series data block.

\subsection{Data Block Conx_Time_Series or COFT}

This optional data block introduces a list of connections for which time-dependent data are to be written out for plotting to a file called Conx_Time_Series during the simulation. If the keyword ' Conx_Time_Series' (or 'COFT', thus maintaining compatibility with older TOUGH2 input files) is present in the input file, then the following data are read:

\section{Record CONX SERIES. 1}

Format: $\quad(\mathrm{A} 10,5 \mathrm{X}, \mathrm{A} 3)$ for ElemNameLength $=5$, or $(\mathrm{A} 16,4 \mathrm{X}, \mathrm{A} 3)$ for ElemNameLength $=8$

ObsConxName (1), sum_flag

ObsConxName (1)

sum_flag
The name (= the ordered pair of the two constituent element names, i.e., the concatenation ConxName1// ConxName2 - see Section 7.3) of the first connection to be monitored. If ObsConxName $(1)=1>>$ ALL $<<$, then the output describes the evolution of mass and heat flows over the entire domain during the simulation period.

If sum_flag = 'sum', then the output describes the evolution of the sum of flows through the specified interface (defined by the list of connections in this data block)

$\underline{\text { Record CONX SERIES. } 2}$

$$
\begin{aligned}
& \text { Format: } \begin{aligned}
(\mathrm{A} 10,5 \mathrm{X}, \mathrm{A} 3) \text { for ElemNameLength }=5, \text { or } \\
(\mathrm{A} 16,4 \mathrm{X}, \mathrm{A} 3) \text { for ElemNameLength }=8
\end{aligned} \\
& \text { (ObsConxName }(\mathrm{k}), \mathrm{k}=2,3,4, \ldots)
\end{aligned}
$$


ObsConxName ( $i$ ) The name of the connection to be monitored.

Repeat for up to 100 connections $(\max \{\mathrm{k}\}=100)$.

Record CONX SERIES. 3

A blank record closes the Elem_Time_series data block.

The nine columns in the Conx_Time_Series_1T output file represent the following parameters describing mass and heat flows between the specified connections:

1. Time [sec]

2. Gas mass flow rate $[\mathrm{kg} / \mathrm{s}]$

3. Aqueous phase mass flow rate $[\mathrm{kg} / \mathrm{s}]$

4. Total heat transfer rate [W]

5. Volumetric flow rate of $\mathrm{CH}_{4}$ in the gas phase [standard $\mathrm{m}^{3} / \mathrm{s}$ ]

6. Volumetric flow rate of $\mathrm{CH}_{4}$ [standard $\mathrm{m}^{3} / \mathrm{s}$ ]

7. Cumulative volume of produced $\mathrm{CH}_{4}$ [standard $\left.\mathrm{m}^{3}\right]$

8. Cumulative mass of produced $\mathrm{H}_{2} \mathrm{O}$ [in kg]

9. Mass fraction of inhibitor in the flow (production) stream

The data in columns 2 to 8 represent cumulative quantities across the list of connections provided in the data block Conx_Time_Series (or COFT). If one of the cells in the connection represents a constant-pressure boundary (i.e., in the case of constant-pressure production), then the data in this file provide a means of evaluation of the gas production potential of a hydrate deposit. Note that the data in the Conx_Time_Series file refer to gas production regardless of origin because it is not possible to determine the provenance of the gas flowing across these interfaces and to reliably differentiate between gas from hydrate dissociation and pre-existing "free" gas in the reservoir. 


\subsection{Data Block SS_Time_Series or GOFT}

This optional data block introduces a list of sinks/sources for which time-dependent data are to be written out for plotting to a file called SS_Time_Series during the simulation. If the keyword 'SS_Time_Series' (or 'GOFT', to allow backward compatibility with older TOUGH2 input files) is present in the input file, then the following data are read:

Record SS SERIES.1

Format (A5)

(ObsSSName (i), $i=1,2,3, \ldots$ )

ObsSSName (i) Name of the sink or source to be monitored. This should be among one of the names SS_name in the GENER data block - see Section 9.1.

Repeat for up to 100 sinks/sources $(\max \{i\}=$ 100), one per record. When no sinks or sources are specified here, by default tabulation will be made for all.

\section{Record SS_SERIES.2}

A blank record closes the SS_Time_Series data block.

The eight columns in the SS_Time_Series_1T output file represent the following parameters describing mass and heat flows through the sources or sinks:

1. Time [sec]

2. $\mathrm{CH}_{4}$ mass flow rate of the sources $/$ sinks $[\mathrm{kg} / \mathrm{s}]$

3. $\mathrm{CH}_{4}$ volumetric flow rate of the sources/sinks [standard $\mathrm{m}^{3} / \mathrm{s}$ ]

4. Cumulative mass of produced $\mathrm{CH}_{4}[\mathrm{~kg}]$

5. Cumulative volume of produced $\mathrm{CH}_{4}$ [standard $\mathrm{m}^{3}$ ]

6. Water mass flow rate of the sources $/$ sinks $[\mathrm{kg} / \mathrm{s}]$

7. Cumulative mass of produced $\mathrm{H}_{2} \mathrm{O}[\mathrm{kg}]$

8. Mass fraction of inhibitor in the production stream 
When sinks (i.e., wells) are involved, these can provide a means for the evaluation of the gas production potential of a hydrate deposit.

\subsection{Data Block SUBDOMAINS}

This optional data block is a new TOUGH+ feature. It allows monitoring the evolution of the pore volume-averaged properties and conditions in a set of subdomains (subsets) of the global grid (domain). The time series results are written in a number of separate output files that are equal to the number of the subdomains, and are named according to the convention $\mathbf{x} \mathbf{x} \mathbf{x} \mathbf{x} \mathbf{x}$-Time_Series, where ' $\mathbf{x} \mathbf{x} \mathbf{x} \mathbf{x} \mathbf{x}$ ' is the name of the subdomain under observation. If the keyword SUBDOMAINS ' is present in the input file, then the following data are read using NAMELIST formats:

\section{Record SUBDOMAIN . 1}

This record includes general data describing the number of subdomains to be monitored and the frequency of recording the results. The namelist in this record is named Real_Parameters_To_Update, and includes the following parameters:

Subdomain_General_Info: An integer variable describing the number of subdomains on which time series data are to be obtained. There is no upper limit in the number of subdomains that can be defined.

NOTE: Although, technically-speaking, namelists allow easy omission of any parameter within it, the Subdomain_General_Info variable must be set to a positive value for the simulation to continue.

subdomain_output_frequency: An integer variable specifying the recording frequency of the subdomain data in the time series. The default value is 1 . 
The structure of the Real_Parameters_To_Update namelist (and its use as an input format in the data block SUBDOMA $\bar{I} \mathbf{N}$ ) is best illustrated in the example of Figure 11.1.

\section{Record SUBDOMAIN . 2}

The namelist in this record provides general information on the subdomain. It is named Individual_subdomain_Specifics and includes the following parameters:

subdomain_name

The subdomain name is described by this character variable of a maximum of 8 characters. Upon reading the variable, the $\mathrm{TOUGH}+$ code creates an

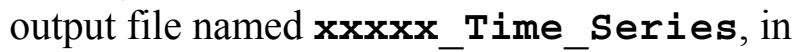
' $\mathbf{x x x x x}$ ' is the value of subdomain name. If this variable is omitted in the input, then the TOUGH+ code assigns internally the name SubDomNNN, where ' NNN' is the subdomain number in the entry sequence.

number_of_regions An integer variable specifying the number of regions of which the subdomain is composed.

NOTE: The number_of_regions variable must be set to a positive value for the simulation to continue.

The structure of the Individual_Subdomain_Specifics namelist (and its use as an input format in the data block SUBDOMAIN) is best illustrated in the example of Figure 11.1.

\section{Record SUBDOMAIN . 2 . 1}

The namelist in this record is named Region_Specifics. It provides information that allows the definition of the region within the subdomain and identification of the elements belonging to it, and includes the following parameters:

definition_mode

This character variable describes the method by which the region is defined. In the current version of TOUGH+, the only available option is definition_mode = 'Geometry', indicating that the region within the subdomain is to be defined through its geometric boundaries. 
region_shape

This character variable describes the shape of the region that is about to be defined. The following self-explanatory options are available:

= 'Rectangle': This option can only be used in Cartesian grids, i.e., if coordinate_system = 'Rectangle'.

$=$ 'Cylinder': This option can only be used for either Cartesian or cylindrical grids.

= 'Sphere ' : This option can only be used for either Cartesian or cylindrical grids.

If region_shape $=$ 'Rectangle', then the following variables must be included in the namelist:

Xmin, Xmax, Ymin, Ymax, Zmin, Zmax

These real variables indicate the range (minimum and maximum) of the region along the $x$-, $y$ - and $z$-axis of the Cartesian coordinate system, respectively.

If region shape $=$ 'Cylinder' and coordinate system $=$ ' Cylindrical', then the following variables must be included in the namelist:

Rmin, Rmax, Zmin, Zmax

These real variables indicate the range (minimum and maximum) of the region along the $r$ - and $z$-axis of the Cartesiancylindrical coordinate system, respectively.

If region_shape $=$ ' Cylinder' and coordinate_system $=$ 'Cartesian', then the following variables must be included in the namelist:

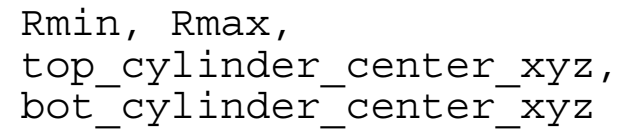

The real variables Rmin and Rmax are the minimum and maximum radius of the cylindrical region. The real, 1D arrays top_cylinder_center_xyz and bot_cylinder_center_xyz have a dimension of 3. These are the $(x, y, z)$ coordinates of the centers of the top and bottom circular surfaces of the cylinder, respectively.

If region_shape $=$ 'Sphere', then the following variables must be included in the namelist:

$$
\text { Rmin, Rmax, sphere_center_xyz }
$$


The real variables Rmin and Rmax are the minimum and maximum radius of the sphere. The $(x, y, z)$ coordinates of the center of the sphere are stored in the elements of the real, 1D array top_cylinder_center_xyz of dimension 3. Of those three values, only the $3^{\text {rd }}$ (corresponding to the $z$-coordinate of the center of the sphere) one is used when coordinate_system = 'Cylindrical'.

Thus, its is possible to combine regions of very different geometries to create subdomains that are very irregular in shape.

NOTE \#1: All the various variable combinations and permutations in namelist Region_Specifics must be defined for the simulation to continue.

NOTE \#2: All the regions within the subdomain must be defined. A total of number of regions records SUBDOMAIN.2.1 (one for each region of the subdomain) must be provided. Thus, if we define two subdomains, the first with 3 regions and the second with 4, then we need to provide the following data:

For the $1^{\text {st }}$ Subdomain: One SUBDOMAIN. 2 record, and 3 SUBDOMAIN. 2.1 records. For the $2^{\text {nd }}$ Subdomain: One SUBDOMAIN 2 record, and 4 SUBDOMAIN. 2.1 records.

The structure and use of the Region_Specifics namelist in the data block SUBDOMAIN is best illustrated by the example of Figure 11.1.

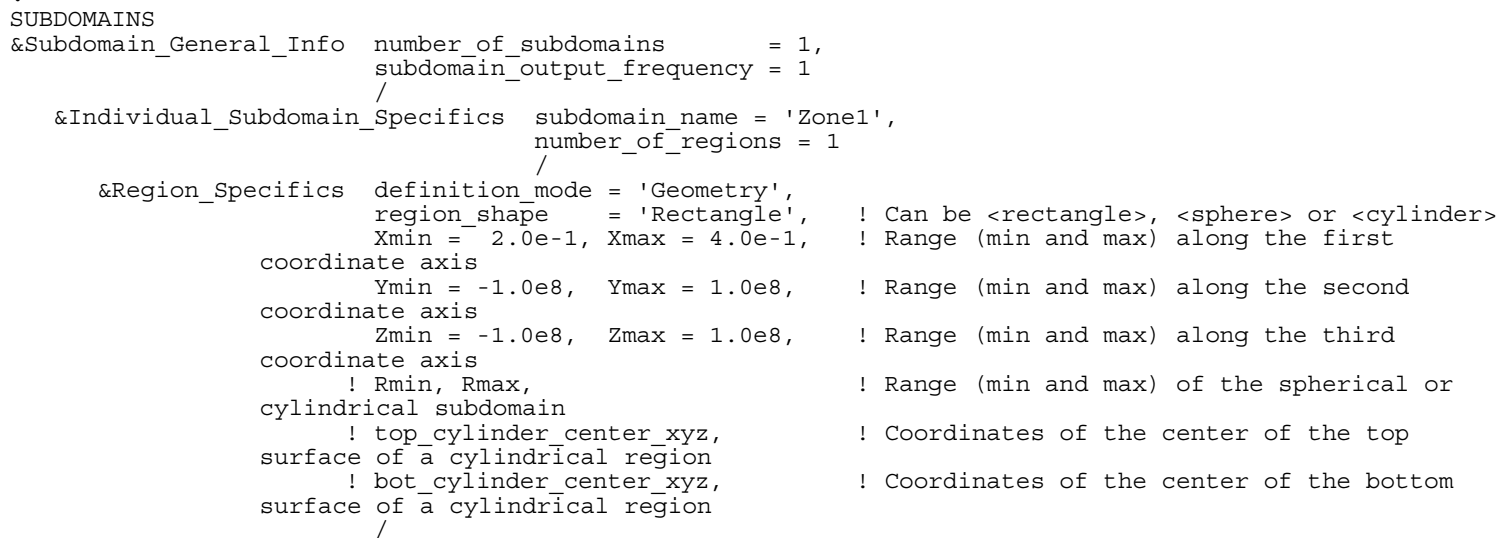

Figure 11.1. An example of the SUBDOMAINS data block for tracking the evolution of volumeaveraged properties and conditions in specified subdomains. This data block uses namelistbased formats for data inputs. 


\subsection{Data Block NOVER}

One record with NOVER typed in columns 1-5 will suppress printing of a summary of versions and dates of the program units used in a TOUGH+ run.

\subsection{Warning Output and Error Messages}

If inputs indicate conflicting conditions and/or parameter values are outside realistic ranges, TOUGH + is designed to respond according to the severity of the violation. Noncritical conflicts result in a warning or clarifying message, internal adjustment of the corresponding conditions and/or parameters, and continuation of the simulation. Serious violations result in an error message identifying the problem, and the simulation is aborted. 


\subsection{Example Problems}

\subsection{Example Files and Naming Conventions}

The files corresponding to the examples discussed in this manual can be found in the directory "TestRuns" in the CD accompanying this manual. The input files of the example problems have the general name Test_XXX (where XXX is an identifier) and are the following:

1. Test_1T

2. Test_1Tk

3. Test_1P

4. Test_1Pk

5. Test $1 \mathrm{P}$ ice

6. Test_1T̄bs

7. Test_1TbSk

8. Test_1TSk

9. Test_2Qp

10. Test_3T

11. Test_3Qp

12. Test_3Qpk 
13. Test_RadH1

14. Test_2D

The corresponding output files are also included in the directory "TestRuns". The naming convention of the generic TOUGH+ output files involves the suffix ".out" at the end of the input file name. The hydrate-specific output files have the general name Hydrate_Info_XXX, where XXX is the identifier of the input file name.

For some examples in which the hydration reaction (dissociation or formation) is induced by boundary conditions, the additional output files of the time series at the boundary connection are included under the general name Conx_Time_Series_xxx. Similarly, if the hydration reaction is induced by sources or sinks, the corresponding additional output file of the time series of the conditions at the sinks and/or sources is included in the directory "TestRuns" under the name SS_Time_Series_xxx.

\subsection{Problem Test_1T: Thermal Stimulation, Equilibrium Dissociation, No Inhibitor}

This 1-D problem is designed to demonstrate the basic concepts of hydrate dissociation by means of thermal stimulation. Note that it is practically impossible to separate the thermal and pressure processes in the course of dissociation, regardless of the dissociation method. As hydrate dissociates in thermal stimulation, gas evolves and accumulates in porous media, leading to pressure increases immediately ahead of the hydrate interface that shift the hydrate toward the stability zone. Conversely, depressurization is a very effective method of hydrate dissociation, the endothermic 
nature of which results in rapid temperature drop and enhanced stability of the remaining hydrate.

For convenience, the input file is listed in Figure 12.1. As an exercise, a novice user is urged to identify the various variables and parameters in the input file.

The 1-m long domain (represented by 10 active cells of uniform $\Delta x=0.1 \mathrm{~m}$ size) is a pressurized and thermally insulated column of a porous medium, in which hydrate and water coexist at a pressure of $4.0 \times 10^{6} \mathrm{~Pa}$ and $T=1.2{ }^{\circ} \mathrm{C}$, i.e., well within the hydrate stability zone. At a time $t=0$, the boundary at $x=0$ becomes permeable and the column comes in contact with warm water at a temperature of $T_{b}=45^{\circ} \mathrm{C}$. As the temperature in the column increases, hydrate dissociation is expected to occur, leading to the evolution of gas, which will then escape the column by flowing through the high-temperature boundary. Note that the pressure in the high-temperature boundary is equal to the initial one in the column, thus allowing escape of the pressurized gas. In this simulation, the hydrate dissociation reaction is assumed to occur at equilibrium. Under these conditions, dissociation is limited and controlled by heat transfer issues.

The porous medium has a porosity $\phi=0.3$, and a permeability $k=2.96 \times 10^{-13} \mathrm{~m}^{2}(=$ $30 \mathrm{mD}$ in oilfield units). In the presence of the ice and hydrate solid phases, the critical mobile porosity (i.e., the "free" porosity -- not occupied by solids -- below which the porous medium becomes impermeable) is 0.05 , and the porosity reduction exponent is 3 .

Note that a non-zero (and relatively significant) pore compressibility $\left(=10^{-8} 1 / \mathrm{Pa}\right)$ is assigned to the porous medium. This is necessary in hydrate simulations, in which evolution of solid phases of lower density (such as ice and hydrate) can lead to extraordinarily high pressures as the aqueous phase disappears if pore compressibility is 
small. In this illustrative example, the thermal conductivity $(=3.1 \mathrm{~W} / \mathrm{m} / \mathrm{K})$ is relatively large, but was given this value to enhance heat conduction (the main heat transfer mechanism from the high-temperature boundary).

The hydrate properties and the type of the reaction are listed in the HYDRATE data block. The thermal conductivity, specific heat and density of the $\mathrm{CH}_{4}$ hydrate are from data in Sloan [1998], and are constants because no information is available on their dependence on temperature and/or pressure. However, $\mathbf{T}+\mathbf{H}$ assumes that the constant input density of the $\mathrm{CH}_{4}$ hydrate is that at the quadruple point, and the hydrate density in the simulations is internally adjusted by assuming that its compressibility and thermal expansivity are the same as those of ice (as calculated within $\mathrm{TOUGH}+$ ). The inhibitor data correspond to the properties of $\mathrm{NaCl}$.

Test_1T.out, the standard TOUGH+ output corresponding to the input file Test_1T, can be found in the directory "TestRuns" in the CD accompanying this manual. Because MOP $(5)=3$, the output includes detailed messages about the evolution of the residuals during the Newtonian iterations at each time step, as well as about phase changes occurring during the course of the simulations. Because Outputoption $=3$, a full output is obtained that provides a very detailed list of the conditions, parameters and thermophysical properties of the system at each cell and at each connection. Thus, the output describes the pressure, temperature, phase saturation, partial $\mathrm{CH}_{4}, \mathrm{H}_{2} \mathrm{O}$-vapor pressure, equilibrium hydration pressure, inhibitor mass fraction in the aqueous phase, $\mathrm{CH}_{4}$ concentrations in the aqueous and gas phases, phase densities, porosity, capillary pressure, relative permeability of the gas and aqueous phases, heat and fluid fluxes, mobile phase velocities, $\mathrm{CH}_{4}$ fluxes in the aqueous and gas phases, primary 
variables and their changes, phase enthalpies, rate of the hydration reaction, heat of the hydration reaction, and the temperature shift in the hydrate P-T equilibrium caused by the presence of the inhibitor. Additionally, the output provides mass and volume balances of the phases, component mass balances, and component distribution into the phases.

The results in the portion of the output in the Test_1T.out file (see Appendix A) are consistent with the expected system response. The hydrate dissociation front is observed to move deeper into the column with time, and is in the eighth cell at the end of the simulation period $\left(55^{\text {th }}\right.$ timestep). As temperature increases, hydrate begins to dissociate (see the gradual hydrate saturation decline and eventual disappearance in the warming cells, along with an emerging and then increasing gas saturation). The maximum pressure is observed at the dissociation front, indicated by the first incidence of coexistence of aqueous, gas and hydrate phases in a cell (and immediately ahead of a cell from which all hydrate has disappeared). This higher pressure is caused by the gas evolution in response to hydrate dissociation, and leads to formation of additional hydrate (indicated by higher hydrate saturations and a positive hydrate reaction rate) in the cell immediately in front of the one that contains the dissociation front. As expected, the gas fluxes indicate gas flow toward the high-temperature permeable boundary. Note that, because the maximum pressure occurs at the hydrate dissociation front, gas flows in both directions from the cells that contain the hydrate interface. Comparison of the partial pressure of $\mathrm{CH}_{4}$ to the hydrate equilibrium pressure confirms that dissociation occurs at equilibrium.

The additional output file Hydrate_Info_1T (also listed in Appendix A) provides a measure of the cumulative dissociation behavior in the entire system, as opposed to that 
in individual cells (described in the standard TOUGH + output). This output file features self-explanatory headings, and includes the instantaneous cumulative rates of $\mathrm{CH}_{4}$ gas evolution from hydrate (expressed as mass rates or standard volume rates), as well as the corresponding cumulative amount (in terms of total mass and total standard volume) of hydrate-originating $\mathrm{CH} 4$. Thus, it provides a measure of the overall performance of the hydrate-bearing system as a gas production source. Negative rates and masses/volumes indicate hydrate generation. Note that the results in any Hydrate_Info output indicate conditions and response within the accumulations, and do not necessarily reflect behavior and performance at the production well. Thus, for successful exploitation of a hydrate accumulation, significant $\mathrm{CH} 4$ volumes must be released through dissociation within the deposit, but the gas must also be able to be removed from the system through an appropriate collection (i.e., well) facility. 
"Test_1T": Equilibrium hydrate dissociation/Thermal Stimulation MEMORY

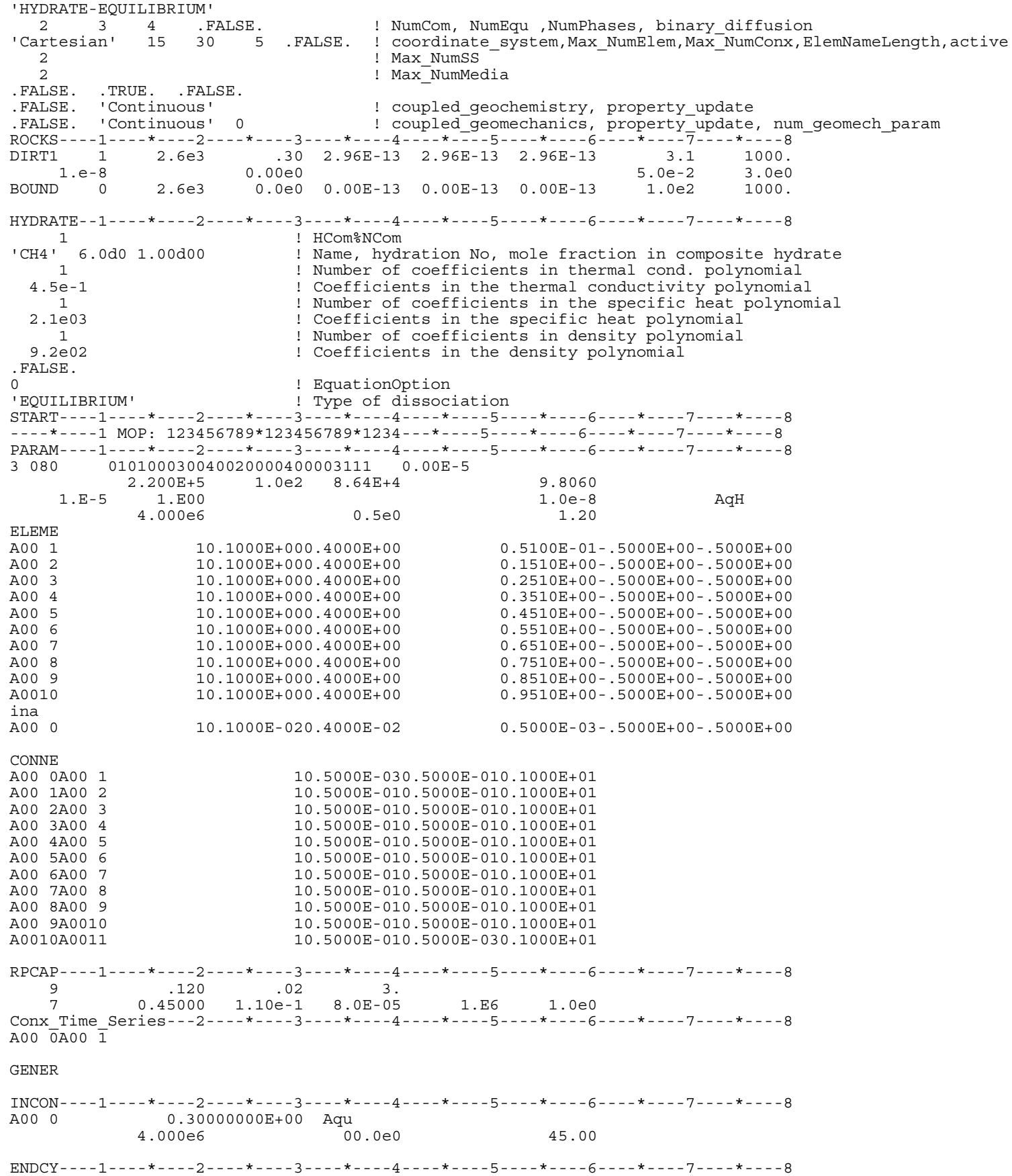

Figure 12.1. Input file for example problem Test_1T (in Section 12.2) involving thermal stimulation, equilibrium dissociation, and no inhibitor. 


\subsection{Problem Test_1Tk: Thermal Stimulation, Kinetic Dissociation, No Inhibitor}

This 1-D problem is entirely analogous to that discussed in Test_ $1 \mathrm{~T}$, from which it differs only in that hydrate dissociation is not treated as an equilibrium reaction but as a kinetic reaction. For convenience, the input file Test_1Tk is listed in Figure 12.2. It can be seen that the inputs are identical, with the exception of parameters and values describing the kinetic nature of the reaction.

Because a kinetic reaction confers an additional degree of freedom, the number of mass components NumCom increases by one to NumCom $=3$ (the hydrate is now a mass component in addition to being a solid phase, see discussion in Section 2.2). Consequently, the number of equations per cell increases by one to NumEqu $=4$, the number of primary variables describing initial conditions (in data blocks PARAM and INCON) increases by one, and the corresponding Jacobian matrix is larger than the one in problem Test_1T. As an exercise, a novice user is urged to identify the primary variables in this input file, and to differentiate them from those in the input file Test_1T.

Both the EOS_Name heading in the MEMORY block (record MEMORY. 2), and in the Reaction_Type variable in the HYDRATE data block (record HYDRATE.11) indicate that this is kinetic system. The values of the kinetic parameters of the hydration reaction (i.e., intrinsic reaction rate and the activation energy) were obtained from Kim et al. (1987), and Clarke and Bishnoi (2002). The area adjustment factor (variable Area_Factor in record HYDRATE. 12 (also see equation 2.6) was assumed to be equal to one. 
The interested user is directed to the directory "TestRuns" for review of the standard $\mathrm{TOUGH}+$ output, as well as of the additional Hydrate_Info_1Tk and Conx_Time_Series_1Tk output files. Upon inspection, a general observation that can be made is that this is a more difficult problem to solve, and that it takes more timesteps (65 vs. 54 ) to cover the simulation period than in problem Test_1T. A reason for the slower time advance is the kinetic hydrate dissociation, which imposes limits on the time step size.

The results of the illustrative problems in Test_1T and Test_1Tk are compared in Figures 12.3 to 12.6. It should be noted that the results of these two simulations are affected by the relatively coarse discretization in the two simple systems, but conclusions drawn from the relative performance are valid and provide a robust perspective in the evaluation of such simulation results.

Figure 12.3 shows the cumulative rates of $\mathrm{CH}_{4}$ release from the dissociation of hydrate in the columns of problems Test_1T and Test_1Tk. The effect of the relatively coarse discretization is evident in the oscillatory nature of the curves of rate evolution over time. An increase in the rate of $\mathrm{CH}_{4}$ release is a direct consequence of faster dissociation, which leads to a localized increase in pressure (caused by the evolving gas and the resistance to flow posed by the porous medium) and a drop in temperature (caused by the endothermic nature of the hydrate reaction). The increase in pressure and temperature drop result in a subsequent reduction in the rate of dissociation until conduction and convection (of the advancing warm water) can raise the temperature and accelerate dissociation. The result of this sequence of events is the (roughly) periodic nature of the rate curve (involving the repetition of a similar pattern), which is 
substantially affected by the size of the cells. Thus, each of the four distinct patterns in Figure 12.3 corresponds to a different cell, and represents the advance of the hydrate dissociation front through that cell at the time of observation.

A significant observation that can be drawn from Figure 12.3 is that the equilibrium and kinetic rates are different (with the kinetic rate lower) when the dissociation front first moves into a cell, but practically coincide afterwards. However, at the very beginning of the simulation, a burst of very rapid gas release is observed in the case of kinetic dissociation. This pattern of behavior is consistent with expectations because the maximum deviation between kinetic and equilibrium predictions is anticipated to occur at the maximum deviation from equilibrium. This is followed by equilibrium dissociation along the $\mathrm{L}+\mathrm{G}+\mathrm{W}$ phase boundary. The coincidence of the kinetic and equilibrium rates indicates that equilibrium is established very rapidly, as dictated by the relatively fast intrinsic dissociation rate and the high temperature. In cases of thermal stimulation involving high temperatures, equilibrium and kinetic dissociation models are expected to give very similar results (in terms of overall gas release from the hydrate) because (a) the high intrinsic reaction rate does not pose a kinetic limitation on the reaction, and (b) the reaction rate is dominated by heat transfer issues.

In Figure 12.4, the cumulative gas volumes released from the hydrate in the two problems are very similar, and confirm the observations from the discussion on the rates. The discretization effects are evident in the distinctly "segmented" appearance of the curves, which exhibit "bumps" corresponding to the periodic rate patterns in Figure 12.3. Note that Figures 12.3 and $\mathbf{1 2 . 4}$ were plotted using the data from the output files Hydrate_Info_1T and Hydrate_Info_1Tk. 
In problems Test_1T and Test_1Tk, gas is produced as it escapes into the warm water across the $x=0$ boundary. Figures 12.5 and 12.6 (based on data from the Conx_Time_Series_1T and Conx_Time_Series_1Tk output files) show the volumetric production rate of $\mathrm{CH}_{4}$ and the cumulative $\mathrm{CH}_{4}$ production. In these two examples, all the $\mathrm{CH}_{4}$ originates from the dissociation of the hydrate because no 'free' $\mathrm{CH}_{4}$ was initially present in the system. Figure $\mathbf{1 2 . 5}$ shows the same periodic pattern observed in Figure 12.3, denoting the effect of discretization. The $\mathrm{CH}_{4}$ production rates (i.e., the fluxes across the $x=0$ boundary) are initially the same, but then the kinetic rate decreases (relative to the equilibrium rate). This difference occurs as the hydrate dissociation front is about to move into an adjacent cell. The cumulative effect is reflected in the total gas production (shown in Figure 12.4, and measured as the total $\mathrm{CH}_{4}$ volume that crosses the $x=0$ boundary), which indicates lower gas production for kinetic dissociation. The effect of discretization is apparent in the "segmented appearance" of the gas production curves, which correspond directly to the periods in the production rates in Figure 12.5. 
"Test 1Tk": Kinetic hydrate dissociation/Thermal stimulation MEMORY

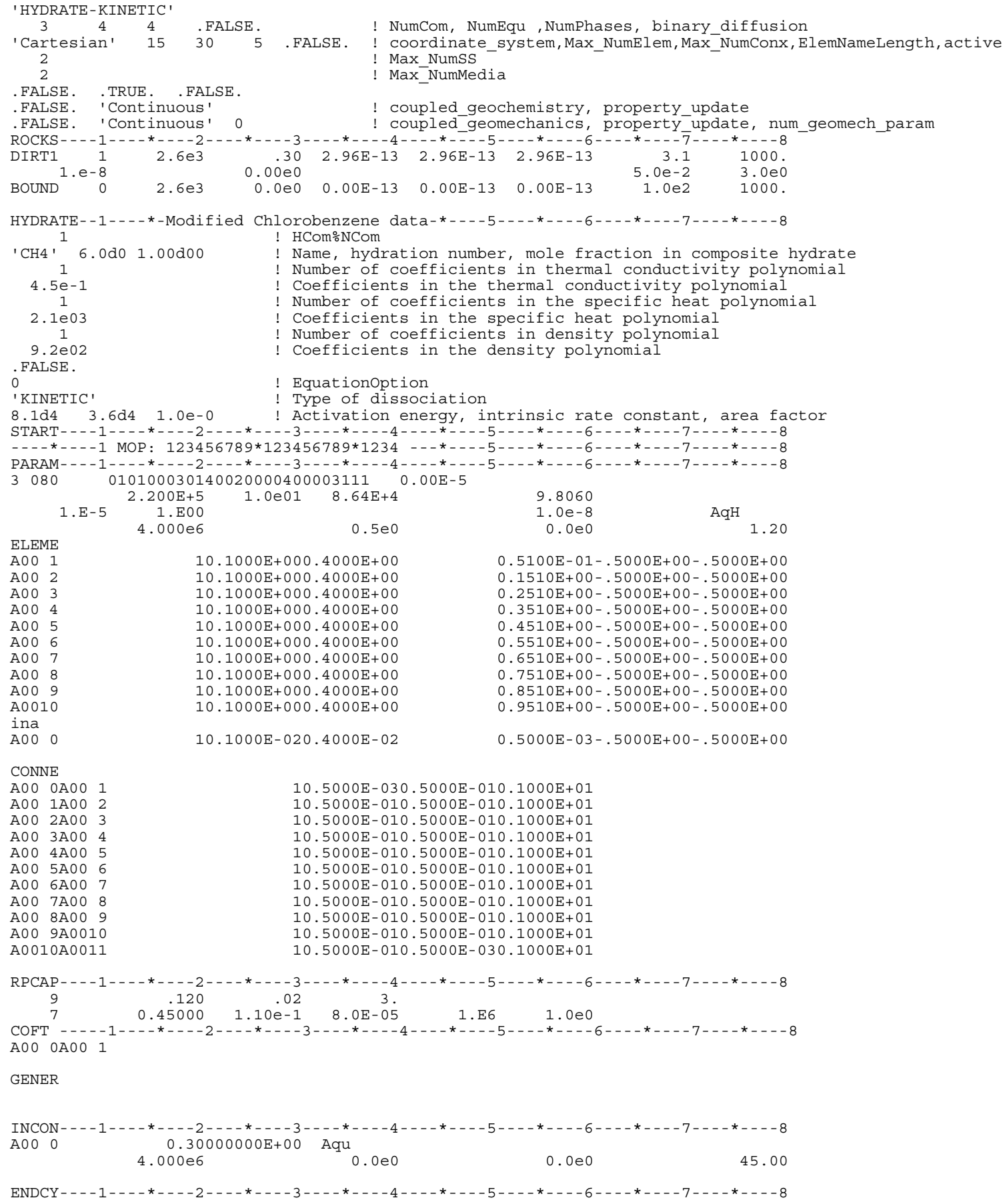

Figure 12.2. Input file for example problem Test_1Tk (in Section 12.3) involving thermal stimulation, kinetic dissociation, and no inhibitor. 


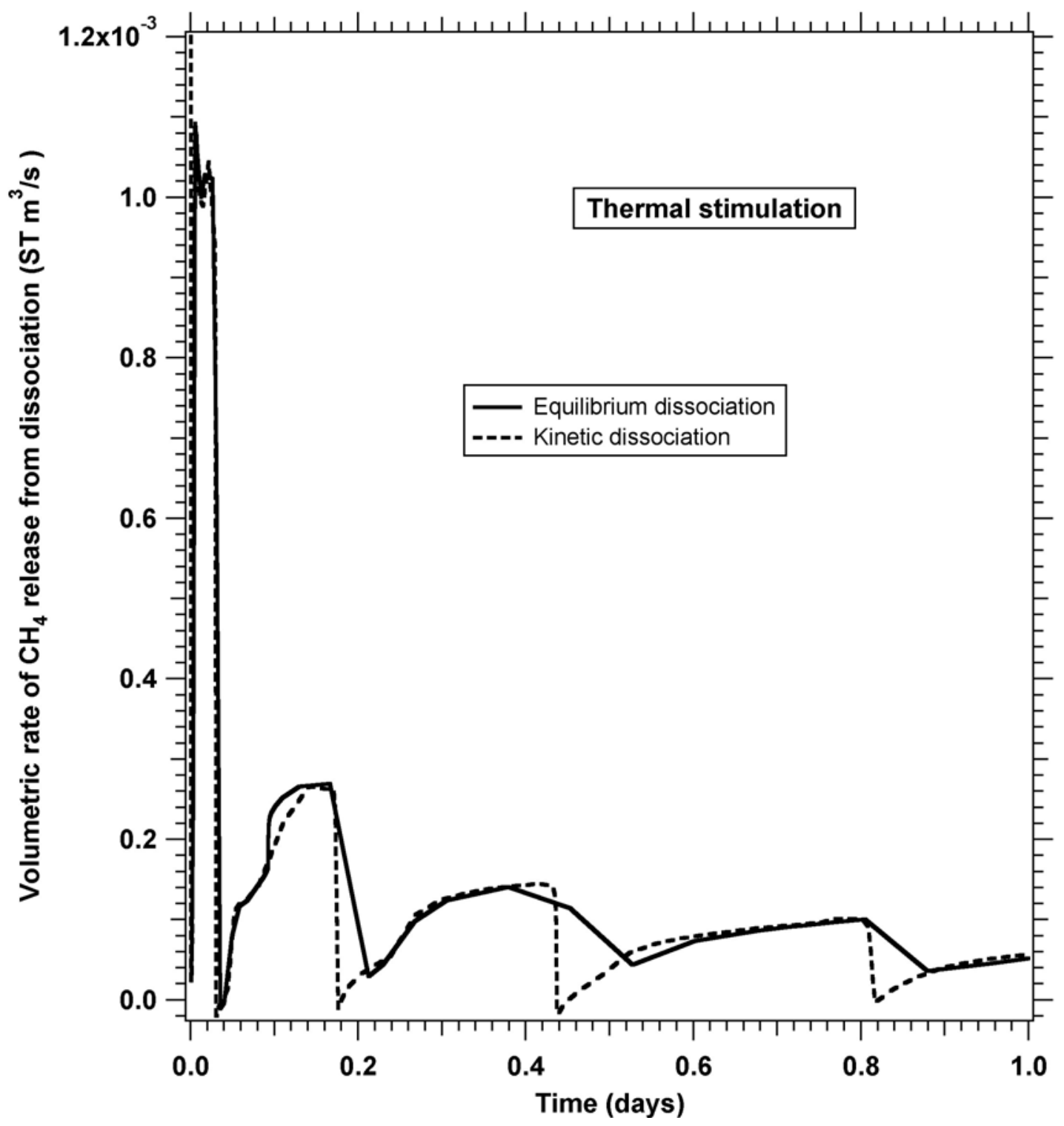

Figure 12.3. Comparison of the volumetric rates of $\mathrm{CH}_{4}$ release from hydrate dissociation in problems Test_1T and Test_1Tk. 


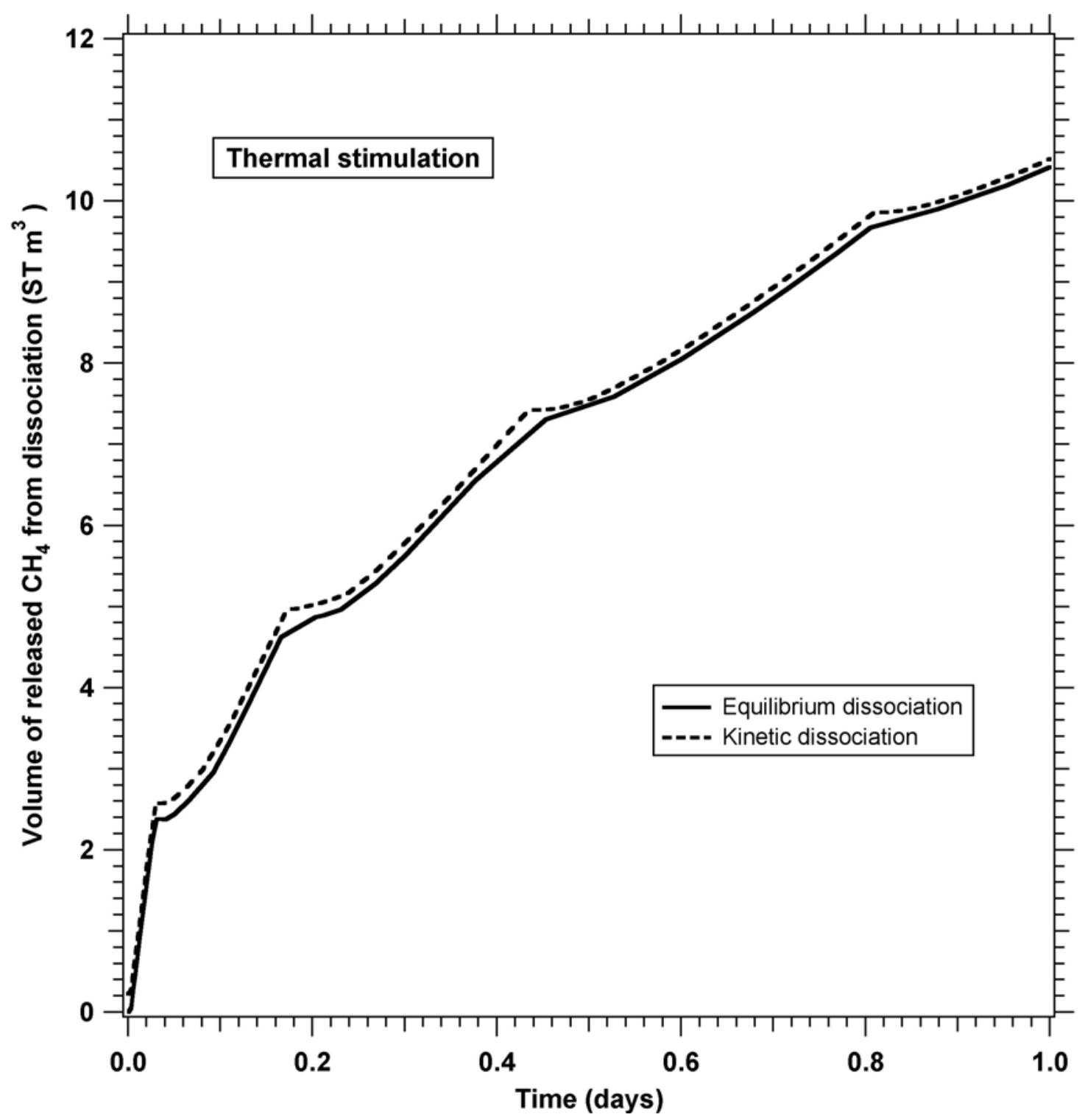

Figure 12.4. Comparison of the cumulative volumes of $\mathrm{CH}_{4}$ released from hydrate dissociation in problems Test_1T and Test_1Tk. 


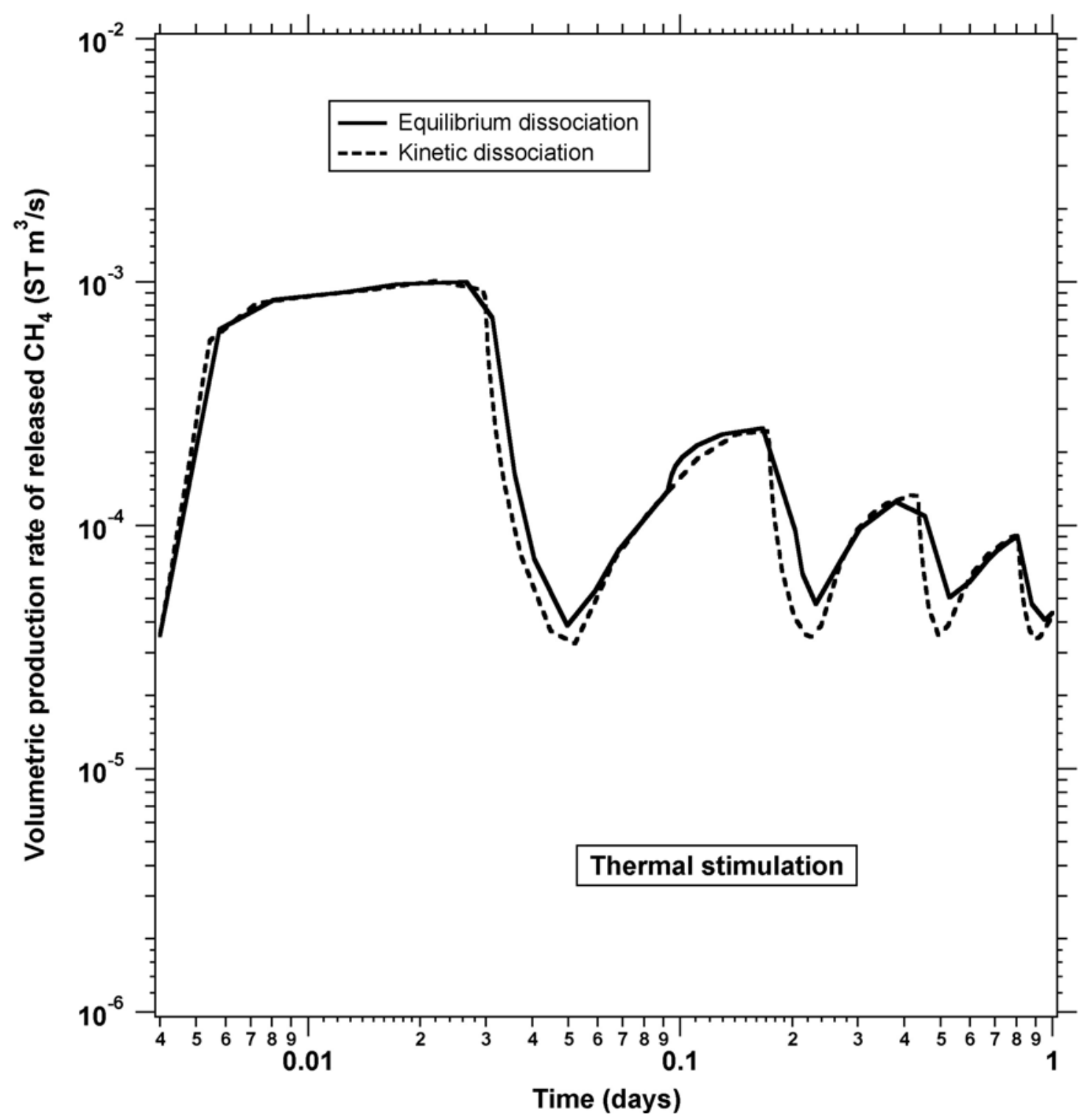

Figure 12.5. Comparison of the volumetric production rates of $\mathrm{CH}_{4}$ (measured as the flux crossing the $x=0$ boundary) in problems Test_1T and Test_ $1 \mathrm{Tk}$. 


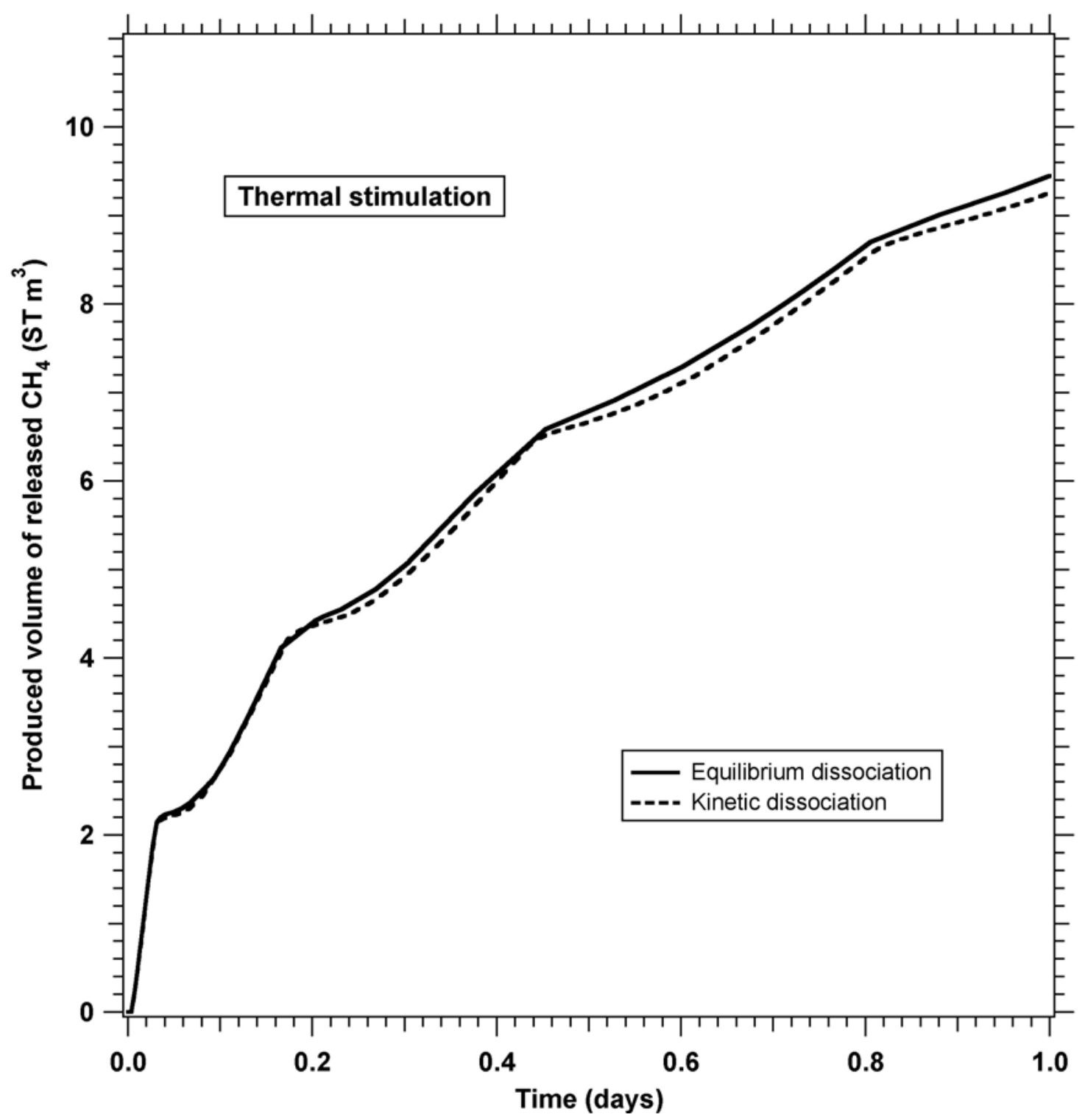

Figure 12.6. Comparison of the total production volumes of $\mathrm{CH}_{4}$ (measured as the total volumes crossing the $x=0$ boundary) in problems Test_1T and Test_1Tk. 


\subsection{Problems Test_1P: Depressurization, Equilibrium Dissociation, No Inhibitor}

The only difference between the two-phase (hydrate and aqueous) 1-D problem in Test_1P and that discussed in Test_1T is in the boundary conditions at $\mathrm{X}=0$. Instead of a high-temperature permeable boundary (leading to thermal dissociation), the $\mathrm{x}=0$ boundary is now kept at a pressure of $P_{b}=2.7 \times 10^{6} \mathrm{~Pa}$, which is lower than the initial pressure in the remainder of the column $\left(P=6 \times 10^{6} \mathrm{~Pa}\right)$. The pressure differential between the column and boundary and the non-zero permeability of the connection joining the two allow depressurization, which in turn induces dissociation. Note that the boundary pressure $P_{b}$ is larger than the pressure at the quadruple point of $\mathrm{CH}_{4}$-hydrate. This allows dissociation without reaching the quadruple point, which consequently keeps ice from forming. The constant boundary temperature of $T_{b}=1{ }^{\circ} \mathrm{C}$ is not expected to play a significant role in dissociation, despite its being above the melting point, because heat convection (fueled by fluid flow) is the main means of heat transfer, with conduction being a slow and inefficient process.

In the absence of an external heat source, the strongly endothermic reaction of hydrate dissociation is fueled by the heat provided by its surroundings. Consequently, temperature is expected to decline rapidly as dissociation proceeds.

The input file for the problem Test_1P appears in Figure 12.7. As an exercise, the novice user is asked to identify the differences between this file and the file Test_1T. The output files corresponding to this simulation (Test_1P.out, Hydrate_Info_1P and Conx_Time_Series_1P) can be found in the directory "TestRuns" in the accompanying CD. 
Review of the output files indicates that the evolution of temperature during dissociation conforms to expectations, exhibiting sharp and rapid declines. A very important observation is the much faster response of the hydrate to depressurization than to thermal stimulation. This difference in response was expected because of the very significant velocities at which the pressure and the temperature fronts propagate. The inevitable conclusion from these results (discussed more fully in Section 12.6) is that depressurization is a much more efficient hydrate dissociation process than thermal stimulation. 
"Test_1P": Equilibrium hydrate dissociation/Depressurization TOUGH+ MEMORY ALLOCATION

HYDRATE-EQUILIBRIUM

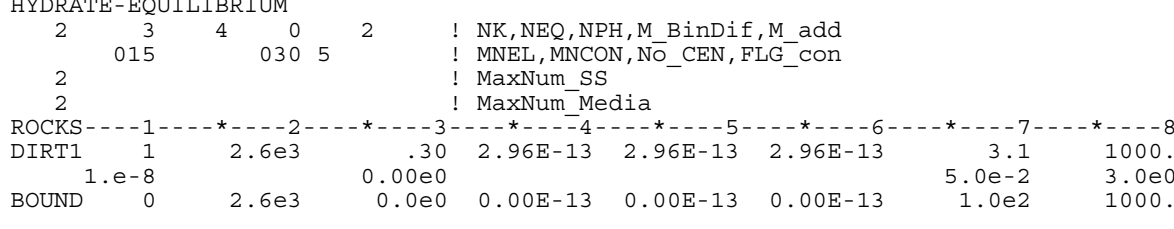

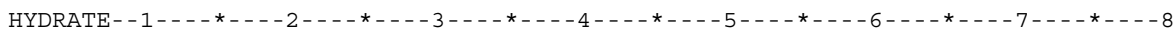

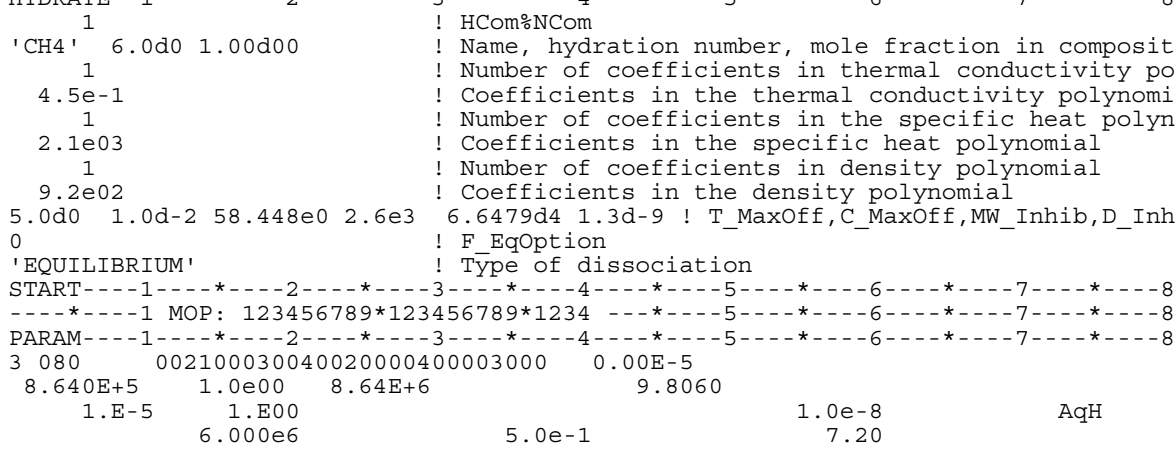

\begin{tabular}{|c|c|c|}
\hline \multicolumn{3}{|l|}{ ELEME } \\
\hline A00 1 & $10.1000 \mathrm{E}+000.4000 \mathrm{E}+00$ & $0.5100 \mathrm{E}-01-.5000 \mathrm{E}+00-.5000 \mathrm{E}+00$ \\
\hline A 002 & $10.1000 \mathrm{E}+000.4000 \mathrm{E}+00$ & $0.1510 \mathrm{E}+00-.5000 \mathrm{E}+00-.5000 \mathrm{E}+00$ \\
\hline A00 3 & $10.1000 \mathrm{E}+000.4000 \mathrm{E}+00$ & $0.2510 \mathrm{E}+00-.5000 \mathrm{E}+00-.5000 \mathrm{E}+00$ \\
\hline A00 4 & $10.1000 \mathrm{E}+000.4000 \mathrm{E}+00$ & $0.3510 \mathrm{E}+00-.5000 \mathrm{E}+00-.5000 \mathrm{E}+00$ \\
\hline A 005 & $10.1000 \mathrm{E}+000.4000 \mathrm{E}+00$ & $0.4510 \mathrm{E}+00-.5000 \mathrm{E}+00-.5000 \mathrm{E}+00$ \\
\hline A00 6 & $10.1000 \mathrm{E}+000.4000 \mathrm{E}+00$ & $0.5510 \mathrm{E}+00-.5000 \mathrm{E}+00-.5000 \mathrm{E}+00$ \\
\hline A00 7 & $10.1000 \mathrm{E}+000.4000 \mathrm{E}+00$ & $0.6510 \mathrm{E}+00-.5000 \mathrm{E}+00-.5000 \mathrm{E}+00$ \\
\hline A00 8 & $10.1000 \mathrm{E}+000.4000 \mathrm{E}+00$ & $0.7510 \mathrm{E}+00-.5000 \mathrm{E}+00-.5000 \mathrm{E}+00$ \\
\hline A00 9 & $10.1000 \mathrm{E}+000.4000 \mathrm{E}+00$ & $0.8510 \mathrm{E}+00-.5000 \mathrm{E}+00-.5000 \mathrm{E}+00$ \\
\hline A 0010 & $10.1000 \mathrm{E}+000.4000 \mathrm{E}+00$ & $0.9510 \mathrm{E}+00-.5000 \mathrm{E}+00-.5000 \mathrm{E}+00$ \\
\hline & & \\
\hline & $-020.4000 \mathrm{E}-02$ & $5000 \mathrm{E}-03-.5000 \mathrm{E}+00-.5000$ \\
\hline
\end{tabular}

A00 0

$10.5000 \mathrm{E}-030.5000 \mathrm{E}-010.1000 \mathrm{E}+01$

$10.5000 \mathrm{E}-010.5000 \mathrm{E}-010.1000 \mathrm{E}+01$

$10.5000 \mathrm{E}-010.5000 \mathrm{E}-010.1000 \mathrm{E}+01$

$10.5000 \mathrm{E}-010.5000 \mathrm{E}-010.1000 \mathrm{E}+01$

$10.5000 \mathrm{E}-010.5000 \mathrm{E}-010.1000 \mathrm{E}+01$

$10.5000 \mathrm{E}-010.5000 \mathrm{E}-010.1000 \mathrm{E}+01$

10.5000E-010.5000E-010.1000E+01

$10.5000 \mathrm{E}-010.5000 \mathrm{E}-010.1000 \mathrm{E}+01$

$1000 \mathrm{E}+01$

$10.5000 \mathrm{E}-010.5000 \mathrm{E}-010.1000 \mathrm{E}+01$

$10.5000 \mathrm{E}-010.5000 \mathrm{E}-030.1000 \mathrm{E}+01$

A 0010 A 0011

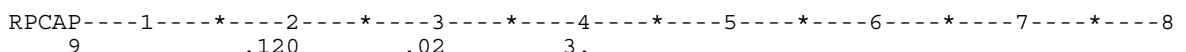
7
$45000 \quad 1.10 \mathrm{e}-1 \quad 8.0 \mathrm{E}-05$

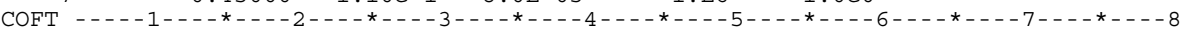

$\mathrm{A} 00 \quad 0 \mathrm{~A} 00 \quad 1$

GENER

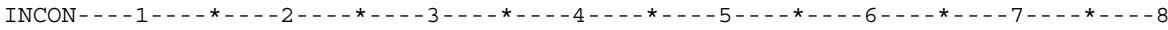

$\mathrm{A} 000 \quad 0.30000000 \mathrm{E}+00 \mathrm{Aqu}$

$2.700 \mathrm{er} \quad 1.00$

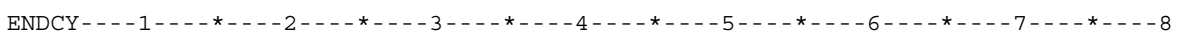

Figure 12.7. Input file for example problem Test_1Pk (in Section 12.4) involving depressurization, equilibrium dissociation, and no inhibitor.

\subsection{Problem Test_1Pk: Depressurization, Kinetic Dissociation, No Inhibitor}


Hydrate dissociation is treated as a kinetic reaction in Test_1Pk. In all other respects, the problem is identical to that in Test_1P, as inspection of the input file in the directory "TestRuns" readily reveals. The output files corresponding to this simulation(Test_1Pk.out, Hydrate_Info_1Pk, Conx_Time_Series_1Pk) can also be found in the same directory.

Review of the output files reveals a dissociation patterns with pressure, temperature and saturation distributions entirely analogous to those for the equilibrium dissociation problem. As in the case of thermal stimulation (see Section 12.3), kinetic hydrate dissociation results in substantially longer execution times and slower time advance in the simulations. This is caused by the more computationally intensive system, the larger (because of the additional degree of freedom) and more difficult-to-solve matrix, and by the parameters of kinetic dissociation that impose time step limitations. These results are more thoroughly discussed in Section 12.6.

\subsection{Problem Test_1P_Ice: Thermal Stimulation, Kinetic Dissociation, No Inhibitor, Ice Evolution}

The only difference between this problem and that in Test_1P is in the boundary

pressure at $x=0$. By setting $P_{b}=5 \times 10^{5} \mathrm{~Pa}$, the boundary is kept at a pressure significantly lower than that at the quadruple point of $\mathrm{CH}_{4}$-hydrate. The lower pressure is expected to lead to faster and larger $\mathrm{CH}_{4}$ release because of the significantly larger pressure differential that drives dissociation, as well as to the emergence of ice as the system tends to the boundary pressure. Of particular interest are the consequences of ice 
on the relative permeability of gas. Unless properly managed, the emergence of ice can significantly reduce the pore space available to gas flow because ice has a lower density than the liquid water it replaces, and can adversely affect flow and gas production.

All the input and output files corresponding to the problem Test_1P_ice can be found in the directory "TestRuns", where the standard TOUGH + output Test_1P_ice.out (showing the changes in the phase regimes during the simulation) is also located. From its original state on the $\mathrm{L}+\mathrm{H}$ phase regime, the rapid depressurization induces $\mathrm{CH}_{4}$-hydrate dissociation, gas emergence, and the appearance of the $\mathrm{L}+\mathrm{G}+\mathrm{H}$ regime. As depressurization and dissociation continue, ice evolves as the system reaches the quadruple point, where it remains at a constant pressure and temperature until the hydrate is exhausted. Afterwards, the $\mathrm{A}+\mathrm{I}+\mathrm{G}$ phase regime appears, during which pressure changes but the temperature remains constant because of the liquid water-ice coexistence, and equal to that of the triple point of water. Because the $x=0$ boundary is kept at a temperature above freezing, ice melts eventually, and an aqueous and gas system remains.

The results of the illustrative problems in problems Test_1P, Test_1Pk and Test_1P_ice are compared in Figures 12.8 to 12.11. It should be noted that relatively coarse discretization has an effect on the results, but appears less pronounced than in the case of thermal stimulation.

Figure 12.8 shows the cumulative rates of $\mathrm{CH}_{4}$ release from the dissociation of hydrate. Unlike the case of thermal dissociation, both kinetic and heat transfer limitations can affect dissociation in depressurization-induced gas release from hydrates. This is demonstrated in Figure 12.8, which shows very different initial rate patterns for the 
equilibrium and the kinetic problems. After the initial a burst of very rapid gas release at the very beginning of the population (corresponding to the maximum deviation from equilibrium), the rate for kinetic dissociation at early times is substantially lower than that for equilibrium dissociation. The relationship is inversed at later times, and eventually the $\mathrm{CH}_{4}$ release rates for kinetic and equilibrium dissociation become about the same as the entire system approaches equilibrium. As expected, the release rate of $\mathrm{CH}_{4}$ from hydrate dissociation is substantially higher in problem Test_1P_ice because of the larger pressure differential. The higher dissociation rate leads to exhaustion of the hydrate at a much earlier time than in the other two problems, leading to zero release and denoted by the vertical segment of the curve at about $t=0.01$ days.

In Figure 12.9, the cumulative gas volumes released from the hydrate in problems Test_1P and Test_1Pk are different at very early times, but become similar later. The cumulative gas rate for problem Test_1P_ice offers stark differences, and shows much larger volumes and exhaustion of the hydrate (marked by the flat part of the curve). The obvious implication is that, as long as potentially adverse relative permeability effects can be avoided, enhanced depressurization provides faster rates and early gas recovery. Compared to the Figures 12.3 and 12.4, Figures 12.5 and 12.6 convincingly demonstrate the superiority and efficiency of depressurization as a method of gas production from gas hydrates. Note that Figures $\mathbf{1 2 . 8}$ and $\mathbf{1 2 . 9}$ were plotted using the data from the output files Hydrate_Info_xxx.

In these problems, gas is produced as it escapes into the low-pressure boundary at $x=$ 0. Figures 12.10 and 12.11 (based on data from the Conx_Time_Series_Xxx output files) show the volumetric production rate of $\mathrm{CH}_{4}$ and the cumulative $\mathrm{CH}_{4}$ production. 
Because of the $\mathrm{L}+\mathrm{H}$ initial regime, all the $\mathrm{CH}_{4}$ originates from the dissociation of the hydrate. Figure 12.10 shows that the $\mathrm{CH}_{4}$ production rate (i.e., the fluxes across the $x=$ 0 boundary) for kinetic dissociation lags behind that for equilibrium dissociation at a very early time, then exceeds it, and finally the two become roughly equal at later times. The production rate for the Test_1P_ice problem is significantly larger, and becomes zero at a very early time because no hydrate is left in the system (see the Conx_Time_Series_1P_ice file in Appendix A3).

In Figure 12.11, the total gas production (measured as the $\mathrm{CH}_{4}$ volume that crosses the $x=0$ boundary) from the kinetic dissociation problem remains consistently lower than that for the equilibrium dissociation case, and the maximum deviation occurs at a very early time. Similarly to the pattern observed in Figure 12.9, gas production in the Test_1P_ice problem is significantly larger and leads to very early disappearance of the hydrate. A comparison of Figures $\mathbf{1 2 . 1 0}$ and $\mathbf{1 2 . 1 1}$ to the analogous ones for thermal stimulation (Figures 12.5 and 12.6) further confirm the superiority of depressurization as a dissociation strategy. 


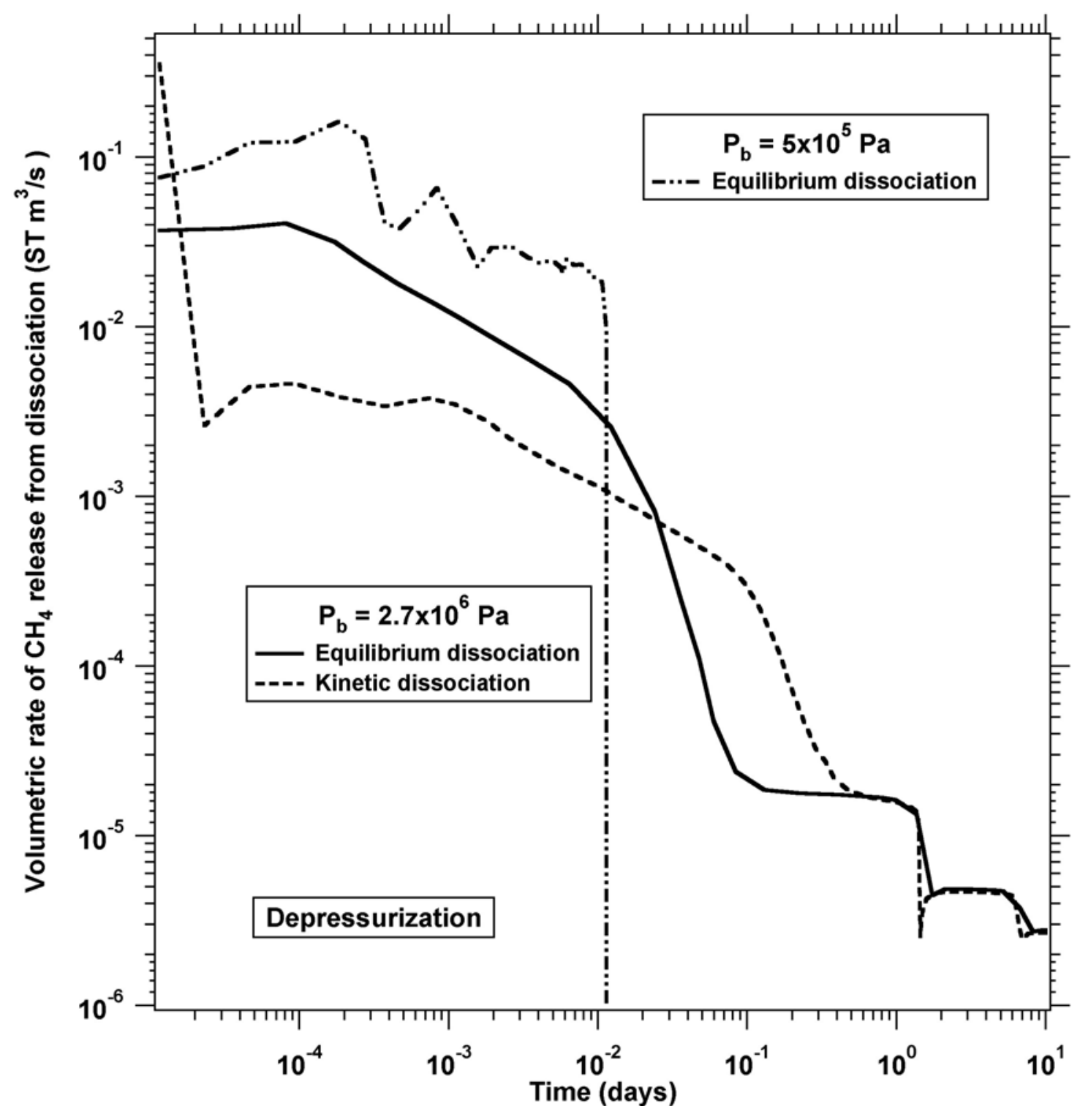

Figure 12.8. Comparison of the volumetric rates of $\mathrm{CH}_{4}$ release from depressurization-induced hydrate dissociation in problems Test_1P, Test_1Pk and Test_1P_ice. 


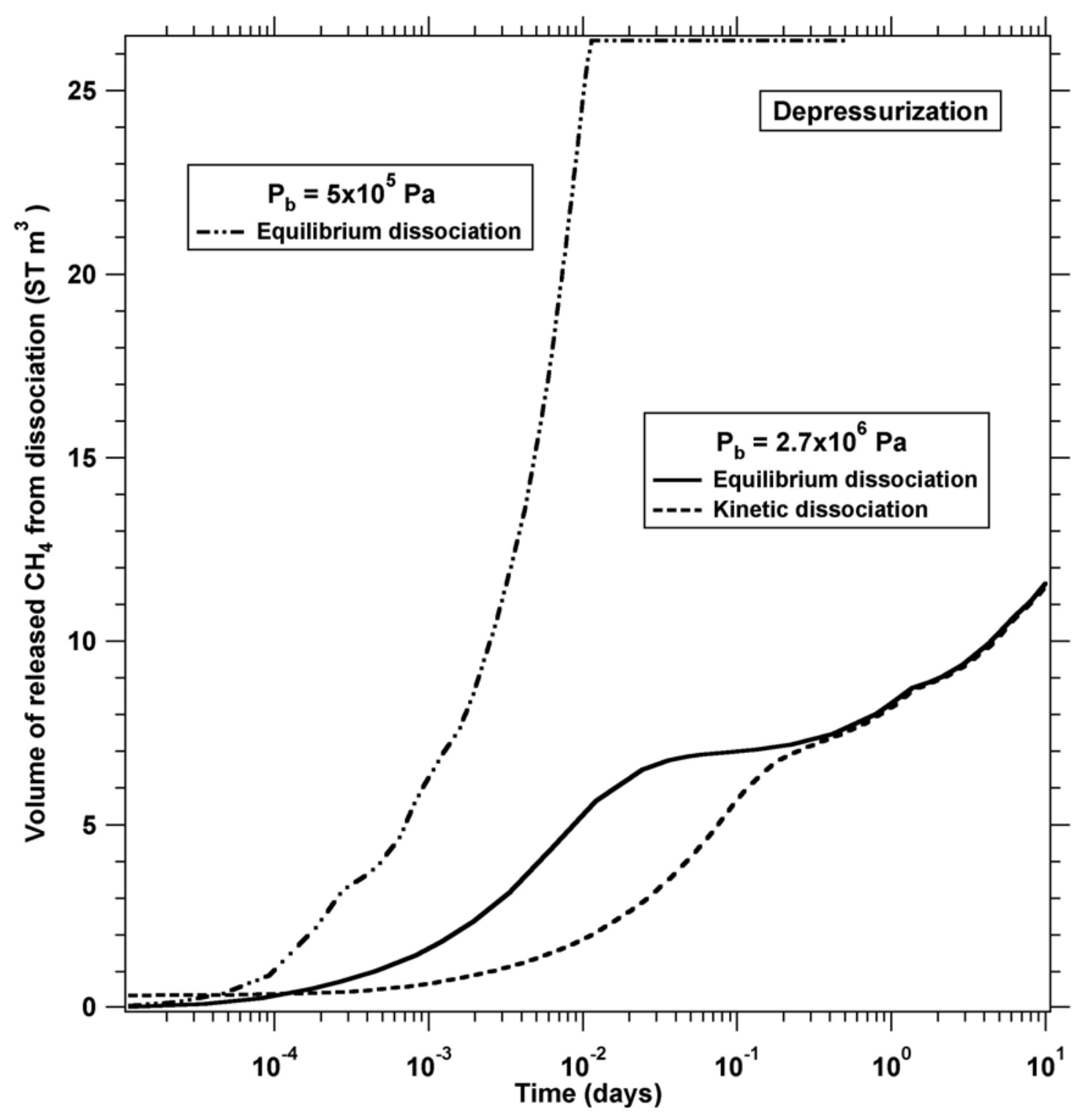

Figure 12.9. Comparison of the cumulative volumes of $\mathrm{CH}_{4}$ released from depressurizationinduced hydrate dissociation in problems Test_1P, Test_1Pk and Test_1P_ice. 


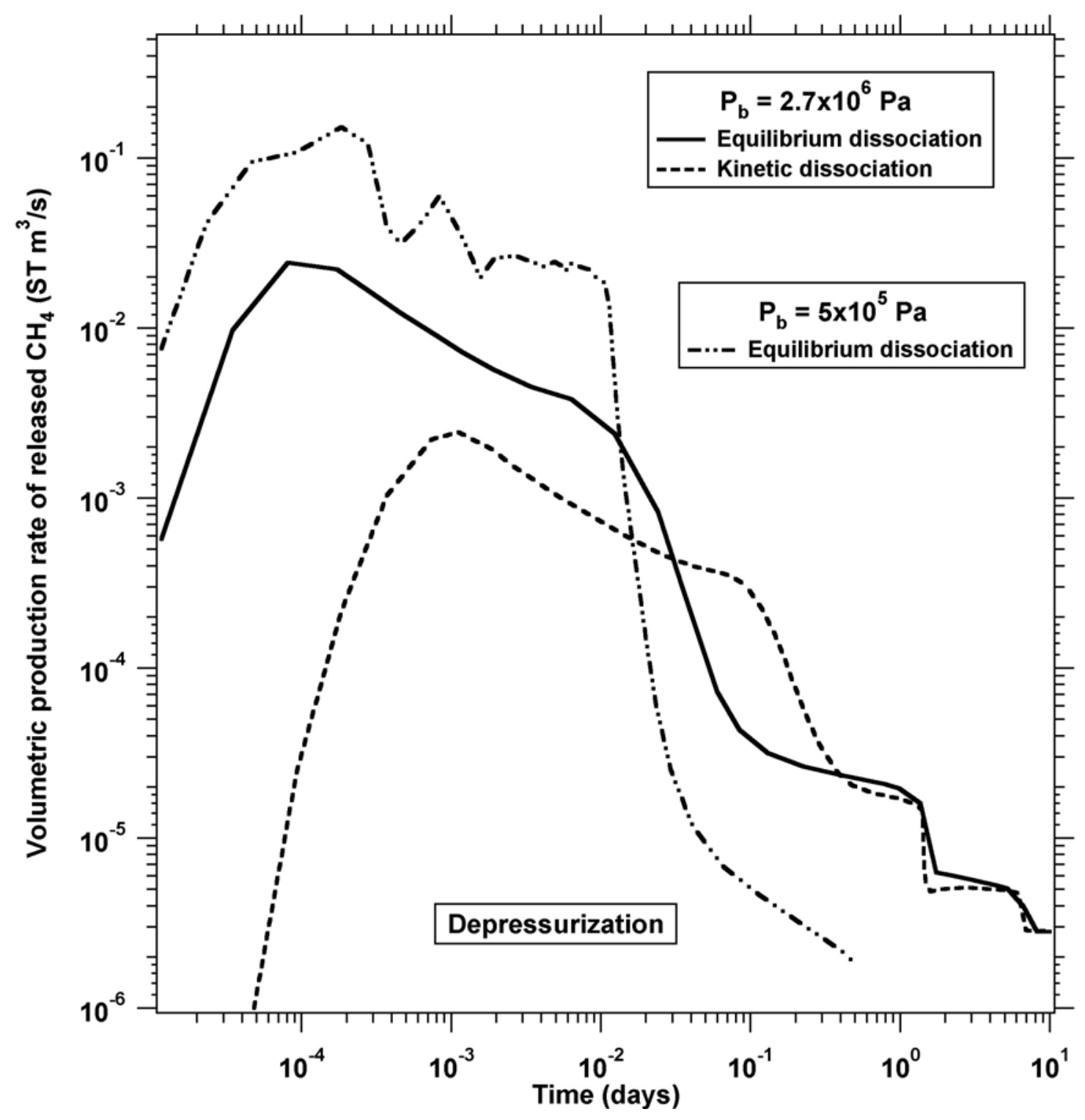

Figure 12.10. Comparison of the volumetric production rates of $\mathrm{CH}_{4}$ (measured as the flux crossing the $x=0$ boundary) in problems Test_1P, Test_1Pk and Test_1P_ice. 


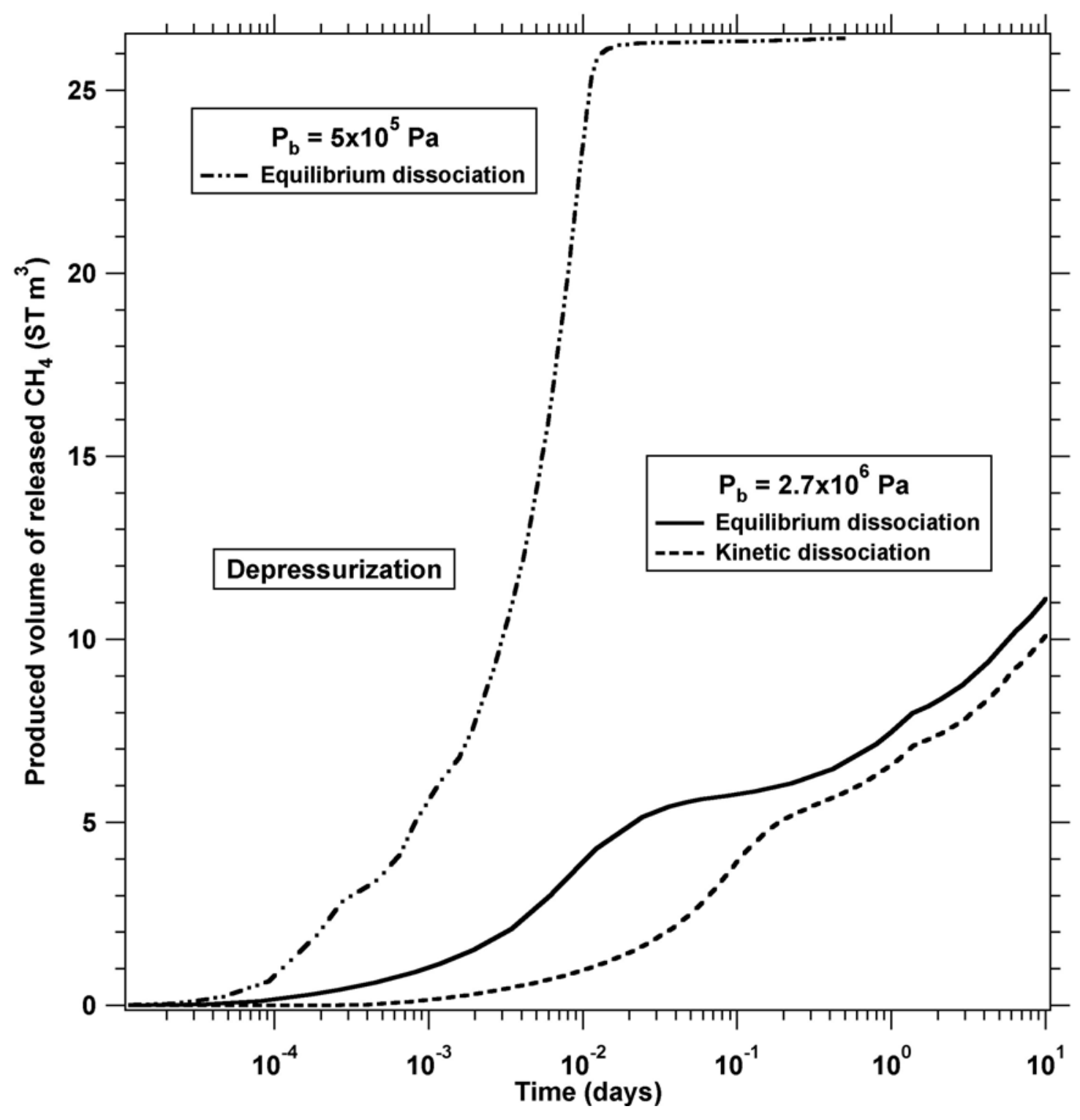

Figure 12.11. Comparison of the total production volumes of $\mathrm{CH}_{4}$ (measured as the total volumes crossing the $x=0$ boundary) in problems Test_1P, Test_1Pk and Test_1P_ice. 


\subsection{Problem Test_1TbS and Test_1TbSk: Hydrate Formation, Equilibrium and Kinetic Hydration reaction, Inhibitor}

The porous medium in 1-D columns in problems Test_1TbS and Test_1TbSk has the same properties as those reported in problem Test_1P (see Section 12.2), but the systems have different initial and boundary conditions. Because the pressure $P=$ $4 \times 10^{6} \mathrm{~Pa}$ and temperature $T=8.5{ }^{\circ} \mathrm{C}$ are outside the stability zone of hydrate, the pore space is filled by a two-phase system involving a gas and an aqueous phase. The aqueous phase is a $\mathrm{NaCl}$ solution, in which the initial mass fraction of the salt is $X=10^{-3}$. The column is attached to a constant pressure boundary at $x=L_{\max }$ that can provide fluids to the active domain. The $x=0$ boundary is impermeable, thermally conductive, and at a constant temperature of $T_{b}=1.2{ }^{\circ} \mathrm{C}$. Because of conduction, the temperature in the column is expected to drop below the hydration temperature at the prevailing pressure, leading to the formation of hydrate. The temperature decline is somewhat buffered by the exothermic reaction of hydrate formation, and the formation process is affected by the presence of an inhibitor. In addition to the original equilibrium problem, a second case was investigated, in which the initial temperature was higher, with $T=4.5{ }^{\circ} \mathrm{C}$. The lower initial $T$ is expected to lead to earlier and faster hydrate formation.

An equilibrium hydrate reaction is assumed in problem Test_1TbS, while a kinetic hydrate reaction is assumed in Test_1TbSk. The input files and all the corresponding output files are available in the directory "TestRuns". The outputs corresponding to the lower initial $T$ have the identifier ' 1 ThS2'. A review of the standard TOUGH+ output files clearly shows the evolution of hydrate. Of particular interest is the increase 
in the inhibitor concentration as hydrate is formed. This is consistent with expectations because inhibitors cannot be partitioned into the solid hydrates. As in all previous kinetic cases, the kinetic treatment of the hydration equation results in longer execution times and slower advancement of the simulation time.

Figure 12.12 shows the cumulative rates of $\mathrm{CH}_{4}$ consumption during hydrate formation. With the exception of results at very early times (when the rate corresponding to hydrate formation is larger because of maximum deviation from equilibrium), the $\mathrm{CH}_{4}$ consumption rate for $T=8.5^{\circ} \mathrm{C}$ are practically identical for both kinetic and equilibrium hydrate reaction. This was expected because the heat removal occurs through conduction, which is a very slow process and dominates the reaction. Additionally, the onset of hydrate evolution occurs at the same time for both cases.

The $\mathrm{CH}_{4}$ consumption rate for the $T=4.5^{\circ} \mathrm{C}$ equilibrium reaction case is significantly larger, and hydrate evolves at an earlier time. These results are reflected in Figure 12.13, which shows the cumulative $\mathrm{CH}_{4}$ consumption during the formation process. The total volume of reacted $\mathrm{CH}_{4}$ is the same for both the $T=8.5{ }^{\circ} \mathrm{C}$ kinetic and equilibrium reaction, while it is much larger for the $T=4.5^{\circ} \mathrm{C}$ equilibrium reaction (i.e., the amount consumed is much lower). 


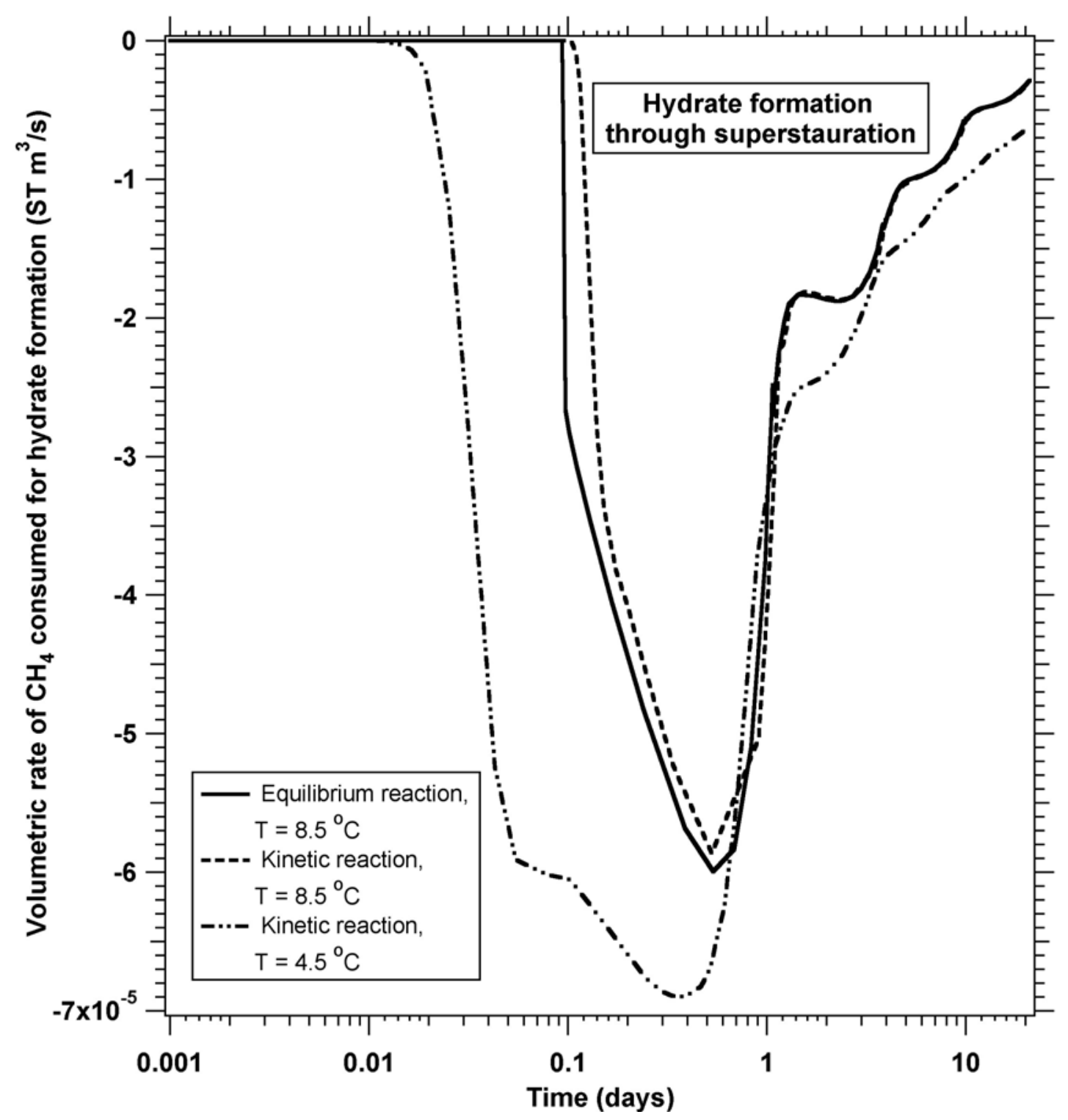

Figure 12.12. Comparison of the volumetric rates of $\mathrm{CH}_{4}$ consumption during hydrate formation in problems Test_1TbS, Test_1TbSk and Test_1TbS2. 


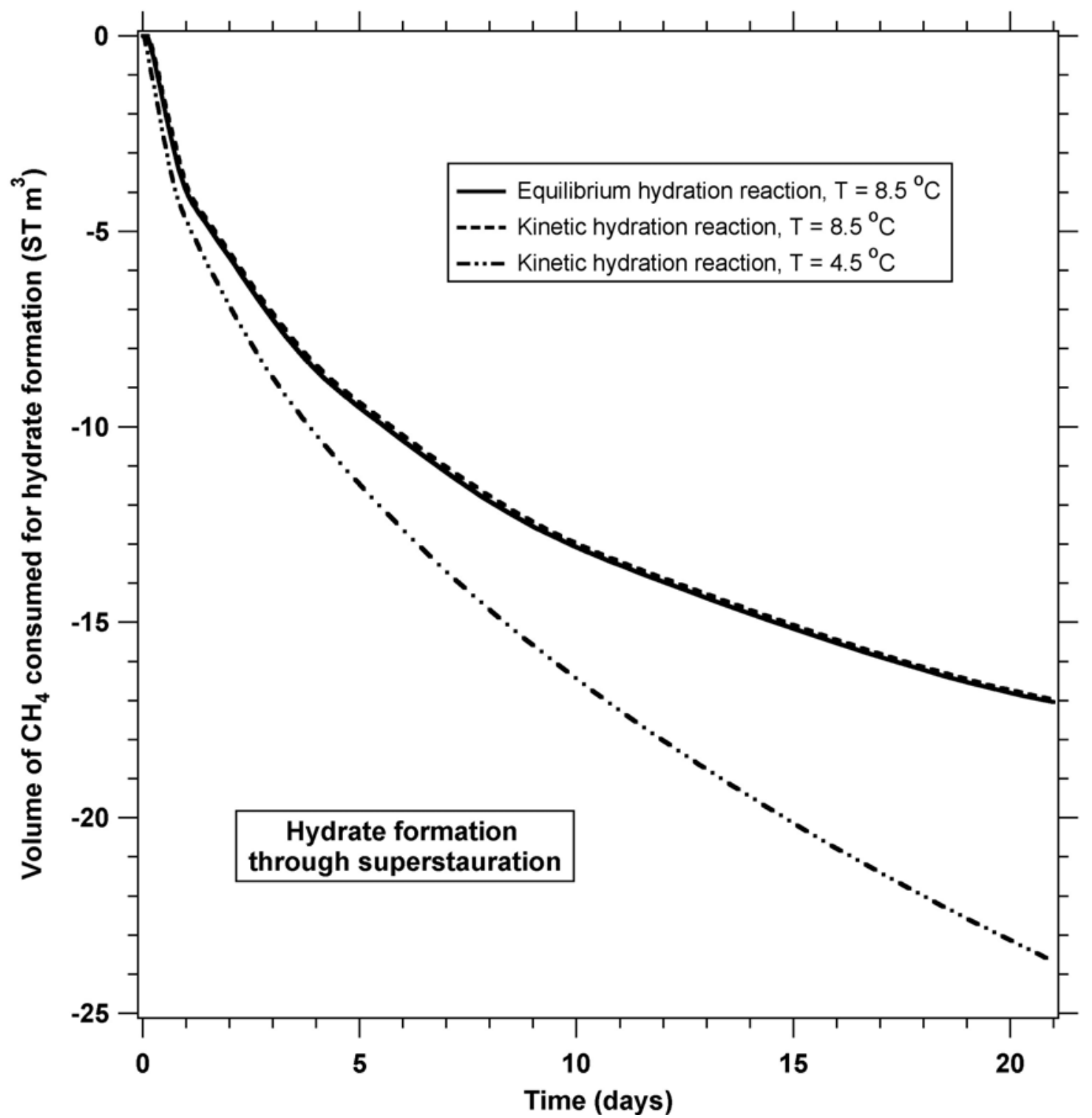

Figure 12.13. Comparison of the cumulative volumes of $\mathrm{CH}_{4}$ consumed during hydrate formation in problems Test_1TbS, Test_1TbSk and Test_1TbS2. 


\subsection{Problem Test_2Qp: Equilibrium Hydrate Dissociation, Depressurization, Radial Grid - Single Well}

Problem Test_2Qp represents a more realistic scenario of gas production from a Class 3 (Moridis and Collett, 2004) hydrate deposit in which water and hydrate are initially at equilibrium. In the deposit, $P=9.8 \times 10^{6} \mathrm{~Pa}, T=12.5{ }^{\circ} \mathrm{C}$, and the initial hydrate saturation is $S_{H}=0.5$. Such conditions can be encountered at the bottom of the stability zone in permafrost accumulations (Moridis et al., 2003). The input file and all the corresponding output files are available in the directory "TestRuns".

This problem involves production from a 10-m thick cylindrical reservoir that extends to $R_{\max }=224 \mathrm{~m}$. The properties of the formation are the same with those in all previous problems (see Section 12.1). A Dirichlet-type (constant conditions equal to the initial conditions) boundary is assumed at $R_{\max }$, i.e., the outer rim of the grid. Fluids are withdrawn at a mass flow rate of $Q=1 \mathrm{~kg} / \mathrm{s}$ through a well at the center of the grid, and are distributed in the production stream according to their mobilities. The fluid withdrawal causes a pressure decline that leads to the depressurization-induced release of $\mathrm{CH}_{4}$.

The grid in the problem is much larger than in the previous examples, but still insufficient to provide the resolution needed for the representation of an infinite-acting system. Because of this, the proximity of the boundary to the well, the near incompressibility of the aqueous phase, and the quantum-type system behavior in equilibrium dissociation, discretization effects are expected to appear in the estimation of the dissociation rates (see earlier discussions). 
The evolution of (a) the cumulative rate of $\mathrm{CH}_{4}$ release from dissociation and (b) the cumulative volume of $\mathrm{CH} 4$ released from dissociation in the repository are shown in Figures 12.14 and 12.15, respectively. As expected, the curve of the cumulative rate of CH4 release exhibits early oscillations attributed to both the rather coarse discretization and the quantum nature of hydrate dissociation. As expected, the release rate increases initially because of advancing depressurization, but begins to decline after a maximum is reached. This decline is caused by the rapid cooling of the hydrate because of advancing dissociation, which affects the rate of dissociation (and the consequent $\mathrm{CH}_{4}$ release) of the remaining hydrate. As discussed earlier, the jagged appearance of the rate curve in Figure 12.14 is caused by the coarse discretization. Conversely, the curve of the cumulative volume of released $\mathrm{CH}_{4}$ is smooth, with the maximum release rate marked by the inflection point at about $t=16$ days. 


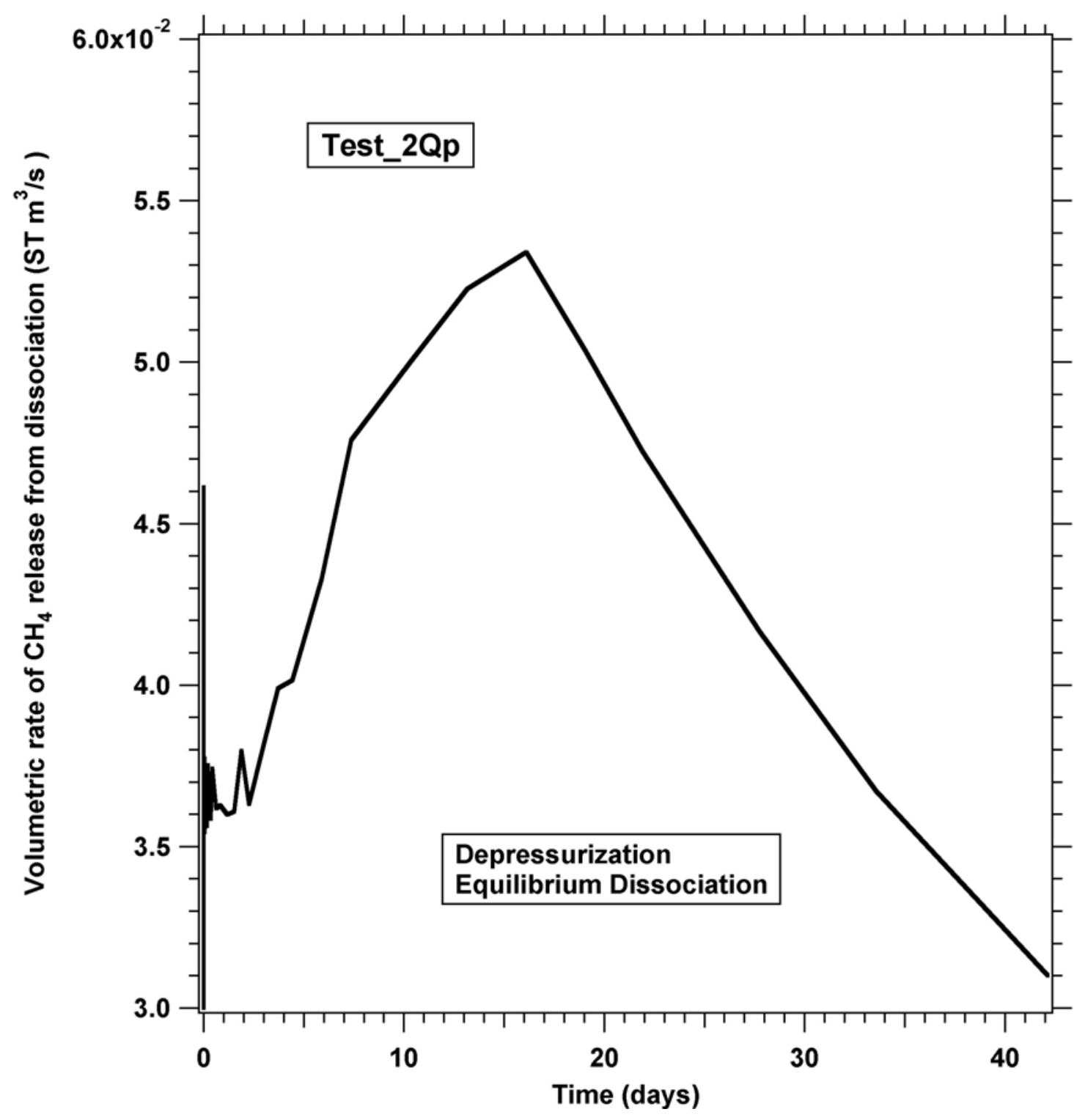

Figure 12.14. Volumetric rate of $\mathrm{CH}_{4}$ release during hydrate dissociation in problem Test_2Qp. 


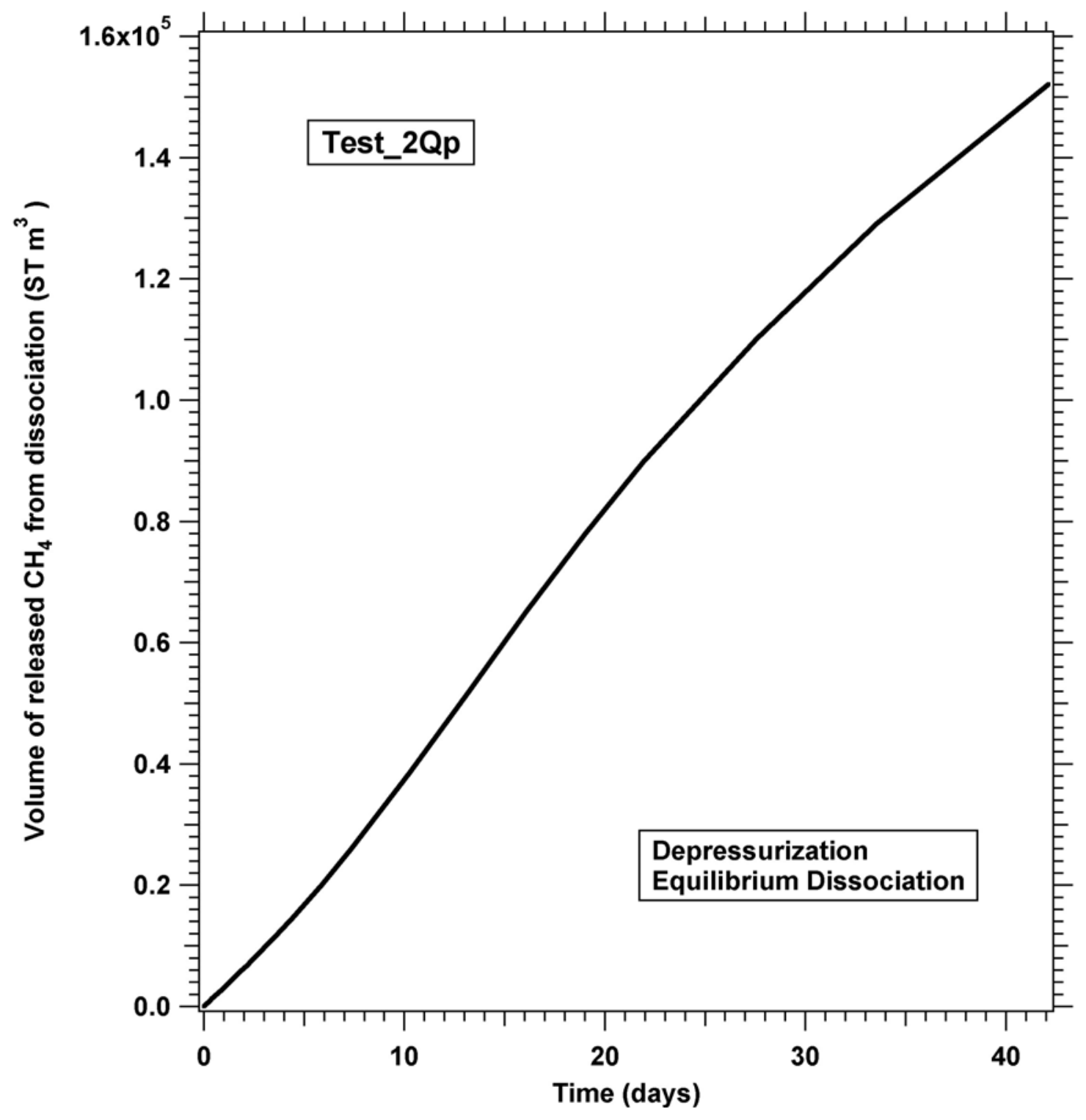

Figure 12.15. Cumulative volume of $\mathrm{CH}_{4}$ released during hydrate dissociation in problem Test_2Qp. 


\subsection{Problem Test_3Qpk: Kinetic Hydrate Dissociation, Depressurization, Radial Grid - Single Well with Wellbore Heating}

Problem Test_3Qpk uses the same grid, and porous medium and boundary conditions as problem Test_2Qp. This deposit, for which $\mathrm{P}=9.51 \times 10^{6} \mathrm{~Pa}$ and $\mathrm{T}=12.5$ ${ }^{\circ} \mathrm{C}$, involves a deposit in which water, gas and hydrate are initially at equilibrium. The initial gas and hydrate saturations are $S_{G}=0.5$ and $S_{H}=0.4$, respectively. A Dirichlettype boundary (constant conditions equal to the initial conditions) is assumed at $R_{\max }$, i.e., the outer rim of the grid. Fluids are withdrawn at a mass flow rate of $Q=0.3 \mathrm{~kg} / \mathrm{s}$ through a well at the center of the grid, and are distributed in the production stream according to their mobilities. The fluid withdrawal causes a pressure decline that leads to the depressurization-induced release of $\mathrm{CH}_{4}$. To alleviate potential hydrate buildup problems that can severely restrict flow to the well (possibly because of temperature drop and availability of gas and water in the vicinity of the well), the wellbore is heated at a rate of $Q_{H}=1.2 \mathrm{KW}$. The input file and all the corresponding output files are available in the directory "TestRuns".

The evolution of the rates of (a) cumulative $\mathrm{CH}_{4}$ release in the deposit, and (b) gas production at the well are shown in Figure 12.16. Because of the coexistence of the three phases in the initial system, the curve of the $\mathrm{CH}_{4}$ release rate is smooth. A comparison of these two curves reveals that the contribution of gas from hydrate dissociation to the rate of gas production decreases progressively with time, and becomes minor at the end of the simulation period. The reason for this performance is the continuing cooling of the hydrate as it dissociates, in addition to the proximity of the well 
to the (constant-conditions) boundary and the relatively large permeability, which do not allow efficient depressurization of the system. Consequently, the quasi steady-state condition that is reached rather early is not conducive to increased gas production because the system adjusts to a new state of equilibrium. The novice user is encouraged to investigate the system behavior if the Dirichlet-type boundary is replaced by a no-flow Neuman boundary.

The cumulative volumes of (a) $\mathrm{CH} 4$ released from dissociation in the repository and (b) produced from the wells are shown in Figure 12.17. The declining contribution of $\mathrm{CH}_{4}$ from dissociation is reflected in the practically flat portion of the released $\mathrm{CH}_{4}$ curve. 


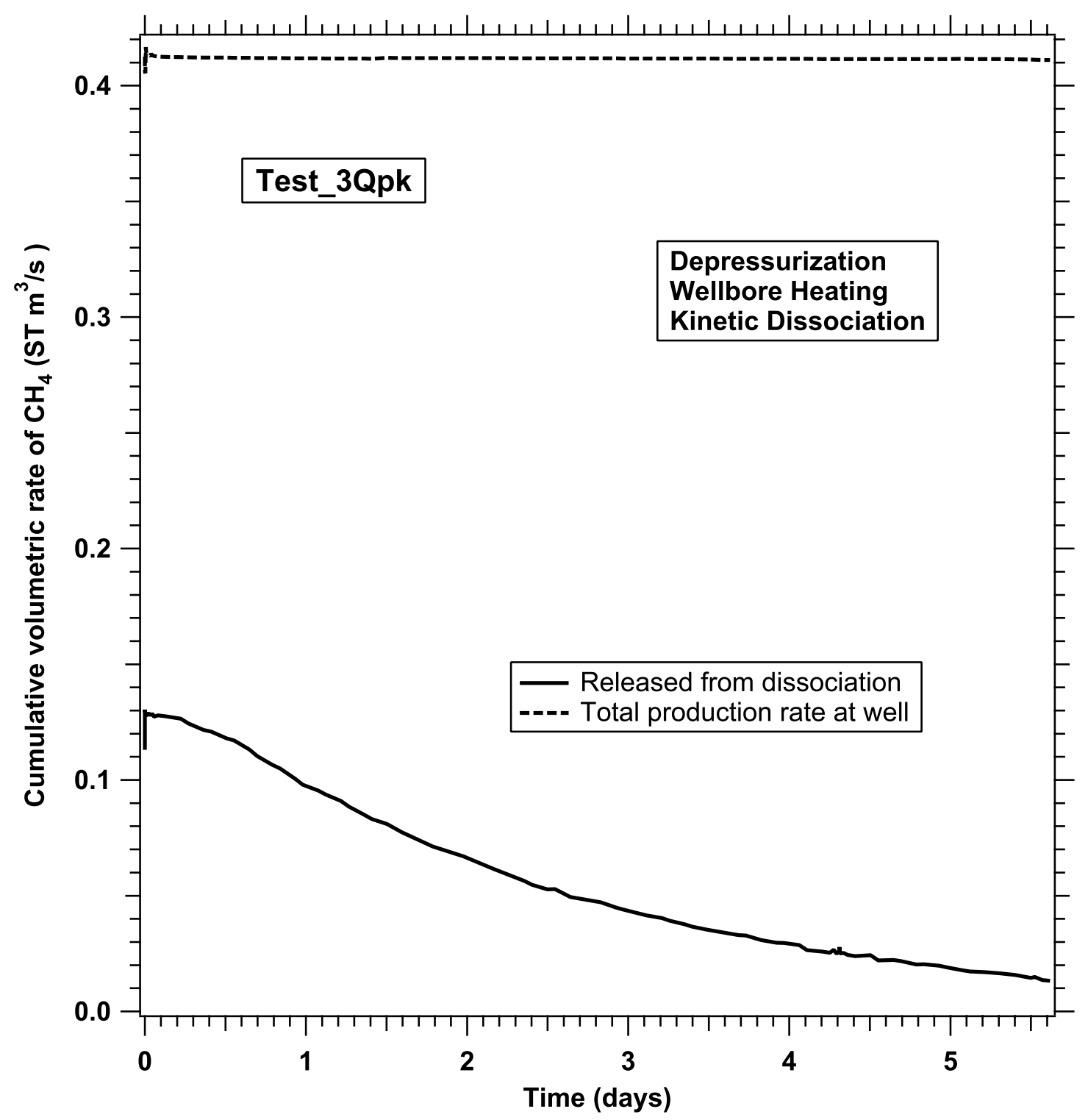

Figure 12.16. Volumetric rates of $\mathrm{CH}_{4}$ (a) release in the reservoir during hydrate dissociation and (b) production from the well in problem Test_3Qpk. 


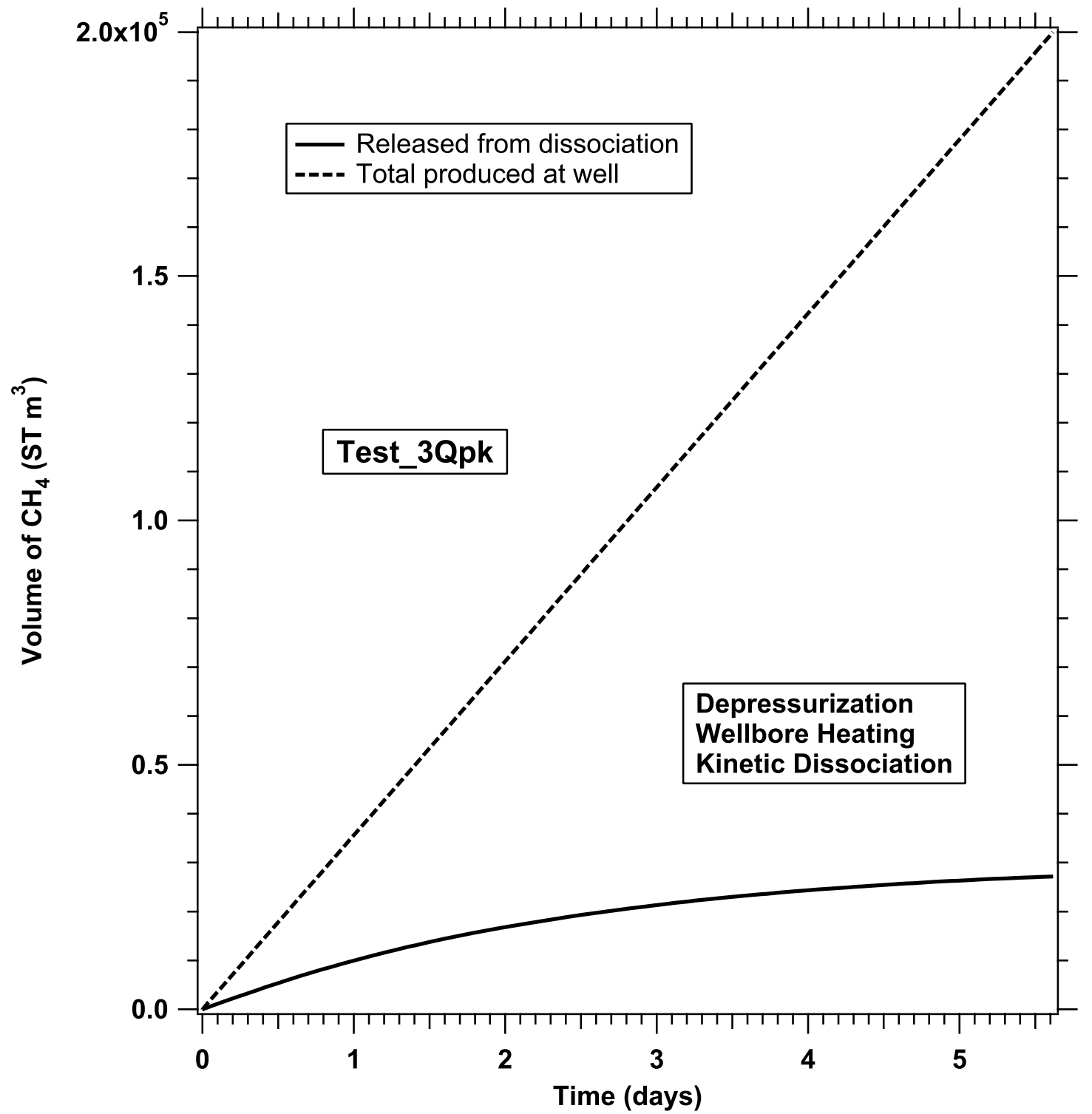

Figure 12.17. Cumulative volumes of $\mathrm{CH}_{4}$ (a) released in the reservoir during hydrate dissociation and (b) produced from the well in problem Test_3Qpk. 


\subsection{Problem Test_2D: Equilibrium Hydrate Dissociation, 2-D Areal System}

Problem Test_2D describes gas production from a realistic areal 2-D hydratebearing formation that has the same porous medium properties as all the previous examples. The simulation domain consists of a square system with a side of $50 \mathrm{~m}$ and a formation thickness of $10 \mathrm{~m}$. The domain is subdivided in 50 x $50=2500$ cells in $(\mathrm{x}, \mathrm{y})$, with the well located in the cell at $(x, y)=(0.5 \mathrm{~m}, 0.5 \mathrm{~m})$. This is considered a part of a large-scale production system that involves a deposit of considerable areal extent and a large number of producing wells. Under these conditions, the simulation domain represents one quarter of the basic production stencil, and its outer boundaries are noflow Neuman-type boundaries because of symmetry. The 2-D geometry of the system automatically implies no flow in the third direction, i.e., the system is treated as insulated along its top and bottom boundaries. While this is not strictly true in porous media, such heat transfer through impermeable boundaries can only occur through conduction, which is a very slow process and is not expected to appreciably affect the results. Additionally, by ignoring heat contribution from its boundaries, this simulation provides the worst-case scenario of gas production from such a hydrate accumulation.

In this deposit, water, gas and hydrate are initially at equilibrium, and the pressure is the hydration pressure corresponding to $T=12.5^{\circ} \mathrm{C}$. The initial gas, aqueous and hydrate phase saturations are $S_{G}=0.1, S_{A}=0.3$ and $S_{H}=0.6$, respectively. Fluids are withdrawn at a mass flow rate of $Q=0.5 \mathrm{~kg} / \mathrm{s}$ through the production well, and are distributed in the production stream according to their mobilities. The fluid withdrawal causes a pressure decline that leads to the depressurization-induced release of $\mathrm{CH}_{4}$. The production flow 
rate remains constant, and is certain to lead to temperature decline and ice appearance because of the endothermic nature of dissociation. The input file and all the corresponding output files are available in the directory "TestRuns". Because of the computationally intensive nature of this realistic problem, the simulation period was limited to two days. Interested users are encouraged to run this problem (modified per their desires) for longer periods.

Figure 12.18 shows the evolution of the cumulative volume of $\mathrm{CH} 4$ released from dissociation in the repository in the first two days of production. Note that the released volume increases practically linearly with time. In this short period, a daily release of about $10000 \mathrm{ST} \mathrm{m}^{3}$ from the hydrate is observed. This is expected to change at later times, when exhaustion of the 'free' gas and hydrate resources in the reservoir will inevitably lead to a sublinear performance.

The pressure distribution in the reservoir at $\mathrm{t}=2$ days in Figure 12.19 indicates substantial pressure declines over an extended portion of the domain, indicating significant hydrate dissociation (as supported by the results in Figure 12.18). The corresponding temperature distribution in Figure 12.20 shows an extended cool region even at that early time, providing additional indirect evidence of dissociation.

Direct evidence of dissociation is provided by the corresponding hydrate distribution at the same time in Figure 12.21, which clearly indicates severe hydrate dissociation within $5 \mathrm{~m}$ from the well, and measurable dissociation as far as $15 \mathrm{~m}$ away from the well. While these are very positive and encouraging results, they are not indicative of the production potential if there is no information on the relative permeability regime in the vicinity of the wellbore. The production potential of the hydrate accumulation can be 
seriously diminished if the water (released from the hydrate dissociation) or the ice (that can be formed in the course of the endothermic reaction) inhibit the flow of gas toward the well.

The answer to this question is provided by the distributions of the aqueous and gas phase saturations at $t=2$ days in Figures 12.22 and 12.23. While the water saturation increases in the vicinity of the well in the process of dissociation, the gas saturation increases also. Such a desirable regime then becomes a production management and design issue in the design of appropriate production strategies from the hydrate deposit. 


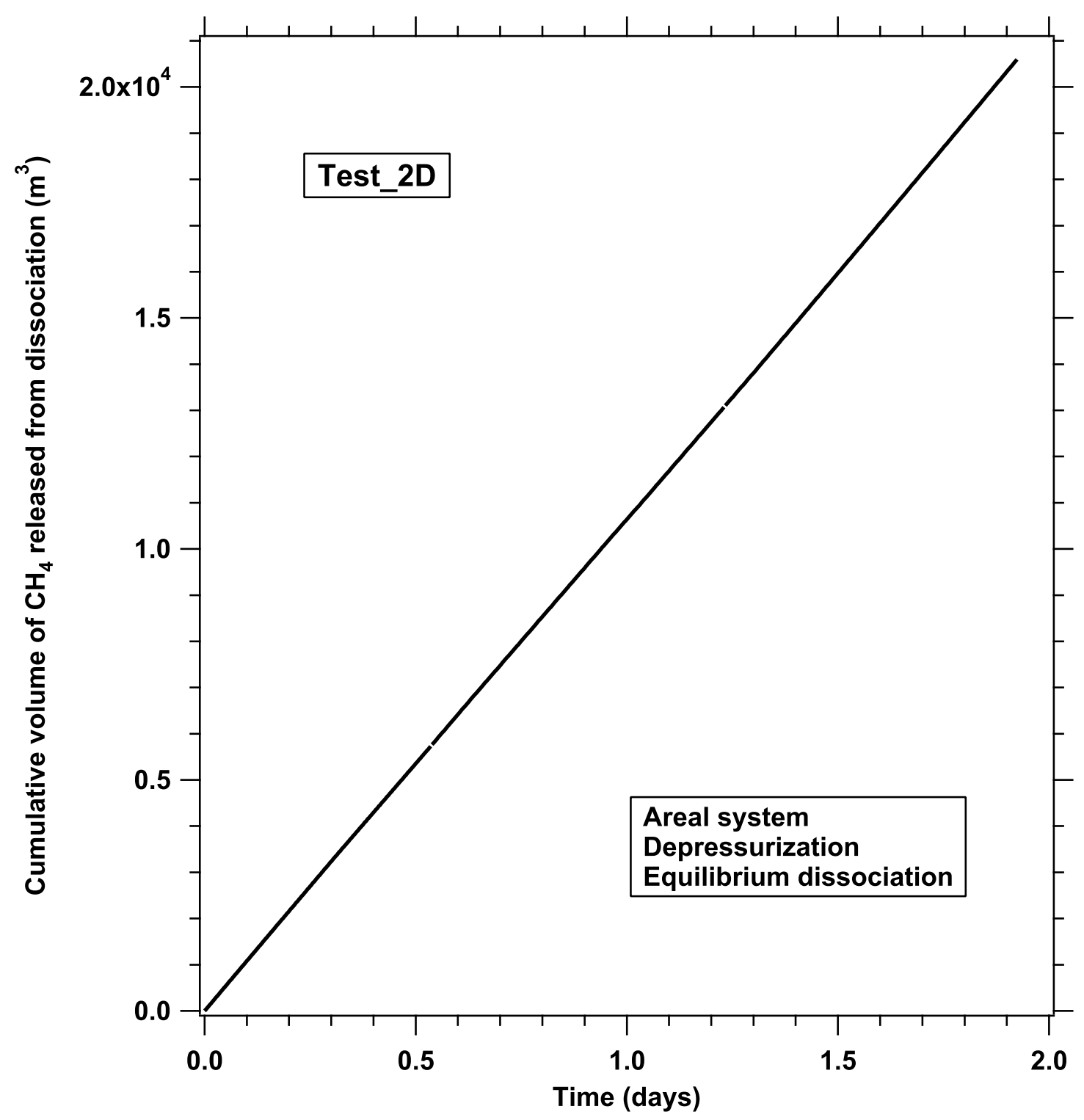

Figure 12.18. Volumetric rates of $\mathrm{CH}_{4}$ release from the hydrate in the reservoir during gas production in problem Test_2D. 


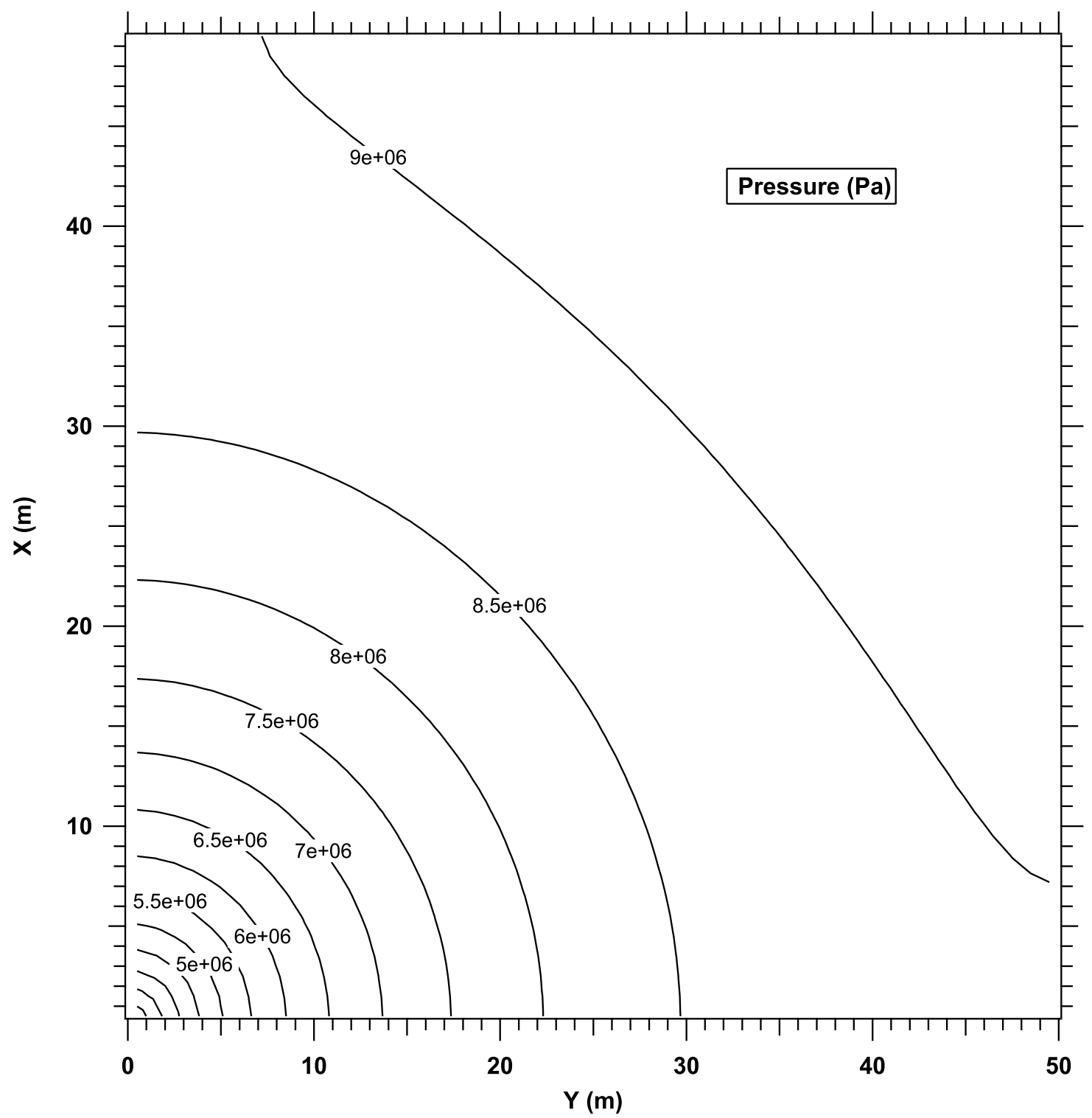

Figure 12.19. Pressure distribution in the reservoir at $t=2$ days in problem Test_2D. 


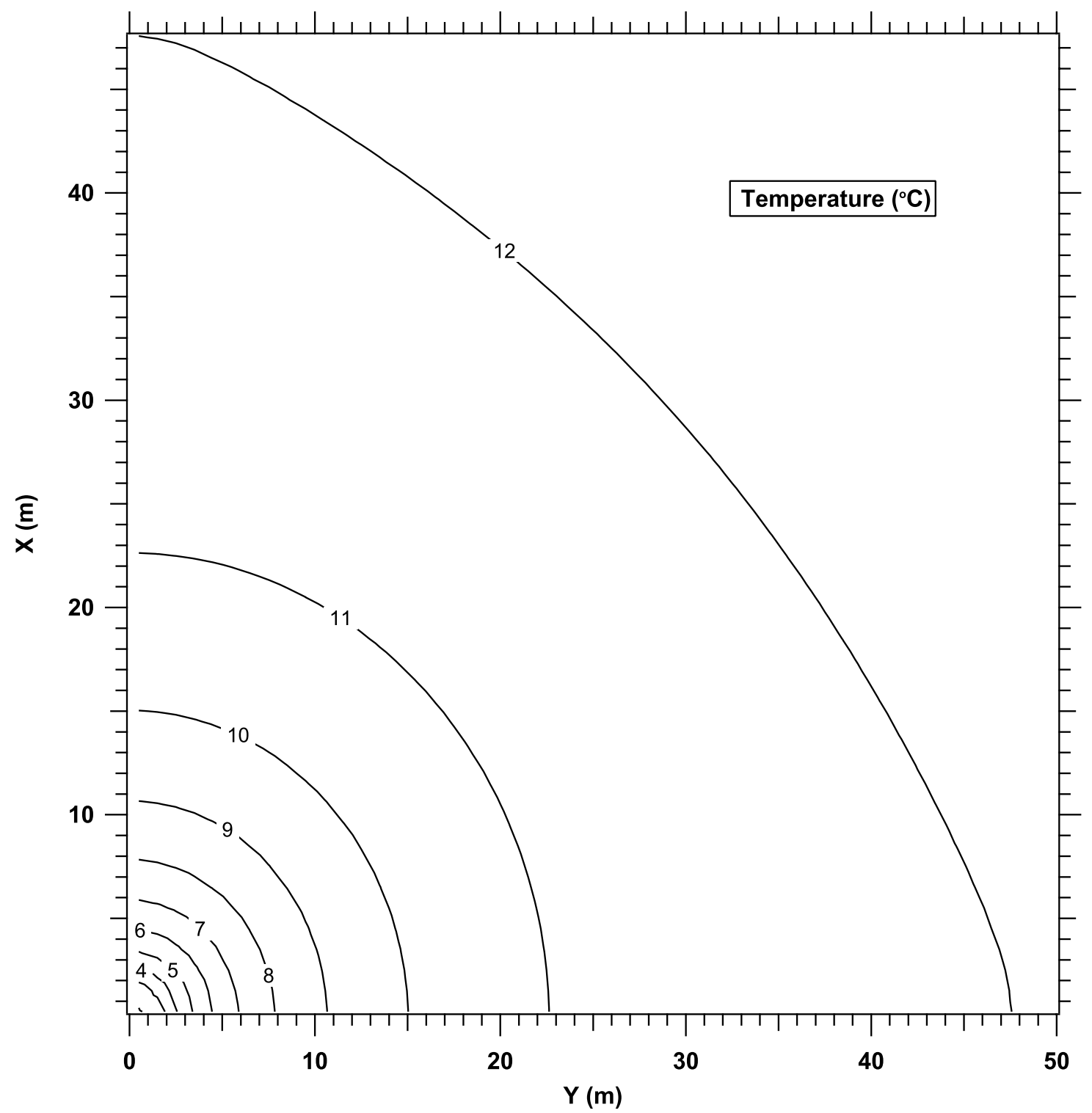

Figure 12.20. Temperature distribution in the reservoir at $t=2$ days in problem Test_2D. 


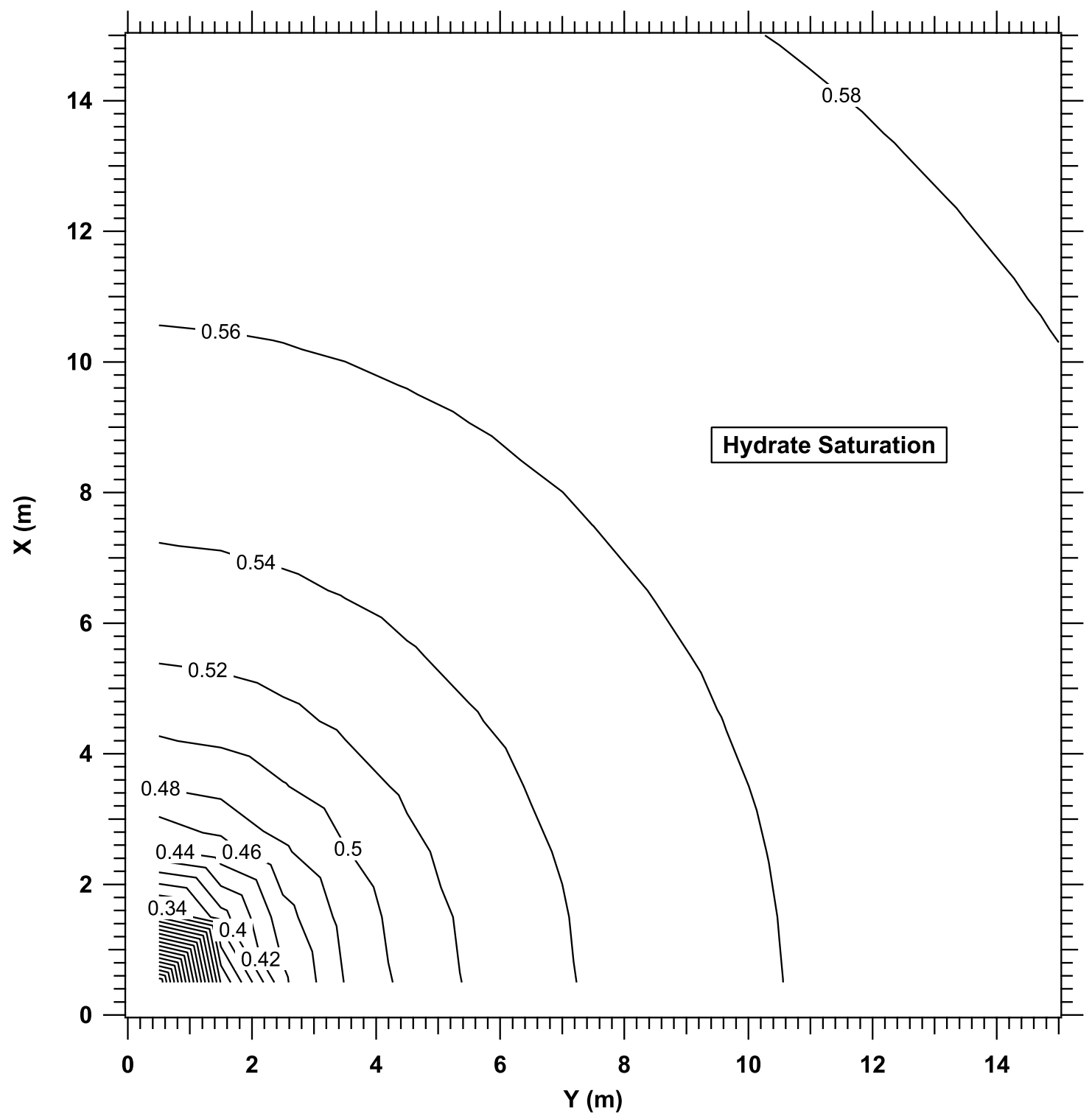

Figure 12.21. Hydrate saturation distribution in the reservoir at $t=2$ days in problem Test_2D. 


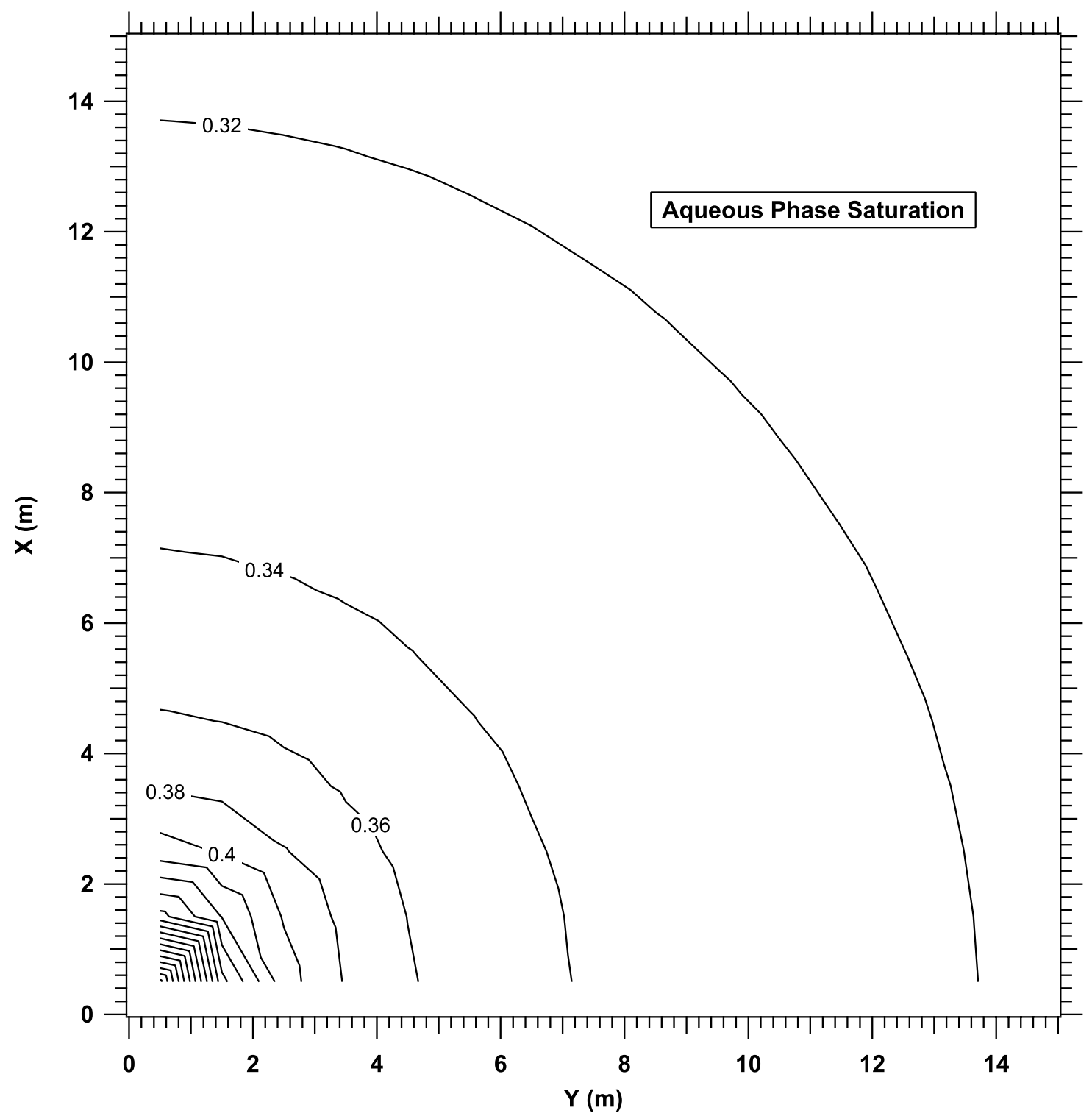

Figure 12.22. Aqueous phase saturation distribution in the reservoir at $t=2$ days in problem Test_2D. 


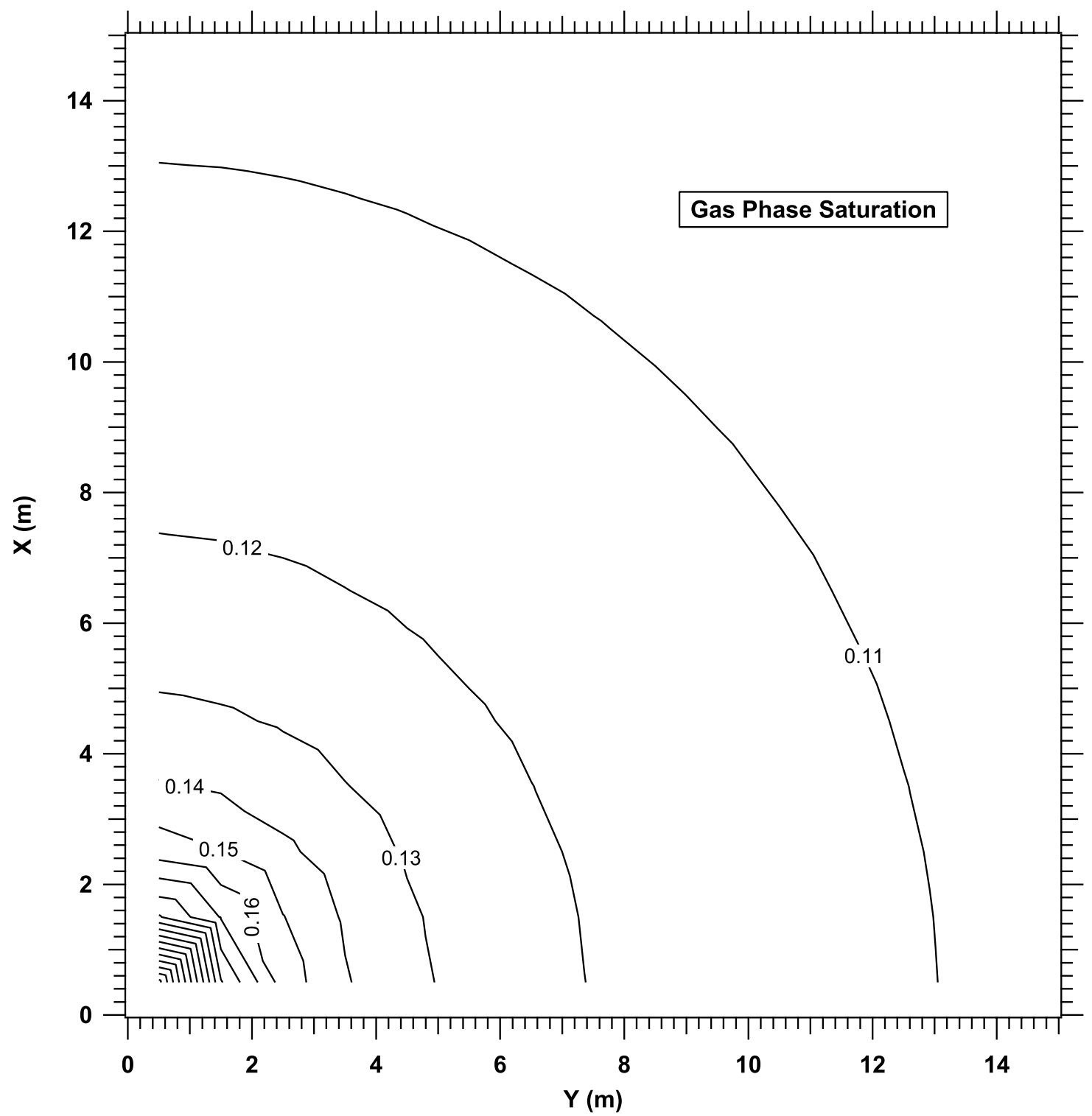

Figure 12.23. Gas saturation distribution in the reservoir at $t=2$ days in problem Test_2D. 


\section{Acknowledgements}

This work was supported by the Assistant Secretary for Fossil Energy, Office of Natural Gas and Petroleum Technology, through the National Energy Technology Laboratory, under the U.S. Department of Energy, Contract No. DE-AC03-76SF00098. 


\section{References}

Atkinson, P.G., R. Celati, R. Corsi and F. Kucuk. Behavior of the Bagnore Steam/CO2 Geothermal Reservoir, Italy, Soc. Pet. Eng. J., 228 - 238, 1980.

Aunzo, Z.P., G. Bjornsson and G.S. Bodvarsson. Wellbore Models GWELL, GWNACL, and HOLA, Lawrence Berkeley National Laboratory Report LBL-31428, Berkeley, CA, October 1991.

Aziz, K. and A. Settari. Petroleum Reservoir Simulation, Elsevier, London and New York, 1979.

Battistelli, A., C. Calore and K. Pruess. The Simulator TOUGH2/EWASG for Modeling Geothermal Reservoirs with Brines and Non-Condensible Gas, Geothermics, Vol. 26, No. 4, pp. 437 - 464, 1997.

Bird, R., W.E. Stewart and E.N. Lightfoot. Transport Phenomena, John Wiley \& Sons, New York, NY, 1960.

Brand, C.W., J.E. Heinemann and K. Aziz. The Grid Orientation Effect in Reservoir Simulation, Paper SPE-21228, presented at Society of Petroleum Engineers Eleventh Symposium on Reservoir Simulation, Anaheim, CA, February 1991.

Cass, A., G.S. Campbell and T.L. Jones. Enhancement of Thermal Water Vapor Diffusion in Soil. Soil Sci. Soc. Am. J., Vol. 48, no. 1, pp. 25 - 32, 1984.

Clarke, M., and Bishnoi, P.R., Determination of activation energy and intrinsic rate constant of methane gas hydrate decomposition; Canadian Journal of Chemical Engineering, v. 79, no.1, p. 143-147, 2001.

Coats, K.H. Geothermal Reservoir Modeling, paper SPE-6892, presented at the 52nd Annual Fall Technical Conference and Exhibition of the SPE, Denver, Colorado, October 1977.

Coats, K.H. and A.B. Ramesh. Effects of Grid Type and Difference Scheme on Pattern Steamflood Simulation Results, paper SPE-11079, presented at the 57th Annual Fall Technical Conference and Exhibition of the Society of Petroleum Engineers, New Orleans, LA, September 1982.

Corey, A.T. The Interrelation Between Gas and Oil Relative Permeabilities, Producers Monthly, 38-41, November 1954.

Cygan, R.T. The Solubility of Gases in $\mathrm{NaCl}$ Brine and a Critical Evaluation of Available Data, SAND90-2848, Sandia National Laboratories, Albuquerque, New Mexico, 1991. 
Dean, J.A. (ed.). Lange's Handbook of Chemistry, McGraw Hill, Thirteenth Edition, New York, 1985.

de Marsily, G. Quantitative Hydrogeology, Academic Press, Orlando, FL, 1986.

Doughty, C. and K. Pruess. A Similarity Solution for Two-Phase Water, Air and Heat Flow Near a Linear Heat Source in a Porous Medium, J. of Geophys. Res., 97 (B2), 1821-1838, 1992.

Duff, I.S. MA28-A Set of FORTRAN Subroutines for Sparse Unsymmetric Linear Equations, AERE Harwell Report R 8730, 1977.

Edlefsen, N.E. and A.B.C. Anderson. Thermodynamics of Soil Moisture, Hilgardia, 15 (2), 31-298, 1943.

Edwards, A.L. TRUMP: A Computer Program for Transient and Steady State Temperature Distributions in Multidimensional Systems, National Technical Information Service, National Bureau of Standards, Springfield, VA, 1972.

Falta, R.W., K. Pruess, I. Javandel and P.A. Witherspoon. Density - Driven Flow of Gas in the Unsaturated Zone Due to the Evaporation of Volatile Organic Compounds, Water. Resour. Res., Vol. 25, No. 10, pp. 2159 - 2169, 1989.

Falta, R.W., K. Pruess, S. Finsterle, and A. Battistelli. T2VOC User's Guide, Lawrence Berkeley Laboratory Report LBL-36400, Berkeley, CA, March 1995.

Fatt, I. and W.A. Klikoff. Effect of Fractional Wettability on Multiphase Flow Through Porous Media, AIME Transactions, 216, 246, 1959.

Faust, C.R. and J.W. Mercer. Summary of Our Research in Geothermal Reservoir Simulation, Proc. Workshop on Geothermal Reservoir Engineering, Stanford University, Stanford, CA, SGP-TR-12, 1975.

Finley, N.C. and M. Reeves. Swift Self-Teaching Curriculum, Report SAND81-0410, Sandia National Laboratory, Albuquerque, NM, 1982.

Grant, M.A. Permeability Reduction Factors at Wairakei, paper 77-HT-52, presented at AICHE-ASME Heat Transfer Conference, Salt Lake City, Utah, August 1977.

Hadgu, T. and D.H. Freeston. A Multi-Purpose Wellbore Simulator. Geothermal Resources Council Transactions, Vol. 14, pp. 1279 - 1286, 1990.

Hadgu, T., R.W. Zimmerman and G.S. Bodvarsson. Coupled Reservoir-Wellbore Simulation of Geothermal Reservoir Behavior. Geothermics, Vol. 24, No. 2, pp. 145-166, 1995.

Herbert, A.W., C.P. Jackson and D.A. Lever. Coupled Groundwater Flow and Solute Transport with Fluid Density Strongly Dependent on Concentration, Water Resour. 
Res., Vol. 24, No. 10, pp. 1781 - 1795, 1988.

Hirschfelder, J.O., C.F. Curtiss and R.B. Bird. Molecular Theory of Gases and Liquids, John Wiley \& Sons, New York, NY, 1954.

International Formulation Committee. A Formulation of the Thermodynamic Properties of Ordinary Water Substance, IFC Secretariat, Düsseldorf, Germany, 1967.

Jury, W.A., W.F. Spencer and W.J. Farmer. Behavior Assessment Model for Trace Organics in Soil: I. Model Description, J. Environ. Qual., Vol. 12, No. 4, pp. 558 564, 1983.

Kim, H.C., Bishnoi, P.R., Heideman, R.A., and Rizvi, S.S.H, Chem. Eng. Sci., 42, 16451653, 1987.

Klinkenberg, L.J. The Permeability of Porous Media to Liquids and Gases, in API Drilling and Production Practice, pp. 200-213, 1941.

Kruger, P. and H.J. Ramey, Jr. Stimulation and Reservoir Engineering of Geothermal Resources, Stanford Geothermal Program Report SGR-TR-1, Stanford University, Stanford, CA, 1974.

Lam, S.T., A. Hunsbedt, P. Kruger and K. Pruess. Analysis of the Stanford Geothermal Reservoir Model Experiments Using the LBL Reservoir Simulator, Geothermics, 17 (4), 595 - 605, LBL-25957, 1988.

Leverett, M.C. Capillary Behavior in Porous Solids, Trans. Soc. Pet. Eng. AIME, 142, 152-169, 1941.

Loomis, A.G. Solubilities of Gases in Water, in: International Critical Tables, Vol. III, E. W. Washburn, (ed.), McGraw-Hill, New York, NY, pp. 255-257, 1928.

Marion, G.M., Jakubowski, S.D., The compressibility of ice to $2.0 \mathrm{kbar}$, Cold Regions Science and Technology, Volume 38, Issues 2-3, p. 211-218, 2004.

de Marsily, G. Quantitative Hydrogeology, Academic Press, Orlando, FL, 1986.

Mason, E.A. and A.P. Malinauskas. Gas Transport in Porous Media: The Dusty Gas Model, Elsevier, Amsterdam, The Netherlands, 1983.

Michaelides, E.E. Thermodynamic Properties of Geothermal Fluids, Geothermal Resources Council Transactions, Vol. 5, pp. 361 - 364, 1981.

Miller, A.B. A Brine-Steam Properties Computer Program for Geothermal Energy Calculations, Lawrence Livermore National Laboratory Report UCRL-52495, Livermore, CA, June 1978.

Millington, R.J. and J.P. Quirk. Permeability of Porous Solids, Trans. Faraday Soc., 57, 1200-1207, 1961. 
Milly, P.C.D. Moisture and Heat Transport in Hysteretic, Inhomogeneous Porous Media: A Matric-Head Based Formulation and a Numerical Model, Water Resour. Res., Vol. 18, No. 3, pp. 489 - 498, 1982.

Moridis, G.J. Numerical studies of gas production from methane hydrates; Society of Petroleum Engineers Journal, Vol. 32, No.8, pp. 359-370, 2003.

Moridis, G.J., Numerical studies of gas production from methane hydrates; Society of Petroleum Engineers Reservoir Evaluation and Engineering, Vol. 27, No.3, pp. 175183, 2004.

Moridis, G.J. and T. Collett, Gas Production from Class 1 Hydrate Accumulations, in Recent Advances in the Study of Gas Hydrates, C. Taylor and J. Qwan, Editors, Kluwer Academic/Plenum Publishers (Section I, Chapter 6, pp. 75-88), 2004.

Moridis, G. and K. Pruess. TOUGH Simulations of Updegraff's Set of Fluid and Heat Flow Problems, Lawrence Berkeley Laboratory Report LBL-32611, Berkeley, CA, November 1992.

Moridis, G. and K. Pruess. Flow and Transport Simulations Using T2CG1, a Package of Conjugate Gradient Solvers for the TOUGH2 Family of Codes, Lawrence Berkeley Laboratory Report LBL-36235, Berkeley, CA, 1995.

Moridis, G. and K. Pruess. T2SOLV: An Enhanced Package of Solvers for the TOUGH2 Family of Reservoir Simulation Codes, Geothermics, Vol. 27, No. 4, pp. 415 - 444, 1998.

Moridis, G.J., Collett, T. S., Dallimore, S.R., Satoh, T., Hancock, S., and Weatherhill, B. Numerical studies of gas production from several methane hydrate zones at the Mallik site, Mackenzie Delta, Canada; Journal of Petroleum Science and Engineering, v. 43, p. 219-239, 2004 (also Report LBNL-50257, Lawrence Berkeley National Laboratory, Berkeley, California, 2002).

Moridis, G.J., Kowalsky, M.B., Pruess, K., TOUGH-Fx/HYDRATE v1.0 User's Manual: A Code for the Simulation of System Behavior in Hydrate-Bearing Geologic Media, Report LBNL-58950, Lawrence Berkeley National Laboratory, Berkeley, CA (2005).

Morrow, C., D. Lockner, D. Moore and J. Byerlee. Permeability of Granite in a Temperature Gradient, Journal of Geophysical Research, 86 (84), 3002-3008, April 1981.

Mualem, Y. A New Model for Predicting the Hydraulic Conductivity of Unsaturated Porous Media, Water Resour. Res., Vol. 12, No. 3, pp. 513 - 522, 1976.

Murray, L. and C. Gunn. Toward Integrating Geothermal Reservoir and Wellbore Simulation: TETRAD and WELLSIM, Proc. 15th NZ Geothermal Workshop, Auckland, New Zealand, 1993. 
Narasimhan, T.N. and P.A. Witherspoon. An Integrated Finite Difference Method for Analyzing Fluid Flow in Porous Media, Water Resour. Res., Vol. 12, No. 1, pp. 57 $-64,1976$.

Narasimhan, T.N., P.A. Witherspoon and A.L. Edwards. Numerical Model for SaturatedUnsaturated Flow in Deformable Porous Media, Part 2: The Algorithm, Water Resour. Res., 14 (2), 255-261, 1978.

Oldenburg, C.M. and K. Pruess. A Two-Dimensional Dispersion Module for the TOUGH2 Simulator, Lawrence Berkeley Laboratory Report LBL-32505, Berkeley, CA, September 1993.

Oldenburg, C.M. and K. Pruess. EOS7R: Radionuclide Transport for TOUGH2, Lawrence Berkeley Laboratory Report LBL-34868, Berkeley, CA, November 1995.

Oldenburg, C.M. and K. Pruess. Higher-Order Differencing for Phase Front Propagation in Geothermal Systems, Proc., Twenty-Third Workshop on Geothermal Reservoir Engineering, Stanford University, January 1998.

O'Sullivan, M.J. A Similarity Method for Geothermal Well Test Analysis, Water Resour. Res., Vol. 17, No. 2, pp. 390 - 398, 1981.

O’Sullivan, M.J., G.S. Bodvarsson, K. Pruess and M.R. Blakeley. Fluid and Heat Flow in Gas-Rich Geothermal Reservoirs, Society of Petroleum Engineers Journal, Vol. 25, No. 2, pp. 215 - 226, April 1985.

Pape, H., C. Clauser and J. Iffland. Permeability Prediction Based on Fractal Pore-Space Geometry, Geophysics, Vol. 64, No. 5, pp. 1447 - 1460, 1999.

Peaceman, D.W. Fundamentals of Numerical Reservoir Simulation, Elsevier, Amsterdam, The Netherlands, 1977.

Peaceman, D.W. Interpretation of Well-Block Pressures in Numerical Reservoir Simulation with Nonsquare Grid Blocks and Anisotropic Permeability, paper SPE10528, presented at the Sixth SPE Symposium on Reservoir Simulation of the Society of Petroleum Engineers, New Orleans, LA, January 1982.

Phillips, O.M. Flow and Reactions in Permeable Rocks, Cambridge University Press, Cambridge, New York, Melbourne,1991.

Pickens, J.F., R.W. Gillham and D.R. Cameron. Finite Element Analysis of the Transport of Water and Solutes in Tile-Drained Soils, J. of Hydrology, 40, 243-264, 1979.

Potter, J.M. and D.L. Brown. The Volumetric Properties of Aqueous Sodium Chloride Solutions, U. S. Geological Survey, Bulletin 1421-C, 1977.

Pritchett, J.W. Numerical Calculation of Multiphase Fluid and Heat Flow in 
Hydrothermal Reservoirs, Proc. Workshop on Geothermal Reservoir Engineering, Stanford University, Stanford, CA, pp. 201-205, SGP-TR-12, 1975.

Pritchett, J.W., M.H. Rice and T.D. Riney. Equation-of-State for Water-Carbon Dioxide Mixtures: Implications for Baca Reservoir, Report DOE/ET/27163-8, Systems, Science and Software, La Jolla, CA, February 1981.

Pruess, K. GMINC - A Mesh Generator for Flow Simulations in Fractured Reservoirs, Lawrence Berkeley Laboratory Report LBL-15227, Berkeley, CA, March 1983.

Pruess, K. TOUGH User's Guide, Nuclear Regulatory Commission Report NUREG/CR4645; also Lawrence Berkeley Laboratory Report LBL-20700, 1987.

Pruess, K. TOUGH2 - A General Purpose Numerical Simulator for Multiphase Fluid and Heat Flow, Lawrence Berkeley Laboratory Report LBL-29400, Berkeley, CA, 1991a.

Pruess, K. EOS7, An Equation-of-State Module for the TOUGH2 Simulator for TwoPhase Flow of Saline Water and Air, Lawrence Berkeley Laboratory Report LBL31114, Berkeley, CA, 1991b.

Pruess, K. Grid Orientation and Capillary Pressure Effects in the Simulation of Water Injection into Depleted Vapor Zones, Geothermics, Vol. 20, No. 5/6, pp. 257 - 277, 1991c.

Pruess, K., J.M. Zerzan, R.C. Schroeder and P.A. Witherspoon. Description of the Three-Dimensional Two-Phase Simulator SHAFT78 for Use in Geothermal Reservoir Studies, paper SPE-7699, presented at the Fifth SPE Symposium on Reservoir Simulation, Denver, CO, February 1979.

Pruess, K. and R.C. Schroeder. SHAFT 79 User's Manual, Lawrence Berkeley Laboratory Report LBL-10861, Berkeley, CA, March 1980.

Pruess, K., and T.N. Narasimhan. On Fluid Reserves and the Production of Superheated Steam from Fractured, Vapor-Dominated Geothermal Reservoirs, J. Geophys. Res., Vol. 87, No. B11, pp. 9329 - 9339, 1982.

Pruess, K. and G.S. Bodvarsson. A Seven-Point Finite Difference Method for Improved Grid Orientation Performance in Pattern Steam Floods, Proceedings, Seventh Society of Petroleum Engineers Symposium on Reservoir Simulation, Paper SPE12252, pp. 175 - 184, San Francisco, CA, 1983.

Pruess, K. and G.S. Bodvarsson. Thermal Effects of Reinjection in Geothermal Reservoirs with Major Vertical Fractures, J. Pet. Tech., Vol. 36, No. 10, pp. 1567 1578, 1984. 
Pruess, K. and T.N. Narasimhan. A Practical Method for Modeling Fluid and Heat Flow in Fractured Porous Media, Soc. Pet. Eng. J., Vol. 25, No. 1, pp. 14-26, February 1985.

Pruess, K., J.S.Y. Wang and Y.W. Tsang. On Thermohydrological Conditions Near High-Level Nuclear Wastes Emplaced in Partially Saturated Fractured Tuff. Part 2. Effective Continuum Approximation, Water Resour. Res., Vol. 26, No. 6, pp. 1249 - 1261, 1990.

Pruess, K., A. Simmons, Y.S. Wu and G. Moridis. TOUGH2 Software Qualification, Lawrence Berkeley National Laboratory Report LBL-38383, Berkeley, CA, February 1996.

Pruess, K., C. Oldenburg, and G.Moridis. TOUGH2 User's Guide - Version 2.0, Lawrence Berkeley Laboratory Report LBL-43134, Berkeley, CA, 1999.

Reeves, M., D. S. Ward, N. D. Johns and R. M. Cranwell. Theory and Implementation of Swift II, The Sandia Waste-Isolation Flow and Transport Model for Fractured Media, Report No. SAND83-1159, Sandia National Laboratory, Albuquerque, NM, 1986.

Richards, L.A. Capillary Conduction of Liquids Through Porous Mediums, Physics, Vol. 1, pp. $318-333,1931$.

Rutqvist J. and C.-F Tsang. A Study of Caprock Hydromechanical Changes Associated with $\mathrm{CO}_{2}$ Injection into a Brine Aquifer. Environmental Geology, Vol. 42, pp. 296$305,2002$.

Scheidegger, A. E. The Physics of Flow Through Porous Media, University of Toronto Press, Toronto and Buffalo, Third Edition, 1974.

Sleijpen, G.L.G. and D. Fokkema. BiCGSTAB(m) for Linear Equations Involving Unsymmetric Matrices with Complex Spectrum, Electronic Transactions on Numerical Analysis, Vol. 1, pp. 11 - 32, 1993.

Sloan, E.D., Clathrate Hydrates of Natural Gases, Marcel Dekker, Inc.: New York, NY, 1998.

Sutton, F.M. and A. McNabb. Boiling Curves at Broadlands Field, New Zealand, N. Z. J. Sci., Vol. 20, pp. 333 - 337, 1977.

Tanaka, H., Thermal expansivities of cubic ice I and ice VII, Journal of Molecular Structure: THEOCHEM, Volumes 461-462, Pages 561-567, 1999.

Thomas, G.W. Principles of Hydrocarbon Reservoir Simulation. International Human Resources Development Corporation, Boston, 1982. 
Tsang, Y.W. and K. Pruess. Further Modeling Studies of Gas Movement and Moisture Migration at Yucca Mountain, Nevada, Lawrence Berkeley Laboratory Report LBL-29127, Berkeley, CA, 1990.

Udell, K.S. and J.S. Fitch. Heat and Mass Transfer in Capillary Porous Media Considering Evaporation, Condensation, and Non-Condensible Gas Effects, paper presented at 23rd ASME/AIChE National Heat Transfer Conference, Denver, CO, 1985.

van der Vorst, H.A. Bi-CGSTAB: A Fast and Smoothly Converging Variant of BiCG in the Presence of Rounding Errors, SIAM J. Sci. Statist. Comput., Vol. 13, pp. 631 644, 1992.

van Genuchten, M.Th. A Closed-Form Equation for Predicting the Hydraulic Conductivity of Unsaturated Soils, Soil Sci. Soc. , Vol. 44, pp. 892 - 898, 1980.

Vargaftik, N.B. Tables on the Thermophysical Properties of Liquids and Gases, 2nd Ed., John Wiley \& Sons, New York, NY, 1975.

Vaughan, P.J. Analysis of Permeability Reduction During Flow of Heated, Aqueous Fluid Through Westerly Granite, in C.F. Tsang (ed.), Coupled Processes Associated with Nuclear Waste Repositories, pp. 529 - 539, Academic Press, New York, 1987.

Verma, A.K., K. Pruess, C.F. Tsang and P.A. Witherspoon. A Study of Two-Phase Concurrent Flow of Steam and Water in an Unconsolidated Porous Medium, Proc. 23rd National Heat Transfer Conference, Am. Society of Mechanical Engineers, Denver, CO, 135-143, 1985.

Verma, A. and K. Pruess. Thermohydrologic Conditions and Silica Redistribution Near High-Level Nuclear Wastes Emplaced in Saturated Geological Formations, J. of Geophys. Res., Vol. 93, No. B2, pp. 1159-1173, 1988.

Vinsome, P.K.W. and J. Westerveld. A Simple Method for Predicting Cap and Base Rock Heat Losses in Thermal Reservoir Simulators, J. Canadian Pet. Tech., Vol. 19, No. 3, pp. 87 - 90, July-September 1980.

Walker, W.R., J.D. Sabey, and D.R. Hampton. Studies of Heat Transfer and Water Migration in Soils, Final Report, Department of Agricultural and Chemical Engineering, Colorado State University, Fort Collins, CO, 80523, April 1981.

Warren, J.E. and P.J. Root. The Behavior of Naturally Fractured Reservoirs, Soc. Pet. Eng. J., Transactions, AIME, 228, 245-255, September 1963.

Webb, S.W. Gas-Phase Diffusion in Porous Media - Evaluation of an AdvectiveDispersive Formulation and the Dusty Gas Model for Binary Mixtures, J. Por. Media, Vol. 1, No. 2, pp. 187 - 199, 1998. 
Webb, S.W. and C.K. Ho. Review of Enhanced Vapor Diffusion in Porous Media, in: K. Pruess (ed.), Proceedings of the TOUGH Workshop '98, Lawrence Berkeley National Laboratory Report LBNL-41995, pp. 257 - 262, Berkeley, CA, 1998a.

Webb, S.W. and C.K. Ho. Enhanced Vapor Diffusion in Porous Media - LDRD Final Report, Sandia National Laboratories Report SAND98-2772, Albuquerque, NM, 1998b.

Wu, Y.S., K. Pruess and Z.X. Chen. Buckley-Leverett Flow in Composite Media, SPE Advanced Technology Series, Vol. 1, No. 2, pp. 36 - 42, 1993.

Xu, T., Y. Ontoy, P. Molling, N. Spycher, M. Parini and K. Pruess. Reactive Transport Modeling of Injection Well Scaling and Acidizing at Tiwi Field, Philippines, Geothermics, Vol. 33, No. 4, pp. 477 - 491, 2004.

Yanosik, J.L. and T.A. McCracken. A Nine-Point, Finite Difference Reservoir Simulator for Realistic Prediction of Adverse Mobility Ratio Displacements, Soc. Pet. Eng. J., $253-262,1979$. 


\section{Appendix A.}

\section{A1. Input File: Test_1T, Output File: Hydrate_Info_1T}

\begin{tabular}{|c|c|c|c|}
\hline d) & & & \\
\hline $1574074 \mathrm{E}-03$ & $.6170855 \mathrm{E}-05$ & $2.3888234 \mathrm{E}-05$ & $\overline{1} .61 \overline{7} 0855 \mathrm{E}-03$ \\
\hline $722222 \mathrm{E}-03$ & 9805379E-04 & $9257298 \mathrm{E}-04$ & 278441 \\
\hline & $735766 \mathrm{E}-04$ & $312954 \mathrm{E}$ & b1 \\
\hline $1018519 \mathrm{E}-03$ & $4142501 \mathrm{E}-04$ & $2429867 \mathrm{E}-03$ & $3298438 \mathrm{E}-$ \\
\hline $2731481 \mathrm{E}-02$ & $.0006471 \mathrm{E}-04$ & $.1818876 \mathrm{E}-03$ & $0301026 \mathrm{E}-$ \\
\hline .736111 & $1994 \mathrm{E}-04$ & $92 \mathrm{E}-\mathrm{-}-\mathrm{C}$ & $2 \mathrm{E}+00$ \\
\hline 2 & & 3 & \\
\hline $2.4305556 \mathrm{E}-02$ & $.9581837 \mathrm{E}-04$ & $1.1756147 \mathrm{E}-03$ & \\
\hline $3.3564815 \mathrm{E}-02$ & & & \\
\hline $4.2824074 \mathrm{E}-02$ & $1.8669362 \mathrm{E}-05$ & & $6248542 \mathrm{E}+00$ \\
\hline $5.2083333 \mathrm{E}-02$ & $8.8953002 \mathrm{E}-05$ & $1.3140493 \mathrm{E}-04$ & $1.6960166 \mathrm{E}+00$ \\
\hline $6.1342593 \mathrm{E}-02$ & $9.5474716 \mathrm{E}-05$ & $1.4103907 \mathrm{E}-04$ & $1.7723964 \mathrm{E}+00$ \\
\hline .06018 & 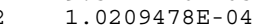 & & +00 \\
\hline .9 & 4 & & \\
\hline & & & \\
\hline & & & \\
\hline 2.0 & 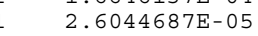 & 5 & +00 \\
\hline 2.0 & 05 & 05 & \\
\hline 2.28 & & & \\
\hline & & & \\
\hline $3.0208333 \mathrm{E}-01$ & $0187590 \mathrm{E}-04$ & $5049515 \mathrm{E}-04$ & $233334 \mathrm{E}+00$ \\
\hline 3. $7615741 \mathrm{E}-01$ & $1184297 \mathrm{E}-04$ & $6521891 \mathrm{E}-04$ & $34 \mathrm{E}+00$ \\
\hline $3.9467593 \mathrm{E}-01$ & 4 & 04 & \\
\hline $4.1319444 \mathrm{E}-01$ & 2.248 & 3.32 & +00 \\
\hline 4.31 & 1.13 & -05 & \\
\hline & & & \\
\hline & & & \\
\hline & & & \\
\hline & & & \\
\hline & & & \\
\hline & & & \\
\hline & & & \\
\hline & & & \\
\hline 7.6 & & $\begin{array}{l}0 \\
1\end{array}$ & \\
\hline & & & \\
\hline & & & \\
\hline & & & \\
\hline 9.7 & & & \\
\hline & & & \\
\hline & & & \\
\hline & & & \\
\hline & & & \\
\hline & & & \\
\hline & & & \\
\hline & & & \\
\hline & & & \\
\hline & & & \\
\hline & & P & \\
\hline & 5 & 05 & \\
\hline & 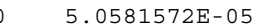 & $721121 \mathrm{E}-05$ & \\
\hline & & & $4 \mathrm{E}$ \\
\hline & & & $0 \mathrm{E}+01$ \\
\hline
\end{tabular}

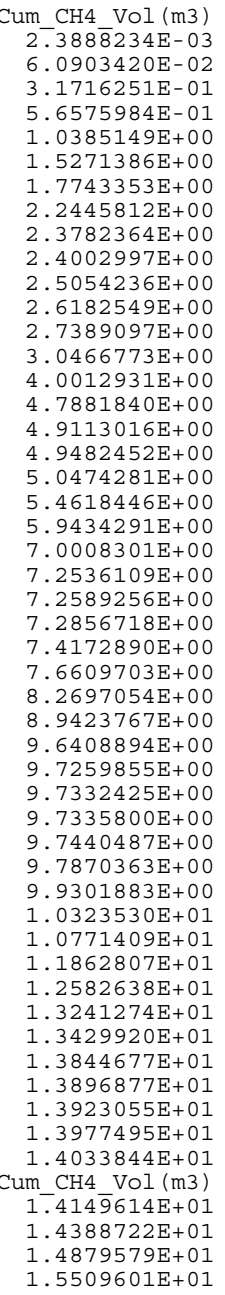

FreecH4 inRes (m3) $0.0000000 \mathrm{E}+00$
$5.1623371 \mathrm{E}-02$ $1.4044959 \mathrm{E}-01$
$1.7939587 \mathrm{E}-01$ . $1142200 \mathrm{E}-01$ $2.2629400 \mathrm{E}-01$ $2.3104015 \mathrm{E}-01$
$2.3472764 \mathrm{E}-01$ $1.6343697 \mathrm{E}-01$ . $3725767 \mathrm{E}-01$ $1.9879203 \mathrm{E}-01$ $2.4322904 \mathrm{E}-01$ . $4.1820989 \mathrm{E}-01$ $5955234 \mathrm{E}-01$ . $9170972 \mathrm{E}-01$ 3. $8938578 \mathrm{E}-01$ $4.0823857 \mathrm{E}-01$ $5.1997420 \mathrm{E}-01$ . $0453239 \mathrm{E}-01$ $.0121204 \mathrm{E}-01$ $.5779323 \mathrm{E}-01$ . $7269758 \mathrm{E}-01$ $7.8133775 \mathrm{E}-01$ $8.6434745 \mathrm{E}-01$ $9.2417118 \mathrm{E}-01$
$9.2969743 \mathrm{E}-01$ 8. $4143914 \mathrm{E}-01$ 8.2054372E-01 $7.9476674 \mathrm{E}-01$ $7.7762862 \mathrm{E}-01$ $.1044070 \mathrm{E}-01$ $9.9580352 \mathrm{E}-01$ $.1427285 \mathrm{E}+0$ . $1722893 \mathrm{E}+00$ $1.2392840 \mathrm{E}+00$ $.3470741 \mathrm{E}+00$ $.3560779 \mathrm{E}+00$ . $3707263 \mathrm{E}+00$ 1. $3818641 \mathrm{E}+00$ FreecH4 inRes (m3) $1.4038945 \mathrm{E}+00$ $.5182220 \mathrm{E}+00$
RemainHydr (kg) $1.3802331 \mathrm{E}+02$ 1. $3637458 \mathrm{E}+02$ $1.3507247 \mathrm{E}+02$ $1.3259627 \mathrm{E}+02$
$1.3003694 \mathrm{E}+02$ $.2874217 \mathrm{E}+02$ $2627911 \mathrm{E}+02$ $.2557904 \mathrm{E}+02$ $.2491286 \mathrm{E}+02$ . $2432187 \mathrm{E}+02$ $.2368990 \mathrm{E}+02$ $1.1707777 \mathrm{E}+02$ $1295617 \mathrm{E}+02$ $1.1231130 \mathrm{E}+02$ $.1211780 \mathrm{E}+02$ $.1159830 \mathrm{E}+02$ $1.0942766 \mathrm{E}+02$ $.0136673 \mathrm{E}+02$ $.0004271 \mathrm{E}+02$ $.9874779 \mathrm{E}+01$ $9.9185392 \mathrm{E}+01$ $9.7909033 \mathrm{E}+01$ . $1197256 \mathrm{E}+01$ 9. $1197256 \mathrm{E}+01$ $7092854 \mathrm{E}+01$ $.7054843 \mathrm{E}+01$ $.7053076 \mathrm{E}+01$ $.6998242 \mathrm{E}+01$ $.6023276 \mathrm{E}+01$ . $161713 \mathrm{E}+01$ 7. $5900564 \mathrm{E}+01$ $.2130223 \mathrm{E}+01$ $.5519885 \mathrm{E}+01$ $6.5246473 \mathrm{E}+01$ $4824211 \mathrm{E}+01$ $6.4529067 \mathrm{E}+01$ RemainHydr (kg) $6.3922682 \mathrm{E}+01$
$6.2670280 \mathrm{E}+01$ $6.0099257 \mathrm{E}+0$
Average P (Pa) $4.0127297 \mathrm{E}+06$ $4.0245574 \mathrm{E}+06$ $4.0127492 \mathrm{E}+06$ $4.0037762 \mathrm{E}+06$ $4.001492 \mathrm{E}+06$ $4.0014698+06$ $4.0006294 \mathrm{E}+0$ $4.0026979 \mathrm{E}+06$ $4.0091410 \mathrm{E}+06$ $4.0058418 \mathrm{E}+06$ $4.0039813 \mathrm{E}+06$ $4.0040381 \mathrm{E}+06$ $4.0033993 E+06$ $4.0038735 \mathrm{E}+06$ $4.0037022 \mathrm{E}+06$ $4.0035620 \mathrm{E}+06$ $4.0056911 \mathrm{E}+0$ $4.0050179 \mathrm{E}+06$ $4.0039383 \mathrm{E}+06$ $4.0030857 \mathrm{E}+06$ $4.0047496 \mathrm{E}+06$ $4.0063202 \mathrm{E}+06$ $4.0055867 \mathrm{E}+06$ $4.004936 \mathrm{E}+06$ $4.0037160 \mathrm{E}+06$ $4.0037919 \mathrm{E}+0$ $4.0048915 \mathrm{E}+06$ $4.0058420 \mathrm{E}+06$ $4.0063661 \mathrm{E}+06$ $4.0069892 \mathrm{E}+06$ $4.0075992 \mathrm{E}+06$ $4.0079434 \mathrm{E}+06$ $4.0080122 \mathrm{E}+06$ $4.0080017 \mathrm{E}+06$ 4. $0082735 \mathrm{E}+06$ $4.0085150 \mathrm{E}+06$ Average $\mathrm{P}(\mathrm{Pa})$ $4.0086860 \mathrm{E}+06$ $4.0088838 \mathrm{E}+06$ 4. $.0090656 \mathrm{E}+06$
Average $T$ (OC) $1.5223746 \mathrm{E}+00$ $1.5787711 \mathrm{E}+00$ $1.6469574 \mathrm{E}+00$ $1.7017886 \mathrm{E}+00$ 1.7583502 +00 . $3229140 \mathrm{E}+00$ $2.8376866 \mathrm{E}+00$ $3.1899845 \mathrm{E}+00$ . $5040747 \mathrm{E}+00$ . 4.5980727E+00 4. $9951004 \mathrm{E}+00$ $5.8382769 \mathrm{E}+00$ $6.0229516 \mathrm{E}+00$ $6.3406432 \mathrm{E}+00$ $6.7048667 \mathrm{E}+00$ $7.3960306 \mathrm{E}+00$ $7.5041695 \mathrm{E}+00$ $8.1850116 \mathrm{E}+00$ $8.6124803 \mathrm{E}+00$ $8.8962578 \mathrm{E}+00$ $9.3432237 \mathrm{E}+00$ $9.7140768 \mathrm{E}+00$ $.0075317 \mathrm{E}+01$ $1.0360329 \mathrm{E}+01$ $1.0430982 \mathrm{E}+01$
$1.0548445 \mathrm{E}+01$ $1.0730360 \mathrm{E}+01$ $1.0994757 \mathrm{E}+01$ $1.1766759 \mathrm{E}+01$ $1.2257619 \mathrm{E}+01$ $1.2999395 \mathrm{E}+01$ 1. $3685946 \mathrm{E}+01$ 1. $3824715 \mathrm{E}+01$ 1. $4061807 \mathrm{E}+01$ $1.4091176 \mathrm{E}+01$ $1.4132432 \mathrm{E}+01$ $1.4156692 \mathrm{E}+01$ Average $T$ (OC) $1.4284654 \mathrm{E}+01$ $1.4434362 \mathrm{E}+01$
$1.5015138 \mathrm{E}+01$ 
1. $9872685 \mathrm{E}+00$ $2.2835648 \mathrm{E}+00$
$2.5462963 \mathrm{E}+00$

3. $2659572 \mathrm{E}-05$ $4.1850895 \mathrm{E}-05$
$3.1019829 \mathrm{E}-05$
4. $8246026 \mathrm{E}-05$ $6.1823816 \mathrm{E}-05$ $4.5823733 \mathrm{E}-05$
$1.0917082 \mathrm{E}+01$ $1.2692615 \mathrm{E}+01$

$1.6127151 \mathrm{E}+01$ $1.7709840 \mathrm{E}+01$
$1.8750039 \mathrm{E}+01$

$.6139913 \mathrm{E}+00$ $.8986398 \mathrm{E}+00$
0 . $5274856 \mathrm{E}+01$ 3. $9826482 \mathrm{E}+01$

$4.0096244 \mathrm{E}+0$

$4.0098855 \mathrm{E}+06$

. $5531173 \mathrm{E}+01$

$1.6142699 \mathrm{E}+01$
$1.6960969 \mathrm{E}+01$ 


\section{A2. Input File: Test_1T, Output File: Conx_Time_Series_1T}

\begin{tabular}{|c|c|c|c|c|c|c|c|c|c|}
\hline $\begin{array}{l}\text { ime [days] }-\mathrm{G} \\
1.15741 \mathrm{E}-03\end{array}$ & $0.0000000 \mathrm{E}+00$ & $41516 \mathrm{E}-06$ & $-3.5073883 E+03$ & $0.00000000 \mathrm{E}+00$ & $4.4483768 \mathrm{E}-09$ & $4.4483768 \mathrm{E}-07$ & $2.6611403 \mathrm{E}-04$ & $0.0000000 \mathrm{E}+00$ & \\
\hline $\begin{array}{l}3.47222 \mathrm{E}-03 \\
3.4\end{array}$ & $8.3879276 \mathrm{E}-06$ & $5.0876048 \mathrm{E}-03$ & $-3.1950466 \mathrm{E}+03$ & $1.2388106 \mathrm{E}-05$ & $2.2144103 \mathrm{E}-05$ & $4.4292653 \mathrm{E}-03$ & $1.0164662 \mathrm{E}+00$ & $0.0000000 \mathrm{E}+00$ & \\
\hline $78704 \mathrm{E}-03$ & $5.5738109 \mathrm{E}-04$ & $7.9253039 \mathrm{E}-03$ & $.0595612 \mathrm{E}+03$ & $8.2319471 \mathrm{E}-04$ & $8.3843534 \mathrm{E}-04$ & $1.7211633 \mathrm{E}-01$ & $4636 E+00$ & $00 \mathrm{E}+00$ & \\
\hline $10185 E-03$ & $7.0040684 \mathrm{E}-04$ & $3.0635086 \mathrm{E}-03$ & $-3.1218694 \mathrm{E}+03$ & $1.0344288 \mathrm{E}-03$ & $1.0403027 \mathrm{E}-03$ & $3.8017688 \mathrm{E}-01$ & $3.2113701 \mathrm{E}+00$ & $0.0000000 \mathrm{E}+00$ & \\
\hline $27315 \mathrm{E}-02$ & $7.3671814 \mathrm{E}-04$ & $4.9582934 \mathrm{E}-04$ & $-3.1055455 E+03$ & $1.0880567 \mathrm{E}-03$ & $1.0890061 \mathrm{E}-03$ & $8.1577930 \mathrm{E}-01$ & $3.4094448 \mathrm{E}+00$ & $0.0000000 \mathrm{E}+00$ & \\
\hline $.73611 \mathrm{E}-02$ & $7.9218293 \mathrm{E}-04$ & $5.4822182 \mathrm{E}-04$ & $-3.1197619 \mathrm{E}+03$ & $1.1699724 \mathrm{E}-03$ & $1.1699724 \mathrm{E}-03$ & $1.2837683 \mathrm{E}+00$ & $3.6287335 \mathrm{E}+00$ & $0.0000000 \mathrm{E}+00$ & \\
\hline $1.96759 \mathrm{E}-02$ & $8.1083607 \mathrm{E}-04$ & $8.6925631 \mathrm{E}-04$ & $-3.1038394 \mathrm{E}+03$ & $1.1975212 \mathrm{E}-03$ & $1.1975212 \mathrm{E}-03$ & $1.5232725 \mathrm{E}+00$ & $3.8025848 \mathrm{E}+00$ & $0.0000000 \mathrm{E}+00$ & \\
\hline $43056 \mathrm{E}-02$ & $7.8028489 \mathrm{E}-04$ & $1.1196963 \mathrm{E}-03$ & $-2.8792807 \mathrm{E}+03$ & $1.1524003 \mathrm{E}-03$ & $1.1524003 \mathrm{E}-03$ & $1.9842326 \mathrm{E}+00$ & $4.2504633 \mathrm{E}+00$ & $0.0000000 \mathrm{E}+00$ & \\
\hline $35648 \mathrm{E}-02$ & $1.7391341 \mathrm{E}-04$ & $2.0039315 E-03$ & $-2.4704505 \mathrm{E}+03$ & $2.5682776 \mathrm{E}-04$ & $2.5682776 \mathrm{E}-04$ & $2.1896948 \mathrm{E}+00$ & $5.8536085 \mathrm{E}+00$ & $0.0000000 \mathrm{E}+00$ & \\
\hline $.28241 \mathrm{E}-02$ & $4.2511564 \mathrm{E}-05$ & $6.9879267 \mathrm{E}-04$ & $-1.9892072 \mathrm{E}+03$ & $6.2772322 \mathrm{E}-05$ & $6.2772322 \mathrm{E}-05$ & $2.2399127 \mathrm{E}+00$ & $6.4126426 \mathrm{E}+00$ & $0.0000000 \mathrm{E}+00$ & \\
\hline $20833 \mathrm{E}-02$ & $3.8307854 \mathrm{E}-05$ & $1.5436836 \mathrm{E}-03$ & $-1.5601638 \mathrm{E}+03$ & $5.6559662 \mathrm{E}-05$ & $5.8758370 \mathrm{E}-05$ & $2.2869194 \mathrm{E}+00$ & $7.6463988 \mathrm{E}+00$ & $0.0000000 \mathrm{E}+00$ & \\
\hline $13426 \mathrm{E}-02$ & $5.8057707 \mathrm{E}-05$ & $1.1342974 \mathrm{E}-03$ & $-1.4202176 \mathrm{E}+03$ & $8.5711353 \mathrm{E}-05$ & $8.7245286 \mathrm{E}-05$ & $2.3567156 \mathrm{E}+00$ & $8.5530059 \mathrm{E}+00$ & $0.0000000 \mathrm{E}+00$ & \\
\hline $019 \mathrm{E}-02$ & $7.7076230 \mathrm{E}-05$ & $6.8600119 \mathrm{E}-04$ & $-1.3239521 \mathrm{E}+03$ & $1.1377881 \mathrm{E}-04$ & $1.1466994 \mathrm{E}-04$ & $2.4484516 \mathrm{E}+00$ & 9.101324 & & \\
\hline $204 \mathrm{E}-02$ & $1.1108516 \mathrm{E}-04$ & $4.0454205 \mathrm{E}-04$ & $-1.1731073 \mathrm{E}+03$ & $1.6396044 \mathrm{E}-04$ & $1.6445965 \mathrm{E}-04$ & $2.7115870 \mathrm{E}+00$ & $9.7480509 \mathrm{E}+00$ & .0000000 & \\
\hline $26157 \mathrm{E}-01$ & $1.7837468 \mathrm{E}-04$ & $4.0007722 \mathrm{E}-04$ & $-9.9299629 \mathrm{E}+02$ & $2.6323772 \mathrm{E}-04$ & $2.6370805 \mathrm{E}-04$ & $3.5554528 \mathrm{E}+00$ & $1.1027279 \mathrm{E}+01$ & +00 & \\
\hline $63194 \mathrm{E}-01$ & $1.5662421 \mathrm{E}-04$ & $2.4469681 E-05$ & $-9.0746435 \mathrm{E}+02$ & $2.3110709 \mathrm{E}-04$ & $2.3113485 \mathrm{E}-04$ & $4.2950843 E+00$ & $1.1105522 \mathrm{E}+01$ & $0.0000000 \mathrm{E}+00$ & \\
\hline $.00231 \mathrm{E}-01$ & $4.0592944 \mathrm{E}-05$ & $4.9356938 \mathrm{E}-04$ & $-8.9300129 \mathrm{E}+02$ & $5.9887923 \mathrm{E}-05$ & $5.9887923 \mathrm{E}-05$ & $4.4867257 \mathrm{E}+00$ & $1.2684944 \mathrm{E}+01$ & $0.0000000 \mathrm{E}+00$ & \\
\hline $.09491 \mathrm{E}-01$ & $3.3370720 \mathrm{E}-05$ & $1.0045006 \mathrm{E}-04$ & $-7.9308829 \mathrm{E}+02$ & $4.9231156 \mathrm{E}-05$ & $4.9231156 \mathrm{E}-05$ & $4.5261106 \mathrm{E}+00$ & $1.2765304 \mathrm{E}+01$ & $0.0000000 \mathrm{E}+00$ & \\
\hline $28009 \mathrm{E}-01$ & $3.3822545 \mathrm{E}-05$ & $2.1696277 \mathrm{E}-04$ & $-7.0225550 \mathrm{E}+02$ & $4.9895072 \mathrm{E}-05$ & $5.0129366 \mathrm{E}-05$ & $4.6063176 \mathrm{E}+00$ & $1.3112191 \mathrm{E}+01$ & 0.00000001 & \\
\hline $2.65046 \mathrm{E}-01$ & $6.3748648 \mathrm{E}-05$ & $6.5123346 \mathrm{E}-04$ & $-5.8122630 \mathrm{E}+02$ & $9.4035425 \mathrm{E}-05$ & $9.4730003 \mathrm{E}-05$ & $4.9094536 \mathrm{E}+00$ & $1.5194633 \mathrm{E}+01$ & $0.0000000 \mathrm{E}+00$ & \\
\hline $3.02083 \mathrm{E}-01$ & $8.4577431 \mathrm{E}-05$ & $3.9556188 \mathrm{E}-04$ & $-5.6835982 \mathrm{E}+02$ & $1.2475193 \mathrm{E}-04$ & $1.2516940 \mathrm{E}-04$ & $5.3099957 \mathrm{E}+00$ & $1.6459527 \mathrm{E}+01$ & $0.0000000 \mathrm{E}+00$ & \\
\hline $.76157 \mathrm{E}-01$ & $1.0131936 \mathrm{E}-04$ & $1.3977700 \mathrm{E}-04$ & $-5.4261458 \mathrm{E}+02$ & $1.4943298 \mathrm{E}-04$ & $1.4957848 \mathrm{E}-04$ & $6.2672979 \mathrm{E}+00$ & $1.7353469 \mathrm{E}+01$ & 0.000000 & \\
\hline $3.94676 \mathrm{E}-01$ & $1.0013104 \mathrm{E}-04$ & $2.6052050 \mathrm{E}-05$ & $-5.4491212 \mathrm{E}+02$ & $1.4767701 \mathrm{E}-04$ & 1.47704 & $6.5036244 \mathrm{E}+00$ & $1.7395123 \mathrm{E}+01$ & 0.0000 & \\
\hline $4.13194 \mathrm{E}-01$ & $4.5661752 \mathrm{E}-05$ & $1.4241880 \mathrm{E}-03$ & $-7.9922979 \mathrm{E}+02$ & $6.7340617 \mathrm{E}-05$ & $6.7340617 \mathrm{E}-05$ & $6.6113694 \mathrm{E}+00$ & $1.9673824 \mathrm{E}+01$ & 0.000 & \\
\hline 1 & $2.9657881 \mathrm{E}-05$ & $6.1874946 \mathrm{E}-04$ & $-6.2945374 \mathrm{E}+02$ & $4.3737346 \mathrm{E}$ & $6 \mathrm{E}-05$ & 6.6813 & $3 \mathrm{E}+01$ & .00 & \\
\hline $\begin{array}{l}1750 \\
8750\end{array}$ & $\begin{array}{l}2.4651731 \mathrm{E}-05 \\
2.476173 \mathrm{~s}\end{array}$ & $\begin{array}{l}6.18 \\
6.7316199 \mathrm{E}-05\end{array}$ & $-4.8154967 \mathrm{E}+02$ & $\begin{array}{l}4.3515717 \\
3.6515\end{array}$ & $3.6584525 \mathrm{E}-05$ & $\begin{array}{l}6.68134 \\
6.79841\end{array}$ & $2.0879086 \mathrm{E}+01$ & .00 & \\
\hline $5787 \mathrm{E}-01$ & $3.3785106 \mathrm{E}-05$ & $5.1327388 \mathrm{E}-04$ & $-3.9848072 \mathrm{E}+02$ & $4.9821442 \mathrm{E}-0$ & $5.0344873 \mathrm{E}-05$ & $6.9595232 \mathrm{E}+00$ & $2.2520429 \mathrm{E}+01$ & $.0000000 \mathrm{E}+00$ & \\
\hline $.79861 \mathrm{E}-01$ & $5.0874205 \mathrm{E}-05$ & $3.4132450 \mathrm{E}-04$ & $-3.9204587 \mathrm{E}+02$ & $7.5018291 \mathrm{E}-05$ & $7.5364170 \mathrm{E}-05$ & $7.4418539 \mathrm{E}+00$ & $2.4703407 \mathrm{E}+01$ & $0.0000000 \mathrm{E}+00$ & \\
\hline $5.53935 \mathrm{E}-01$ & $6.1848646 \mathrm{E}-05$ & $1.8049933 \mathrm{E}-04$ & $-3.8816017 \mathrm{E}+02$ & $9.1196772 \mathrm{E}-05$ & $9.1378624 \mathrm{E}-05$ & $8.0266771 \mathrm{E}+00$ & $2.5857815 \mathrm{E}+01$ & 0.000 & \\
\hline $7.28009 \mathrm{E}-01$ & $6.6972518 \mathrm{E}-05$ & $9.1628618 \mathrm{E}-05$ & $-3.7954475 \mathrm{E}+02$ & $9.8748239 \mathrm{E}-05$ & $9.8840131 \mathrm{E}-05$ & $8.6592539 \mathrm{E}+00$ & $2.6443840 \mathrm{E}+01$ & $0.0000000 \mathrm{E}+00$ & \\
\hline $7.37269 \mathrm{E}-01$ & $6.6765915 \mathrm{E}-05$ & $3.9953546 \mathrm{E}-05$ & $-3.8504487 E+02$ & $9.8443110 \mathrm{E}-05$ & $9.8483154 \mathrm{E}-05$ & $8.7380404 \mathrm{E}+00$ & $2.6475781 \mathrm{E}+01$ & $0.0000000 \mathrm{E}+00$ & \\
\hline 7. $55787 \mathrm{E}-01$ & $3.9990299 \mathrm{E}-05$ & $1.2253461 \mathrm{E}-03$ & $-6.1463664 \mathrm{E}+02$ & $5.8962206 \mathrm{E}-05$ & $62206 \mathrm{E}-05$ & $8.8323800 \mathrm{E}+00$ & $2.8436335 \mathrm{E}+01$ & 0.00 & \\
\hline $7 E-01$ & $075 E-05$ & $1.1651530 \mathrm{E}-03$ & $2638 \mathrm{E}+02$ & 5.2 & & $8.8533561 \mathrm{E}+00$ & 2.8 & 0.0 & \\
\hline $7.69676 \mathrm{E}-01$ & $3.0563146 \mathrm{E}-05$ & $7.3786859 \mathrm{E}-04$ & $-5.1425786 \mathrm{E}+02$ & $4.5062041 \mathrm{E}-05$ & $2041 E-05$ & $7 E+00$ & 2.9 & 0.00 & \\
\hline 7.8 & 2.55 & $2.7102227 \mathrm{E}-04$ & -4.2 & 3.7 & 3.7 & 8.94 & 2.99 & 0.00 & \\
\hline & 2.4 & 1.6 & & 3.5 & 3.5 & 9.0 & 3.04 & 0.0 & \\
\hline $3.99306 \mathrm{~F}$ & $3.0629447 \mathrm{E}-05$ & $3.0179458 \mathrm{E}-04$ & $-3.0505680 \mathrm{E}+02$ & $4.5158398 \mathrm{E}-05$ & 4.54 & $9.3551064 \mathrm{E}+00$ & $3.2373729 \mathrm{E}+01$ & 0.000 & \\
\hline $73380 \mathrm{E}-01$ & 3.80843 & 2.308433 & -3.0252 & 5.61 & 5.637 & $9.7159191 \mathrm{E}+00$ & $3.3850134 \mathrm{E}+01$ & $0.0000000 \mathrm{E}$ & \\
\hline $153 \mathrm{E}+00$ & $4.9593564 \mathrm{E}-05$ & $1.6632496 \mathrm{E}-04$ & $4741 \mathrm{E}+02$ & 7.42112470 & 7.327 & $1.0653872 \mathrm{E}+01$ & $3.5977672 \mathrm{E}+01$ & 0.0000000 & \\
\hline $68 \mathrm{E}+00$ & $3.6418637 \mathrm{E}-05$ & 7.69549 & -2. & & 5.3687 & $.078 E+01$ & $6076174 \mathrm{E}+01$ & .0000000 & \\
\hline $782 \mathrm{E}+00$ & 3.12 & 6.35 & -2 . & 4. & & 1.19 & 3.6 & & \\
\hline & 3.25 & 1. & & 4 & 4. & 1.20 & 3.74 & 0.0 & \\
\hline 1.5 & $3.5970976 \mathrm{E}-05$ & $1.8943275 \mathrm{E}-04$ & $-2.4064187 \mathrm{E}+02$ & $5.3025740 \mathrm{E}$ & $5.3211030 \mathrm{E}-05$ & $1.2426721 \mathrm{E}+01$ & $3.8676407 \mathrm{E}+01$ & $0.0000000 \mathrm{E}$ & \\
\hline 1.5 & $3.6391302 \mathrm{E}-05$ & $1.8471205 \mathrm{E}-04$ & $-2.4067285 E+02$ & $5.3645279 \mathrm{E}$ & $5.3825927 \mathrm{E}-05$ & $1.2469781 \mathrm{E}+01$ & $3.8824078 E+01$ & $0.0000000 \mathrm{E}$ & \\
\hline & $339 E-05$ & $1.8184844 \mathrm{E}-04$ & -2.4 & 5.39483761 & 5.41 & $1.2491432 \mathrm{E}+01$ & $3.8896770 \mathrm{E}+01$ & $0.0000000 \mathrm{E}+00$ & \\
\hline $1.55208 \mathrm{E}+00$ & $3.7282774 \mathrm{E}-05$ & $2.1344807 \mathrm{E}-04$ & $-2.3463255 E+02$ & $5.4959317 \mathrm{E}-05$ & $5.5168031 \mathrm{E}-05$ & $1.2535566 \mathrm{E}+01$ & $3.9067415 \mathrm{E}+01$ & $0.0000000 \mathrm{E}+00$ & \\
\hline $1.56134 \mathrm{E}+00$ & $3.8108756 \mathrm{E}-05$ & $2.3751696 \mathrm{E}-04$ & $-2.2986541 \mathrm{E}+02$ & $5.6176859 \mathrm{E}-05$ & $5.6409085 \mathrm{E}-05$ & $1.2580693 \mathrm{E}+01$ & 3.92573 & 0.00 & \\
\hline $6 \mathrm{E}+00$ & $3.9508495 \mathrm{E}-05$ & $2.3187467 \mathrm{E}-04$ & $-2.2947717 \mathrm{E}+02$ & $5.8240111 \mathrm{E}-05$ & $71 \mathrm{E}-05$ & $1.2674240 \mathrm{E}+01$ & $3.9628057 \mathrm{E}+01$ & $0.0000000 \mathrm{E}$ & \\
\hline & $4.1614629 \mathrm{E}-05$ & -04 & $-2.3014276 \mathrm{E}+02$ & $6.1344476 \mathrm{E}-05$ & $6.1550013 \mathrm{E}-05$ & $1.2871200 \mathrm{E}+01$ & $4.0300816 \mathrm{E}+01$ & $0.0000000 \mathrm{E}$ & \\
\hline & $4.4035777 \mathrm{E}-05$ & $\begin{array}{l}10 \mathrm{E}-04 \\
\end{array}$ & $-2.3090726 \mathrm{E}+02$ & $6.4912777 \mathrm{E}-05$ & $6.5078345 \mathrm{E}-05$ & $1.3287702 \mathrm{E}+01$ & $4.1385904 \mathrm{E}+01$ & $0.0000000 \mathrm{~F}$ & \\
\hline & & & & & & & & & \\
\hline & & & & & & & & & \\
\hline & & & & & & & & & \\
\hline $4630 \mathrm{E}+00$ & $2.9496765 \mathrm{E}-05$ & $.9162607 \mathrm{E}-06$ & $-2.0854658 \mathrm{E}+02$ & $4.3476464 \mathrm{E}-05$ & $4.3480247 \mathrm{E}-05$ & $1.6773029 \mathrm{E}+01$ & $4.6122989 \mathrm{E}+01$ & $0.0000000 \mathrm{E}+00$ & \\
\hline
\end{tabular}




\section{A3. Input File: Test_1P_Ice, Output File: Hydrate_Info_1P_Ice}

\begin{tabular}{|c|c|c|c|c|}
\hline & & & & \\
\hline $574074 \mathrm{E}-05$ & $5.0109078 \mathrm{E}-02$ & $7.4023134 \mathrm{E}-02$ & $\overline{5} .01 \overline{0} 9078 \mathrm{E}-\bar{C}$ & $7.402 \overline{3} 134 \mathrm{E}-02$ \\
\hline $2.3148148 E-05$ & $6.0215747 \mathrm{E}-02$ & $8.8953110 \mathrm{E}-02$ & $1.1032482 \mathrm{E}-01$ & $1.6297624 \mathrm{E}-01$ \\
\hline $4.6296296 \mathrm{E}-05$ & $9.2941321 \mathrm{E}-02$ & $1.3729664 \mathrm{E}-01$ & $2.9620747 \mathrm{E}-01$ & -01 \\
\hline $.9444444 \mathrm{E}-05$ & $0034397 \mathrm{E}-01$ & $1.4823213 \mathrm{E}-01$ & $41 \mathrm{E}-01$ & \\
\hline $1.1574074 \mathrm{E}-04$ & $1.3815199 \mathrm{E}-01$ & $2.0408365 \mathrm{E}-01$ & $1.0495034 \mathrm{E}+00$ & $03684 E+00$ \\
\hline $1.6203704 \mathrm{E}-04$ & $1.9333136 \mathrm{E}-01$ & $2.8559682 \mathrm{E}-01$ & $1.8228288 \mathrm{E}+00$ & $2.6927557 \mathrm{E}+00$ \\
\hline $2.5462963 \mathrm{E}-04$ & $4.9870013 \mathrm{E}-02$ & $7.3669979 \mathrm{E}-02$ & $2.2217889 \mathrm{E}+00$ & $3.2821155 \mathrm{E}+00$ \\
\hline $3.4722222 \mathrm{E}-04$ & $2.9467972 \mathrm{E}-02$ & $4.3531266 \mathrm{E}-02$ & $2.4575327 \mathrm{E}+00$ & $3.6303656 \mathrm{E}+00$ \\
\hline $4.3981481 \mathrm{E}-04$ & $2.6466071 \mathrm{E}-02$ & $3.9096738 \mathrm{E}-02$ & $2.6692613 \mathrm{E}+00$ & $3.9431395 \mathrm{E}+00$ \\
\hline $6.2500000 \mathrm{E}-04$ & $3.1700276 \mathrm{E}-02$ & $4.6828915 \mathrm{E}-02$ & $.1764657 \mathrm{E}+00$ & $4.6924022 \mathrm{E}+00$ \\
\hline $1018519 \mathrm{~F}=04$ & $4.5786713 \mathrm{E}-02$ & $6.7637964 \mathrm{E}-02$ & $9090531 E+00$ & \\
\hline & .953 & 1 & & \\
\hline .5 & .4340 & & & \\
\hline & $.663^{\circ}$ & & & \\
\hline $1.8287037 \mathrm{E}-03$ & 3.147333 & 4.64936 & $7 \mathrm{E}+00$ & 9 \\
\hline $2.1990741 \mathrm{E}-03$ & $.0926381 \mathrm{E}-02$ & $3.0913287 \mathrm{E}-02$ & $9 E+00$ & 1 \\
\hline $2.5694444 \mathrm{E}-03$ & $1.6049442 \mathrm{E}-02$ & $2.3708878 \mathrm{E}-02$ & $5329071 \mathrm{E}+00$ & 1 \\
\hline $2.9398148 \mathrm{E}-03$ & $1.9861347 \mathrm{E}-02$ & $2.9339976 \mathrm{E}-02$ & $1684702 \mathrm{E}+00$ & $1.2066791 \mathrm{E}+$ \\
\hline $3.6805556 \mathrm{E}-03$ & $1.8952497 \mathrm{E}-02$ & $2.7997387 \mathrm{E}-02$ & $9.3814300 \mathrm{E}+00$ & $1.3858624 \mathrm{E}+$ \\
\hline $4.4212963 \mathrm{E}-03$ & $2.1858240 \mathrm{E}-02$ & $3.2289866 \mathrm{E}-02$ & $1.0780357 \mathrm{E}+01$ & $1.5925175 \mathrm{E}+01$ \\
\hline $5.1620370 \mathrm{E}-03$ & $1.7712118 \mathrm{E}-02$ & $2.6165050 \mathrm{E}-02$ & $1.1913933 \mathrm{E}+01$ & $1.7599738 \mathrm{E}+01$ \\
\hline 5.9 & .8 & 2.6 & & 1.9 \\
\hline 6.64 & 1.85 & 2.74 & & 2. \\
\hline & & & & \\
\hline & & & & \\
\hline & & & & \\
\hline & & & & \\
\hline & & & & \\
\hline $1.1087963 \mathrm{E}-02$ & 0 & 0.00 & +01 & $\mathrm{E}+01$ \\
\hline 1.25 & & & & 2.63 \\
\hline 1.55 & & & & \\
\hline 1.84 & & & & 2. \\
\hline 2.14 & 2 & 0 & 01 & 2.63 \\
\hline 2.4 & & & & 2.63 \\
\hline & & & & \\
\hline 4.2 & 0.0 & & & +01 \\
\hline & & & & \\
\hline & & & & \\
\hline & & & & +01 \\
\hline & & & & $\begin{array}{l}+01 \\
+01\end{array}$ \\
\hline & & & & \\
\hline & & & & \\
\hline & & & & $365070 \mathrm{E}+01$ \\
\hline 1.2 & & & & $5070 \mathrm{E}+$ \\
\hline & & 0 & 1.7847 & 2.636 \\
\hline 2.19 & $0.0000000 \mathrm{E}+00$ & 0.00 & $1.7847520 \mathrm{E}+01$ & $2.6365070 \mathrm{E}$ \\
\hline & & & & \\
\hline & & & 1.7 & +01 \\
\hline 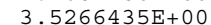 & 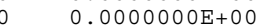 & & $1.78 \quad 30-5$ & 2.636 \\
\hline & & & & \\
\hline & & & & \\
\hline & & & & \\
\hline & & & & \\
\hline & & & & \\
\hline
\end{tabular}

reeCH4 inRes ( $\mathrm{m} 3$ ) $7.0379416 \mathrm{E}-02$
$1.2015934 \mathrm{E}-01$ $1.7727946 \mathrm{E}-01$ $2.1224249 \mathrm{E}-01$ 2. $8607020 \mathrm{E}-01$ $3.4567922 \mathrm{E}-01$
$3.6215981 \mathrm{E}-01$ $.1361859 \mathrm{E}-01$ $4.7135039 \mathrm{E}-01$ $5.9275387 \mathrm{E}-01$ 7. $0919324 \mathrm{E}-01$ $8.5397618 \mathrm{E}-01$ $1.0270450 \mathrm{E}+00$ $1.1030952 \mathrm{E}+00$ . $1.3086037 \mathrm{E}+00$ $.3872025 \mathrm{E}+00$ $1.4520401 \mathrm{E}+00$ $1.4051348 \mathrm{E}+00$
$1.4351187 \mathrm{E}+00$ $.4351187 \mathrm{E}+00$
$1.5075471 \mathrm{E}+00$ $1.5962916 \mathrm{E}+00$ $1.6391791 \mathrm{E}+00$ $1.6057641 \mathrm{E}+00$ $1.2395189 \mathrm{E}+00$
$8.6310333 \mathrm{E}-01$ $6.7495103 \mathrm{E}-01$ $5.2877373 \mathrm{E}-01$
$4.4207659 \mathrm{E}-01$ $4.1036127 \mathrm{E}-01$ $3.9616386 \mathrm{E}-01$ 3. $8833577 \mathrm{E}-01$ 3. $7996943 \mathrm{E}-01$ $3.7007549 \mathrm{E}-01$ 3. $5692870 \mathrm{E}-01$ 3.1162625E-01 $2.7307366 \mathrm{E}-01$ .5641539E-0 2. $2984061 \mathrm{E}-01$ 2. $0842766 \mathrm{E}-01$ $1.7869530 \mathrm{E}-01$ $1.4612385 \mathrm{E}-01$ $1.3317165 \mathrm{E}-01$ $1.2376962 \mathrm{E}-01$ $1.1127609 \mathrm{E}-01$ 9.7429816E-02 8. $8660257 \mathrm{E}-02$ 8. $2451263 \mathrm{E}-02$ $7.6579155 \mathrm{E}-02$
RemainHydr (kg) $1.3724187 \mathrm{E}+02$ 1. $3425077 \mathrm{E}+02$ 1. $2997495 \mathrm{E}+02$ . $2399134 \mathrm{E}+02$ $.2090437 \mathrm{E}+02$ $1.1908030 \mathrm{E}+02$ $.1351755 \mathrm{E}+02$ $0784914 \mathrm{E}+02$ $.0053661 \mathrm{E}+02$ $.4510006 \mathrm{E}+01$ $9.2861146 \mathrm{E}+01$ $8.8964738 \mathrm{E}+01$ $9809503 \mathrm{E}+01$ $.4891822 \mathrm{E}+01$ . $5506523 \mathrm{E}+01$ $5.4682298 \mathrm{E}+01$ $3.6868962 \mathrm{E}+01$ $.7681963 \mathrm{E}+0$ $8570420 \mathrm{E}+01$ $9499471 \mathrm{E}+00$ $0.0000000 \mathrm{E}+00$ $0.0000000 \mathrm{E}+00$ $.0000000 \mathrm{E}+00$ $0.0000000 \mathrm{E}+00$ $.0000000 \mathrm{E}+00$ $0.0000000 \mathrm{E}+00$ $.0000000 \mathrm{E}+00$ $0.0000000 \mathrm{E}+00$ $0.0000000 \mathrm{E}+00$ $0.0000000 \mathrm{E}+00$ $0.0000000 \mathrm{E}+00$ $0.0000000 \mathrm{E}+00$ $0.0000000 \mathrm{E}+00$ $0.0000000 \mathrm{E}+00$ $.0000000 \mathrm{E}+00$ $0.0000000 \mathrm{E}+00$ $.0000000 \mathrm{E}+00$ $0.0000000 \mathrm{E}+00$ $.0000000 \mathrm{E}+00$ $0.0000000 \mathrm{E}+00$ $0.0000000 \mathrm{E}+00$ $0.0000000 \mathrm{E}+00$ $0.0000000 \mathrm{E}+00$
Average P (Pa) $5.7615344 \mathrm{E}+06$ $.6090182 \mathrm{E}+06$ $.4637230 \mathrm{E}+06$ $2030309 \mathrm{E}+06$ $4.8906197 \mathrm{E}+06$ $4.6851152 \mathrm{E}+06$ $4.3456619 \mathrm{E}+06$ 4. $2446243 \mathrm{E}+06$ $3.9236926 \mathrm{E}+06$ . $7014951 \mathrm{E}+0$ 3. $6667519 \mathrm{E}+0$ $3.6044038 \mathrm{E}+06$ 3. $1420433 \mathrm{E}+06$ $3.0396753 \mathrm{E}+06$ $2.7298232 \mathrm{E}+06$ $2.5460864 \mathrm{E}+06$ $2.2843636 \mathrm{E}+0$ $2.1460296 \mathrm{E}+0$ $2.0762164 \mathrm{E}+06$ $1.9918740 \mathrm{E}+06$ 1. $8827004 \mathrm{E}+06$ $1.4581248 \mathrm{E}+0$ $1.0387363 \mathrm{E}+06$ $8.2239295 \mathrm{E}+05$ $5.5403373 \mathrm{E}+05$ $5.2266175 \mathrm{E}+05$
$5.1191803 \mathrm{E}+05$ $5.0810671 \mathrm{E}+05$ 5. $0661310 \mathrm{E}+05$ $5.0658246 \mathrm{E}+05$ $5.0697809 \mathrm{E}+05$ $5.0735342 \mathrm{E}+05$ $5.0769837 \mathrm{E}+0$ $5.0768705 \mathrm{E}+05$ $5.0761468 \mathrm{E}+05$ $5.0753021 \mathrm{E}+05$ $5.0682349 \mathrm{E}+05$ $5.0655876 \mathrm{E}+05$ $5.0632544 \mathrm{E}+05$ $5.0596423 \mathrm{E}+0.5$ $5.0548755 \mathrm{E}+05$ $5.0515616 \mathrm{E}+05$ $5.0490113 \mathrm{E}+05$ 5. $0464791 \mathrm{E}+05$
Average $\mathrm{T}$ (OC) $7.0505703 \mathrm{E}+00$

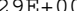
$6.5645408 \mathrm{E}+00$ $6.3044863 \mathrm{E}+00$ $5.9123129 \mathrm{E}+00$ $5.6230062 \mathrm{E}+00$ $5.0034602 \mathrm{E}+00$ $4.7826296 \mathrm{E}+00$ $4.1650482 \mathrm{E}+00$ $3.6806840 \mathrm{E}+00$ $3.5912855 \mathrm{E}+00$ $3.4391179 \mathrm{E}+00$ 1.0420118E+00 $5.4658991 \mathrm{E}-01$ $6.8532400 \mathrm{E}-02$ $1.1180973 \mathrm{E}-02$ $1.0000000 \mathrm{E}-02$ $1.0000000 \mathrm{E}-02$ $1.0000000 \mathrm{E}-02$ $1.0000000 \mathrm{E}-02$ $1.0000000 \mathrm{E}-02$ $1.0000000 \mathrm{E}-02$ $1.0000000 \mathrm{E}-02$ $1.0000000 \mathrm{E}-02$ $1.0000000 \mathrm{E}-02$ $.0000000 \mathrm{E}-02$ $.0000000 \mathrm{E}-02$ $.0000000 \mathrm{E}-02$ $.0000000 \mathrm{E}-02$ $1.0000000 \mathrm{E}-02$ $1.4248336 \mathrm{E}-02$ $6.6627163 \mathrm{E}-02$ $7.6120935 \mathrm{E}-02$ $1.1847159 \mathrm{E}-01$ $1.2820679 \mathrm{E}-01$ $1.2964499 \mathrm{E}-01$ $1.3075417 \mathrm{E}-01$
$1.7593470 \mathrm{E}-01$ $1.7593470 \mathrm{E}-01$
$1.9476303 \mathrm{E}-01$ $2.2670657 \mathrm{E}-01$ 
